\title{
DEVELOPMENT AND APPLICATION OF PERFECTLY MATCHED LAYER FOR NUMERICAL BOUNDARY TREATMENT
}

\author{
A Thesis by \\ Srinivasa Perumal Velu \\ Bachelor of Aeronautical Engineering, Bharathiar University, 2001
}

Submitted to the Department of Aerospace Engineering and the faculty of the Graduate School of

Wichita State University

in partial fulfillment of

the requirements for the degree of

Master of Science

May 2010 
(C) Copyright 2010 by Srinivasa Perumal Velu

All Rights Reserved

Note that thesis and dissertation work is protected by copyright, with all rights reserved. Only the author has the legal right to publish, produce, sell, or distribute this work. Author permission is needed for others to directly quote significant amounts of information in their own work or to summarize substantial amounts of information in their own work. Limited amounts of information cited, paraphrased, or summarized from the work may be used with proper citation of where to find the original work. 


\section{DEVELOPMENT AND APPLICATION OF PERFECTLY MATCHED LAYER FOR NUMERICAL BOUNDARY TREATMENT}

The following faculty members have examined the final copy of this thesis for form and content, and recommend that it be accepted in partial fulfillment of the requirement for the degree of Master of Science with a major in Aerospace Engineering

Klaus A. Hoffmann, Committee Chair

Kamran Rokhsaz, Committee Member

Ikramuddin Ahmed, Committee Member 


\section{DEDICATION}

To my family, my teachers, and my dear friends 


\section{ACKNOWLEDGEMENTS}

I would like to express profound gratitude to my advisor, Klaus A. Hoffmann, for his thoughtful, patient guidance, his unflinching encouragement, and support in various ways, without which this thesis would not have been possible. I am grateful for JeanFrançois Dietiker for providing me with resources to begin this work. I would also acknowledge Victor Isakov for his support in the area of mathematics. I also extend my gratitude to members of my committee for their helpful comments and suggestions during all stages of this work. 


\section{ABSTRACT}

This master's-level thesis work had focused on the boundary treatments for computational fluid dynamics problems, especially those with unbounded domains. Therefore, it involved a rigorous literature survey on boundary treatment techniques. The primary interest in this effort was on one of the emerging concepts of boundary treatment for numerical schemes, namely the perfectly matched layer (PML) absorbing technique. PML equations were constructed in both Cartesian and generalized coordinate systems to widen its application in uniform and non-uniform grid structures. Throughout the study, Euler equations for perturbation equations linearized about a uniform mean flow were utilized, and flow that is parallel to an axis and flow that is at an arbitrary direction were considered. The PML formulation was validated and PML cases were simulated for a combination of absorbing coefficients and layer thickness in order to find the optimum performance of the technique. The results were analyzed, and the issues were documented. Furthermore, PML equations were constructed by splitting the velocity into three components. Discussion is provided on the need for a proper spacetime transformation, based on the literature study. 


\section{TABLE OF CONTENTS}

Chapter

Page

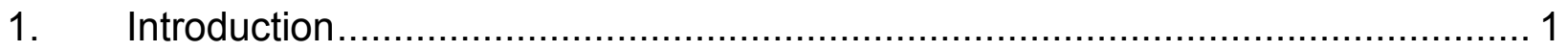

1.1. Non-Reflecting Boundary Conditions ................................................ 2

1.2. Numerical Schemes for Boundary Treatment ..................................... 3

1.2.1. Simple Extrapolation .......................................................... 3

1.2.2. Method Based on Characteristics .......................................... 4

1.2.3. Absorbing Layer/Zone Technique ........................................... 4

1.2.4. Genesis of Perfectly Matched Layer Technique........................... 5

1.2.5. Development of PML in CFD and CAA ..................................... 6

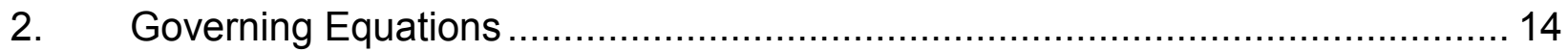

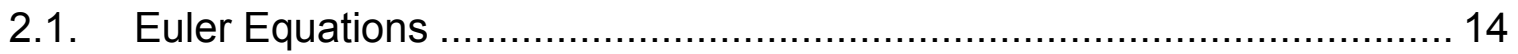

2.2. Linearized Euler Equations ............................................................ 14

2.3. Linearized Euler Equations with a Non-uniform Mean Flow................... 16

2.4. Linearized Euler Equations with a Uniform Mean Flow......................... 18

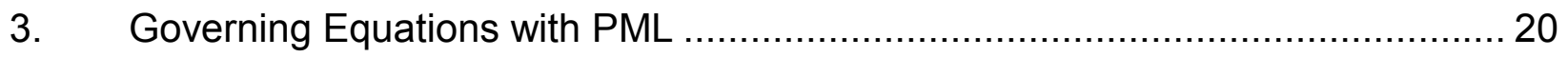

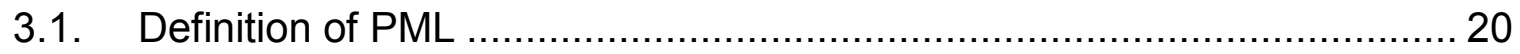

3.2. PML Equations in Cartesian Coordinates ...................................... 21

3.3. Reflection and Transmission at Interfaces between Two PMLs.............. 24

3.4. PML Equations in Generalized Coordinates ....................................... 32

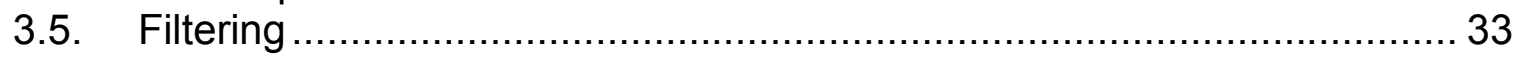

4. Numerical Scheme for Time Domain .............................................................. 34

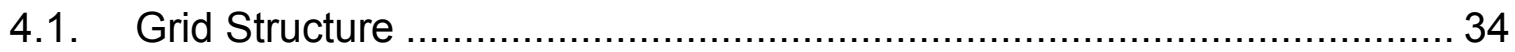

4.1.1. Spatial Discretization......................................................... 35

4.1.2. Temporal Integration ...................................................... 37

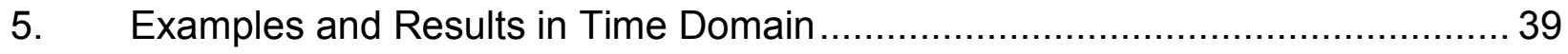

5.1. Validation of PML Formulation ..................................................... 40

5.2. PML vs. Simple Extrapolation Boundary—A Comparative Evaluation ..... 45

5.3. Validation of PML Equations in Generalized Coordinates...................... 50

5.3.1. Parallel Flow Case ........................................................... 50

5.3.2. Oblique Mean Flow Case ........................................................... 62

5.4. Investigation of PML Parameters .................................................... 72

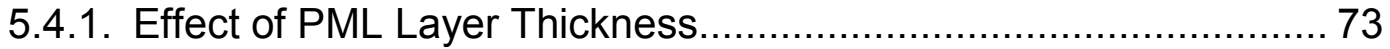

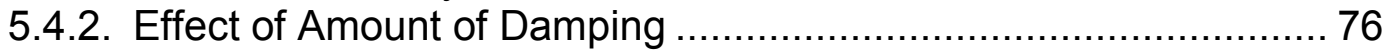




\section{TABLE OF CONTENTS (continued)}

Chapter

Page

5.4.3. Effect of Damping Rate 79

6. Difficulties Encountered in Developing PML for Oblique Mean Flow ................. 82

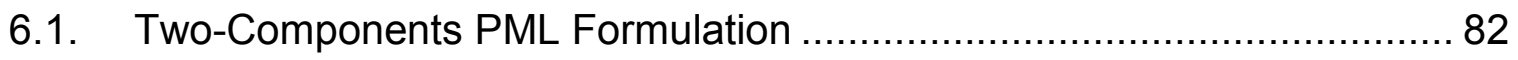

7. Development of PML Equations Using Three-Components Splitting................. 88

7.1. PML Equations Using Three-Components Splitting ............................ 88

7.2. Numerical Investigation.............................................................. 90

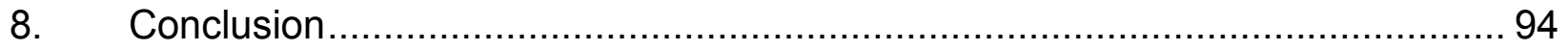

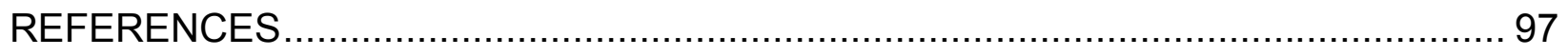

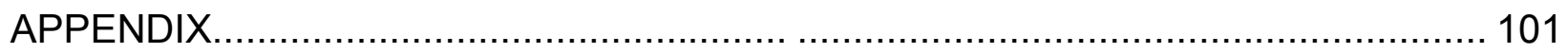

Governing Equations in Terms of Perturbation Variables...............................102 


\section{LIST OF FIGURES}

Figure

Page

3.1 Schematic of computational domain showing interior domain and PML domain on the boundary with absorption coefficients in the PML domain

3.2 A schematic showing the angles of the acoustic wave at an interface ..............25

4.1 (a) Uniform grid structure, (b) Non-uniform grid structure .............................. 35

5.1 Computational domain for uniform grid: (a) grid structure, (b) PML domain and physical space. Number of layers $=10$

5.2 Density contours at values of $\pm 0.1, \pm 0.05, \pm 0.01$, and \pm 0.005 , showing acoustic and vorticity pulses: $M_{x}=0.5$ and $M_{y}=0.0$ at $(a) t=10$, (b) $t=40$, $(c) t=50$, and $(d) t=70$, with $\sigma_{m}=\beta=2$ and NPML $=10$

5.3 Velocity $(u)$ contours at values of $\pm 0.1, \pm 0.05, \pm 0.01$, and \pm 0.02 , showing acoustic and vorticity pulses: $M_{x}=0.5$ and $M_{y}=0.0$ at $(a) t=10,(b) t=40$, (c) $t=50$, and $(d) t=70$, with $\sigma_{m}=\beta=2$ and NPML $=10$

5.4 Density along line $y=0$ for $M_{x}=0.5$ and $M_{y}=0.0$ at $(a) t=40,(b) t=50$, and $(c) t=70$, with $\sigma_{m}=\beta=2$ and NPML $=10$

5.5 Density contours at values of $\pm 0.05, \pm 0.02, \pm 0.01$, and \pm 0.008 , near the right boundary, showing the decaying of waves in the PML domain: $M_{x}=0.5$ and $M_{y}=0.0$ at (a) $t=40$, (b) $t=50$, (c) $t=60$, and $(d) t=70$, with $\sigma_{m}=\beta=2$ and NPML $=10$

5.6 Velocity $(v)$ contours at values of $\pm 0.05: M_{x}=0.9$ and $M_{y}=0.0$ at $t=50$ : (a) reference solution, (b) zero-order extrapolation, (c) PML solution with ten grid points, and (d) solution in interior domain and PML domain

5.7 Velocity $(v)$ distribution along $y=0$ line with $M_{x}=0.9$ and $M_{y}=0.0$ at $t=50$

5.8 Velocity $(v)$ component as a function of time at point $(x, y)=(50,5)$

5.9 Difference between PML and simple extrapolation solutions as a function of time at point $(x, y)=(50,5)$

5.10 Uniform grid: (a) grid structure, (b) PML domain and physical space.

Non-uniform grid: (c) grid structure, (d) PML domain and physical space.

Number of layers $=25$ 


\section{LIST OF FIGURES (continued)}

Figure

Page

5.11 Density contours at values of \pm 0.1 and \pm 0.05 with uniform and non-uniform grids, showing acoustic pulse: $M_{x}=0.5$ and $M_{y}=0.0$ at $(a) t=30$ and (b) $t=80$ .54

5.12 Density along line $x=0$ with $M_{x}=0.5$ and $M_{y}=0.0$ at $t=30$ 55

5.13 Density along line $x=-24$ with $M_{x}=0.5$ and $M_{y}=0.0$ at $t=80$

5.14 Velocity $(u)$ contours at values of \pm 0.1 and \pm 0.05 with uniform and non-uniform grids, showing acoustic pulse: $M_{x}=0.5$ and $M_{y}=0.0$ at $(a) t=30$ and (b) $t=80$

5.15 Velocity $(u)$ component along line $x=0$ with $M_{x}=0.5$ and $M_{y}=0.0$ at $t=30$

5.16 Velocity $(u)$ component along line $x=-24$ with $M_{x}=0.5$ and $M_{y}=0.0$ at $t=80$

5.17 Density contours at values of \pm 0.1 and \pm 0.05 with uniform and non-uniform grids, showing acoustic and vorticity pulses: $M_{x}=0.5$ and $M_{y}=0.0$ at (a) $t=10$ and $(b) t=40$

5.18 Density along line $x=0$ with $M_{x}=0.5$ and $M_{y}=0.0$ at $t=10 \ldots \ldots \ldots \ldots \ldots \ldots \ldots . . .59$

5.19 Density along line $x=0$ with $M_{x}=0.5$ and $M_{y}=0.0$ at $t=40$

5.20 Velocity $(u)$ contours at values of \pm 0.1 and \pm 0.05 with uniform and non-uniform grids, showing acoustic and vorticity pulses: $M_{x}=0.5$ and $M_{y}=0.0$ at

(a) $t=40$ and $(b) t=70$

5.21 Velocity $(u)$ component along line $x=-20$ with $M_{x}=0.5$ and $M_{y}=0.0$ at $t=40$

5.22 Velocity $(u)$ component along line $x=-15$ with $M_{x}=0.5$ and $M_{y}=0.0 a t$ $t=70$

5.23 Density contours at values of \pm 0.1 and \pm 0.05 with uniform and non-uniform grids, showing acoustic pulse: $M_{x}=0.5 \sin (\pi / 4)$ and $M_{y}=0.5 \sin (\pi / 4)$ at (a) $t=30$ at (b) $t=80$ 


\section{LIST OF FIGURES (continued)}

Figure

Page

5.24 Density along line $x=0$ with $M_{x}=0.5 \sin (\pi / 4)$ and $M_{y}=0.5 \sin (\pi / 4)$ at $t=30$

5.25 Density along line $x=0$ with $M_{x}=0.5 \sin (\pi / 4)$ and $M_{y}=0.5 \sin (\pi / 4)$ at $t=80$

5.26 Velocity $(u)$ contours at values of \pm 0.1 and \pm 0.05 with uniform and non-uniform grids, showing acoustic pulse: $M_{x}=0.5 \sin (\pi / 4)$ and $M_{y}=0.5 \sin (\pi / 4)$ at (a) $t=30$ and (b) $t=70$

5.27 Velocity $(u)$ component along line $x=0$ with $M_{x}=0.5 \sin (\pi / 4)$ and $M_{y}=0.5 \sin (\pi / 4)$ at $t=30$

5.28 Velocity $(u)$ component along line $x=0$ with $M_{x}=0.5 \sin (\pi / 4)$ and $M_{y}=0.5 \sin (\pi / 4)$ at $t=70$

5.29 Density contours at values of \pm 0.1 and \pm 0.05 with uniform and non-uniform grids, showing acoustic and vorticity pulses: $M_{x}=0.5 \sin (\pi / 4)$ and $M_{y}=0.5 \sin (\pi / 4)$ at $(a) t=10$ and $(b) t=40$

5.30 Density along line $x=0$ with $M_{x}=0.5 \sin (\pi / 4)$ and $M_{y}=0.5 \sin (\pi / 4)$ at $t=10$

5.31 Density along line $x=0$ with $M_{x}=0.5 \sin (\pi / 4)$ and $M_{y}=0.5 \sin (\pi / 4)$ at $t=40$

5.32 Velocity $(u)$ contours at values of \pm 0.05 with uniform and non-uniform grids, showing acoustic and vorticity pulses: $M_{x}=0.5 \sin (\pi / 4)$ and $M_{y}=0.5 \sin (\pi / 4)$ at $(a) t=40$ and $(b) t=70$

5.33 Velocity $(u)$ component along line $x=-15$ with $M_{x}=0.5 \sin (\pi / 4)$ and $M_{y}=0.5 \sin (\pi / 4)$ at $t=40$

5.34 Velocity $(u)$ component along line $x=-20$ with $M_{x}=0.5 \sin (\pi / 4)$ and $M_{y}=0.5 \sin (\pi / 4)$ at $t=70$

5.35 Pressure contours at values of \pm 0.001 for various PML thicknesses,

$\sigma_{\max }=2.0$, and $\beta=2.0: M_{x}=0.5$ and $M_{y}=0.0$ at $t=90$ 74 


\section{LIST OF FIGURES (continued)}

Figure

Page

5.36 Pressure along $y=0$ line with PMLs of various thicknesses,

$\sigma_{\max }=2.0$, and $\beta=2.0: M_{x}=0.5$ and $M_{y}=0.0$ at $(a) t=50$ and

(b) $t=100$

5.37 Pressure contours at values of \pm 0.001 for various $\sigma_{\max }$ and $\beta=2.0$ :

$M_{x}=0.5$ and $M_{y}=0.0$ at $t=100$, with NPML $=25$

5.38 Pressure along $y=0$ line with PMLs of various $\sigma_{\max }$ and $\beta=2.0$ :

$M_{x}=0.5$ and $M_{y}=0.0$ at $(a) t=50$ and $(b) t=100$, with NPML $=25$

5.39 Pressure contours at values of \pm 0.005 for various $\beta$ and $\sigma_{\max }=2.0$ :

$M_{x}=0.5$ and $M_{y}=0.0$ at $t=50$, with NPML $=25$

5.40 Pressure along $y=0$ line with PMLs of various $\beta$ and $\sigma_{\max }=2.0$ :

$M_{x}=0.5$ and $M_{y}=0.0$ at $(a) t=50$ and $(b) t=100$, with NPML $=25$

6.1 Density contours at values of $\pm 0.5, \pm 0.05, \pm 0.01$, and \pm 0.005 , showing acoustic and vorticity pulses: $M_{x}=0.5$ and $M_{y}=0.0$ at $(a) t=0,(b) t=70$,

(c) $t=100$, and $(d) t=200$, with NPML $=10, \sigma_{\max }=2.0$, and $\beta=3.0$

6.2 Density contours at values of $\pm 0.5, \pm 0.05, \pm 0.01$, and \pm 0.005 , showing acoustic and vorticity pulses: $M_{x}=0.0$ and $M_{y}=0.5$ at $(a) t=0,(b) t=70$, (c) $t=100$, and $(d) t=200$, with NPML $=10, \sigma_{\max }=2.0$, and $\beta=3.0$

6.3 Density contours at values of \pm 0.5 and \pm 0.1 , showing acoustic and vorticity pulses: $M_{x}=0.5$ and $M_{y}=0.5$ at $(a) t=0,(b) t=70,(c) t=100$, and (d) $t=200$, with NPML $=10, \sigma_{\max }=2.0$, and $\beta=3.0$

7.1 Density contours at values of \pm 0.1 , showing acoustic and vorticity pulses for $M_{x}=0.5$ and $M_{y}=0.5$ at $(a) t=70$ and $(b) t=100$, with NPML $=10$, $\sigma_{\max }=2.0$, and $\beta=3.0$ 


\section{LIST OF ABBREVIATIONS/NOMENCLATURE}

Roman Scalar Variables

a

CAA

CFD

D

d

DNS

$e_{i}$

$e_{t}$

$\mathrm{H}$

$h$

$i$

$J$

$\mathrm{K}$

$l$

$M$

NPML

NRBC

ODE

$p$

PDE

PML
Speed of sound

Computational aero acoustics

Computational fluid dynamics

Thickness of PML

Distance of a point in PML from the interface

Direct numerical solution

Internal energy

Total energy

Damping term

Enthalpy

Complex notation representing $\sqrt{-1}$

Determinant of Jacobian of transformation

Wave number

Length

Mach number

Number of layers in PML domain

Non-reflecting boundary condition

Ordinary differential equation

Pressure

Partial differential equation

Perfectly matched layer 


\section{LIST OF ABBREVIATIONS/NOMENCLATURE (continued)}

$\begin{array}{ll}\text { Q } & \text { Flux vector } \\ t & \text { Time } \\ u & \text { X component of velocity } \\ v & \text { Y component of velocity } \\ w & \text { Z component of velocity } \\ x & \text { X coordinate } \\ y & \text { Y coordinate } \\ z & \text { Z coordinate }\end{array}$

Vector and Matrix Variables

E

F

$Q$

$H$

Greek Scalar Variables

$\alpha$

$\beta$

$\beta$

$\rho$

$\sigma$

$\mu$

$\Phi$

$\omega$
Spatial flux vector in $x$ or $\xi$ direction

Spatial flux vector in $y$ or $\eta$ direction

Vector of dependent variables

Vector of absorption terms
Angle of inclination of mean flow with $x$ axis

Damping distribution rate

Parameter in transformation used by $\mathrm{Hu}$

Density

Amount of damping

Parameter in the PML construction of Tam

Angle made by the wave with respect to $x$ axis

Wave frequency 


\title{
LIST OF ABBREVIATIONS/NOMENCLATURE (continued)
}

$\xi$

$\eta$

Superscripts and Subscripts

()$^{*}$

()$^{\Delta}$

()$^{\prime}$

$\overline{()}$

()$_{i}$

()$_{r}$

()$_{t}$

()$_{0}$
Independent variable in computational space

Independent variable in computational space

\author{
Non-dimensionalized parameter \\ Difference between actual and mean flow quantity \\ Perturbation quantity \\ Steady mean flow quantity \\ Incident quantity \\ Reflected quantity \\ Transmitted quantity \\ Free stream quantity
}




\section{CHAPTER 1}

\section{INTRODUCTION}

Fluid dynamics, the science of fluid motion, is commonly studied in one of three approaches viz., experimental fluid dynamics, theoretical fluid dynamics and computational fluid dynamics (CFD). Thus, CFD is the science of predicting fluid flow, heat transfer, chemical reactions, and related phenomena by solving the system of partial differential equations (PDE) which governs the fluid motion or wave propagation using numerical schemes. The potential of CFD techniques has evolved into many dimensions in the past few decades, with the tremendous technological growth in areas of numerical computing. To obtain unique and accurate solution for a system of PDEs, one must specify appropriate initial and boundary conditions for the problem at hand, which is a challenging and vital issue, since the imposed conditions have serious effects on the computed solution. Therefore, the treatment of boundary conditions has become very crucial and of paramount importance in order to achieve time-accurate solutions. In some applications, these boundary conditions are known from the physics of the problem, but in most cases, they are not known a priori, since they depend both on the interior solution as well as information coming from the exterior domain of the problem [1].

Fluid dynamics problems can be either a closed domain, internal flow problem or an open domain, external flow problem. It is relatively simple to define the boundary conditions for a closed domain problem, since the boundaries for these problems are well defined, and the behavior of the physical properties on these boundaries is well understood. But the case is highly complex when the spatial domain of the problem at 
hand is unbounded. In that situation, to make a finite computational domain, a numerical treatment usually requires the introduction of an artificial boundary, and the imposed boundary conditions on these mimicked boundaries are solely responsible for the correct representation of the truncated domain and the physical phenomena that occur therein [2]. If these artificial boundaries are not transparent to out-going disturbances, then the reflection of large spurious waves from these artificial boundaries contaminates the flow field and eventually may lead to large errors and spoil the numerical solution. A detailed discussion on spurious waves and the artifacts of numerical discretization can be found in the review on computational aeroacoustics (CAA) by Lele [3].

\section{$1.1 \quad$ Non-Reflecting Boundary Conditions}

The literature is abundant with a variety of boundary treatments for these problems, and in general, they are termed non-reflecting boundary conditions (NRBCs). Givoli [2] carried out a detailed review of the application of NRBC in various fields of science, the methodologies used by different authors, the advantages and disadvantages of the numerical schemes, and the challenges ahead. PDEs that govern the fluid flow can be approximated by numerous numerical schemes, and in the same way, several numerical approximations have been developed to approximate the associated boundary conditions. Thus, for a given problem, there is an abundance of numerical schemes, which vary widely in degree of sophistication, accuracy and implementation. Hoffmann and Chang [1] grouped the schemes into three major categories: (1) schemes where the boundary conditions are specified by simple extrapolation; (2) schemes that are based on the theory of characteristics, where a set of equations are solved to provide the required unknown data on the boundary; and (3) 
schemes that involve absorbing layers or sponge layers in which additional zones of grid points, or layers, surround the artificial boundary to diminish the strength of the outgoing disturbances before they reach the boundary using artificial dissipation or damping in order to prevent reflection from the boundary. Loh and Jorgenson [4] grouped categories (1) and (2) together and termed them "hard absorbing NRBCs" and termed category (3) as "soft absorbing NRBCs".

Givoli [2] suggested that one should ensure the following when devising a new NRBC: (1) a mathematically well-posed problem in the domain with the boundary condition, (2) good approximation of the original problem, (3) a highly compatible boundary condition with the numerical solution, (4) stable numerical scheme, (5) a small spurious reflection from the boundary, (6) reduced computational effort, and (7) a faster approach to steady-state condition.

\subsection{Numerical Schemes for Boundary Treatment}

\subsubsection{Simple Extrapolation}

Simple extrapolation schemes have strict stability requirements to be met and require a large computational domain for better results, which will eventually increase the computational time and cost of analysis. On the other hand, if a small domain is used in order to reduce the computational effort, waves from the boundaries reflect back into the numerical domain and contaminate the solution. This case turns out to be worse when acoustic waves are reflected back into the solution. Since acoustic waves travel at the speed of sound, the spurious reflections come back into the solution and destroy the numerical solution in a short period of time. This is demonstrated in section 5.2. 


\subsubsection{Method Based on Characteristics}

The NRBC based on characteristics is a popular numerical treatment, but it has complicated procedures. This approach is based on the local one-dimensional characteristic decomposition of the flow variables [3]. A full set of characteristics can be easily found for one-dimensional Euler equations. However, there are no true characteristics in two or three dimensional problems. They work well when the incident acoustic waves are normal to the boundary. However, they do not provide good results in the presence of a strong, mean flow that is tangent to the boundary [5]. Furthermore, depending on the problem, different forms of equations are required. Therefore, a set of general equations based on the characteristics are derived and subsequently modified as per the boundary requirements [1]. Regardless of its wide application based on the characteristics of the linearized equations and their asymptotic solutions in the far-field boundary treatments, they become unsatisfactory when the outflow is non-linear or involves multi-directional waves [6].

\subsubsection{Absorbing Layer/Zone Technique}

Several research activities have involved the soft-absorbing NRBC, and it has been shown that this boundary condition helps achieve simulations in truncated domains that are much smaller than the physical space where the phenomena takes place. This is obtained by creating a buffer zone abutting the computational boundary. The governing equations are modified in order to remove or dampen the reflected waves that are oriented back towards the computational domain, i.e., to absorb the incident waves. In other words, the buffer zone is constructed in such a way that the outgoing waves/disturbances are progressively attenuated before they encounter the 
computational boundary. It must be understood that the solutions in the buffer zone need not necessarily be physical. The main purpose of this zone is to prevent contamination of the solution in the physical domain of interest by reflections from the computational boundaries.

It is well known that the Euler equations, due to their hyperbolic nature, support linear continuous waves, viz., pressure pulses, entropy and vorticity waves, and nonlinear or discontinuous waves such as shock waves. Hence, it becomes inevitable to understand the theory involved in the development of PML at the preliminary level and its application in CAA, which is one of the major areas of research in computational physics. Tam [5] reviews, in detail, the advances in numerical boundary conditions for CAA. He reiterates the importance of treating the inflow and outflow radiation boundary conditions differently, owing to the nature of acoustic waves being reflected back into the solution, i.e., acoustic waves are the only outgoing waves at the inflow boundary. On the other hand, outgoing waves at the outflow boundary are a combination of acoustic, vorticity, and entropy waves. It must be remembered that the acoustic waves travel at the speed of sound, whereas vorticity and entropy waves are frozen patterns that are convected downstream by the mean flow. Nizampatnam et al. $[7,8]$ carried out extensive investigation work on boundary conditions for computational aeroacoustics

and development of PML for aeroacoustic applications in the time domain and the frequency domain.

\subsubsection{Genesis of Perfectly Matched Layer Technique}

One of the greatest challenges in electromagnetics has been to solve the Maxwell equations in free-space simulation, where the governing equations had to be 
solved in a discretized domain with restrained sizes, and the existing techniques of that time imposed too many constraints and caused difficulties when artificial boundaries were created. Berenger made a breakthrough in 1994 [9] by proposing a new technique based on the use of an absorbing layer to solve unbounded electromagnetic problems with the finite difference time domain method. In this method, the computational domain was truncated by a layer that attenuates the outgoing electromagnetic waves of the Maxwell equations, regardless of their frequency and angle of incidence, i.e., the governing equations of the absorbing layer are matched with the equations of the computational domain in such a way that there exists no reflection at the interface. Berenger split the governing equations of the matched layer into subcomponents in an unphysical manner and introduced additional degrees of freedom, which allowed him to construct the absorbing layer with damping terms that absorb the incident waves almost perfectly.

\subsubsection{Development of PML in CFD and CAA}

Inspired by the success of the PML method for Maxwell's equations, Hu constructed the first PML equations for aeroacoustic problems [10]. He applied Berenger's PML technique to the governing Euler equations linearized over a uniform mean flow. Following Berenger's original formulation for the Maxwell equations, he split the dependent variables, i.e., velocity, pressure, and density, in two- space dimensions in the PML domain and constructed the PML formulation for both linear and non-linear Euler equations [11]. One important fact is that Berenger split only some of the field components, whereas Hu split all dependent variables to obtain the formulation in the absorbing layer. The introduction of absorption terms imposed additional stability 
requirements, and he chose a tenth-order numerical filter applied every ten time steps to reduce the amplitude of short waves, since his choice of central differencing resolved only a limited range of long waves and did not resolve the short waves [11]. Subsequently, he extended his work to uniform and non-uniform mean flow for linear as well as non-linear Euler and Navier-Stokes equations [12-21]. He also applied his formulation of PML for wave mode analysis in ducted environments. Following $\mathrm{Hu}$, Goodrich and Hagstrom [22], in their formulation, assumed the $y$-component of velocity to be zero, imposed damping only in the $x$ direction and obtained a formulation without any filter, which was different from that of $\mathrm{Hu}$ [10]. In fact, it was not clear to them for what reasons their solution failed when the simulation was carried out for a longer period of time. They attributed the reason for the constructed PML problem not being well-posed to this failure under the conditions of their experiments in a more general context.

Later, Abarbanel and Gottlieb [23], in their analysis of electromagnetic PML equations, showed that the Berenger's split-form PML formulation was only weakly wellposed. They conducted a detailed mathematical analysis of Berenger's PML formulation and investigated the initial value problem. It was mathematically shown that the split form of PML has lost the symmetry and, unlike the original Maxwell's equations, was not strongly well-posed, thus resulting in a diverging solution under small perturbations. It was unfortunate that the technique proposed by Berenger led to a system that lost the most important properties of the original one: strong hyperbolicity and symmetry [24].

This was verified by Hesthaven [25] through his similarity transformation analysis for both original un-split equations and the split-form PML equations proposed by 
Goodrich and Hagstrom [22] and Hu [10]. From his analysis, the application of PML for acoustic problems was validated and shown that the formulation is well suited for numerical solution, but the weakly well-posed systems may become ill-posed under loworder perturbations, thus leaving a system with intrinsic instability. This instability was overcome by $\mathrm{Hu}$ [11] using a filtering technique. However, in reality, it is not easy to know a priori when one should apply filtering. Further, by combining different techniques, Hesthaven developed a formulation that had a PML like behavior. This scheme requires a greater number of grid points in order to obtain the solution at acceptable levels.

Despite these issues, the development of PML for acoustic problems was a sensation in the 1990s and continues to be. Tam and Auriault [26] extended the stability analysis through the dispersion relations of the PML equations and considered the role of phase signs and group velocities. A detailed discussion of the artifacts of numerical discretization is given by Lele [3]. The signs of group velocities of long waves and spurious/short waves play a key role in the numerical solution. When these velocities have opposite signs in a mean flow case, the transmitted waves, instead of being damped in the PML, will actually grow spatially, thus leading to a diverging solution. This issue is irrelevant when there is no mean flow and all the solutions are stable, however, when the mean flow is not zero, PML equations in the presence of a uniform flow support an unstable solution and grow spatially. Tam and Auriault used artificial damping in order to obtain a stable solution. This is accomplished by absorbing only the short waves, which does not adversely affect the long waves. 
Subsequently, the idea of conserving the dispersion relation of the physical space equations was explored. Now, Abarbanel et al. [27] continued to apply their PML technique for electromagnetics to the advective aeroacoustic problem. Before constructing the PML equations, they used a coordinate transformation in such a way that the transformed coordinates preserved the dispersion relation. From Abarbanel and Gottlieb's work on the well-posedness issue [23], the authors constructed a PML without splitting the physical variables. Despite its proven well-posedness, their formulation has exponential growth of reflections due to one of their eigenvalue solutions having a positive value. This is a major problem when long time integration is required. The authors refer this problem to a Jordan block structure in the system of ordinary differential equations (ODE). To overcome this problem, they suggested shifting the positive eigenvalues into the negative half plane and breaking the Jordan block by splitting the multiple eigenvalue. Their method can be applied in a situation where a rotation is used to align the mean flow with the computational grid. However, when the mean flow is not aligned to one of the axes, which is the case in general situations, an appropriate transformation will be required, and there exists no such transformation. Hence, the formulation has restricted flow directions. Determining a special transformation to overcome this issue has been a challenge for many years. Investigators in PML problems continue to research and develop a special transformation in which the waves are absorbed for all wave numbers, thus resulting in a reflection-free PML formulation that is well-posed.

Following the concept of Abarbanel et al. [27], Hu [12] applied a complex change of variables for $x$ and $y$, where the governing equations are expressed in frequency 
domain. Finally, he obtained the unsplit formulation in the time domain by introducing an auxiliary variable. He attributed the exponential growth of the reflections to the angle $\phi$, the angle of the wave-front normal vector that appears in the plane wave solution given by equation (3.31). In wave propagation, a wave is right going or left going when the group velocity is positive or negative, respectively. However, the group velocity is not always in the same direction with that of the phase velocity and this will lead to exponential growth of the waves when they enter the PML domain, instead of being suppressed, thus leading to instability. To obtain a stable PML, he used a transformation in order to align the group velocities and the phase velocities in the same direction. He continues to extend his un-split formulation for three-dimensional cases but this time with absorption coefficients linearly changing in the PML domain [13].

Hagstrom and Nazarov have extended the PML formulation for jet flow simulations [28] and shear flow calculations [29], which is far more challenging than the problem of electromagnetics. This is because even small disturbances in the boundary will affect the medium of interest as it is layered due to the shear, and the instabilities may cause large vortices. They developed the PML for both uniform and non-uniform flow by performing a Fourier-Laplace transformation that introduces additional parameters, $r$ and $\mu$, which they termed as localizable pseudo-differential operators. For the uniform flow, they could calculate a constant value for $\mu$ from the eigenvalues for which all waves can be damped. However, in the case of non-uniform flow, $\mu$ could not be calculated and the solution resulted in high frequency instabilities for poor values of $\mu$. The modified PML of Hagstrom and Nazarov [29] included up to three additional parameters. The cumbersome task of adjusting these parameters and the associated 
sensitivity of these parameters on the accuracy of the solution is a significant defect of the formulation. The parameter $\mu$ used by Hagstrom and Nazarov is similar to the parameter $\beta$ used by Hu [14].

$\mathrm{Hu}$ [14] in his formulation of PML for non-uniform flow, used the spectral collocation method. This scheme provides a general way to determine the dispersion relation for arbitrary mean flow to solve the eigenvalue problem. This is performed in order to obtain the value of $\beta$ that appears in the space-time transformation. $\mathrm{Hu}$ determined that the PML equations support unstable modes when the absorption coefficient exceeds a limiting value, where the limiting value varies with the mean flow profile used in the analysis. He suggested grid stretching or introducing the advection term to the equations to overcome this limitation. He further demonstrated that the formulation could produce good results with the application of numerical filtering and grid stretching. Parrish and $\mathrm{Hu}$ [19] encountered a large amount of reflections at the corners, rather than at the sides, when they simulated an oblique mean flow case with three vorticity pulses and one acoustic pulse. Although there is a considerable amount of error, the increased layer thickness reduced the error (in order of $10^{-2}$ ) and this error remains the same at large time levels, i.e., there is no growth in the error at the final solution. The conditions used by Parrish and Hu [19] have been employed in the current work and the results are discussed in Chapter 6. From his success on the linear Euler equations, $\mathrm{Hu}$ extended his formulation for non-linear Euler and Navier-Stokes equations [20], where he used the concept of pseudo mean flow to absorb the nonlinear disturbances. 
Although numerous authors in different fields have contributed to the research of PML, the work of $\mathrm{Hu}$ in developing the PML for CFD and CAA should be well appreciated. Since the time he realized the importance of space-time transformation to obtain a reflection-free PML formulation, he followed three major steps in deriving the PML formulation. First, he applied a space-time transformation to the governing equations. To reiterate, this first step is the most critical one because a space-time transformation must be determined in such a way that, in the transformed coordinates, all the waves supported by the governing equations will have consistent phase and group velocities, and thus all the waves will be absorbed for any wave number. Details can be found in the work of Lele [3] and Hu [12]. This is accomplished by analyzing the dispersive waves of the Euler equations, i.e., the dispersion relations for the waves supported by the governing equations are arrived at and provide further assurance that these relations are stable, which in turn stabilizes the PML construction. In the second step, Hu applied a PML complex change of variables in the frequency domain, and in the third step, he obtained the time-domain absorbing boundary condition by a conversion of the frequency-domain equations. This approach has worked well. However, the difficulty is the analysis of the dispersive waves and obtaining an appropriate space-time transformation. There exists no generalized transformation leading to a PML formulation that can produce a reflection-free solution. Despite the difficulties associated with PML formulation in fluid-flow and aeroacoustic problems, investigators contemplate the PML technique as a potential absorbing boundary treatment where a tremendous amount of work needs to be conducted so that the PML technique can be applied efficiently to a wide variety of problems. 
Up to the present time, research activities have shown that the PML technique is a powerful tool. The application of this scheme will greatly reduce the computational cost, since a small computational domain will suffice to solve the numerical problem. In the present work, a generalized transformation was implemented. The PML equations for linearized Euler equations with a uniform mean flow were constructed. As mentioned in section 1.2.3, it has become necessary to understand the development of PML technique before it can be utilized in complicated fluid-flow problems, i.e., flow problems that involve shocks. An example of a problem involving a shock wave is given by Dietiker and Hoffmann [30] where sponge-layer technique was utilized. The objectives of the current research is to establish a platform with an understanding of the physics involved in PML, its development process, problems encountered in its development, and the alternatives. These individual objectives will be discussed in subsequent chapters. 


\section{CHAPTER 2}

\section{GOVERNING EQUATIONS}

\section{$2.1 \quad$ Euler Equations}

The two-dimensional Euler equations, in Cartesian coordinates, written in the conservative form is [1]

$$
\frac{\partial}{\partial t} Q+\frac{\partial}{\partial x} F+\frac{\partial}{\partial y} G=0
$$

The unknown vector $Q$ and the flux vectors are

$$
Q=\left[\begin{array}{c}
\rho \\
\rho u \\
\rho v \\
\rho e_{t}
\end{array}\right] ; F=\left[\begin{array}{c}
\rho u \\
\rho u^{2}+p \\
\rho u v \\
\left(\rho e_{t}+p\right) u
\end{array}\right] ; G=\left[\begin{array}{c}
\rho v \\
\rho v u \\
\rho v^{2}+p \\
\left(\rho e_{t}+p\right) v
\end{array}\right]
$$

where $\rho$ and $p$ are the density and pressure, respectively; $u$ and $v$ are the $x$ and $y$ components of velocity, respectively; $e_{t}$ and $h$ are the total energy and enthalpy of the fluid, respectively; and, for a perfect gas, $e_{t}$ and $h$ are defined as

$$
\begin{gathered}
e_{t}=e_{i}+\frac{1}{2}\left(u^{2}+v^{2}\right) \\
h=e_{i}+\frac{p}{\rho}
\end{gathered}
$$

Where $e_{i}$ is the internal energy.

\subsection{Linearized Euler Equations}

By assuming that acoustic values are a small, linear, unsteady perturbation upon a steady mean flow, the governing equations, i.e., equations (2.24) to (2.27) are derived 
directly from the Euler equations. The flow variables can be written as the sum of a mean quantity, represented by " - ", and a perturbation quantity, represented by superscript " $"$ " as follows:

$$
\begin{aligned}
u(x, y, t) & =\bar{u}(x, y)+u^{\prime}(x, y, t) \\
v(x, y, t) & =\bar{v}(x, y)+v^{\prime}(x, y, t) \\
\rho(x, y, t) & =\bar{\rho}(x, y)+\rho^{\prime}(x, y, t) \\
p(x, y, t) & =\bar{p}(x, y)+p^{\prime}(x, y, t)
\end{aligned}
$$

The governing linearized Euler equations for the perturbation acoustic quantities are derived by substituting the perturbation relations, equations $(2.5)$ to $(2.8)$, in the Euler equation given by equation (2.1). The equation for the steady mean flow and the equation for the first-order unsteady perturbation quantities are obtained by collecting terms of equal order and neglecting terms of higher order.

The dependent variables of the unsteady Euler equation, steady mean flow equations, and the perturbation variables are non-dimensionalized according to the free stream conditions, denoted by the subscript " $\infty$," as shown in equation (2.9),

$$
\begin{gathered}
\rho^{*}=\frac{\rho}{\bar{\rho}_{0}} ; \bar{\rho}^{*}=\frac{\bar{\rho}}{\bar{\rho}_{0}} ; u^{*}=\frac{u}{a_{0}} ; \bar{u}^{*}=\frac{\bar{u}}{a_{0}} ; v^{*}=\frac{v}{a_{0}} ; \bar{v}^{*}=\frac{\bar{v}}{a_{0}} ; \\
t^{*}=\frac{t a_{0}}{L} ; p^{*}=\frac{p}{\bar{\rho}_{0} a_{0}^{2}} ; \bar{p}^{*}=\frac{\bar{p}}{\bar{\rho}_{0} a_{0}^{2}}
\end{gathered}
$$

and the independent length is non-dimensionalized as shown in equation (2.10)

$$
\left(x^{*}, y^{*}\right)=\frac{(x, y)}{L}
$$

where the superscript " * " refers to non-dimensionalized quantity, a refers to speed of sound, and $L$ refers to a reference length. For the remainder of this thesis, the non- 
dimensionalized quantities will be used in the equations, and the notation " * " will be dropped hereafter.

\subsection{Linearized Euler Equations with a Non-uniform Mean Flow}

The two-dimensional, compressible Euler equations are linearized in terms of the acoustic variables about a mean flow to obtain the acoustics equations. When the mean flow is non-uniform, the governing equations for perturbation quantities contain more than one unknown mean flow quantity. In the present work, the emphasis is on the solution of perturbation equations. It is assumed that the mean flow quantities are known, computed elsewhere [8].

The steady mean flow equations are,

$$
\frac{\partial}{\partial x} \bar{F}+\frac{\partial}{\partial y} \bar{G}=0
$$

where

$$
\bar{F}=\left[\begin{array}{c}
\overline{\rho u} \\
\bar{\rho}-\bar{u}+\bar{p} \\
\overline{\rho u v} \\
\left(\bar{\rho}-\overline{e_{t}}+\bar{p}\right) \bar{u}
\end{array}\right] ; \bar{G}=\left[\begin{array}{c}
\overline{\rho v} \\
\overline{\rho v u} \\
\overline{\rho v}{ }^{2}+\bar{p} \\
\left(\overline{\rho e_{t}}+\bar{p}\right) \bar{v}
\end{array}\right]
$$

This is identical to the original equation, i.e., equation (2.1), with the time derivative term set to zero. It must be noted that the mean flow is represented by a set of coupled non-linear equations. The non-dimensionalized perturbation equations are given by equations (2.13) to (2.16). The details of the derivations are presented in Appendix.

$$
\frac{\partial u^{\prime}}{\partial t}+\bar{u} \frac{\partial u^{\prime}}{\partial x^{*}}+\bar{v} \frac{\partial u^{\prime}}{\partial y^{*}}+\frac{1}{\bar{\rho}} \frac{\partial p^{\prime}}{\partial x}=-u^{\prime} \frac{\partial \bar{u}}{\partial x}-v^{\prime} \frac{\partial \bar{u}}{\partial y}+\frac{\partial \bar{p}}{\partial x} \frac{\rho^{\prime}}{\rho^{2}}
$$




$$
\begin{gathered}
\frac{\partial v^{\prime}}{\partial t}+\bar{u} \frac{\partial v^{\prime}}{\partial x}+\bar{v} \frac{\partial v^{\prime}}{\partial y}+\frac{1}{\bar{\rho}} \frac{\partial p^{\prime}}{\partial y}=-u^{\prime} \frac{\partial \bar{v}}{\partial x}-v^{\prime} \frac{\partial \bar{v}}{\partial y}+\frac{\partial \bar{p}}{\partial y} \frac{\rho^{\prime}}{\bar{\rho}^{2}} \\
\frac{\partial p^{\prime}}{\partial t}+\bar{u} \frac{\partial p^{\prime}}{\partial x}+\bar{v} \frac{\partial p^{\prime}}{\partial y}+\gamma \bar{p}\left(\frac{\partial u^{\prime}}{\partial x}+\frac{\partial v^{\prime}}{\partial y}\right)=-u^{\prime} \frac{\partial \bar{p}}{\partial x}-v^{\prime} \frac{\partial \bar{p}}{\partial y}-\gamma\left(\frac{\partial \bar{u}}{\partial x}+\frac{\partial \bar{v}}{\partial y}\right) p^{\prime} \\
\frac{\partial \rho^{\prime}}{\partial t}+\bar{u} \frac{\partial \rho^{\prime}}{\partial x}+\bar{v} \frac{\partial \rho^{\prime}}{\partial y}+\bar{\rho}\left(\frac{\partial u^{\prime}}{\partial x}+\frac{\partial v^{\prime}}{\partial y}\right)=-\rho^{\prime}\left(\frac{\partial \bar{u}}{\partial x}+\frac{\partial \bar{v}}{\partial y}\right)-u^{\prime} \frac{\partial \bar{\rho}}{\partial x}-v^{\prime} \frac{\partial \bar{\rho}}{\partial y}
\end{gathered}
$$

In conservative form, the first-order perturbation equations are given by

$$
\frac{\partial}{\partial t} Q+\frac{\partial}{\partial x} F+\frac{\partial}{\partial y} G=0
$$

where

$$
Q=\left[\begin{array}{c}
\rho^{\prime} \\
(\rho u)^{\Delta} \\
(\rho v)^{\Delta} \\
(\rho e)^{\Delta}
\end{array}\right] ; F=\left[\begin{array}{c}
(\rho u)^{\Delta} \\
\bar{u}\left[2(\rho u)^{\Delta}-\bar{u} \rho^{\prime}\right]+p^{\prime} \\
\bar{v}\left[(\rho u)^{\Delta}-\bar{u} \rho^{\prime}\right]+(\rho v)^{\Delta} \bar{u} \\
\bar{u}\left[(\rho h)^{\Delta}-\bar{h} \rho^{\prime}\right]+(\rho u)^{\Delta} \bar{h}
\end{array}\right] ; G=\left[\begin{array}{c}
(\rho v)^{\Delta} \\
\bar{u}\left[(\rho v)^{\Delta}-\bar{v} \rho^{\prime}\right]+(\rho u)^{\Delta} \bar{v} \\
\bar{v}\left[2(\rho v)^{\Delta}-\bar{v} \rho^{\prime}\right]+p^{\prime} \\
\bar{u}\left[(\rho h)^{\Delta}-\bar{h} \rho^{\prime}\right]+(\rho v)^{\Delta} \bar{h}
\end{array}\right]
$$

The equation of state is given as

$$
p^{\prime}=(\gamma-1)\left\{(\rho e)^{\Delta}-\frac{1}{2}\left[\bar{u}\left(2(\rho u)^{\Delta}-\bar{u} \rho^{\prime}\right)+\bar{v}\left(2(\rho v)^{\Delta}-\bar{v} \rho^{\prime}\right)\right]\right\}
$$

Where $\gamma$ is the ratio of specific heats and is assumed to be 1.4 , and the " $\Delta$ " quantities are defined as

$$
\begin{gathered}
(\rho u)^{\Delta}=\rho u-\bar{\rho} \bar{u}=\bar{\rho} u^{\prime}+\rho^{\prime} \bar{u} \\
(\rho v)^{\Delta}=\rho v-\bar{\rho} \bar{v}=\bar{\rho} v^{\prime}+\rho^{\prime} \bar{v} \\
\left(\rho e_{t}\right)^{\Delta}=\rho e_{t}-\bar{\rho} \bar{e}_{t}=\frac{p^{\prime}}{\gamma-1}+\bar{\rho} \bar{u} u^{\prime}+\bar{\rho}-\bar{v} v^{\prime}+\frac{1}{2} \rho^{\prime}\left(\bar{u}^{2}+\bar{v}^{2}\right) \\
(\rho h)^{\Delta}=\rho h-\bar{\rho} \bar{h}=(\rho e)^{\Delta}+p^{\prime}
\end{gathered}
$$


These equations are linear in the perturbation values, and the mean flow values are known from the solution of equation (2.11).

\subsection{Linearized Euler Equations with a Uniform Mean Flow}

The linearized Euler equations for a uniform mean flow in the non-dimensional form is given by equations (2.24) to (2.27). The derivations are presented in Appendix. Thus, the non-dimensional uniform mean flow equations in non-conservative form are given by;

$$
\begin{gathered}
\frac{\partial u^{\prime}}{\partial t}+M_{x} \frac{\partial u^{\prime}}{\partial x}+M_{y} \frac{\partial u^{\prime}}{\partial y}+\frac{\partial p^{\prime}}{\partial x}=0 \\
\frac{\partial v^{\prime}}{\partial t}+M_{x} \frac{\partial v^{\prime}}{\partial x}+M_{y} \frac{\partial v^{\prime}}{\partial y}+\frac{\partial p^{\prime}}{\partial y}=0 \\
\frac{\partial p^{\prime}}{\partial t}+M_{x} \frac{\partial p^{\prime}}{\partial x}+M_{y} \frac{\partial p^{\prime}}{\partial y}+\left(\frac{\partial u^{\prime}}{\partial x}+\frac{\partial v^{\prime}}{\partial y}\right)=0 \\
\frac{\partial \rho^{\prime}}{\partial t}+M_{x} \frac{\partial \rho^{\prime}}{\partial x}+M_{y} \frac{\partial \rho^{\prime}}{\partial y}+\left(\frac{\partial u^{\prime}}{\partial x}+\frac{\partial v^{\prime}}{\partial y}\right)=0
\end{gathered}
$$

In conservative form, the flux terms can be grouped as follows;

$$
\frac{\partial}{\partial t} Q+\frac{\partial}{\partial x} F+\frac{\partial}{\partial y} G=0
$$

where;

$$
Q=\left[\begin{array}{l}
\rho^{\prime} \\
u^{\prime} \\
v^{\prime} \\
p^{\prime}
\end{array}\right] ; \quad E=\left[\begin{array}{l}
M_{x} \rho^{\prime}+u^{\prime} \\
M_{x} u^{\prime}+p^{\prime} \\
M_{x} v^{\prime} \\
M_{x} p^{\prime}+u^{\prime}
\end{array}\right] ; \quad F=\left[\begin{array}{l}
M_{y} \rho^{\prime}+v^{\prime} \\
M_{y} u^{\prime} \\
M_{y} v^{\prime}+p^{\prime} \\
M_{y} p^{\prime}+v^{\prime}
\end{array}\right]
$$


in which $u^{\prime}$ and $v^{\prime}$ are the velocity perturbations in the $x$ and, $y$ directions, respectively; $p^{\prime}$ is the pressure; $\rho$ is the density; and $M_{x}$ and $M_{y}$ are expressed as,

$$
M_{x}=M \cos \alpha, M_{y}=M \sin \alpha
$$

where $\alpha$ is the angle between the direction of mean flow and the $x$-axis and, $M$ is the Mach number. $Q$, the solution vector, represents the acoustic perturbation in density, velocity and pressure. $M_{x}$ and $M_{y}$ are the mean flow velocity components normalized by the acoustic speed. The flow variables are written as the sum of a mean quantity and a perturbation quantity.

$$
\begin{gathered}
\rho=\bar{\rho}_{0}+\rho^{\prime} \\
u=\bar{u}_{0}+u^{\prime} \\
v=\bar{v}_{0}+v^{\prime} \\
p=\bar{p}_{0}+p^{\prime}
\end{gathered}
$$

Providing the mean flow Mach number, $M$, the perturbation quantities can be solved in a straight forward manner from Equation (2.28). In particular, this thesis deals with subsonic flows, i.e., $M<1$. 


\section{CHAPTER 3}

\section{GOVERNING EQUATIONS WITH PML}

\subsection{Definition of PML}

PML, the acronym for perfectly matched layer, is one of the popular emerging absorbing layer techniques. It is a region with a width of a few grid points, called the PML domain, introduced adjacent to artificial boundaries, such as radiation and out-flow boundaries. In the PML domain, the perfectly matched layer equations are constructed in such a way that out-going waves are absorbed without reflection, when they impinge on the interface between the PML and the interior domain. Initially, Berenger [2] introduced a perfectly matched layer technique for absorbing electromagnetic waves in a finite difference method of solving Maxwell equations. Hu later extended this for both linear and non-linear Euler and Navier-Stokes equations [6, 10-21].

$\mathrm{Hu}$ [10] has shown that the theoretical reflection coefficients for incident linear waves at an interface between the interior domain and a PML domain are zero. Thus, the amplitudes of waves that enter the PML domain decrease exponentially. It has also been shown that it is truly independent of the angle of incidence and frequency of the waves, [10] which is the main objective of applying the PML technique. It was further proved that the proposed PML equations [10] are capable of absorbing the out-going acoustic, vorticity and entropy waves without reflection for any angle of incidence and frequency of the waves, and the absorption rate is also independent of the wave frequency or wavelength. Thus, in numerical calculations, the computational domain is divided into the interior domain, where the Euler equations are applied, and the PML domains, where the PML equations are applied. The typical computational domain with 
a PML is shown in Figure 3.1. The governing equations in the PML domain are obtained by splitting the Euler equations in the coordinate directions. The absorption coefficients are introduced in each split direction. In fact, the solution in the PML domain has no physical significance, since it only helps to prevent the contamination of the solution in the interior domain.

\begin{tabular}{|c|c|c|c|}
\hline$\sigma_{x}, \sigma_{y}$ & $\sigma_{y}$ & PML & $\sigma_{x}, \sigma_{y}$ \\
\hline$\sum_{\Lambda}$ & INTERIOR DOMAIN & & $\sum_{0}^{+}$ \\
\hline$\sigma_{x}$ & - Mean & & $\sigma_{x}$ \\
\hline$\sigma_{x}, \sigma_{y}$ & $\sigma_{y}$ & PML & $\sigma_{x}, \sigma_{y}$ \\
\hline
\end{tabular}

Figure 3.1. Schematic of computational domain showing interior domain and PML domain on the boundary with absorption coefficients in the PML domain.

\subsection{PML Equations in Cartesian Coordinates}

Following the concept of Berenger [2], Hu [10] applied the PML technique to the Euler's equations. In his formulation of PML equations for the linearized Euler equations, Hu split each perturbation variable into two components with respect to the directions $x$, (denoted with a subscript " 1 ") and $y$ (denoted with a subscript " 2 "). Each component was subsequently integrated along its corresponding directions. Thus, the original system of four equations was rewritten into a system of eight equations. $\mathrm{Hu}$, in 
his formulation, split $u, v, p$, and $\rho$ of equation (2.5) to (2.8) into sub-components $u_{1}$, $u_{2}, v_{1}, v_{2}, p_{1}, p_{2}$, and $\rho_{1}, \rho_{2}$ and arrived at the following perfectly matched layer equations [11];

$$
\begin{gathered}
\frac{\partial u_{1}}{\partial t}+\sigma_{x} u_{1}=-\frac{\partial\left(p_{1}+p_{2}\right)}{\partial x}-M_{x} \frac{\partial\left(u_{1}+u_{2}\right)}{\partial x} \\
\frac{\partial u_{2}}{\partial t}+\sigma_{y} u_{2}=-M_{y} \frac{\partial\left(u_{1}+u_{2}\right)}{\partial y} \\
\frac{\partial v_{1}}{\partial t}+\sigma_{y} v_{1}=-\frac{\partial\left(p_{1}+p_{2}\right)}{\partial y}-M_{y} \frac{\partial\left(v_{1}+v_{2}\right)}{\partial y} \\
\frac{\partial v_{2}}{\partial t}+\sigma_{x} v_{2}=-M_{x} \frac{\partial\left(v_{1}+v_{2}\right)}{\partial x} \\
\frac{\partial p_{1}}{\partial t}+\sigma_{x} p_{1}=-\frac{\partial\left(u_{1}+u_{2}\right)}{\partial x}-M_{x} \frac{\partial\left(p_{1}+p_{2}\right)}{\partial x} \\
\frac{\partial p_{2}}{\partial t}+\sigma_{y} p_{2}=-\frac{\partial\left(v_{1}+v_{2}\right)}{\partial y}-M_{y} \frac{\partial\left(p_{1}+p_{2}\right)}{\partial y} \\
\frac{\partial \rho_{1}}{\partial t}+\sigma_{x} \rho_{1}=-\frac{\partial\left(u_{1}+u_{2}\right)}{\partial x}-M_{x} \frac{\partial\left(\rho_{1}+\rho_{2}\right)}{\partial x} \\
\frac{\partial \rho_{2}}{\partial t}+\sigma_{y} \rho_{2}=-\frac{\partial\left(v_{1}+v_{2}\right)}{\partial y}-M_{y} \frac{\partial\left(\rho_{1}+\rho_{2}\right)}{\partial y}
\end{gathered}
$$

where $\sigma_{x}$ and $\sigma_{y}$ have been introduced for the absorption of waves in the layer. These are called absorption coefficients and are assumed to be greater than or equal to zero. It is to be noted that, when $\sigma_{x}=\sigma_{y}=0$ and $\rho=\rho_{1}+\rho_{2}, u=u_{1}+u_{2}, v=v_{1}+v_{2}$, and $p=p_{1}+p_{2}$, equations (3.1) to (3.8) are reduced to the Euler equations, as shown in 
equations (2.24) to (2.27). Thus, the Euler equations are a special case of the PML equations.

In conservative form, the equations are given by

$$
\frac{\partial}{\partial t} Q+\frac{\partial}{\partial x} E+\frac{\partial}{\partial y} F+H=0
$$

where the flux vectors are

$$
Q=\left[\begin{array}{l}
\rho_{1} \\
\rho_{2} \\
u_{1} \\
u_{2} \\
v_{1} \\
v_{2} \\
p_{1} \\
p_{2}
\end{array}\right] ; \quad E=\left[\begin{array}{l}
M_{x} \rho^{\prime}+u^{\prime} \\
0 \\
M_{x} u^{\prime}+p^{\prime} \\
0 \\
0 \\
M_{x} v^{\prime} \\
M_{x} p^{\prime}+u^{\prime} \\
0
\end{array}\right] ; \quad F=\left[\begin{array}{l}
0 \\
M_{y} \rho^{\prime}+v^{\prime} \\
0 \\
M_{y} y^{\prime} \\
M_{y} v^{\prime}+p^{\prime} \\
0 \\
0 \\
M_{y} p^{\prime}+v^{\prime}
\end{array}\right] ; \quad H=\left[\begin{array}{l}
\sigma_{x} \rho_{1} \\
\sigma_{y} \rho_{2} \\
\sigma_{x} u_{1} \\
\sigma_{y} u_{2} \\
\sigma_{y} v_{1} \\
\sigma_{x} v_{2} \\
\sigma_{x} p_{1} \\
\sigma_{y} p_{2}
\end{array}\right]
$$

The source term, $H$, in equations (3.9) and (3.10) represents the damping term associated with the PML layers. The absorption coefficients $\sigma_{x}$ and $\sigma_{y}$ are varied across the layer in such a way that the coefficients are zero at the interface between the PML domain and the interior domain, and are maximum at the boundary of the PML domain. The variation of $\sigma_{x}$ and $\sigma_{y}$ in the PML is governed by the following relation:

$$
\sigma=\sigma_{m}\left(\frac{d}{D}\right)^{\beta}
$$

Where $D$ is the width of the PML domain, $d$ is the distance from its interface with the interior domain, $\sigma_{m}$ is the maximum value of $\sigma$, and $\beta$ is the damping rate. Alternatively, the PML domain can also be viewed as consisting of layers with constant $\sigma$, which varies linearly [10]. The maximum value of $\sigma$, however, will be restricted by 
the stability limit of the time integration scheme used, since an absorption term has been introduced in the PML equations. The absorption coefficients $\sigma_{x}$ and $\sigma_{y}$ are assumed to be greater than or equal to zero. Coefficient $\sigma_{x}$ is zero in a layer parallel to the $x$ direction, and coefficient $\sigma_{y}$ is zero in a layer parallel to $y$ direction. Both coefficients are greater than zero in the corner regions. Also, the larger the thickness, $D$ , of the PML, the better the wave absorption. However, as thickness increases, the computational time and effort required also increase correspondingly. At the end of a PML domain, certain boundary conditions, such as a solid wall condition or other radiation boundary condition can be applied. In numerical cases investigated in this research, extrapolation at the PML boundaries, i.e., the outer boundaries of the computational domain, is utilized. The parameters of the PML domains can be adjusted for desired absorption. They can also be determined independent of the size of the interior domain. Experiences [11] show that a value of $\sigma_{m} D /(\beta+1) \approx 8$ would provide satisfactory results. Typically, with a uniform grid of spacing $\Delta x$, if $D=10 \Delta x$ and $\beta=2$, then this results in $\sigma_{m} \Delta x=2.4$.

\subsection{Reflection and Transmission at Interfaces between Two PMLs}

In this section, the wave reflection and its transmission at an interface between the interior domain and a PML domain or between two PML domains are considered. Figure 3.2 shows the schematic of the angles of the acoustic waves at an interface [10]. Angles $\phi_{i}, \phi_{r}$, and $\phi_{t}$ are the angles made by the incident, reflected and transmitted waves, respectively, with the interior and PML domain interface. The absorption coefficients, $\sigma_{x}$ and $\sigma_{y}$, will be chosen in such a way that $\sigma_{y}$ is the same across an 
interface normal to $x$, and $\sigma_{x}$ is the same across an interface normal to $y$. It was already mentioned in section 3.2 that the Euler equations are a special case of PML equations when the absorption coefficients, $\sigma_{x}$ and $\sigma_{y}$, are zero. Consequently, $\sigma_{x}$ and $\sigma_{y}$ will be zero across an interface normal to $x$ or $y$ between an interior domain and a PML domain.

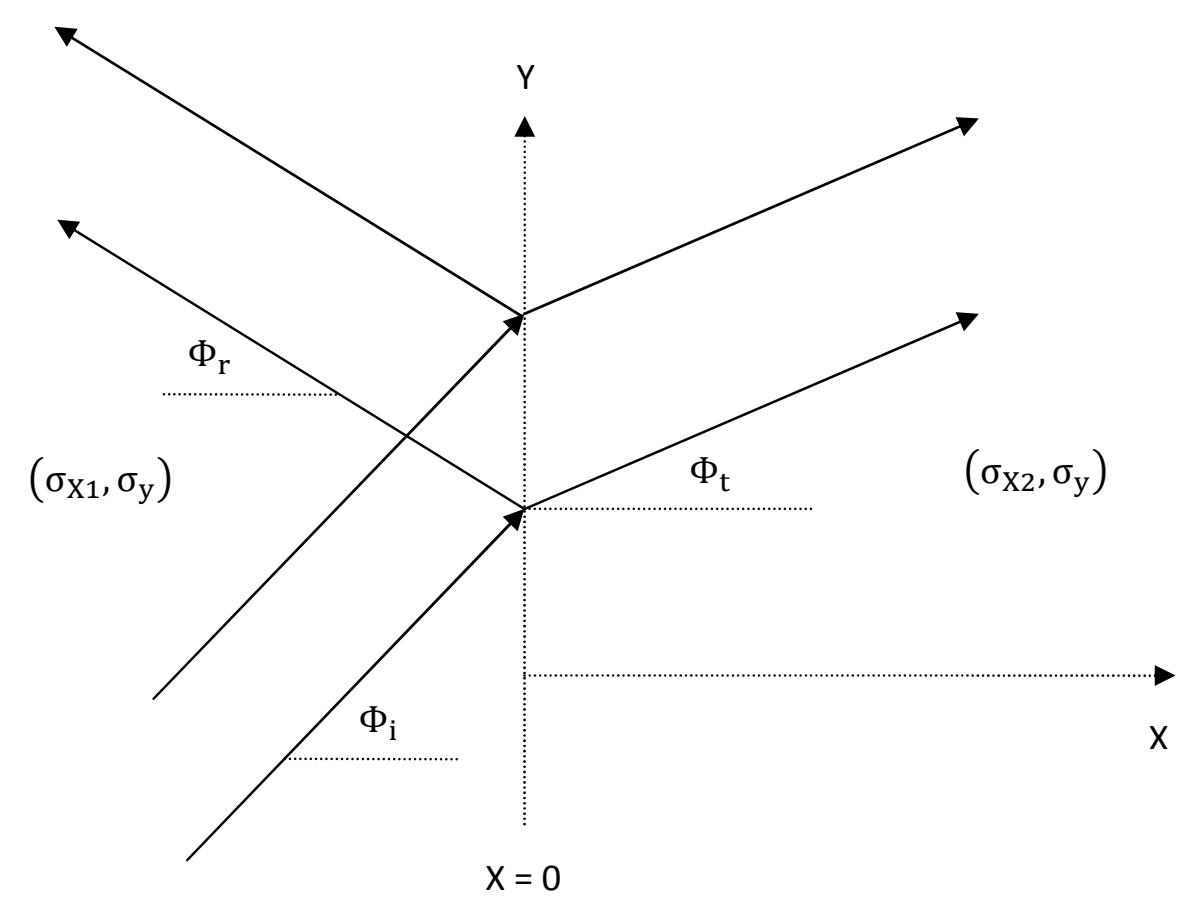

Figure 3.2. A schematic showing the angles of the acoustic wave at an interface.

To understand the behavior of acoustic, vorticity and entropy waves in the PML domain, the procedure employed by $\mathrm{Hu}[10]$ is reproduced. For his analysis on the incident, reflected, and transmitted waves, he expressed a plane wave in the PML domain as follows;

$$
\left[u_{1}, u_{2}, v_{1}, v_{2}, p_{1}, p_{2}, \rho_{1}, \rho_{2}\right]=\left[u_{10}, u_{20}, v_{10}, v_{20}, p_{10}, p_{20}, \rho_{10}, \rho_{20}\right] e^{i}\left(k_{x} x+k_{y} y-\omega t\right)
$$


where the subscript " 0 " denotes the amplitudes of the components. This is substituted in PML equations (3.13) to (3.20), where the mean flow is only in the direction of the $x$ axis with a Mach number of $M$.

$$
\begin{gathered}
\frac{\partial u_{1}}{\partial t}+\sigma_{x} u_{1}=-\frac{\partial\left(p_{1}+p_{2}\right)}{\partial x}-M \frac{\partial\left(u_{1}+u_{2}\right)}{\partial x} \\
\frac{\partial u_{2}}{\partial t}+\sigma_{y} u_{2}=0 \\
\frac{\partial v_{1}}{\partial t}+\sigma_{y} v_{1}=-\frac{\partial\left(p_{1}+p_{2}\right)}{\partial y} \\
\frac{\partial v_{2}}{\partial t}+\sigma_{x} v_{2}=-M \frac{\partial\left(v_{1}+v_{2}\right)}{\partial x} \\
\frac{\partial p_{1}}{\partial t}+\sigma_{x} p_{1}=-\frac{\partial\left(u_{1}+u_{2}\right)}{\partial x}-M \frac{\partial\left(p_{1}+p_{2}\right)}{\partial x} \\
\frac{\partial p_{2}}{\partial t}+\sigma_{y} p_{2}=-\frac{\partial\left(v_{1}+v_{2}\right)}{\partial y} \\
\frac{\partial \rho_{1}}{\partial t}+\sigma_{x} \rho_{1}=-\frac{\partial\left(u_{1}+u_{2}\right)}{\partial x}-M \frac{\partial\left(\rho_{1}+\rho_{2}\right)}{\partial x} \\
\frac{\partial \rho_{2}}{\partial t}+\sigma_{y} \rho_{2}=-\frac{\partial\left(v_{1}+v_{2}\right)}{\partial y}
\end{gathered}
$$

Thus, another set of equations,

$$
\begin{aligned}
& \left(\omega+i \sigma_{x}\right) u_{10}=k_{x}\left(p_{10}+p_{20}\right) \\
& \left(\omega+i \sigma_{y}\right) u_{20}=k_{x} M\left(u_{10}+u_{20}\right) \\
& \left(\omega+i \sigma_{y}\right) v_{10}=k_{y}\left(p_{10}+p_{20}\right) \\
& \left(\omega+i \sigma_{x}\right) v_{20}=k_{x} M\left(v_{10}+v_{20}\right)
\end{aligned}
$$




$$
\begin{gathered}
\left(\omega+i \sigma_{x}\right) p_{10}=k_{x}\left(u_{10}+u_{20}\right)+k_{x} M\left(p_{10}+p_{20}\right) \\
\left(\omega+i \sigma_{y}\right) p_{20}=k_{y}\left(v_{10}+v_{20}\right) \\
\left(\omega+i \sigma_{x}\right) \rho_{10}=k_{x}\left(u_{10}+u_{20}\right)+k_{x} M\left(p_{10}+p_{20}\right) \\
\left(\omega+i \sigma_{y}\right) \rho_{20}=k_{x}\left(v_{10}+v_{20}\right)
\end{gathered}
$$

is obtained. Hu shows that these equations support acoustic waves, when

$$
\left(\omega-k_{x} M+i \sigma_{x}\right) \neq 0
$$

and these equations support vorticity and entropy waves, when

$$
\left(\omega-k_{x} M+i \sigma_{x}\right)=0
$$

Based on these conditions $\mathrm{Hu}$ arrives at a solution for the plane wave, which is expressed as

$$
\left(\begin{array}{l}
u_{1} \\
u_{2} \\
v_{1} \\
v_{2} \\
p_{1} \\
p_{2} \\
\rho_{1} \\
\rho_{2}
\end{array}\right)=A\left(\begin{array}{l}
\cos \phi \\
M \cos ^{2} \phi \\
\sin \phi \\
M \cos \phi \sin \phi \\
\cos ^{2} \phi+M \cos \phi \\
\sin ^{2} \phi \\
\cos ^{2} \phi+M \cos \phi \\
\sin ^{2} \phi
\end{array}\right) e^{i \omega\left(\frac{\cos \phi}{1+M \cos \phi} x+\frac{\sin \phi}{1+M \cos \phi} y-t\right)} e^{-\frac{\sigma_{x} \cos \phi}{1+M \cos \phi} x-\frac{\sigma_{y} \sin \phi}{1+M \cos \phi} y}
$$

He shows that this expression represents a wave propagating with the speed of sound relative to the mean flow in the direction making an angle $\phi$ with respect to the $x$ axis, and he verifies this solution for the acoustic wave. He further adds that the magnitude of the wave decreases exponentially as it propagates in the $x$ or $y$ direction, when the absorption coefficients, $\sigma_{x}$ and $\sigma_{y}$, are not zero. 
In a similar fashion, Hu derives a solution for vorticity and entropy waves. The plane wave solution is thus expressed as

$$
\left(\begin{array}{l}
u_{1} \\
u_{2} \\
v_{1} \\
v_{2} \\
p_{1} \\
p_{2} \\
\rho_{1} \\
\rho_{2}
\end{array}\right)=A\left(\begin{array}{l}
0 \\
-B \sin \psi \\
0 \\
B \cos \psi \\
-B(\sin \psi) / M \\
B(\sin \psi) / M \\
C \\
B(\sin \psi) / M
\end{array}\right) e^{i \omega\left(\frac{1}{M} x+\frac{\tan \psi}{M} y-t\right)} e^{-\frac{\sigma_{y}}{M} x-\frac{\sigma_{y} \tan \psi}{M} y}
$$

In equation (3.32), the constants $B$ and $C$ independently represent the vorticity and the entropy waves, respectively. Since each component is of the form $f\left(\frac{x}{M}-t, y\right) e^{\frac{-\sigma_{x}}{M} x}$, the waves are convected with the mean flow at a Mach number equal to $M$. Hu also adds that the magnitude of the wave is decreased exponentially when $\sigma_{x}$ is greater than zero. It is also seen from the expression that the vorticity waves do not contribute to the $u_{1}$ and $v_{1}$ components in the PML domain. The individual solution for acoustic, vorticity, and entropy waves can be combined together to express the incident, reflected, and transmitted waves, as follows: 
(a) Incident wave:
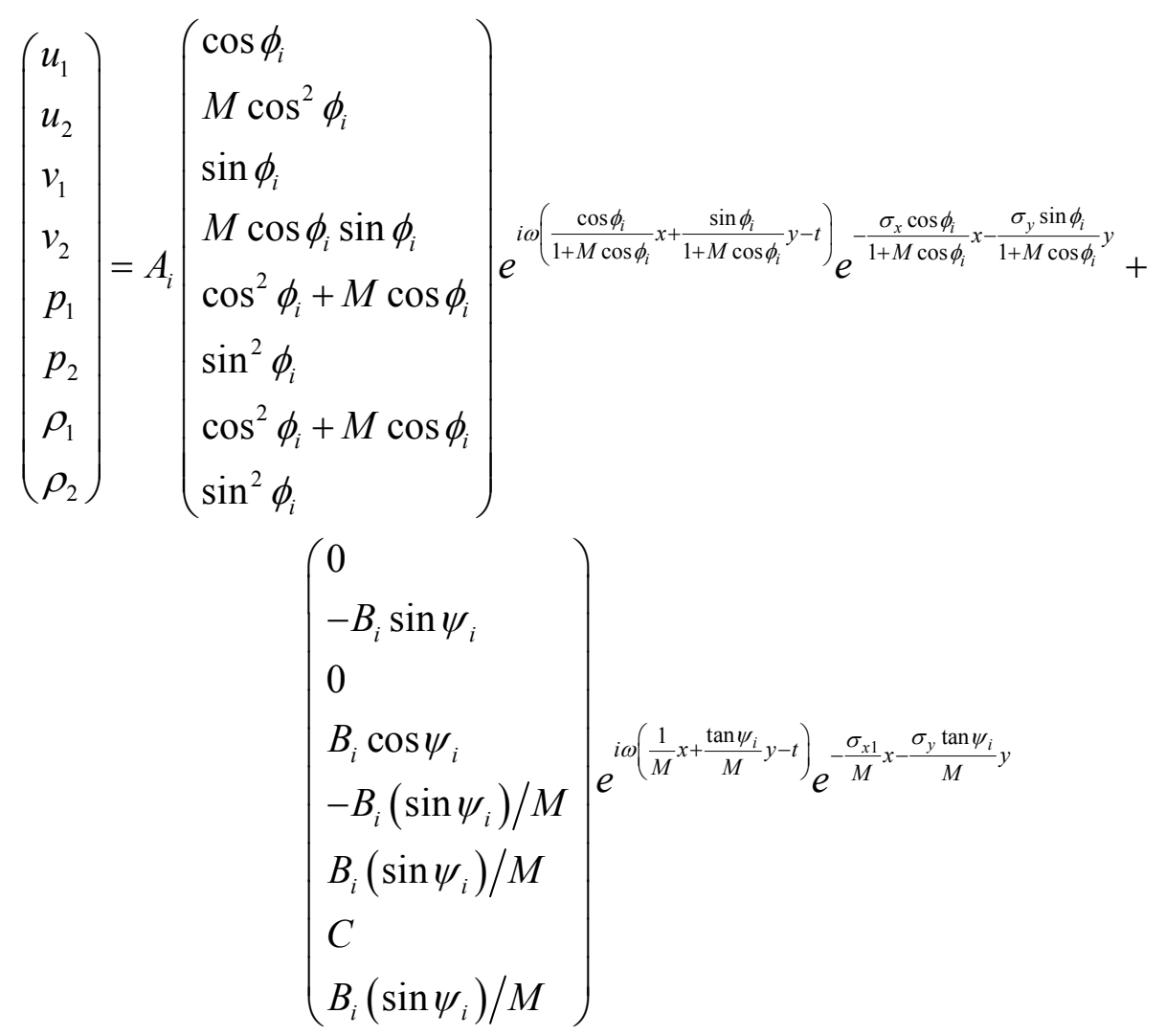

(b) Reflected wave:

$$
\left(\begin{array}{l}
u_{1} \\
u_{2} \\
v_{1} \\
v_{2} \\
p_{1} \\
p_{2} \\
\rho_{1} \\
\rho_{2}
\end{array}\right)=A_{r}\left(\begin{array}{l}
-\cos \phi_{r} \\
M \cos ^{2} \phi_{r} \\
\sin \phi_{r} \\
-M \cos \phi_{r} \sin \phi_{r} \\
\cos ^{2} \phi_{r}-M \cos \phi_{r} \\
\sin ^{2} \phi_{r} \\
\cos ^{2} \phi_{r}-M \cos \phi_{r} \\
\sin ^{2} \phi_{r}
\end{array}\right) e^{i \omega\left(-\frac{\cos \phi_{r}}{1-M \cos \phi_{r}} x+\frac{\sin \phi_{r}}{1-M \cos \phi_{r}} y-t\right)} e^{-\frac{\sigma_{x} \cos \phi_{r}}{1-M \cos \phi_{r}} x-\frac{\sigma_{y} \sin \phi_{r}}{1-M \cos \phi_{r}} y}
$$


(c) Transmitted wave:

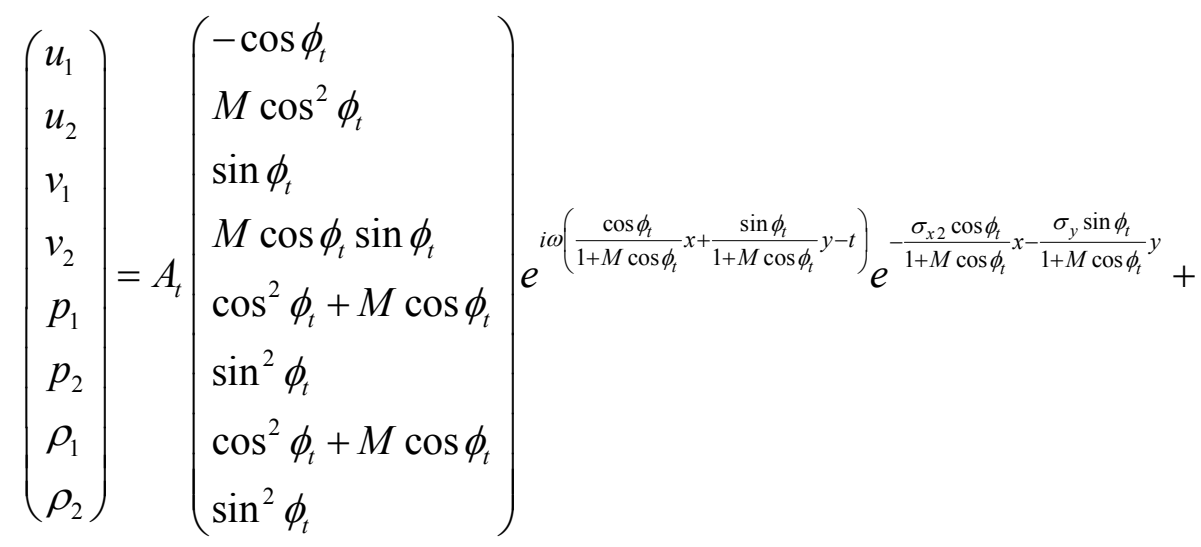

$$
\left(\begin{array}{l}
0 \\
-B_{t} \sin \psi_{t} \\
0 \\
B_{t} \cos \psi_{t} \\
-B_{t}\left(\sin \psi_{t}\right) / M \\
B_{t}\left(\sin \psi_{t}\right) / M \\
C_{t} \\
B_{t}\left(\sin \psi_{t}\right) / M
\end{array}\right) e^{i \omega\left(\frac{1}{M} x+\frac{\tan \psi_{t}}{M} y-t\right)} e^{-\frac{\sigma_{x 2} x-\frac{\sigma_{y} \tan \psi_{t}}{M} y}{M}}
$$

At the interface, it is imposed that $u_{1}+u_{2}, v_{1}+v_{2}, p_{1}+p_{2}$, and $\rho_{1}+\rho_{2}$ are continuous. For this continuity to be true for all values of $y$ along the interface, the coefficients of $y$ in the exponents of equations (3.33) to (3-35) must be the same for all incident, reflected, and transmitted waves. This results in

$$
\begin{gathered}
\frac{\sin \phi_{r}}{1-M \cos \phi_{r}}=\frac{\sin \phi_{i}}{1+M \cos \phi_{i}} \\
\frac{\sin \phi_{t}}{1+M \cos \phi_{t}}=\frac{\sin \phi_{i}}{1+M \cos \phi_{i}} \\
\tan \psi_{t}=\tan \psi_{i}
\end{gathered}
$$


Assuming $\tan \psi_{t}=\frac{\sin \phi_{i}}{1+M \cos \phi_{i}}$, it was found that

$$
\phi_{r}=2 \tan ^{-1}\left(\frac{1-M}{1+M} \tan \frac{\phi_{i}}{2}\right), \quad \phi_{t}=\phi_{i} \quad, \quad \psi_{t}=\psi_{i}
$$

By the continuity of $u_{1}+u_{2}, v_{1}+v_{2}, p_{1}+p_{2}$, and $\rho_{1}+\rho_{2}$,

$$
\begin{gathered}
A_{i}\left(1+M \cos \phi_{i}\right) \cos \phi_{i}-A_{r}\left(1-M \cos \phi_{r}\right) \cos \phi_{r}-B_{i} \sin \psi_{i} \\
=A_{t}\left(1+M \cos \phi_{t}\right) \cos \phi_{t}-B_{t} \sin \psi_{t} \\
A_{i}\left(1+M \cos \phi_{i}\right) \sin \phi_{i}-A_{r}\left(1-M \cos \phi_{r}\right) \sin \phi_{r}+B_{i} \cos \psi_{i} \\
=A_{t}\left(1+M \cos \phi_{t}\right) \sin \phi_{t}+B_{t} \cos \psi_{t} \\
A_{i}\left(1+M \cos \phi_{i}\right)+A_{r}\left(1-M \cos \phi_{r}\right)=A_{t}\left(1+M \cos \phi_{t}\right)
\end{gathered}
$$

and

$$
\begin{aligned}
A_{i}\left(1+M \cos \phi_{i}\right)+A_{r}\left(1-M \cos \phi_{r}\right)+B_{i} \frac{\sin \psi_{i}}{M}+C_{i} & \\
= & A_{t}\left(1+M \cos \phi_{t}\right)+B_{t} \frac{\sin \psi_{t}}{M}+C_{t}
\end{aligned}
$$

By substituting $\phi_{r}, \phi_{t}$, and $\psi_{t}$ into equations (3.40) to (3.43), a linear system of homogeneous equations for $A_{i}-A_{t}, A_{r}, B_{i}-B_{t}$, and $C_{i}-C_{t}$ can be obtained. The coefficient determinant of this system was found to be

$$
2\left(1+M \cos \phi_{i}\right)\left(1-M \cos \phi_{r}\right) \cos \left(\frac{\phi_{i}+\phi_{r}}{2}\right) \cos \left(\psi_{i}-\frac{\phi_{i}-\phi_{r}}{2}\right)
$$

Equation (3.44) is not zero for any angle of incidence and, thus, the only solution is

$$
A_{r}=0 \quad, \quad A_{t}=A_{i} \quad, \quad B_{t}=B_{i}, C_{t}=C_{i}
$$

From equations (3.39) and (3.45), Hu demonstrated that at an interface between two PML domains downstream normal to the $x$ axis with absorption coefficients $\left(\sigma_{x 1}, \sigma_{y}\right)$ 
and $\left(\sigma_{x 2}, \sigma_{y}\right)$, respectively, the reflection is null and the transmitted waves maintain the same direction and amplitude as the incident waves at an interface. This has been shown to be independent of the angle of incidence and frequency of the waves. A similar analysis for a mean flow in an arbitrary direction, as defined by PML equations (3.1) to (3.8) is also presented by $\mathrm{Hu}[11]$.

\subsection{PML Equations in Generalized Coordinates}

It is considerably less complex to generate a rectangular computational domain and solve the finite differential equations with appropriate boundary conditions where the grid points of the boundaries may be specified as coincident with the boundaries of the physical domain. However, in reality, the majority of the physical domains of interest are non-rectangular. In order to take care of these irregular boundaries, some sort of interpolation for the implementation of the boundary conditions will be necessary. However, this approach is not an efficient one, as the boundary conditions have a dominant influence on the numerical solution. Further, non-equal step sizes must be used in the approximations of the numerical schemes, which may create further complications. This necessitates a transformation from physical space to computational space by specifying a generalized coordinate system and mapping the non-rectangular grid system in the physical space to a rectangular uniform grid in the computational space. Written in the conservative form, the governing equation, equation (3.9), in the PML domain for uniform mean flow in the generalized coordinates is [7]

$$
\frac{\partial}{\partial \tau} \bar{Q}+\frac{\partial}{\partial \xi} \bar{E}+\frac{\partial}{\partial \eta} \bar{F}+\bar{H}=0
$$

where 


$$
\begin{aligned}
& \bar{Q}=\frac{1}{J}\left[\begin{array}{l}
\rho_{1} \\
\rho_{2} \\
u_{1} \\
u_{2} \\
v_{1} \\
v_{2} \\
p_{1} \\
p_{2}
\end{array}\right] \quad ; \quad E=\frac{1}{J}\left[\begin{array}{l}
\xi_{x}\left(M_{x} \rho^{\prime}+u^{\prime}\right)+\xi_{y}\left(M_{x} \rho^{\prime}+v^{\prime}\right) \\
0 \\
\xi_{x}\left(M_{x} u^{\prime}+p^{\prime}\right)+\xi_{y}\left(M_{y} u^{\prime}\right) \\
0 \\
0 \\
\xi_{x}\left(M_{x} v^{\prime}\right)+\xi_{y}\left(M_{x} v^{\prime}+p^{\prime}\right) \\
\xi_{x}\left(M_{x} p^{\prime}+u^{\prime}\right)+\xi_{y}\left(M_{y} p^{\prime}+v^{\prime}\right) \\
0
\end{array}\right] \\
& F=\frac{1}{J}\left[\begin{array}{l}
0 \\
\eta_{x}\left(M_{x} \rho^{\prime}+u^{\prime}\right)+\eta_{y}\left(M_{y} \rho^{\prime}+v^{\prime}\right) \\
0 \\
\eta_{x}\left(M_{x} u^{\prime}+p^{\prime}\right)+\eta_{y}\left(M_{y} u^{\prime}\right)+\eta_{y}\left(M_{y} v^{\prime}+p^{\prime}\right) \\
0 \\
0 \\
\eta_{x}\left(M_{x} p^{\prime}+u^{\prime}\right)+\eta_{y}\left(M_{y} p^{\prime}+v^{\prime}\right)
\end{array}\right] ; \quad H=\frac{1}{J}\left[\begin{array}{l}
\sigma_{\xi} \rho_{1} \\
\sigma_{\eta} \rho_{2} \\
\sigma_{\xi} u_{1} \\
\sigma_{\eta} u_{2} \\
\sigma_{\eta} v_{1} \\
\sigma_{\xi} v_{2} \\
\sigma_{\xi} p_{1} \\
\sigma_{\eta} p_{2}
\end{array}\right]
\end{aligned}
$$

\subsection{Filtering}

$\mathrm{Hu}$ [11] used artificial filtering in order to overcome the instability. He thought that instability resulted from the numerical scheme that he used. However, subsequently it was shown by Tam and Auriault [26] that instability is not due to the numerical scheme but rather due to the splitting of equations, as introduced by Berenger. It was ascertained mathematically by Abarbanel et al. [27] that Berenger's formulation of PML is not well-posed. In the present work, filtering is not used in the numerical scheme. 


\section{CHAPTER 4}

\section{NUMERICAL SCHEME FOR TIME DOMAIN}

In computational fluid dynamics, the governing partial differential equations that define a given problem are solved numerically. The literature is abundant with a variety of schemes to solve partial differential equations. The present work considers a flow involving acoustic perturbations defined by the linearized Euler equations for a mean flow that is hyperbolic in nature. A number of finite difference approximations have been studied by various investigators. In this work, a scheme that can produce highly accurate solution with minimal dissipation and dispersion errors was required, as the acoustic waves were dispersive in nature. The acoustic perturbations were solved in the time domain with the linearized Euler equations for a uniform mean flow.

\subsection{Grid Structure}

The linearized Euler equations for a uniform mean flow are solved in two types of grid structures: (1) uniform grid structure, and (2) non-uniform grid structure. The uniform grid structure was obtained by having equal spacing in both the $x$ and $y$ directions. Throughout the present work, for the uniform grid structure, a spacing of $\Delta x=\Delta y=1.0$ is used as shown in Figure 4.1 (a). Thus, the uniform grid structure is geometrically a square domain. The geometrical definition of non-uniform grid structure is depicted in Figure 4.1 (b). This grid has a constant spacing, $\Delta x=1.0$, in the $x$ direction. $\Delta y=1.5$ only along $x=-50$ and the spacing is non-uniform for all other $x$. The coordinates of the right top corner of the domain is calculated using the angle $\theta$ in order to estimate the height of the domain at $x=50$. The coordinates of the points along $x=50$ are calculated using this length and the number of grid points. The slopes of lines connecting two 
respective grid points along $x=-50$ and $x=50$ are calculated for each grid line along $y$ in order to find the coordinates of the remaining grid points within the domain. The purpose of non-uniform grid structure was previously discussed in section 3.4. Thus, the linearized Euler equations are solved for a uniform mean flow using the PML formulation in generalized coordinates.

(a)

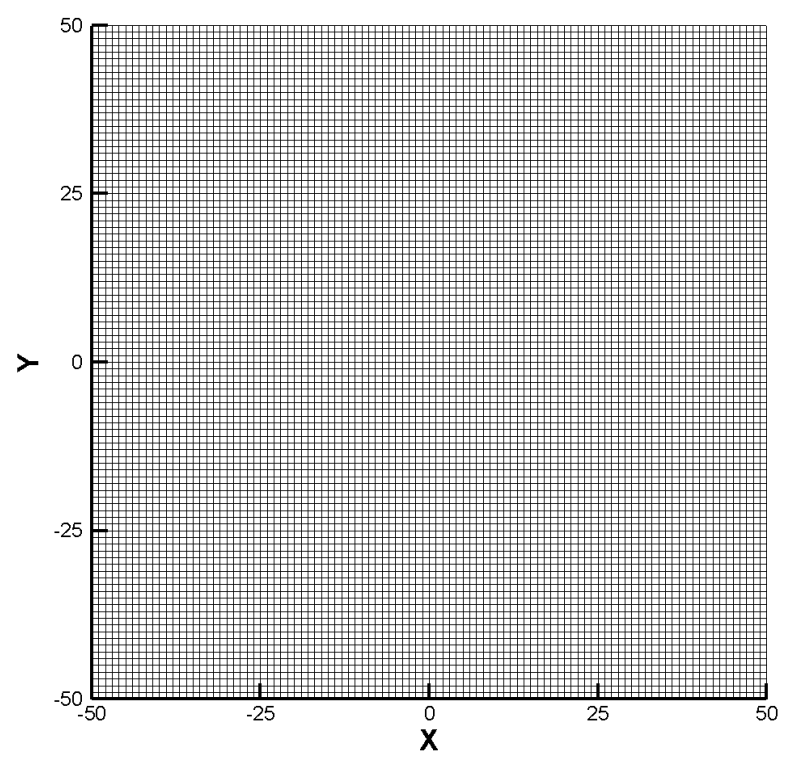

(b)

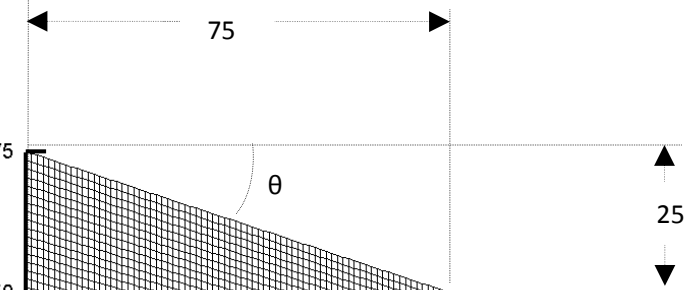

25

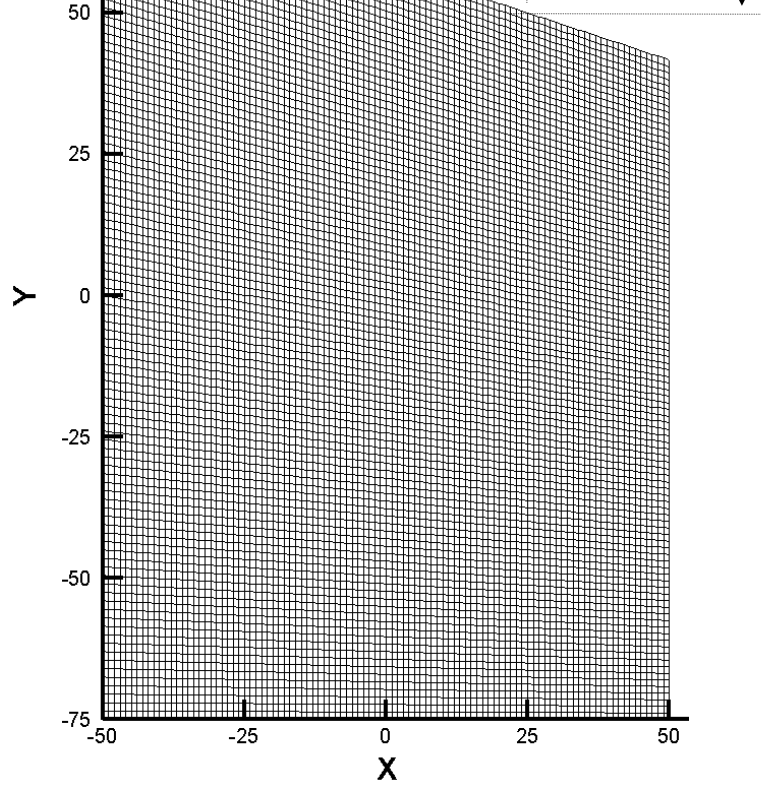

Figure 4.1. (a) Uniform grid structure, (b) Non-uniform grid structure

\subsubsection{Spatial Discretization}

Recall that the linearized Euler equation for a mean flow given by Equation (2.28) was presented in section 2.4.

$$
\frac{\partial}{\partial t} Q+\frac{\partial}{\partial x} E+\frac{\partial}{\partial y} F=0
$$


As per the discussion in section 3.4, equation (4.1) was solved in generalized coordinates, whereby the governing equation given by Equation (3.46) is;

$$
\frac{\partial}{\partial \tau} \bar{Q}+\frac{\partial}{\partial \xi} \bar{E}+\frac{\partial}{\partial \eta} \bar{F}+\bar{H}=0
$$

where

$$
\bar{Q}=\frac{Q}{J} \quad, \quad \bar{E}=\frac{1}{J}\left(\xi_{x} E+\xi_{y} F\right), \bar{F}=\frac{1}{J}\left(\eta_{x} E+\eta_{y} F\right)
$$

The metrics $\xi_{x}, \xi_{y}, \eta_{x}$, and $\eta_{y}$ were approximated by sixth-order compact differencing scheme. A five-point stencil was used where the increased width minimizes the dispersion and dissipation errors present in the traditional difference schemes. A general five-point formulation for the approximation of the first derivative can be written as [1];

$$
\beta f_{i-2}^{\prime}+\alpha f_{i-1}^{\prime}+f_{i}^{\prime}+\alpha f_{i+1}^{\prime}+\beta f_{i+2}^{\prime}=a \frac{f_{i+1}-f_{i-1}}{2 \Delta x}+b \frac{f_{i+2}-f_{i-2}}{4 \Delta x}+c \frac{f_{i+3}-f_{i-3}}{6 \Delta x}
$$

where $\Delta x$ is the grid spacing in the $i$ direction over a uniformly spaced grid. In equation (4.4), the coefficients $\beta, \alpha, a, b$, and $c$ are constants. The relations between the coefficients are established by matching of the coefficients obtained by the substitution of the Taylor series expansions. Depending on how $\alpha$ and $\beta$ are specified, either a tridiagonal or a pentadiagonal system was generated from equation (4.4). By specifying that $\beta=0, a=\frac{2}{3}(\alpha+2), b=\frac{1}{3}(4 \alpha-1)$, and $c=0$, a tridiagonal system was obtained where the truncation error from the Taylor series substitution is

$$
\text { Truncation Error }=\frac{4}{5 !}(3 \alpha-1)(\Delta x)^{4} f_{i}^{v}
$$


By choosing different values of $\alpha$, the tridiagonal system with a different order of accuracy can be obtained. For $\alpha=1 / 3$ and the resulting values of $a=14 / 9$ and $b=1 / 9$, the numerical scheme becomes formally sixth order, due to the disappearance of the leading term in the truncation error [1]. Now, Equation (4.4) becomes

$$
\frac{1}{3} f_{i-1}^{\prime}+f_{i}^{\prime}+\frac{1}{3} f_{i+1}^{\prime}=\frac{14}{9} \frac{f_{i+1}-f_{i-1}}{2 \Delta x}+\frac{1}{9} \frac{f_{i+2}-f_{i-2}}{4 \Delta x}
$$

At the boundaries, considering points $i=1$ and $i=I M$ to be the boundary points, the general expressions for one sided approximations are

$$
\begin{gathered}
f_{1}^{\prime}+\alpha f_{2}^{\prime}=\frac{1}{\Delta x}\left(a f_{1}+b f_{2}+c f_{3}+d f_{4}\right) \\
f_{I M}^{\prime}+\alpha f_{I M 1}^{\prime}=\frac{1}{\Delta x}\left(a f_{I M}+b f_{I M 1}+c f_{I M 2}+d f_{I M 3}\right)
\end{gathered}
$$

where $I M 1=I M-1, I M 2=I M-2$, and $I M 3=I M-3$.

For a fourth-order approximation, the values of constants are given by

$$
\alpha=3, \quad a=\frac{-17}{6}, b=\frac{3}{2}, c=\frac{3}{2}, \quad d=\frac{-1}{6}
$$

The truncation error for these approximations is

$$
\text { Truncation Error }=\frac{6}{5 !}(\Delta x)^{4} f_{1}^{v}
$$

\subsubsection{Temporal Integration}

To increase the accuracy of the solution, a higher-order temporal discretization was used in conjunction with the higher-order spatial discretization [31]. A Modified fourth-order Runge-Kutta scheme was used for temporal integration. This scheme utilizes the weighted average of several solutions over the interval $\Delta t$ in order to 
improve accuracy of solution [32]. The modified fourth-order Runge-Kutta scheme is given by

$$
\begin{gathered}
\bar{Q}_{i, j}^{(0)}=\bar{Q}_{i, j}^{n} \\
\bar{Q}_{i, j}^{(1)}=\bar{Q}_{i, j}^{n}-\frac{\Delta t}{4}\left[\left(\frac{\partial \bar{E}}{\partial \xi}\right)_{i, j}^{(0)}+\left(\frac{\partial \bar{F}}{\partial \eta}\right)_{i, j}^{(0)}+\alpha \bar{H}_{i, j}^{(0)}\right] \\
\bar{Q}_{i, j}^{(2)}=\bar{Q}_{i, j}^{n}-\frac{\Delta t}{3}\left[\left(\frac{\partial \bar{E}}{\partial \xi}\right)_{i, j}^{(1)}+\left(\frac{\partial \bar{F}}{\partial \eta}\right)_{i, j}^{(1)}+\alpha \bar{H}_{i, j}^{(1)}\right] \\
\bar{Q}_{i, j}^{(3)}=\bar{Q}_{i, j}^{n}-\frac{\Delta t}{4}\left[\left(\frac{\partial \bar{E}}{\partial \xi}\right)_{i, j}^{(2)}+\left(\frac{\partial \bar{F}}{\partial \eta}\right)_{i, j}^{(2)}+\alpha \bar{H}_{i, j}^{(2)}\right] \\
\bar{Q}_{i, j}^{(4)}=\bar{Q}_{i, j}^{n}-\frac{\Delta t}{4}\left[\left(\frac{\partial \bar{E}}{\partial \xi}\right)_{i, j}^{(3)}+\left(\frac{\partial \bar{F}}{\partial \eta}\right)_{i, j}^{(3)}+\alpha \bar{H}_{i, j}^{(3)}\right] \\
\bar{Q}_{i, j}^{(n+1)}=\bar{Q}_{i, j}^{(4)}
\end{gathered}
$$

The superscripts $n$ and $n+1$ refer to the time levels, and the superscripts within the parentheses refer to the values at the intermediate stages of the Runge-Kutta scheme. The term $\bar{H}_{i, j}^{(0)}$ is the damping term evaluated at the first stage of the Runge-Kutta scheme. 


\section{CHAPTER 5}

\section{EXAMPLES AND RESULTS IN TIME DOMAIN}

Boundary conditions are of paramount importance in computational fluid dynamics problems, since the accuracy of the numerical solution relies on the conditions that must be specified on the boundaries of the computational domains. This is especially crucial in the case of open-domain problems, where the domain of interest is truncated using artificial boundaries. Therefore, when the waves leave the domain, there must be assurance that these boundary conditions do not cause any reflections in order to avoid a spurious solution. Thus, the application of artificial boundary conditions to accomplish a reflection-free boundary becomes critical. A variety of artificial boundary conditions are available in the literature which is generally called as non-reflecting boundary conditions. There are two ways to absorb the outgoing waves/disturbances: "hard-absorbing" NRBCs and "soft-absorbing" NRBCs. In hard-absorbing boundary conditions, the given boundary conditions are made admissible by using the characteristics method. In soft-absorbing boundary conditions, either a buffer zone technique with increased dissipation, grid stretching, or the recently developed perfectly matched layer technique is used. The soft-absorbing technique is imposed in the matching/absorbing layer at the boundary in order to guarantee the exponential decay of the disturbances in the layer [4]. Since the PML boundary treatment is the primary focus of the current work, its importance is established in this section. 


\subsection{Validation of PML Formulation}

Before showing the details of how PML formulation compares with numerical solutions obtained without PML, the formulation is validated by considering the twodimensional acoustic and vorticity disturbances [10]. The mean flow is in the direction of the $x$ - axis with $M=0.5$. The computational domain, $[-60,60] \times[-60,60]$, is a uniform grid with a spacing of $\Delta x=\Delta y=1$ and a PML domain of ten grid points around the four sides, as shown in Figure 5.1(a) and (b). Initially, the acoustic pulse is located at $\left(x_{a}, y_{a}\right)=(-25,0)$, and the vorticity and entropy pulses are located at $\left(x_{b}, y_{b}\right)=(25,0)$.

The values of $\sigma_{m}=\beta=2$, as used by $\mathrm{Hu}$ [10], were selected for the present calculations, where the initial conditions are adopted from the category three of Benchmark Problems of Computational Aeroacoustics [33] and are given by the following equations:

$$
\begin{gathered}
\rho=\exp \left[-(\ln 2)\left(\frac{\left(x-x_{a}\right)^{2}+\left(y-y_{a}\right)^{2}}{9}\right)\right]+0.1 \exp \left[-(\ln 2)\left(\frac{\left(x-x_{b}\right)^{2}+\left(y-y_{b}\right)^{2}}{16}\right)\right] \\
p=\exp \left[-(\ln 2)\left(\frac{\left(x-x_{a}\right)^{2}+\left(y-y_{a}\right)^{2}}{9}\right)\right] \\
u=0.05\left(y-y_{b}\right) \exp \left[-(\ln 2)\left(\frac{\left(x-x_{b}\right)^{2}+\left(y-y_{b}\right)^{2}}{16}\right)\right] \\
v=0.05\left(x-x_{b}\right) \exp \left[-(\ln 2)\left(\frac{\left(x-x_{b}\right)^{2}+\left(y-y_{b}\right)^{2}}{16}\right)\right]
\end{gathered}
$$

These equations include an acoustic pulse centered at $\left(x_{a}, y_{a}\right)$ and vorticity and entropy pulse both centered at $\left(x_{b}, y_{b}\right)$. In his calculations, Hu [10] used a fourth-order seven- 
point explicit central difference scheme for the spatial discretization and a fourth-order Runge-Kutta scheme optimized for low-dissipation and low-dispersion errors for the time integration. In the present case, a sixth-order compact differencing scheme was used for the spatial discretization, and a fourth-order Runge-Kutta scheme with a time step size of 0.05 was used for the time integration.

(a)

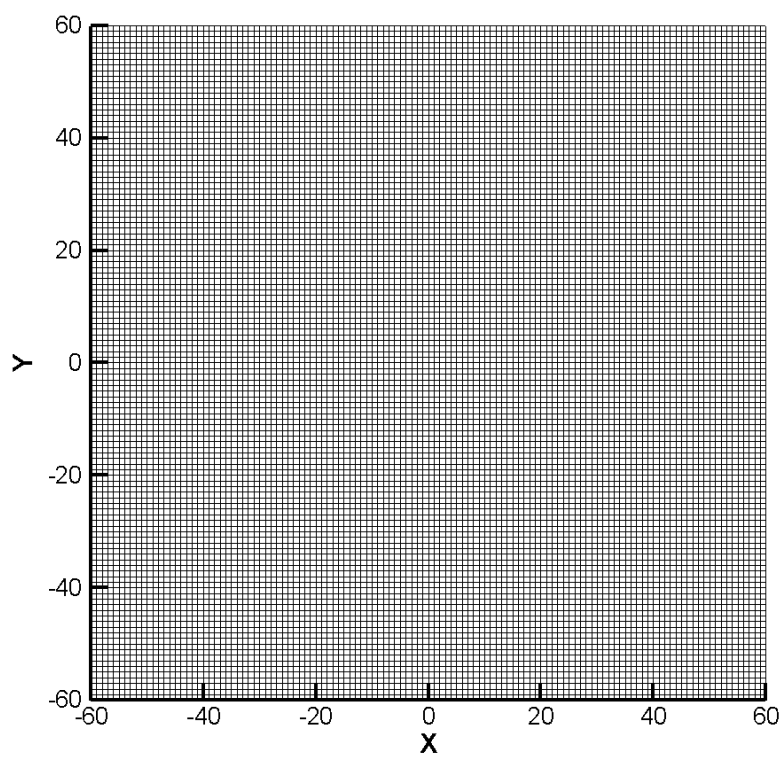

(b)

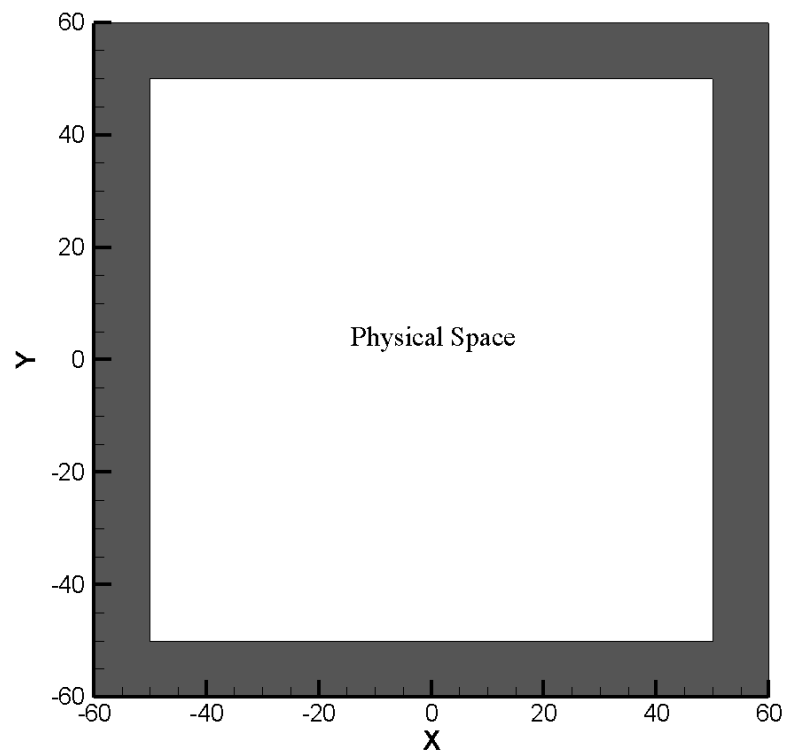

Figure 5.1. Computational domain for uniform grid: (a) grid structure, (b) PML domain and physical space. Number of layers $=10$.

The density contours and $u$ velocity contours at various time levels are presented in Figures 5.2 and 5.3. Figure 5.4 shows the density along line $y=0$ at three time levels. These results compare well with that of Hu's work [10]. Figure 5.5 shows the density contours in the vicinity of the Euler and PML domain interfaces. Here, it can be seen that the waves are absorbed in the PML domain. It must be understood that the solution in the PML domain has no physical significance, since this domain is used to 
absorb the waves so that they are not reflected at the boundaries back into the computational domain.

(a)

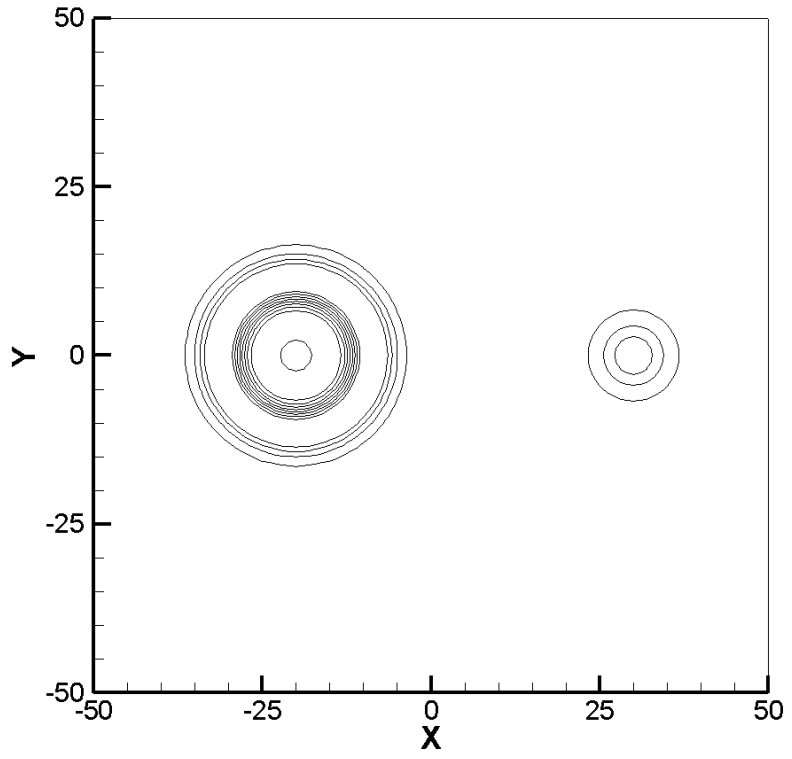

(c)

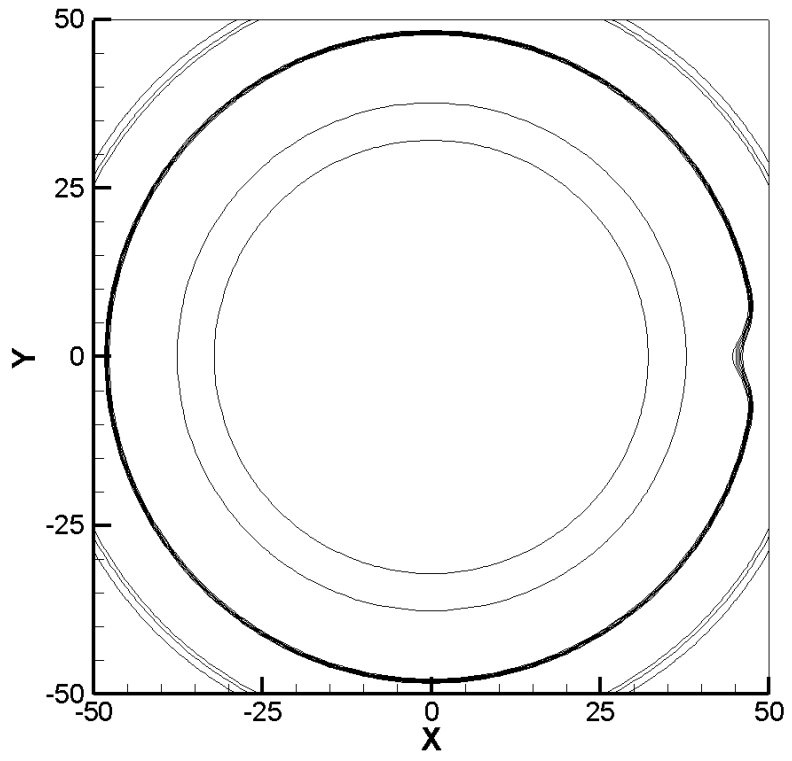

(b)

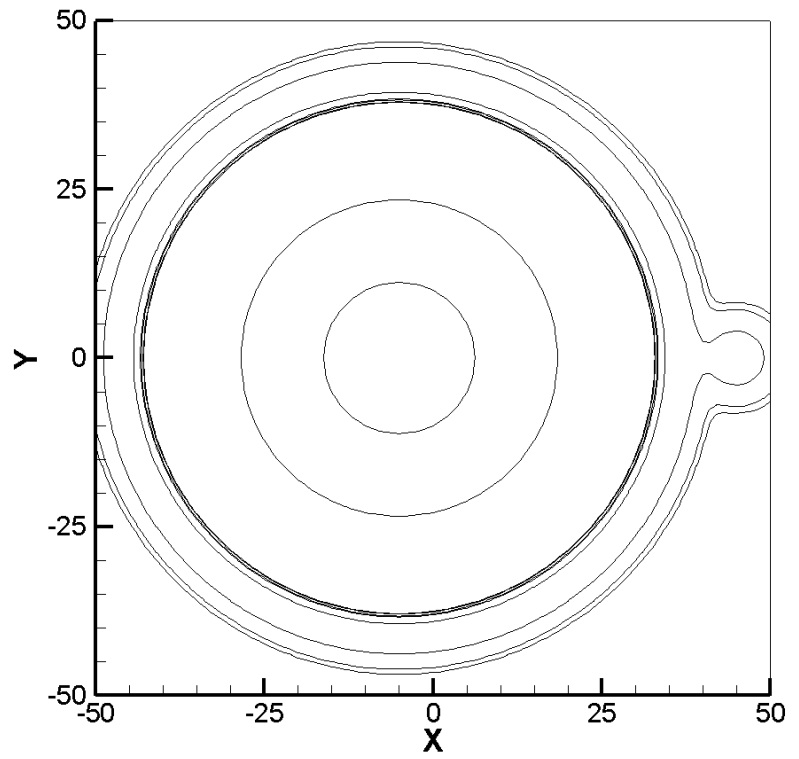

(d)

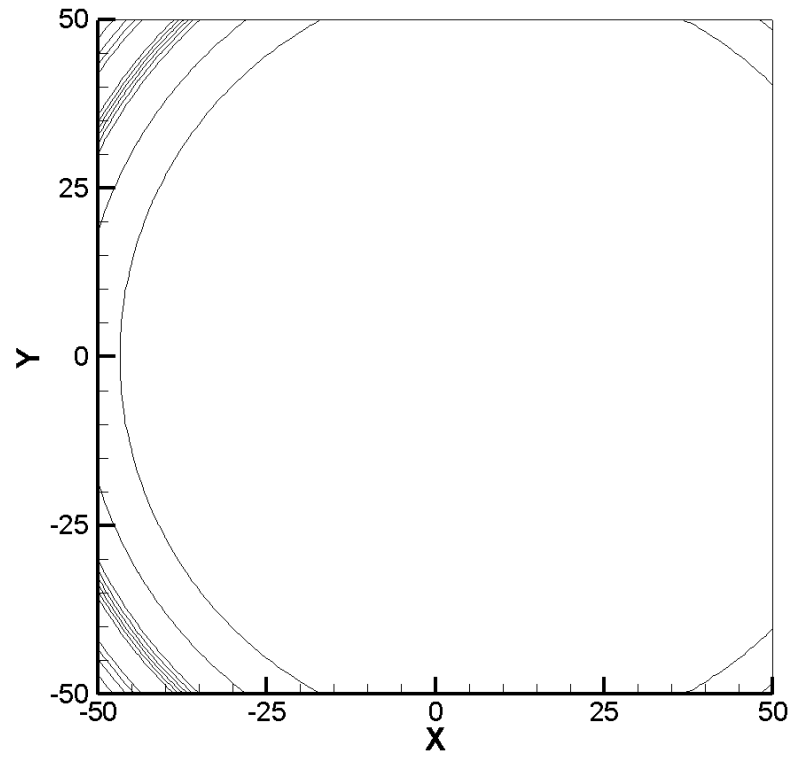

Figure 5.2. Density contours at values of $\pm 0.1, \pm 0.05, \pm 0.01$, and \pm 0.005 , showing acoustic and vorticity pulses: $M_{x}=0.5$ and $M_{y}=0.0$ at $(a) t=10,(b) t=40$, (c) $t=50$, and $(d) t=70$, with $\sigma_{m}=\beta=2$ and NPML $=10$. 
(a)

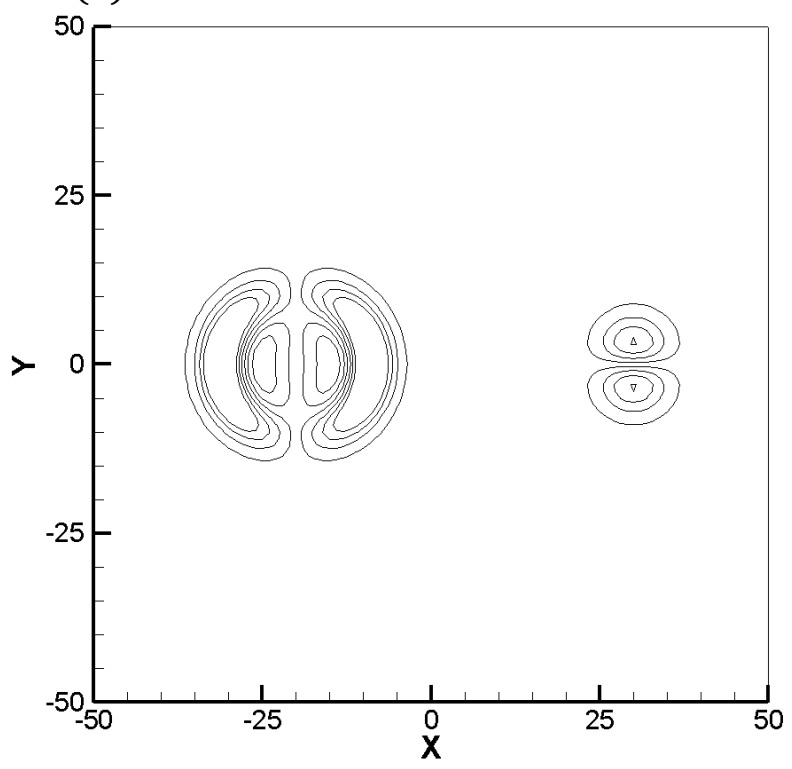

(c)

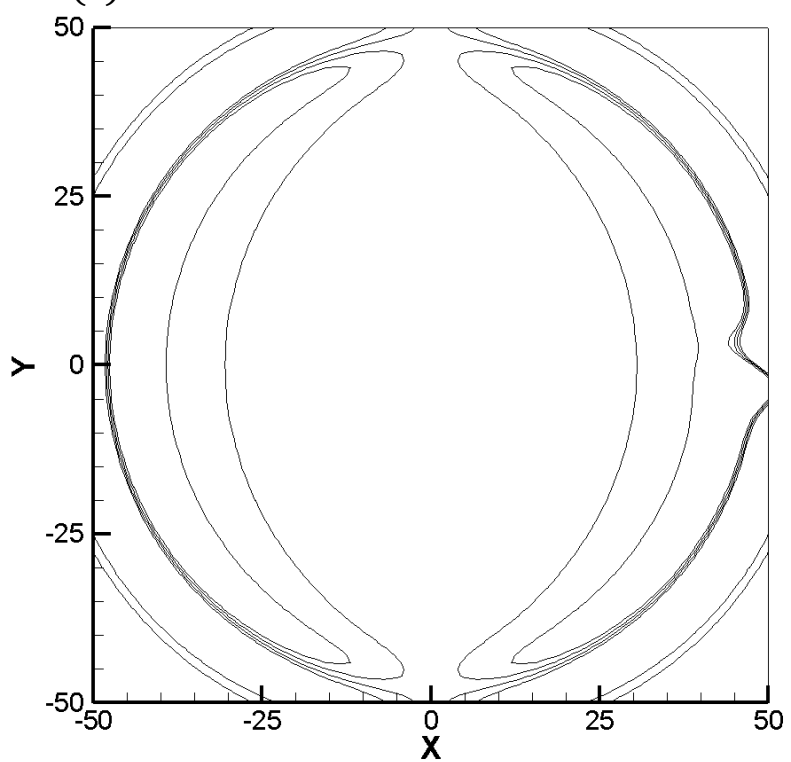

(b)

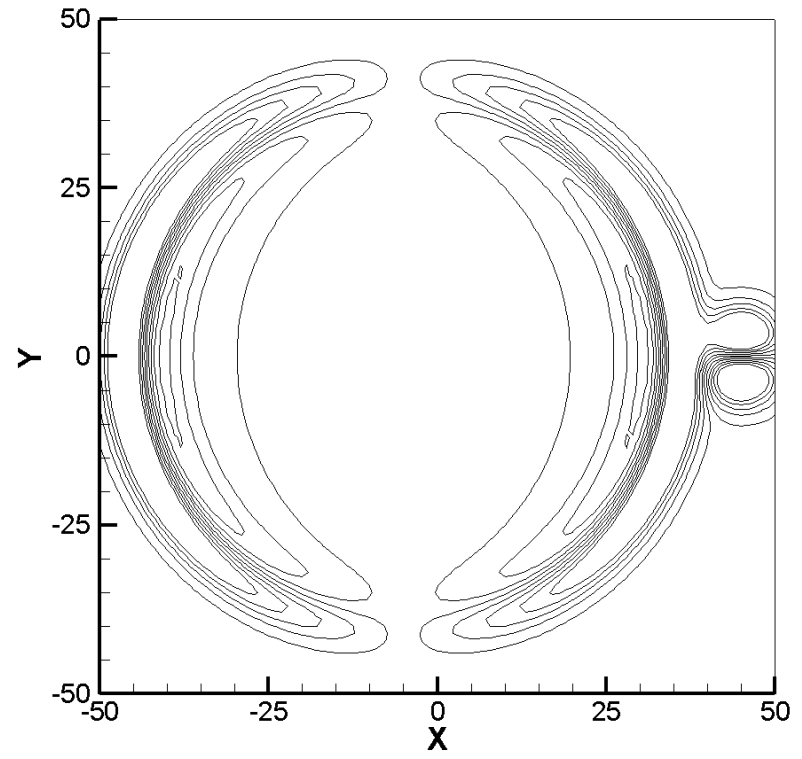

(d)

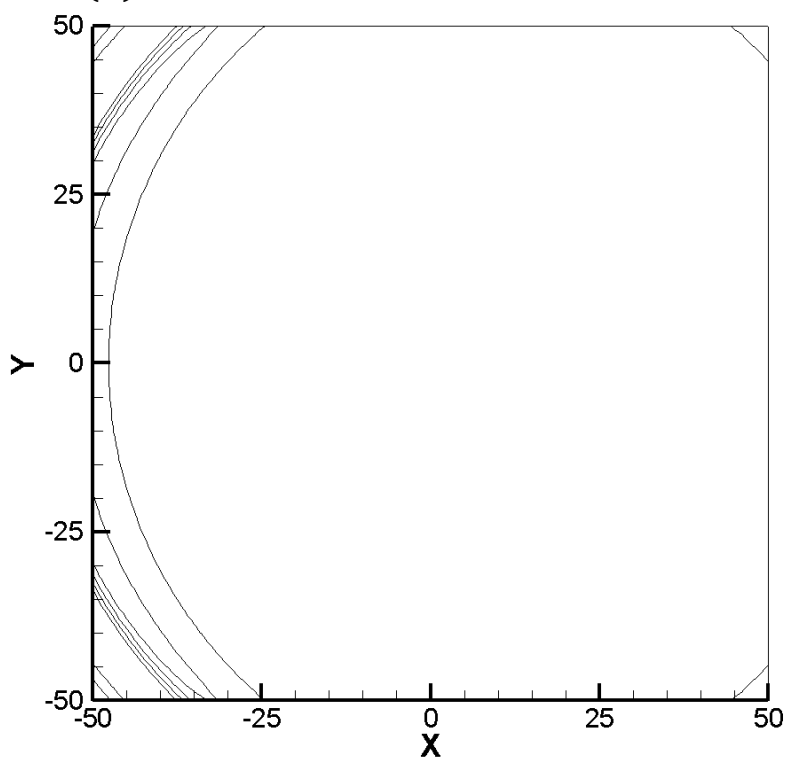

Figure 5.3. Velocity $(u)$ contours at values of $\pm 0.1, \pm 0.05, \pm 0.01$, and \pm 0.02 , showing acoustic and vorticity pulses: $M_{x}=0.5$ and $M_{y}=0.0$ at $(a) t=10$, (b) $t=40$, (c) $t=50$, and (d) $t=70$, with $\sigma_{m}=\beta=2$ and NPML $=10$. 
(a)

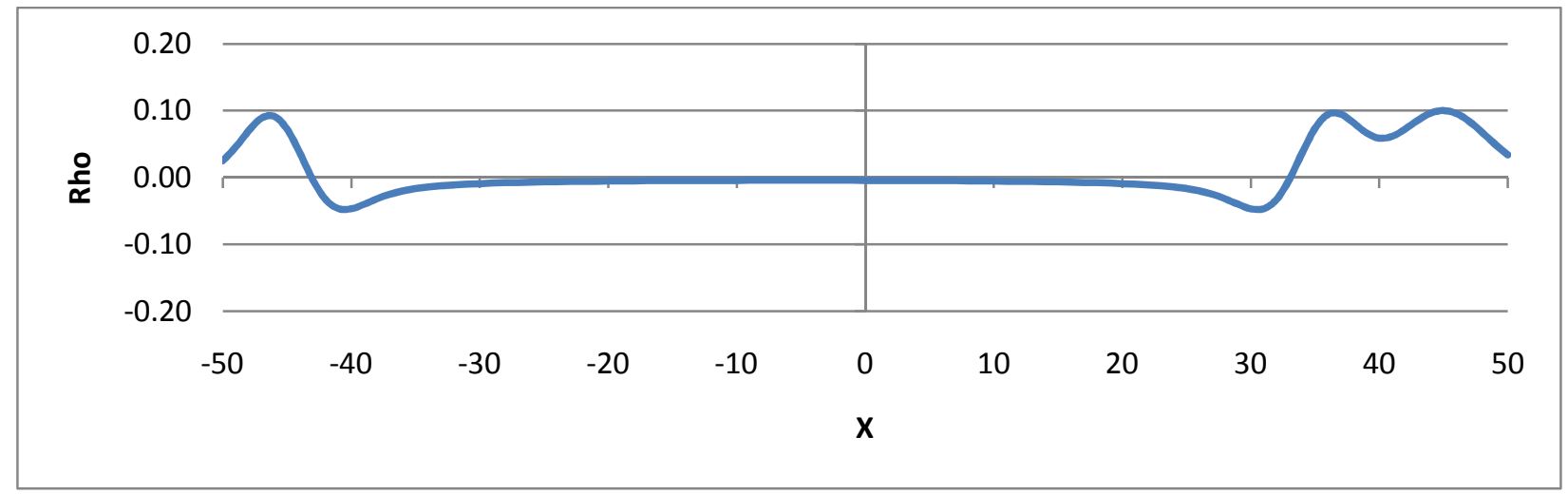

(b)

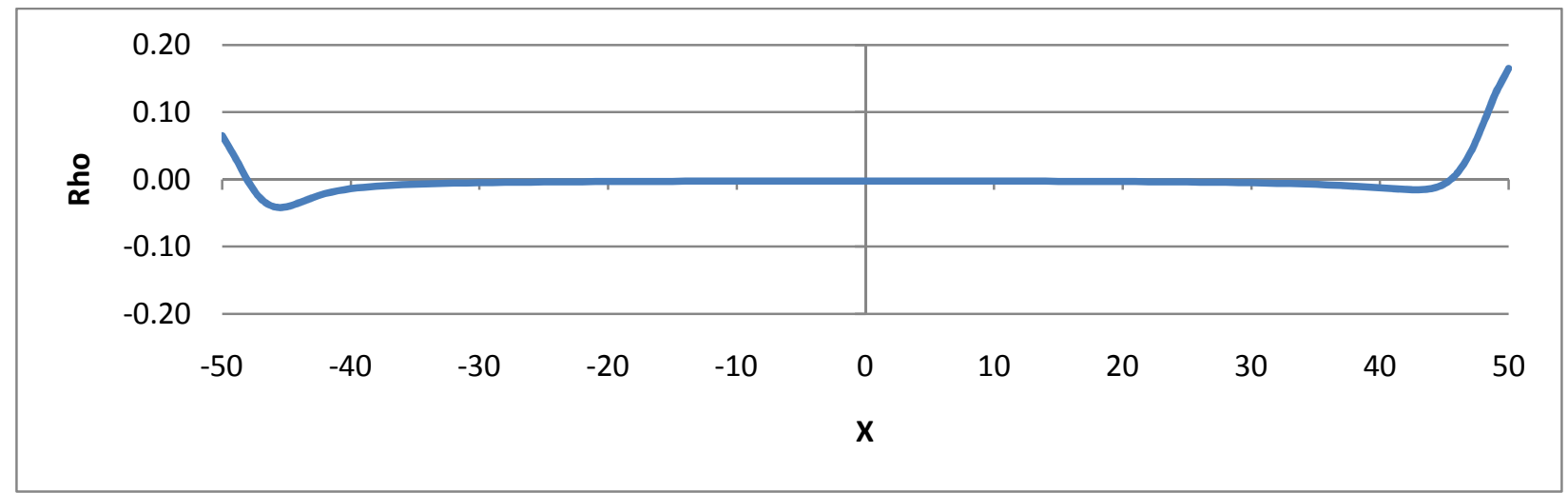

(c)

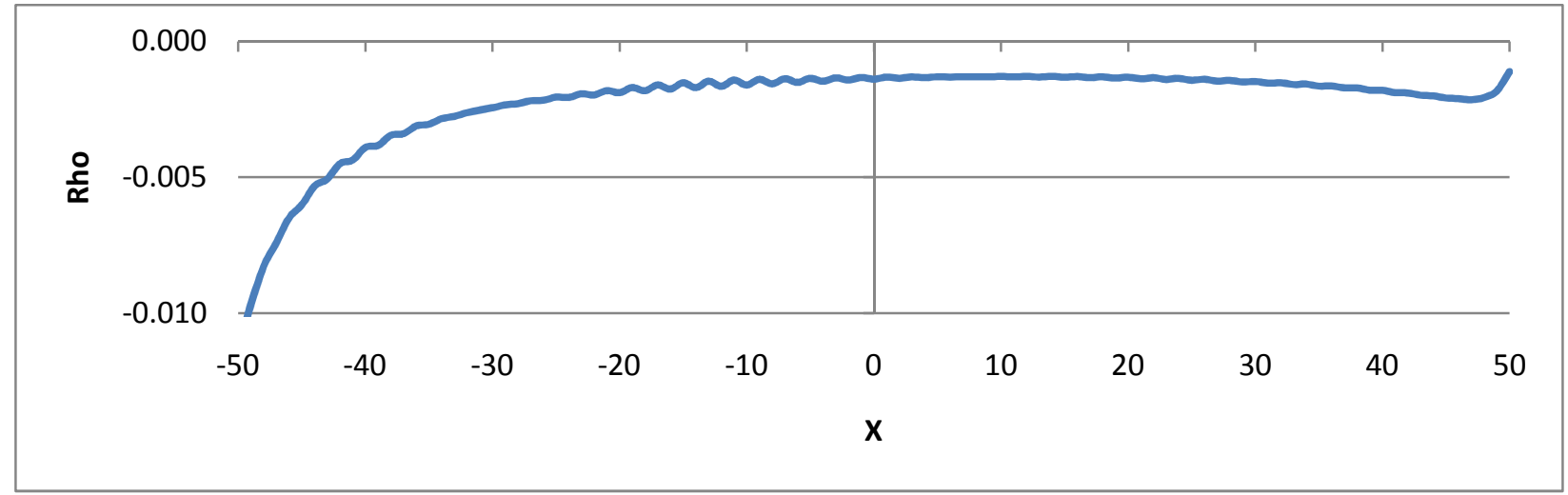

Figure 5.4. Density along line $y=0$ for $M_{x}=0.5$ and $M_{y}=0.0$ at $(a) t=40,(b) t=50$, and $(c) t=70$, with $\sigma_{m}=\beta=2$ and NPML $=10$. 
(a)

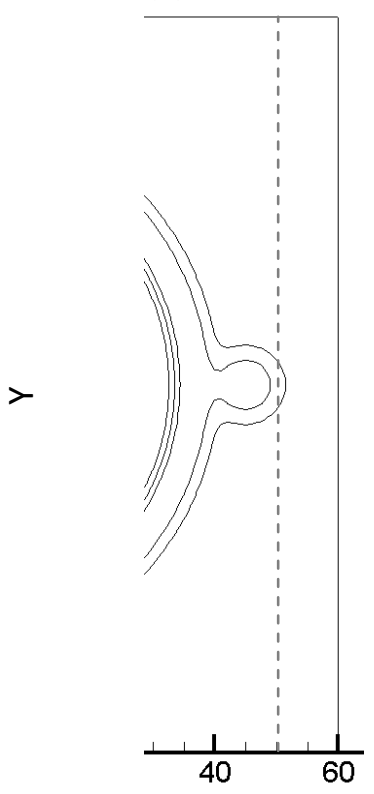

(b)

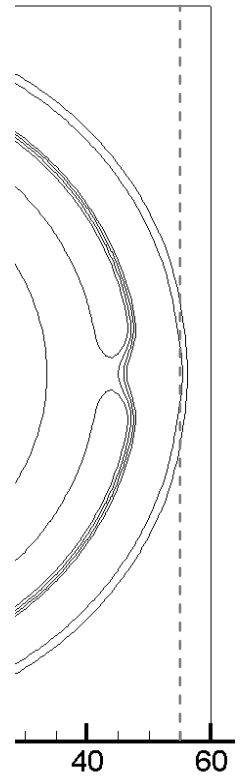

(c)

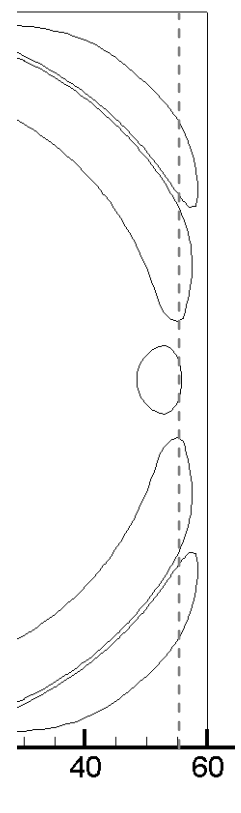

(d)

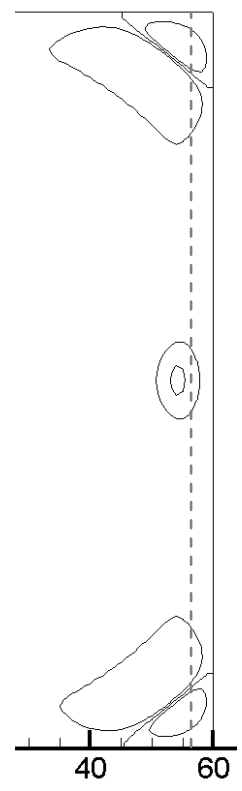

Figure 5.5. Density contours at values of $\pm 0.05, \pm 0.02, \pm 0.01$, and \pm 0.008 , near the right boundary, showing the decaying of waves in the PML domain: $M_{x}=0.5$ and $M_{y}=0.0$ at (a) $t=40,(b) t=50,(c) t=60$, and (d) $t=70$, with $\sigma_{m}=\beta=2$ and NPML $=10$.

\subsection{PML vs. Simple Extrapolation Boundary - A Comparative Evaluation}

The PML formulation, validated in the previous section, showed that it yields reflection-free boundaries and, thus, a very accurate solution. The benefits of this formulation will not be realized until the solution is compared with that of other techniques. For this, a domain of size $[-50,50] \times[-50,50]$ with four open boundaries was considered. The Euler domain was initialized with acoustic, vorticity, and entropy pulses, having amplitudes of $A_{0}, B_{0}$, and $C_{0}$, respectively as follows [12]:

$$
\begin{gathered}
\rho=A_{0} \exp \left[-(\ln 2)\left(\frac{(x+20)^{2}+y^{2}}{16}\right)\right]+C_{0} \exp \left[-(\ln 2)\left(\frac{(x-20)^{2}+y^{2}}{16}\right)\right] \\
p=A_{0} \exp \left[-(\ln 2)\left(\frac{(x+20)^{2}+y^{2}}{16}\right)\right]
\end{gathered}
$$




$$
\begin{gathered}
u=B_{0} y \exp \left[-(\ln 2)\left(\frac{(x-20)^{2}+y^{2}}{16}\right)\right] \\
v=B_{0}(x-20) \exp \left[-(\ln 2)\left(\frac{(x-20)^{2}+y^{2}}{16}\right)\right]
\end{gathered}
$$

The propagation of these pulses was simulated with a mean flow of Mach number $M=0.9$. The amplitude of these pulses was $A_{0}=B_{0}=C_{0}=1$. A uniform grid of $\Delta x=\Delta y=1$ with a time step $\Delta t=0.5$ was used. Both Euler and PML equations were solved numerically by an implicit sixth-order five-point central difference scheme. The time integration was carried out by a fourth-order Runge-Kutta scheme. No numerical damping was used in all the computations reported here. For the case of zero-order extrapolation, a simple extrapolation technique was applied on the outermost boundaries of the computational domain.

In the case of PML, a layer of ten grid points, i.e., $D=10 \Delta x$, was appended to the Euler's domain in all four directions, and simple extrapolation was used on the PML boundaries. Further, the solution obtained from the PML case was compared with a reference solution obtained by computing the flow with the same spatial and temporal resolution but in a much larger domain so that the solution is not affected by the boundary conditions. Since a wide stencil was used in the finite difference scheme, the absorption coefficients were varied gradually inside the PML domain, as given by equation (3.11). Values of $\sigma_{x} \Delta x=2$, where $\Delta x$ is the grid size, and $\beta=10$ were used.

Figure 5.6 shows the velocity $(v)$ contour at $t=50$ for all the three cases. The quality of the solution is clearly evident from the contour plots. It can be observed from Figure 5.6(b) that the domain without PML was affected by the reflecting waves at the 
boundaries, which spoiled the solution. On the other hand, the domain with PML boundaries had an accurate solution, which can be seen in Figure 5.6(c). It can also be observed that the solution obtained with PML boundaries is the same as that of the reference solution, shown in Figure 5.6(a). Thus, the application of PML with a smaller domain is very accurate and it is very efficient as the time required to solve the problem is reduced to a greater extent. Figure 5.6(d) shows the solution in both Euler and PML domains. The damping quality of the PML technique is clearly visible. However, the solution in the PML domain has no physical significance unless it causes any reflections back into the Euler domain.

Figure 5.7 shows the velocity $(v)$ distribution along the $y=0$ line. The solution obtained using PML boundary conditions match exactly with the reference solution, whereas the extrapolation solution did not. Figure 5.8 shows a plot of the time history of $v$ at a location on the interface of the Euler and a PML domain, $(x, y)=(50,5)$. The oscillations of the extrapolation solution can be clearly seen here. Also plotted in Figure 5.9 is the difference in the solutions obtained from simple extrapolation and PML boundary conditions as a function of time. 
(a)

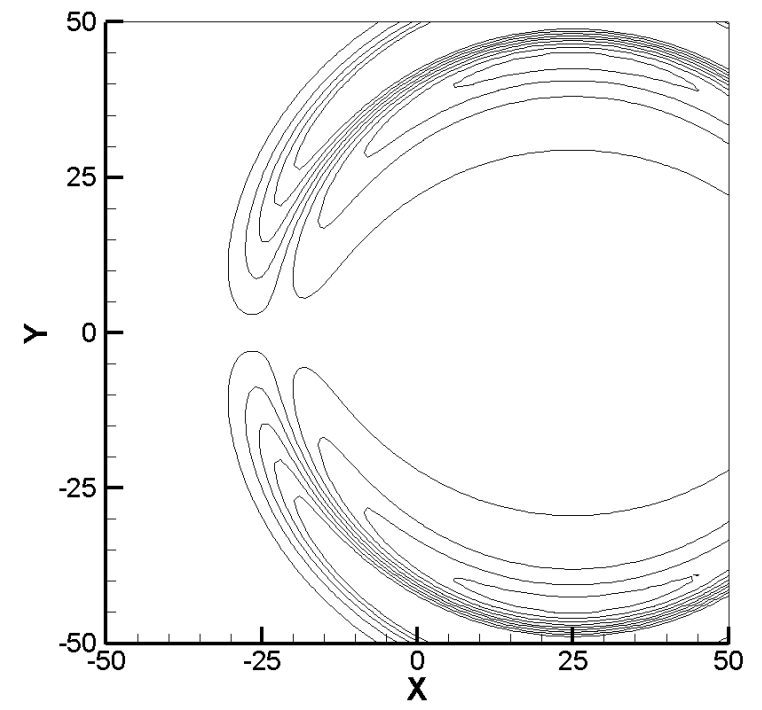

(c)

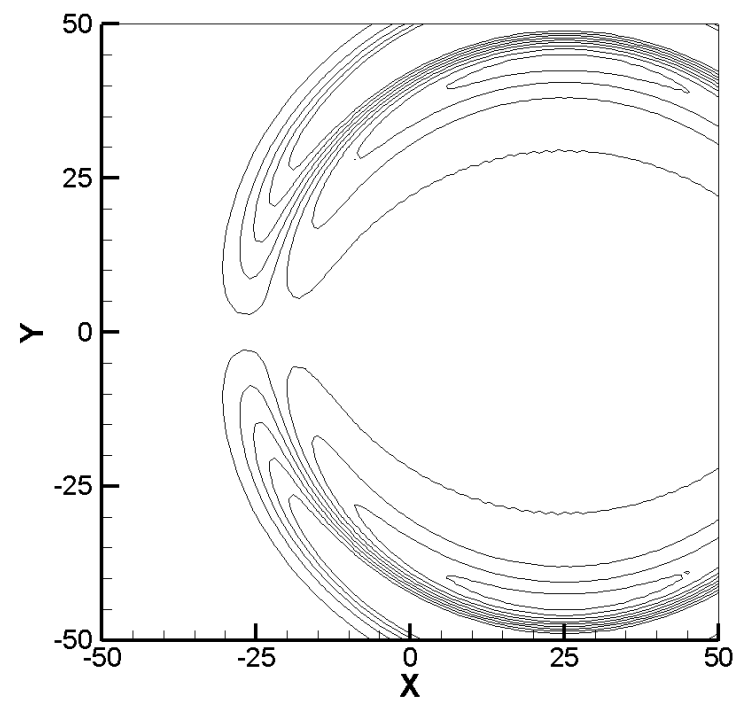

(b)

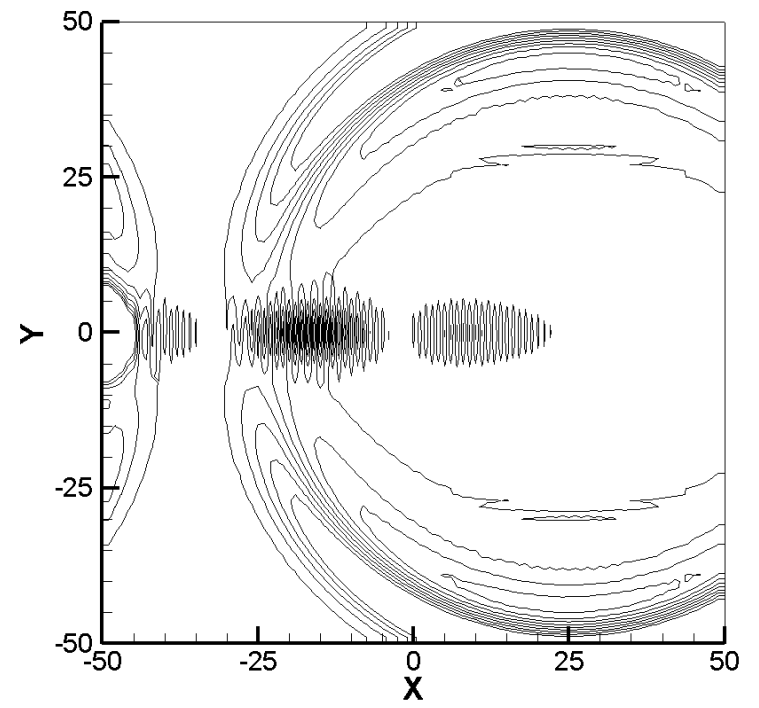

(d)

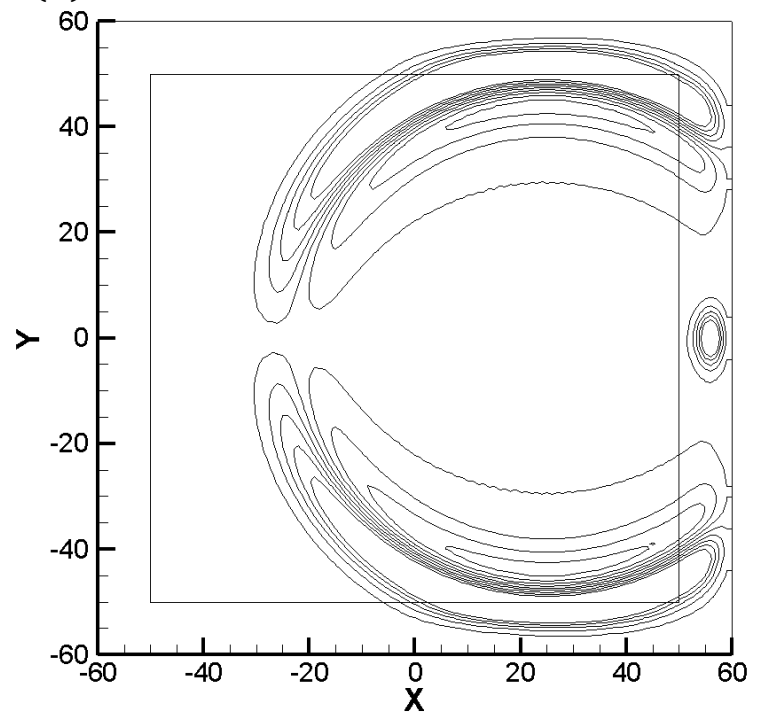

Figure 5.6. Velocity $(v)$ contours at values of $\pm 0.05: M_{x}=0.9$ and $M_{y}=0.0$ at $t=50$ :

(a) reference solution, (b) zero-order extrapolation, (c) PML solution with ten grid points, and (d) solution in interior domain and PML domain. 


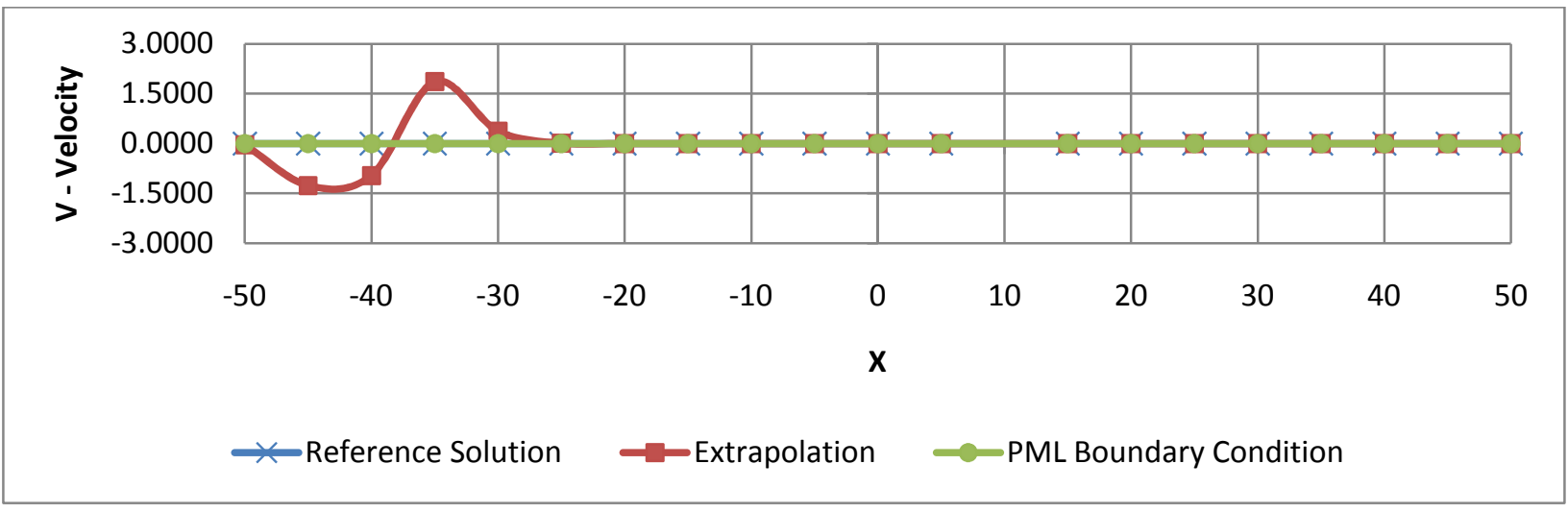

Figure 5.7. Velocity $(v)$ distribution along $y=0$ line with $M_{x}=0.9$ and $M_{y}=0.0$ at $t=50$.

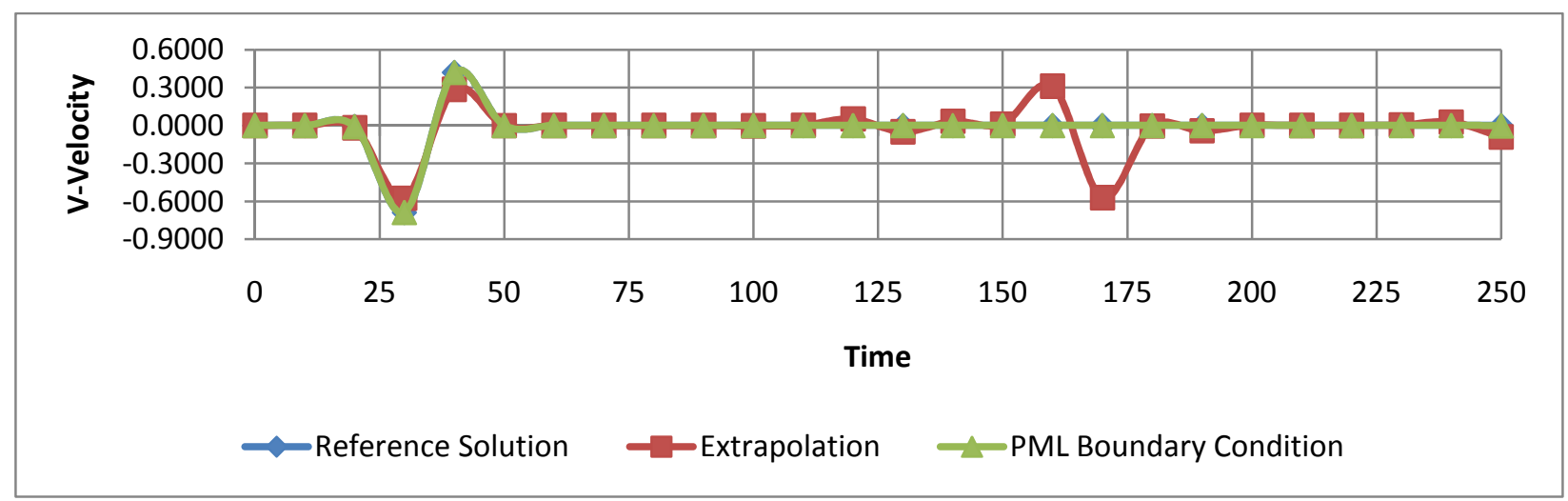

Figure 5.8. Velocity $(v)$ component as a function of time at point $(x, y)=(50,5)$.

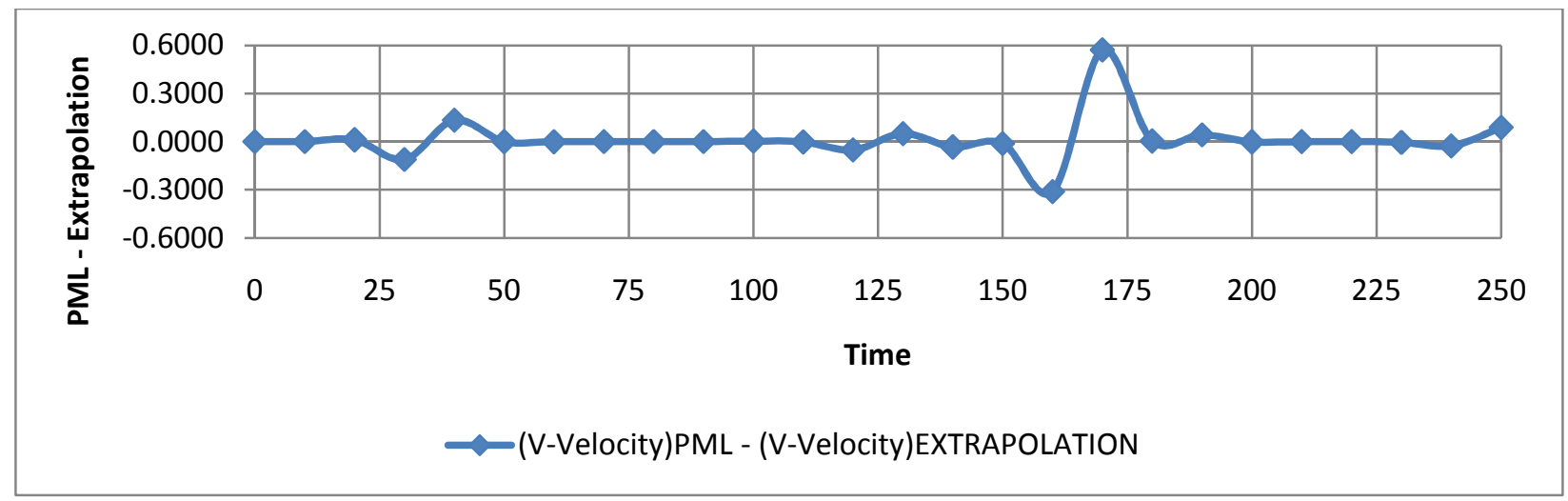

Figure 5.9. Difference between PML and simple extrapolation solutions as a function of time at point $(x, y)=(50,5)$. 


\subsection{Validation of PML Equations in Generalized Coordinates}

The importance of PML equations in generalized coordinates was discussed in section 3.4. In order to validate PML equations in the generalized coordinates, PML boundary conditions were implemented over a non-uniform grid. The uniform and nonuniform grid structures and their respective physical and PML domains are shown in Figure 5.10. The details of the geometrical definitions of both uniform and non-uniform grid structures have already been discussed in section 4.1. A PML domain of 25 grid points has been used around the four sides of the physical domain in both grid structures. In this section, two cases-parallel flow and oblique mean flow-will be considered to determine how the PML technique behaves in different grid structures.

\subsubsection{Parallel Flow Case}

Two cases each for uniform and non-uniform grids were considered. The first one was a pressure pulse, as given by Tam and Auriault [26].

$$
\begin{gathered}
p=\rho=\exp \left[(-\ln 2)\left(\frac{\left(x-x_{a}\right)^{2}+\left(y-y_{a}\right)^{2}}{9}\right)\right] \\
u=v=0
\end{gathered}
$$

The second case is taken from $\mathrm{Hu}[10]$. These conditions are same as of the one used in section 5.1 for validation, where the amplitude of these pulses are $A_{0}=1, B_{0}=0.05$, and $C_{0}=0.1$. The initial conditions are given by;

$$
\begin{gathered}
\rho=\exp \left[-(\ln 2)\left(\frac{\left(x-x_{a}\right)^{2}+\left(y-y_{a}\right)^{2}}{9}\right)\right]+0.1 \exp \left[-(\ln 2)\left(\frac{\left(x-x_{b}\right)^{2}+\left(y-y_{b}\right)^{2}}{16}\right)\right] \\
p=\exp \left[-(\ln 2)\left(\frac{\left(x-x_{a}\right)^{2}+\left(y-y_{b}\right)^{2}}{9}\right)\right]
\end{gathered}
$$




$$
\begin{aligned}
& u=0.05\left(y-y_{b}\right) \exp \left[-(\ln 2)\left(\frac{\left(x-x_{b}\right)^{2}+\left(y-y_{b}\right)^{2}}{16}\right)\right] \\
& v=-0.05\left(x-x_{b}\right) \exp \left[-(\ln 2)\left(\frac{\left(x-x_{b}\right)^{2}+\left(y-y_{b}\right)^{2}}{16}\right)\right]
\end{aligned}
$$

These equations include an acoustic pulse centered at $\left(x_{a}, y_{a}\right)$ and a vorticity and an entropy pulse, both centered at $\left(x_{b}, y_{b}\right)$. For the parallel flow case, the mean flow is in the direction of the $x$-axis with $M=0.5$. To demonstrate the application and validity of PML equations in generalized coordinates, comparisons were made between the results computed over a uniform grid and a non-uniform grid. The initial center of the acoustic pulse was $\left(x_{a}, y_{a}\right)=(-12.5,0)$ and that of the vorticity and entropy waves was $\left(x_{b}, y_{b}\right)=(12.5,0)$. The value of $\sigma_{m}=\beta=2$ was selected for the present calculations.

The density contours of the pressure pulse case are shown in Figure 5.11 in which the transmitted waves leaving the physical domain without causing any reflections is seen. Figures 5.12 and 5.13 show the density along the line $x=0$ at $t=30$ and $x=-24$ at $t=80$. It can be seen that the solution obtained using the uniform grid as well as the non-uniform grid structures compare well. Figure 5.14 show the velocity $(u)$ contours of the pressure pulse case for both uniform and non-uniform grids. Figures 5.15 and 5.16 present the velocity $(u)$ along the line $x=0$ at $t=30$ and $x=-24$ at $t=80$. It is clearly seen that the solution from both grid structures match well.

The density contours of the acoustic and vorticity pulse case are shown in Figure 5.17. The comparison of density along line $x=0$ is presented in Figures 5.18 and 5.19 
at $t=10$ and $t=40$ respectively. In the same way, the velocity $(u)$ contours of this acoustic and vorticity pulse case are shown in Figure 5.20. The comparison of velocity (u) along line $x=-20$ is presented in Figure 5.21 and along $x=-15$ in Figure 5.22 for $t=40$ and $t=70$ respectively. The departure of the transmitted waves without any reflections and the identicalness of the solution from the different grid structures are quite evident. Thus, it was verified that non-rectangular domains can be modified into rectangular domains using generalized coordinates, and PML formulation remains valid in the modified domains. 
(a)

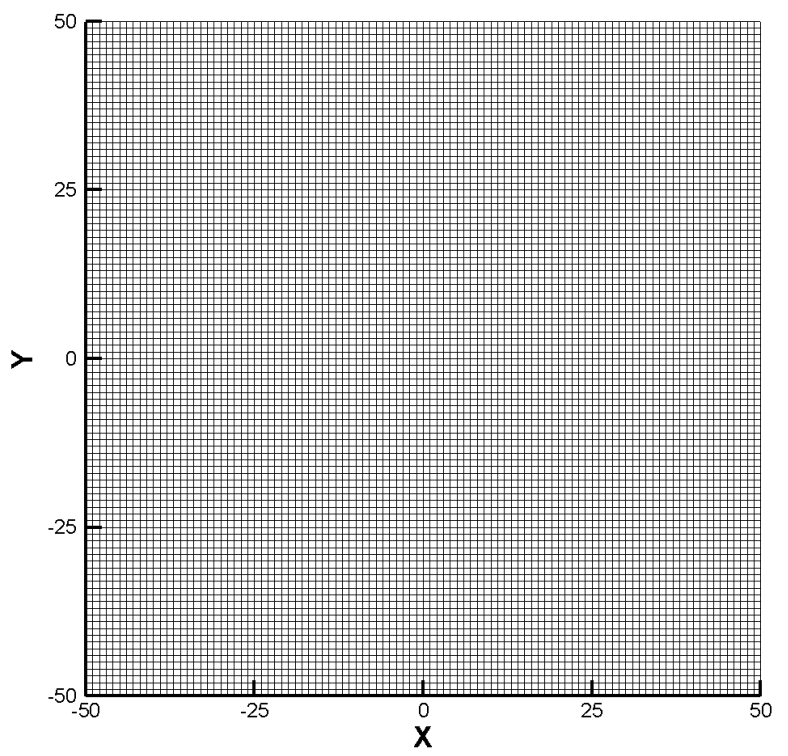

(c)

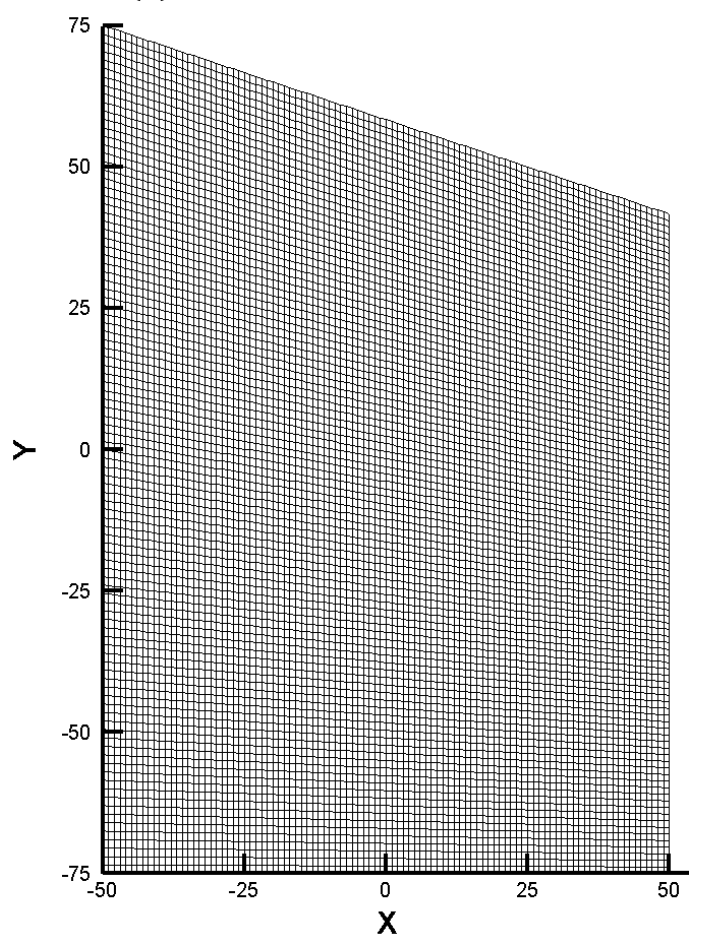

(b)

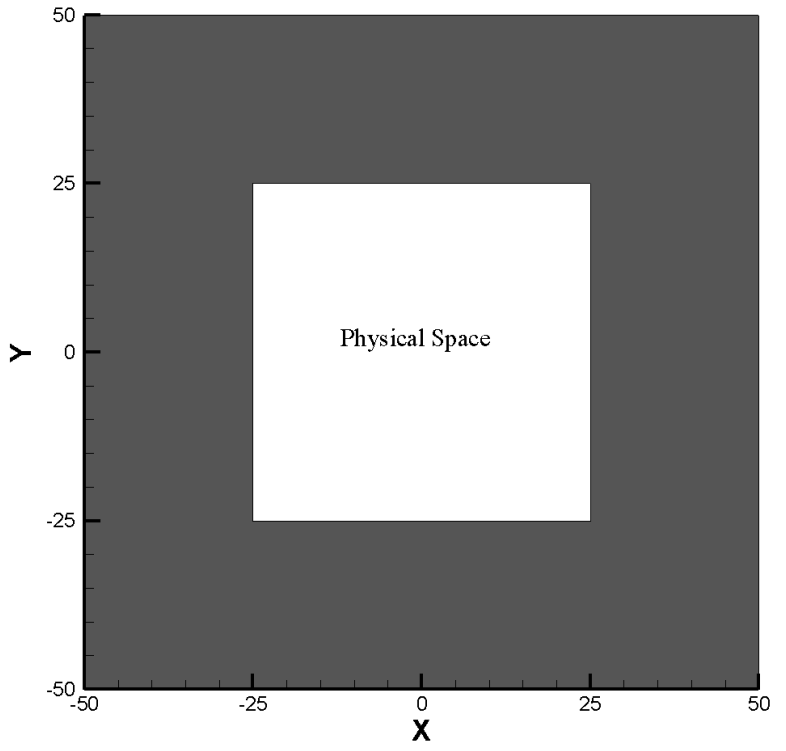

(d)

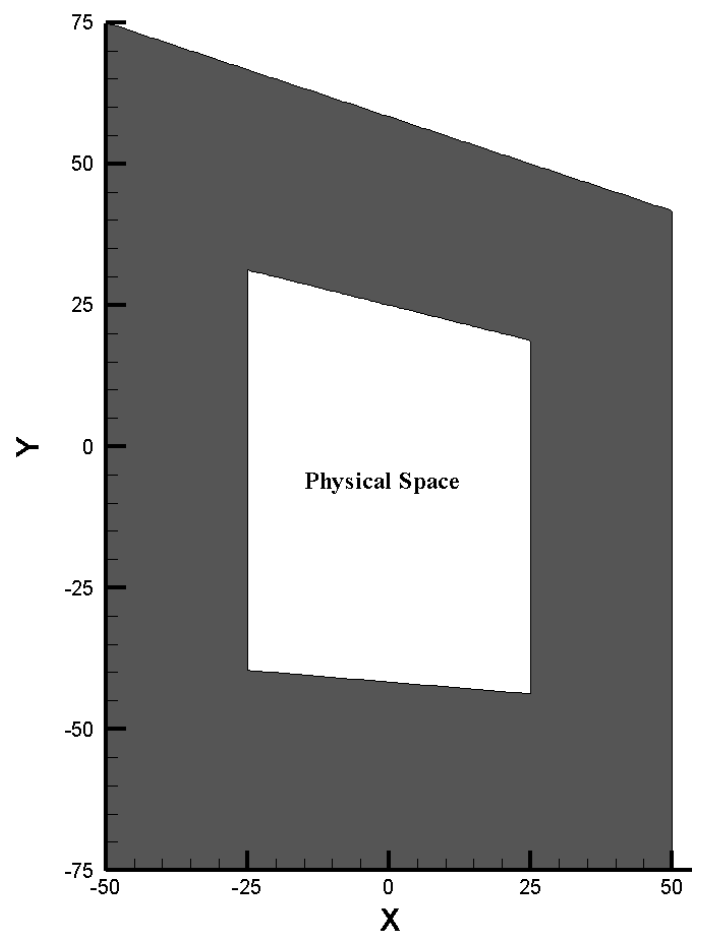

Figure 5.10. Uniform grid: (a) grid structure, (b) PML domain and physical space. Non-uniform grid: (c) grid structure, (d) PML domain and physical space. Number of layers $=25$. 
(a)

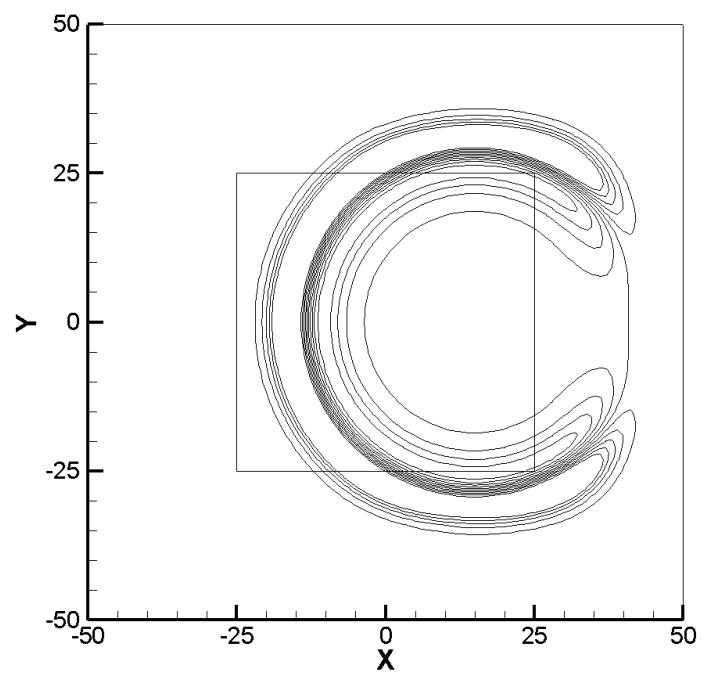

(b)

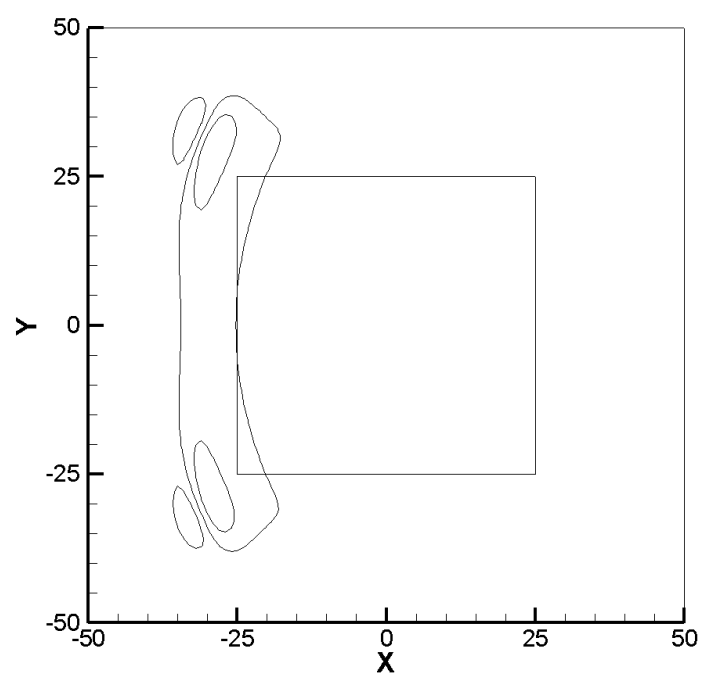

(a)

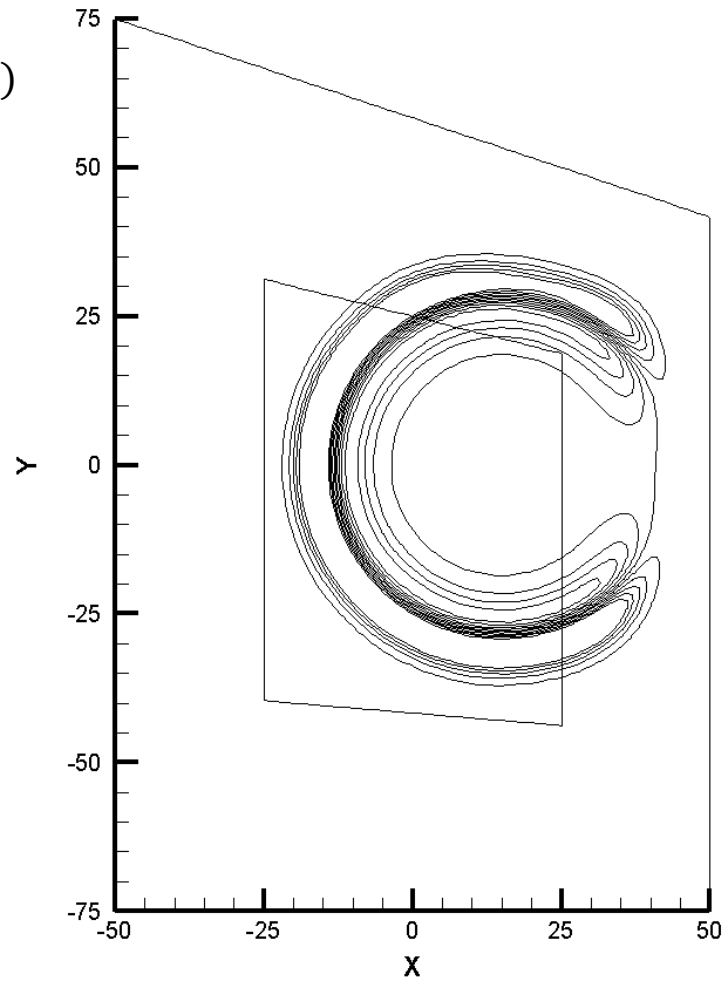

(b)

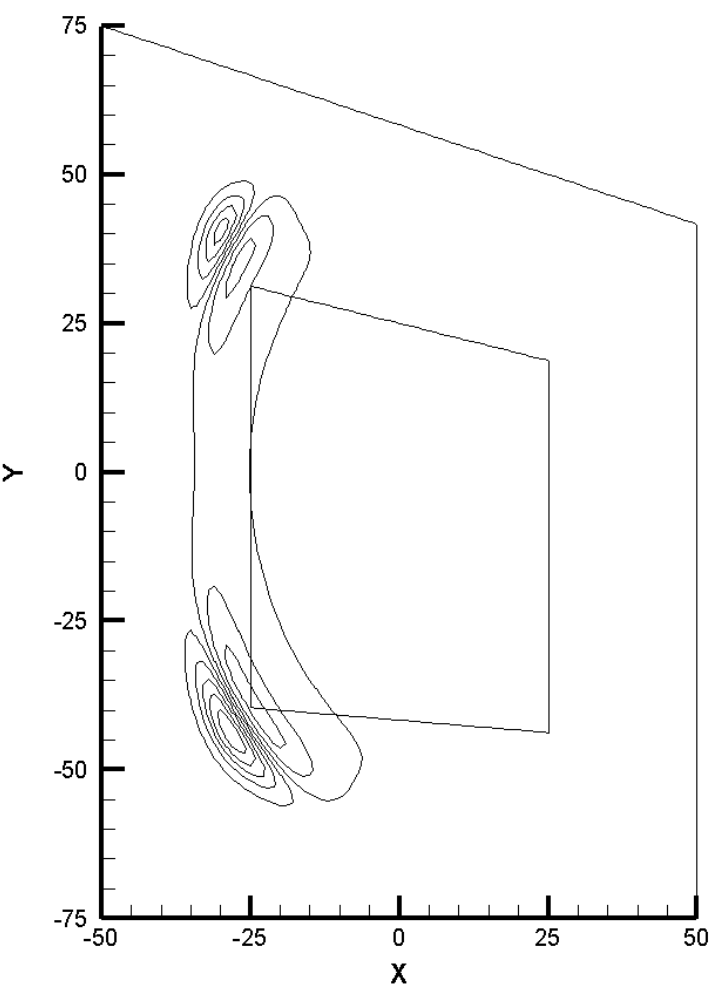

Figure 5.11. Density contours at values of \pm 0.1 and \pm 0.05 with uniform and non-uniform grids, showing acoustic pulse: $M_{x}=0.5$ and $M_{y}=0.0$ at $(a) t=30$ and $(b) t=80$. 


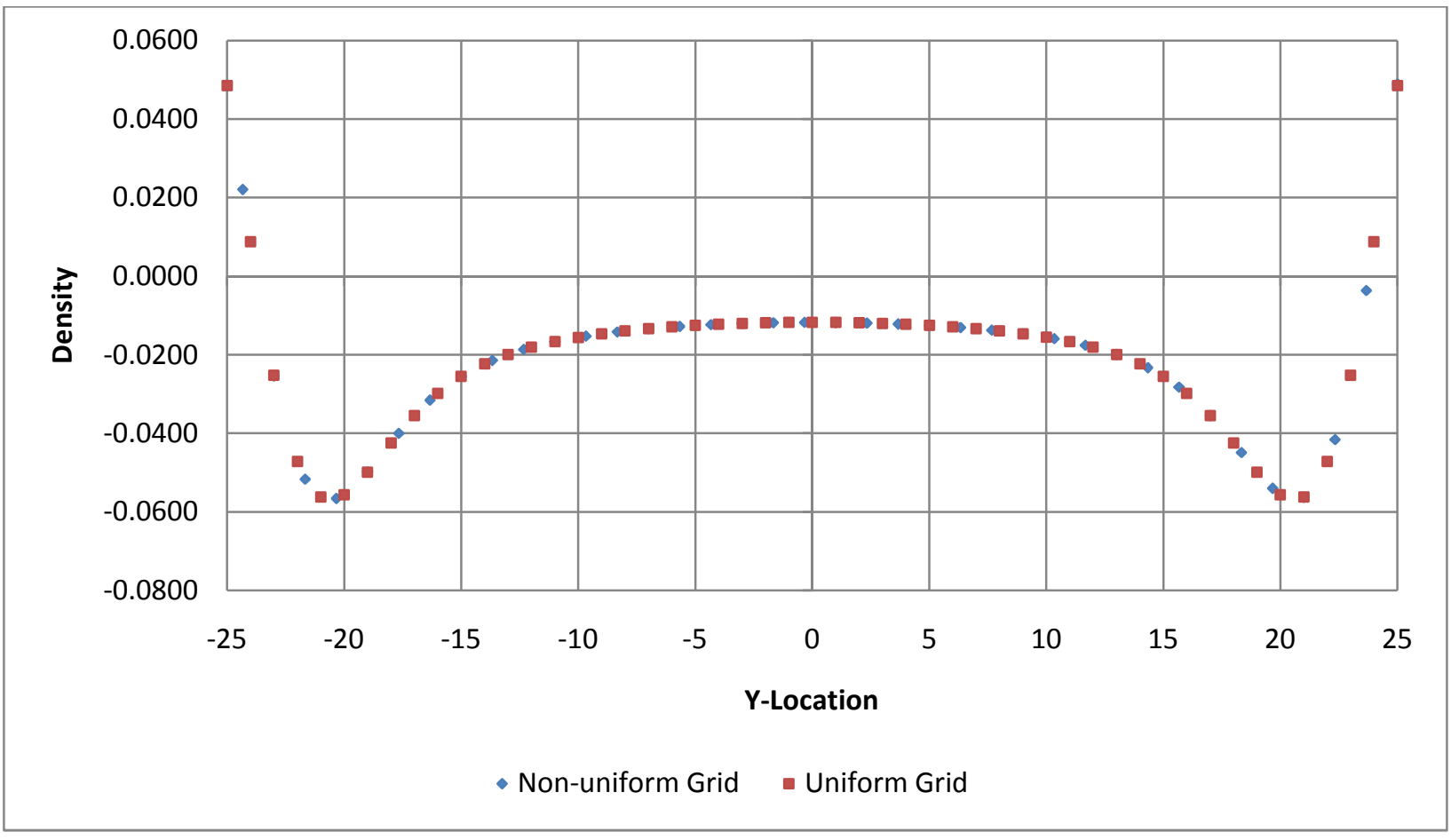

Figure 5.12. Density along line $x=0$ with $M_{x}=0.5$ and $M_{y}=0.0$ at $t=30$.

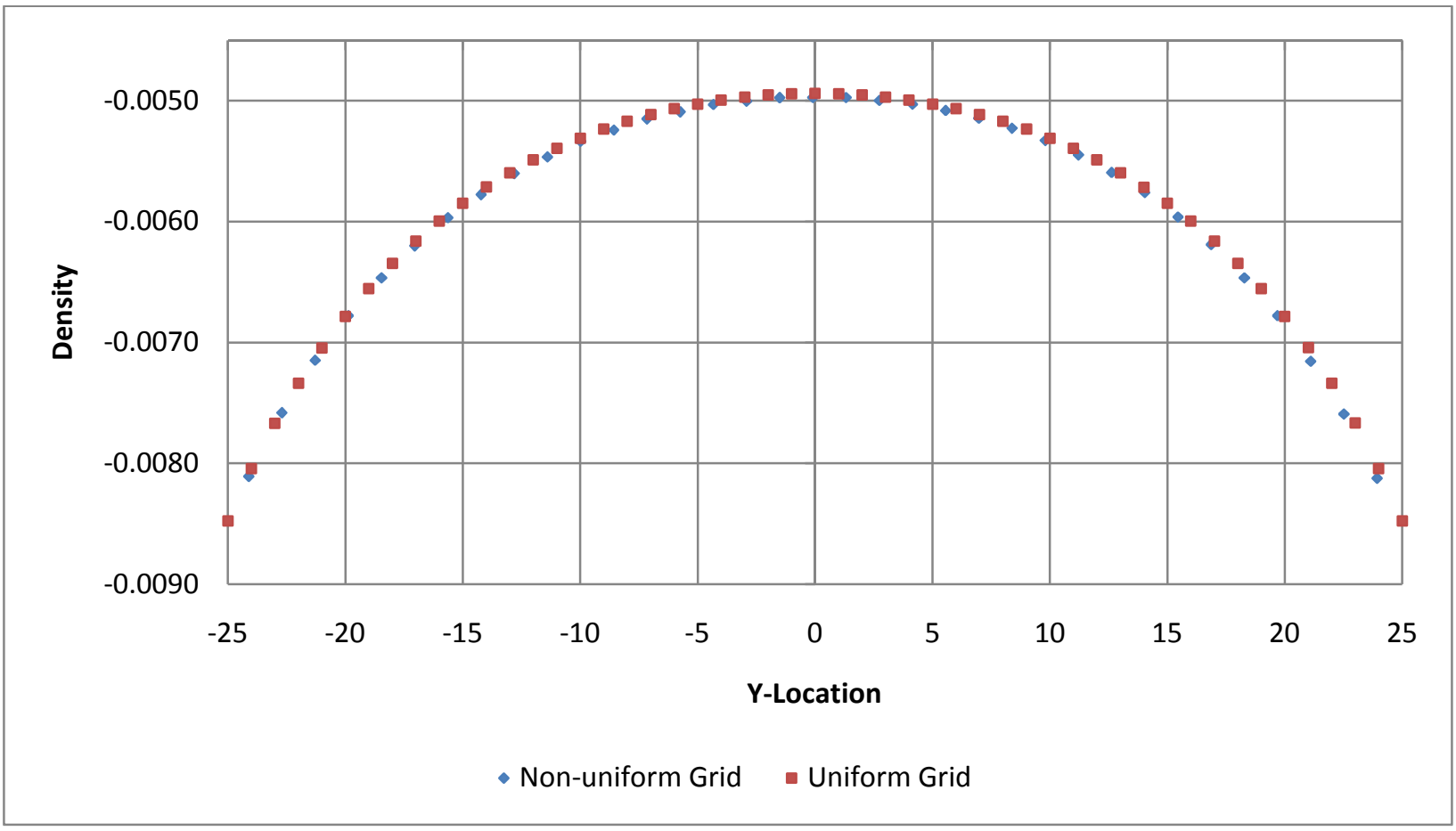

Figure 5.13. Density along line $x=-24$ with $M_{x}=0.5$ and $M_{y}=0.0$ at $t=80$. 
(a)

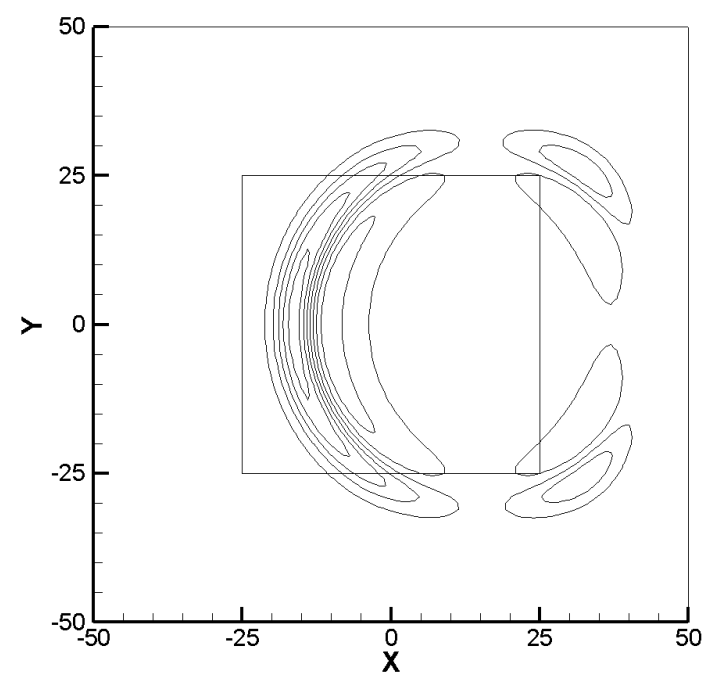

(b)

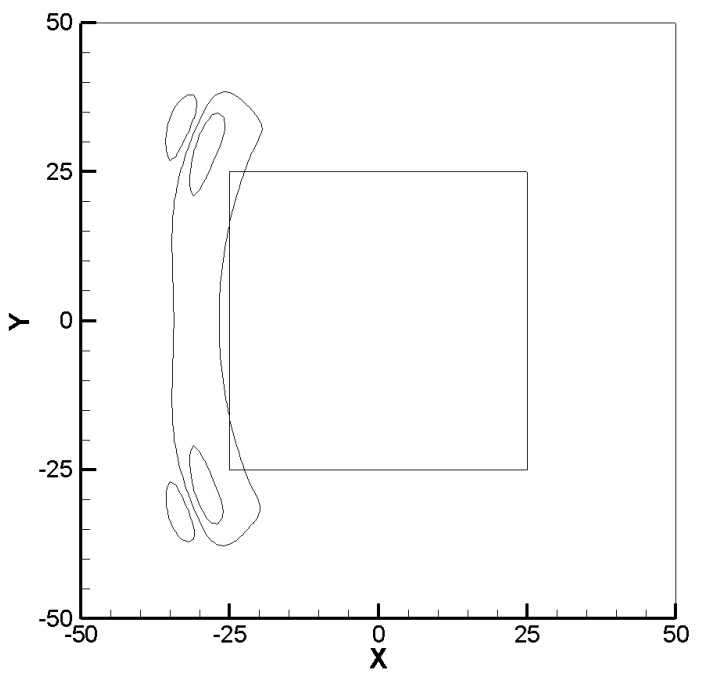

(a)

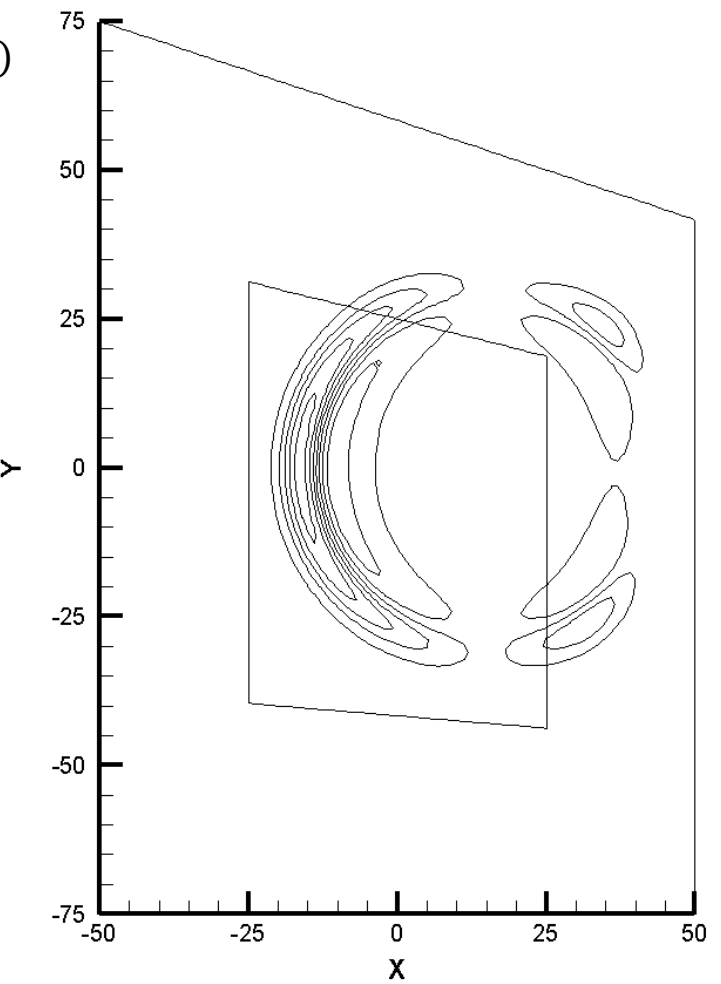

(b)

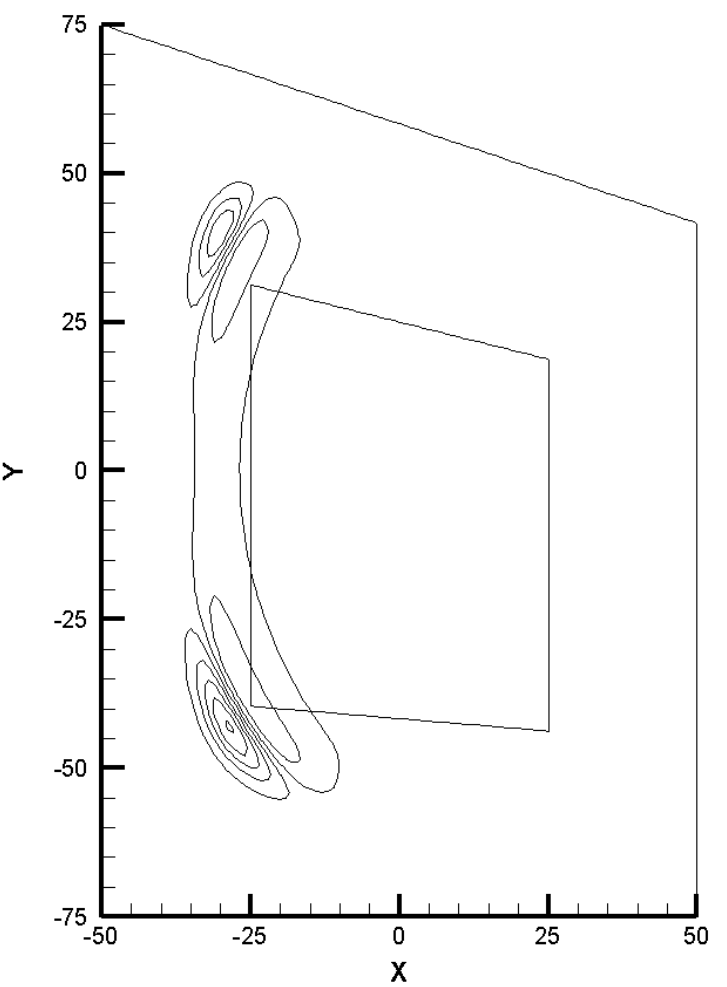

Figure 5.14. Velocity $(u)$ contours at values of \pm 0.1 and \pm 0.05 with uniform and nonuniform grids, showing acoustic pulse: $M_{x}=0.5$ and $M_{y}=0.0$ at $(a) t=30$ and (b) $t=80$ 


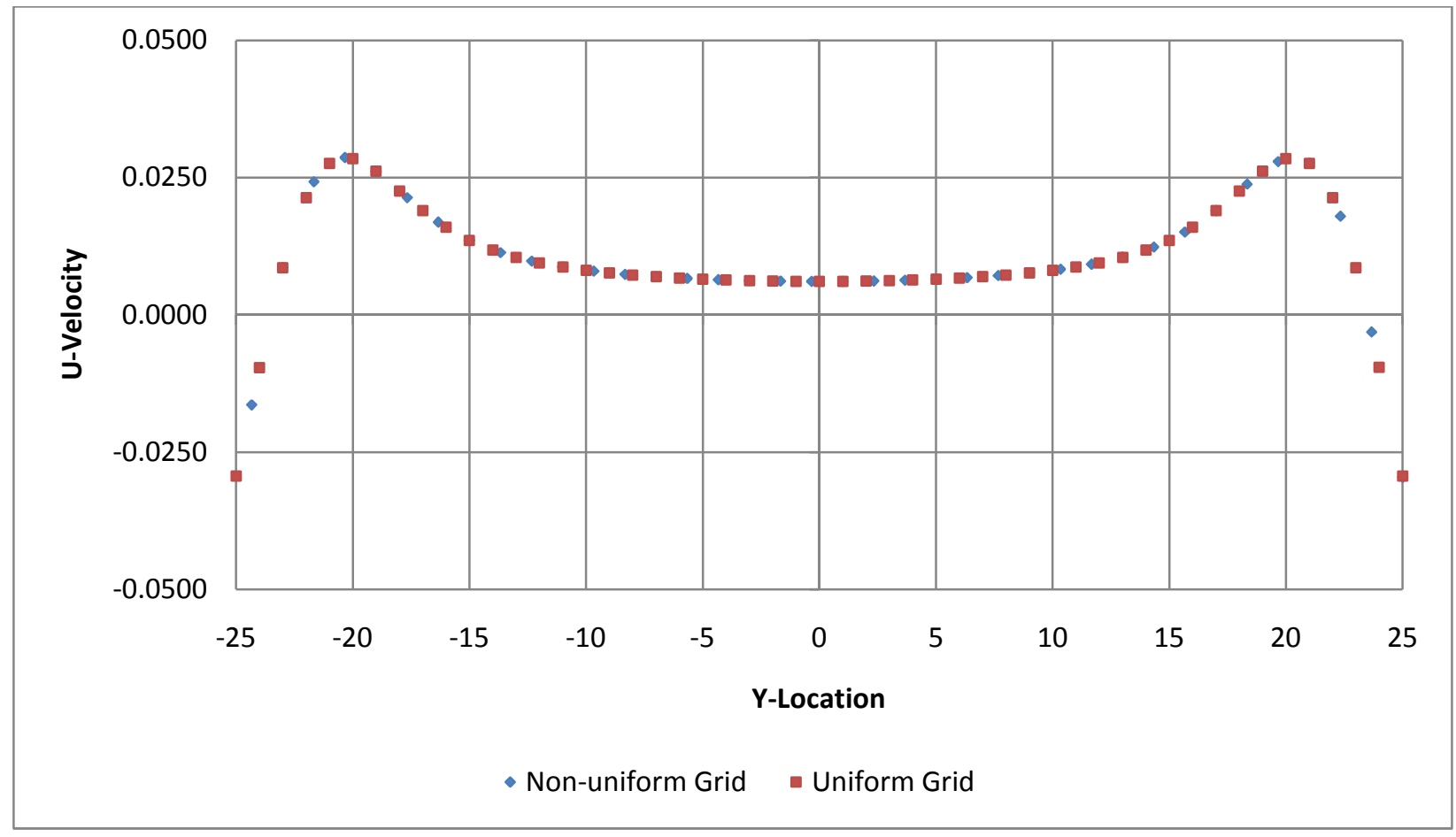

Figure 5.15. Velocity $(u)$ component along line $x=0$ with $M_{x}=0.5$ and $M_{y}=0.0$ at $t=30$

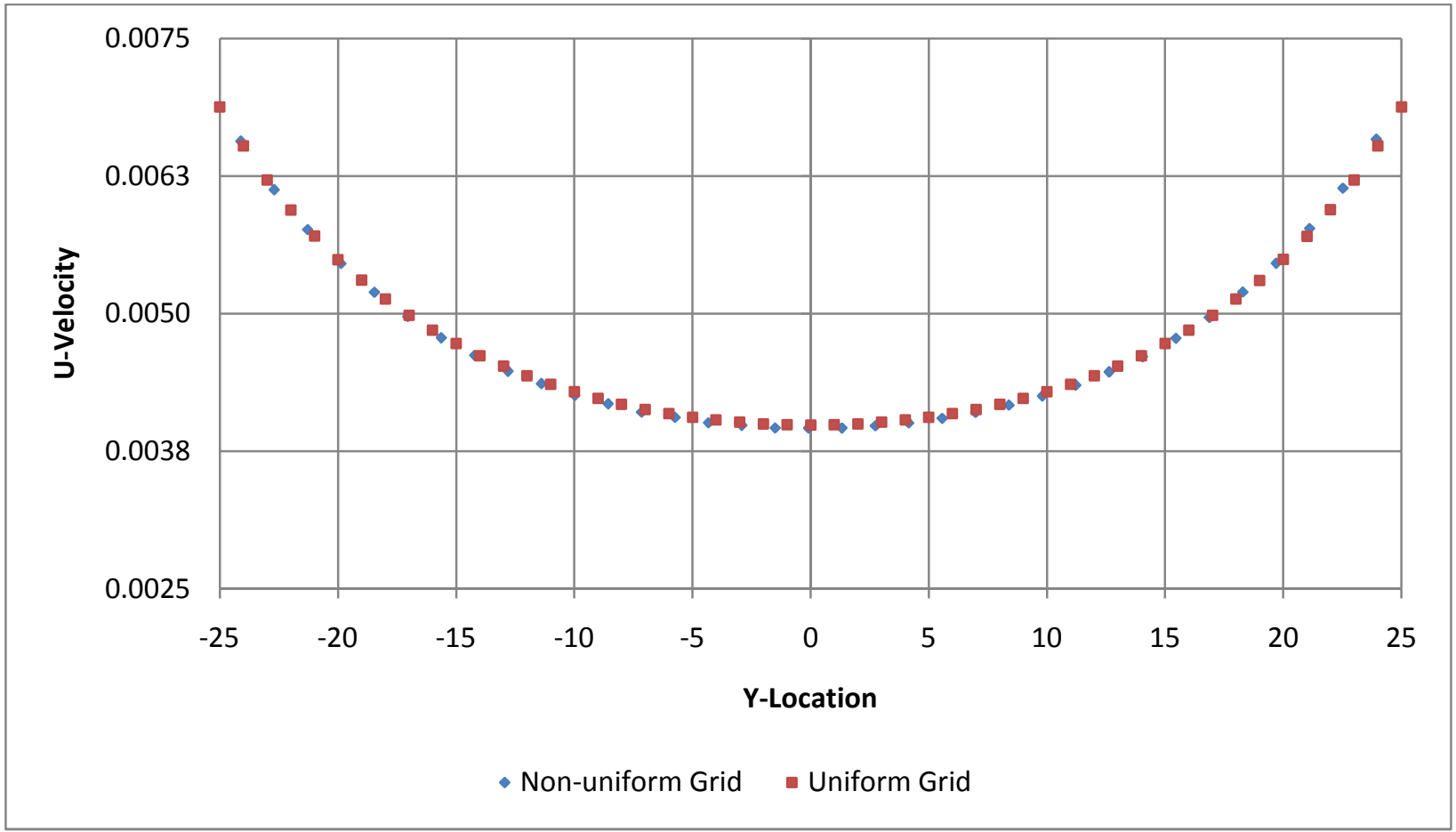

Figure 5.16. Velocity $(u)$ component along line $x=-24$ with $M_{x}=0.5$ and $M_{y}=0.0$ at $t=80$ 
(a)

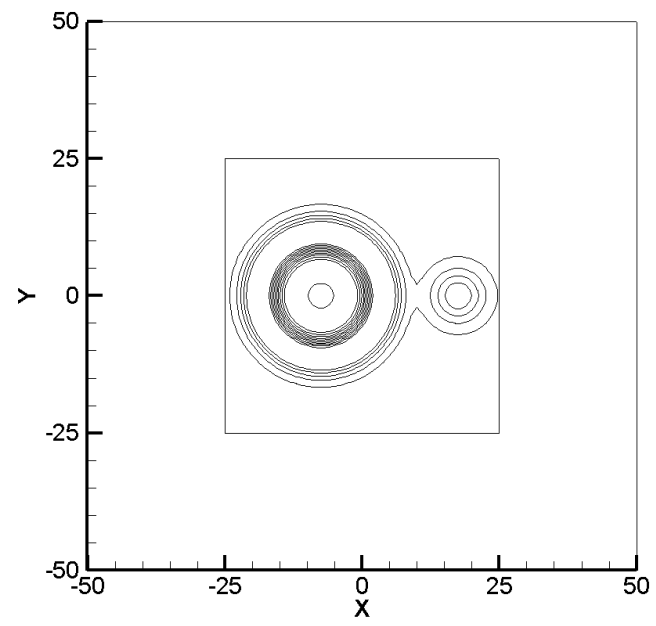

(b)

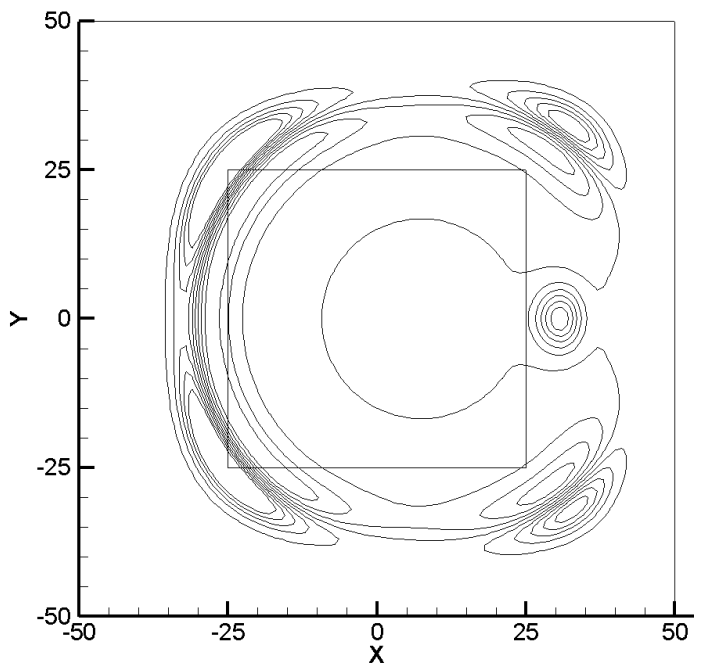

(a)

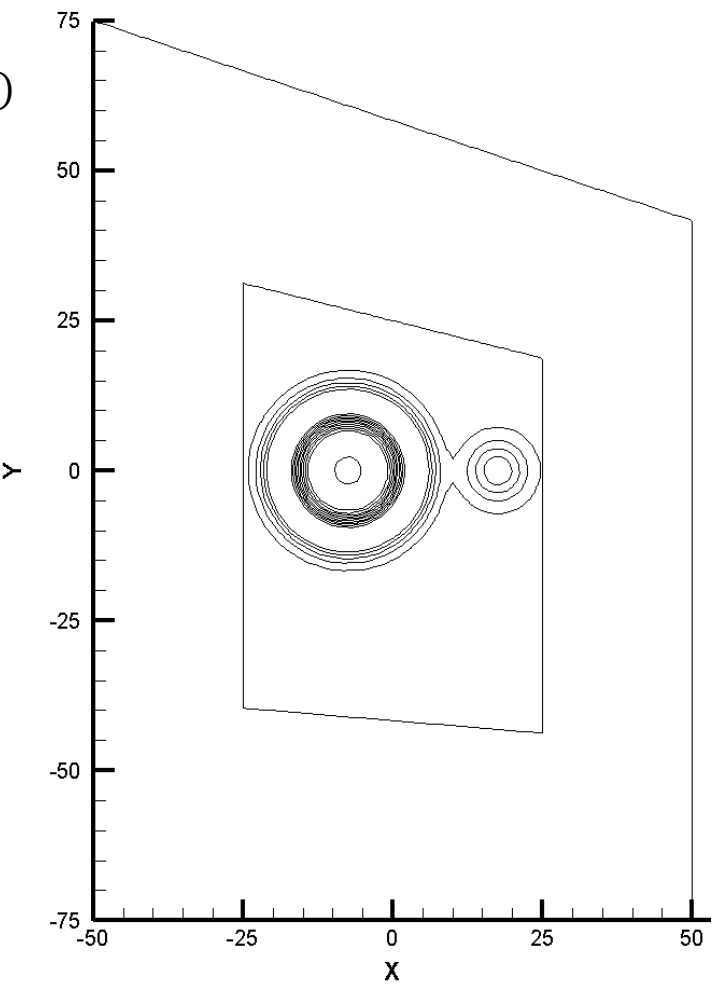

(b)

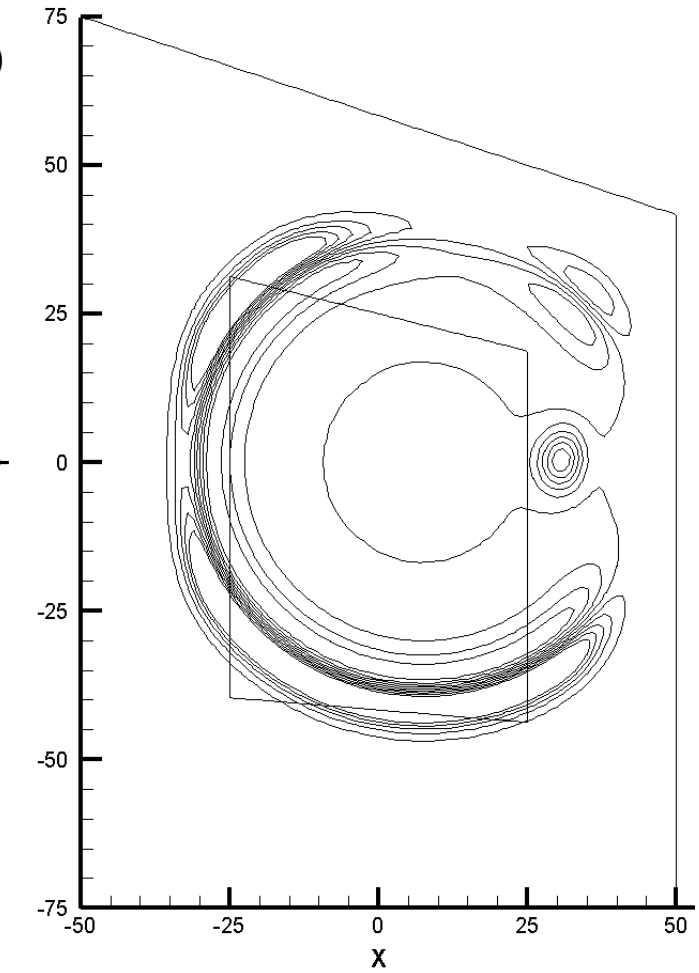

Figure 5.17. Density contours at values of \pm 0.1 and \pm 0.05 with uniform and non-uniform grids, showing acoustic and vorticity pulses: $M_{x}=0.5$ and $M_{y}=0.0$ at $(a) t=10$ and (b) $t=40$. 


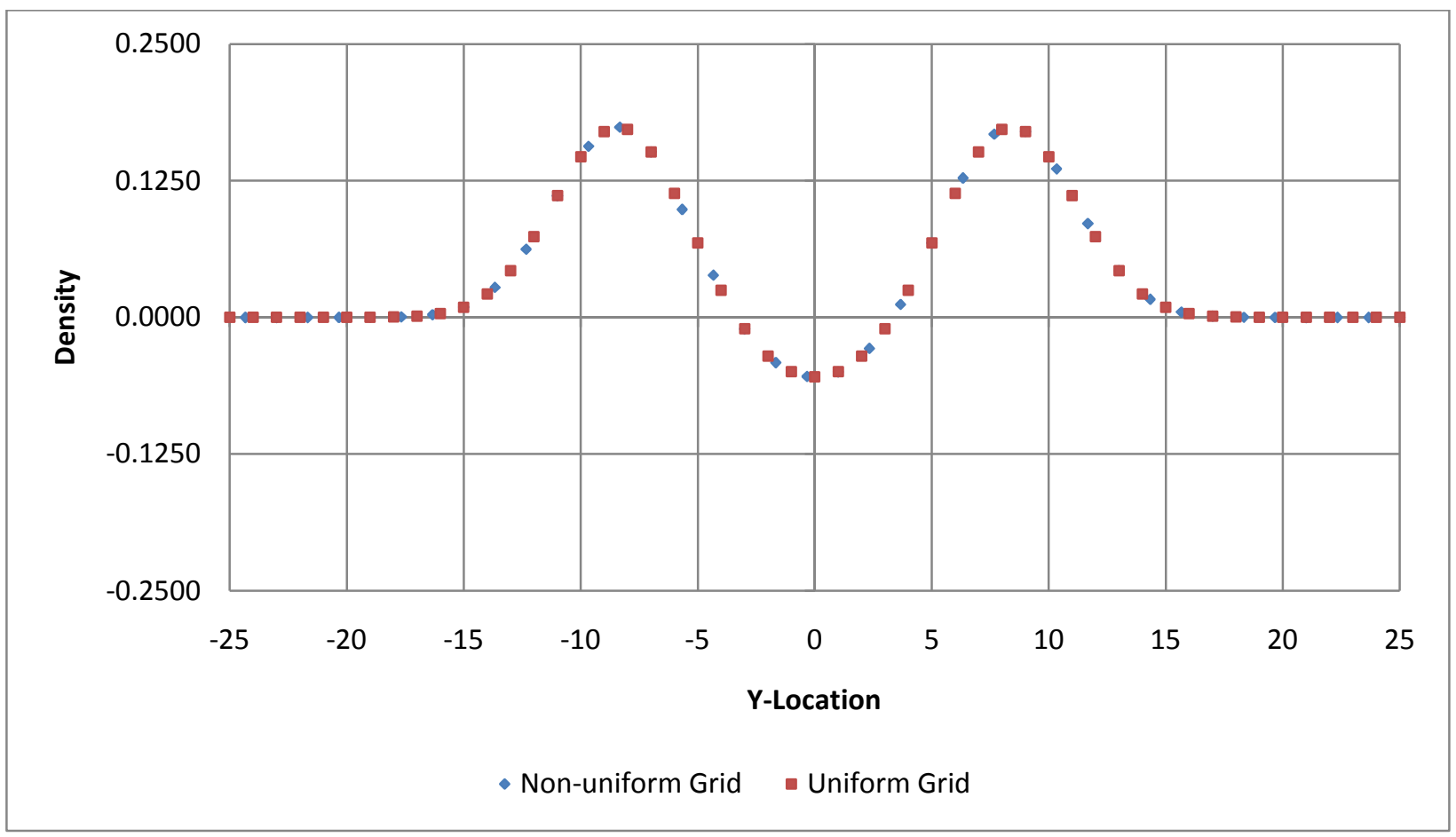

Figure 5.18. Density along line $x=0$ with $M_{x}=0.5$ and $M_{y}=0.0$ at $t=10$.

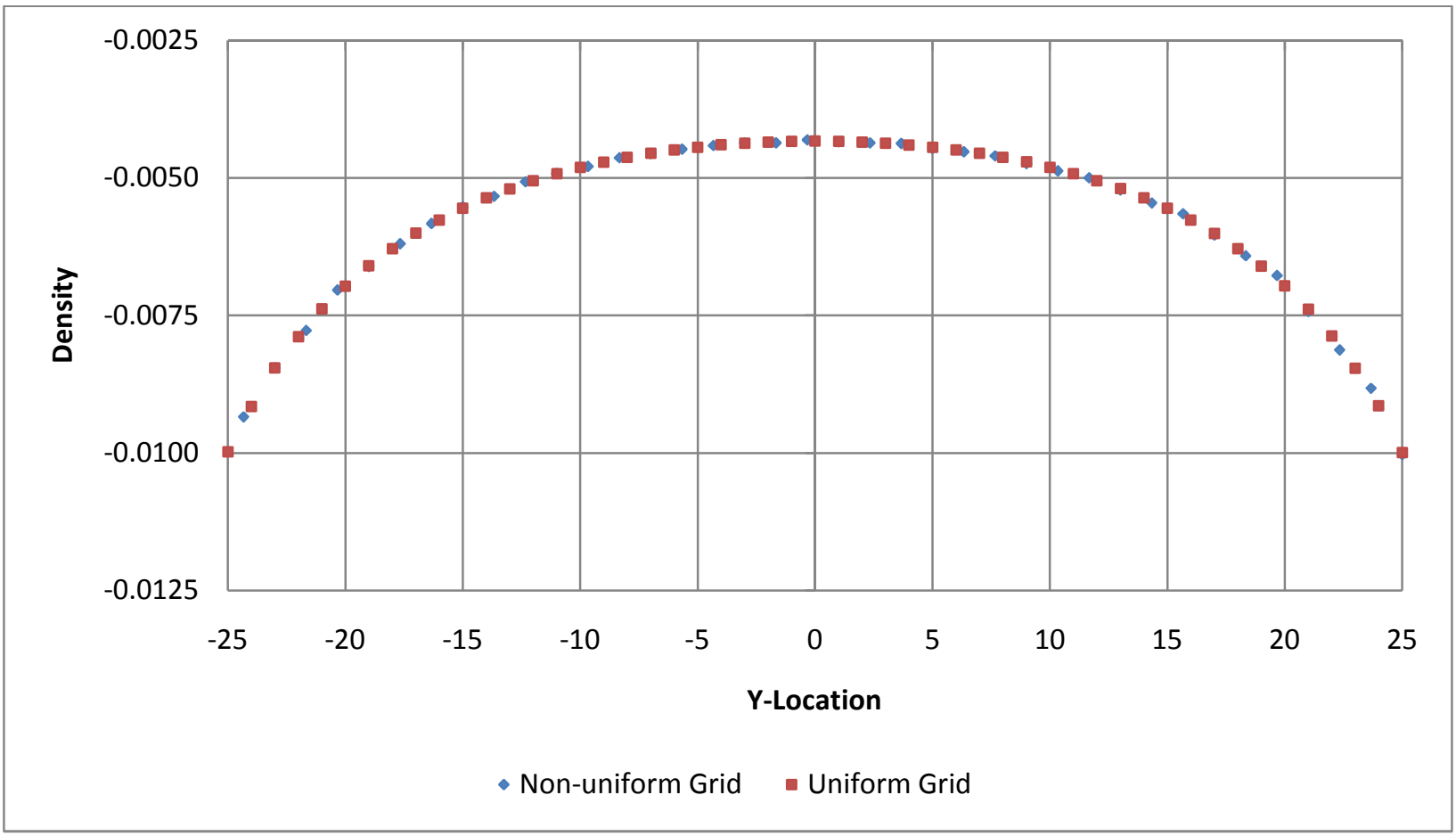

Figure 5.19. Density along line $x=0$ with $M_{x}=0.5$ and $M_{y}=0.0$ at $t=40$. 
(a)

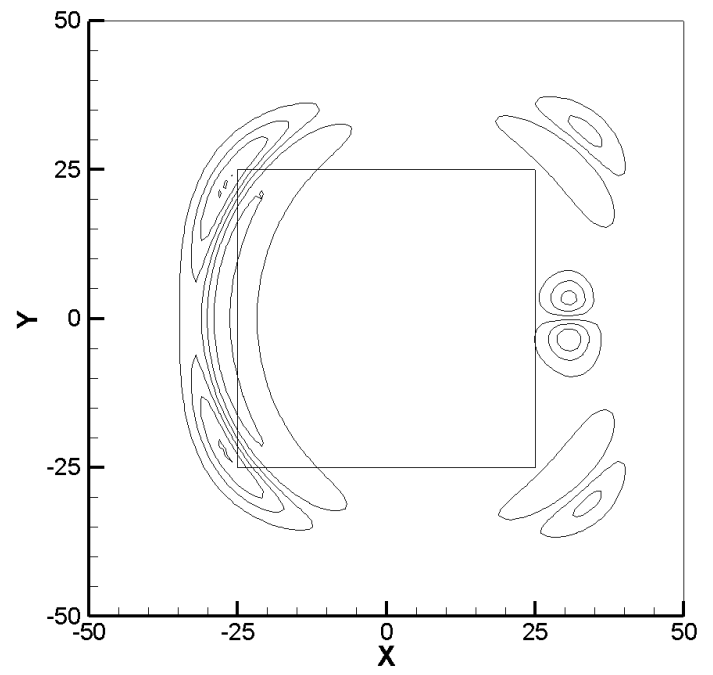

(b)

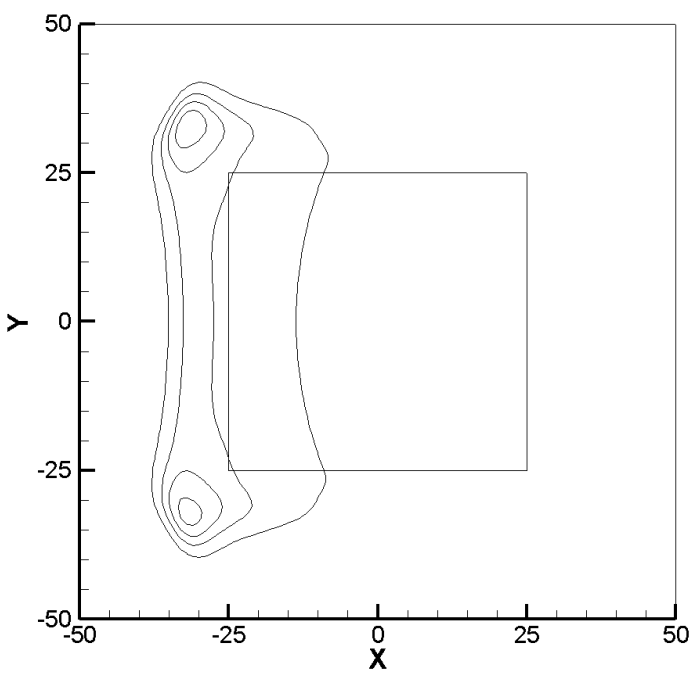

(a)

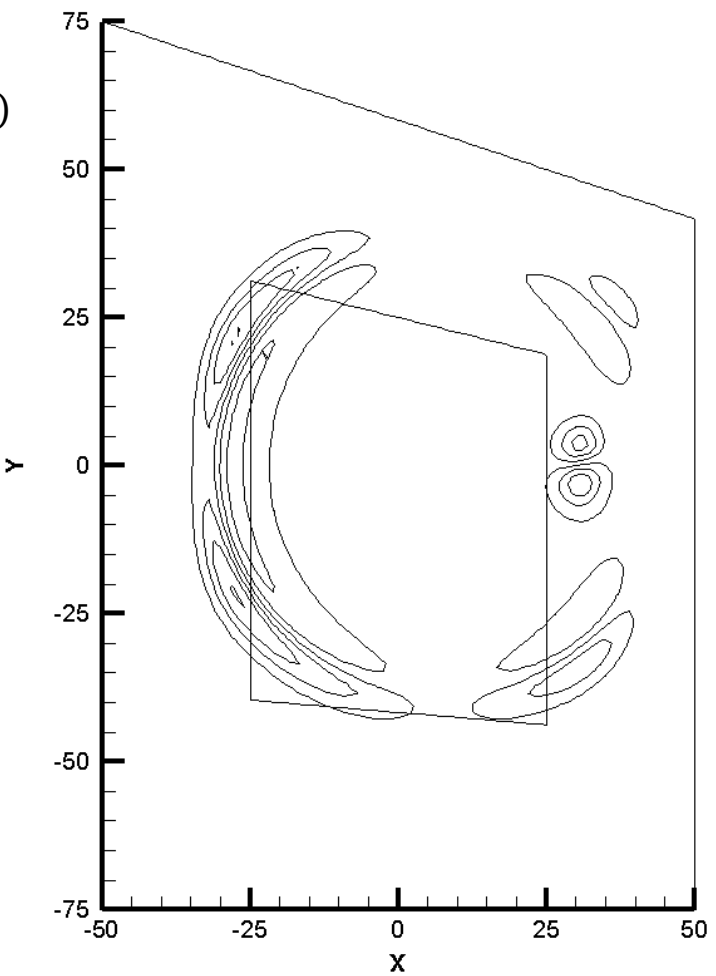

(b)

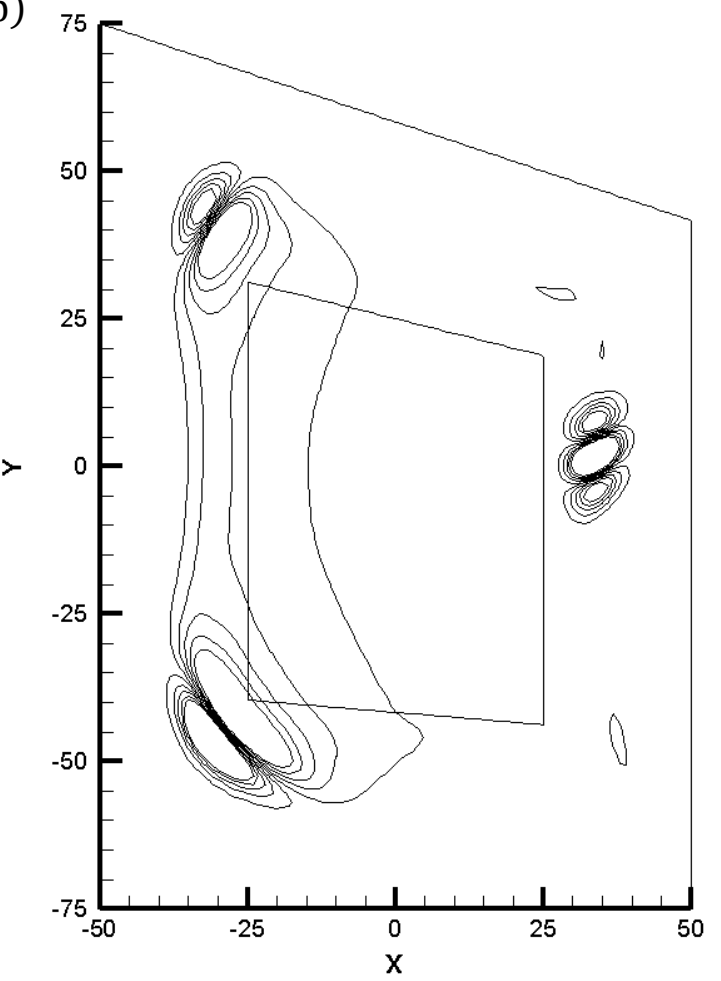

Figure 5.20. Velocity $(u)$ contours at values of \pm 0.1 and \pm 0.05 with uniform and nonuniform grids, showing acoustic and vorticity pulses: $M_{x}=0.5$ and $M_{y}=0.0$ at (a) $t=40$ and $(b) t=70$ 


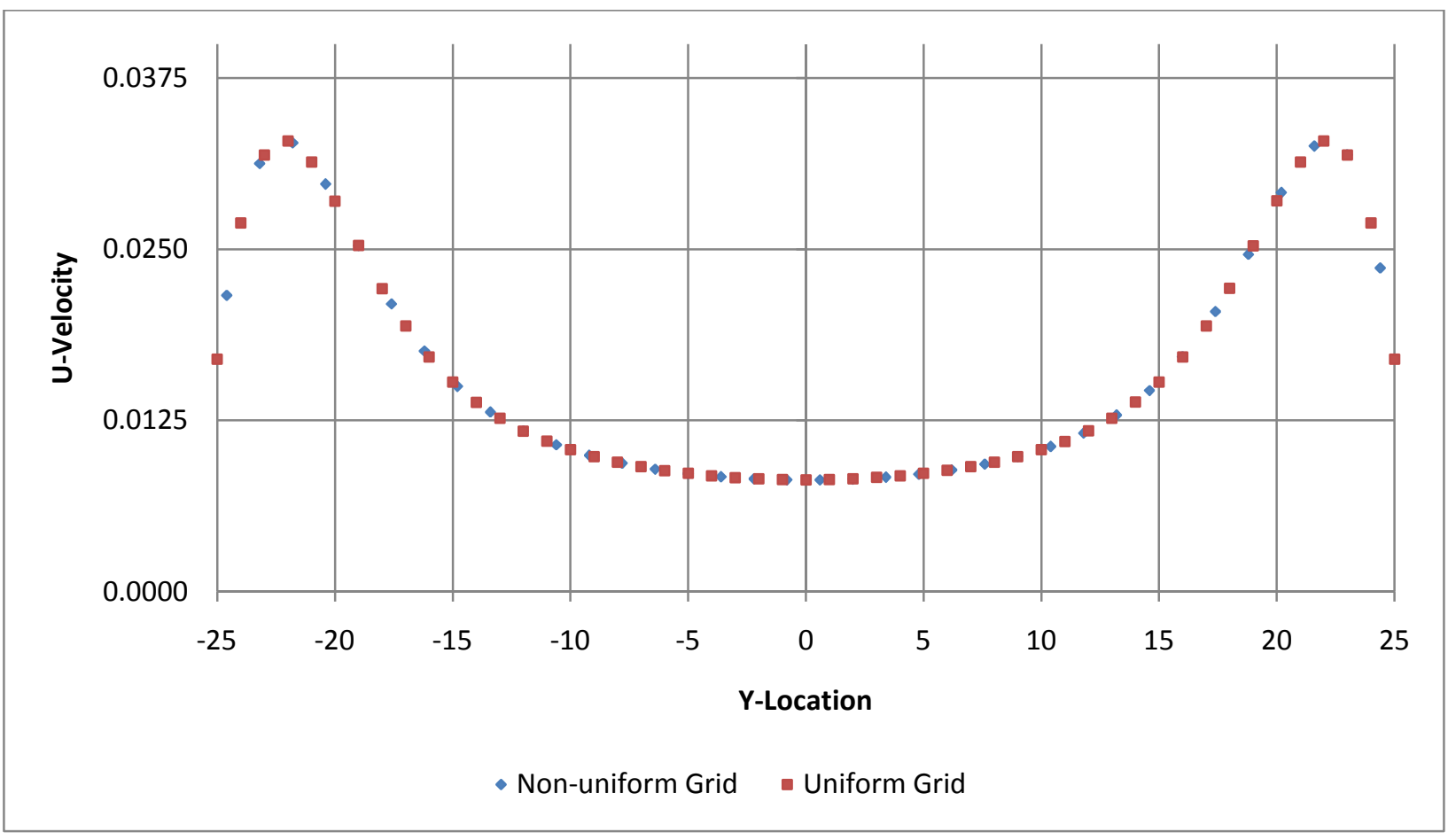

Figure 5.21. Velocity $(u)$ component along line $x=-20$ with $M_{x}=0.5$ and $M_{y}=$ 0.0 at $t=40$

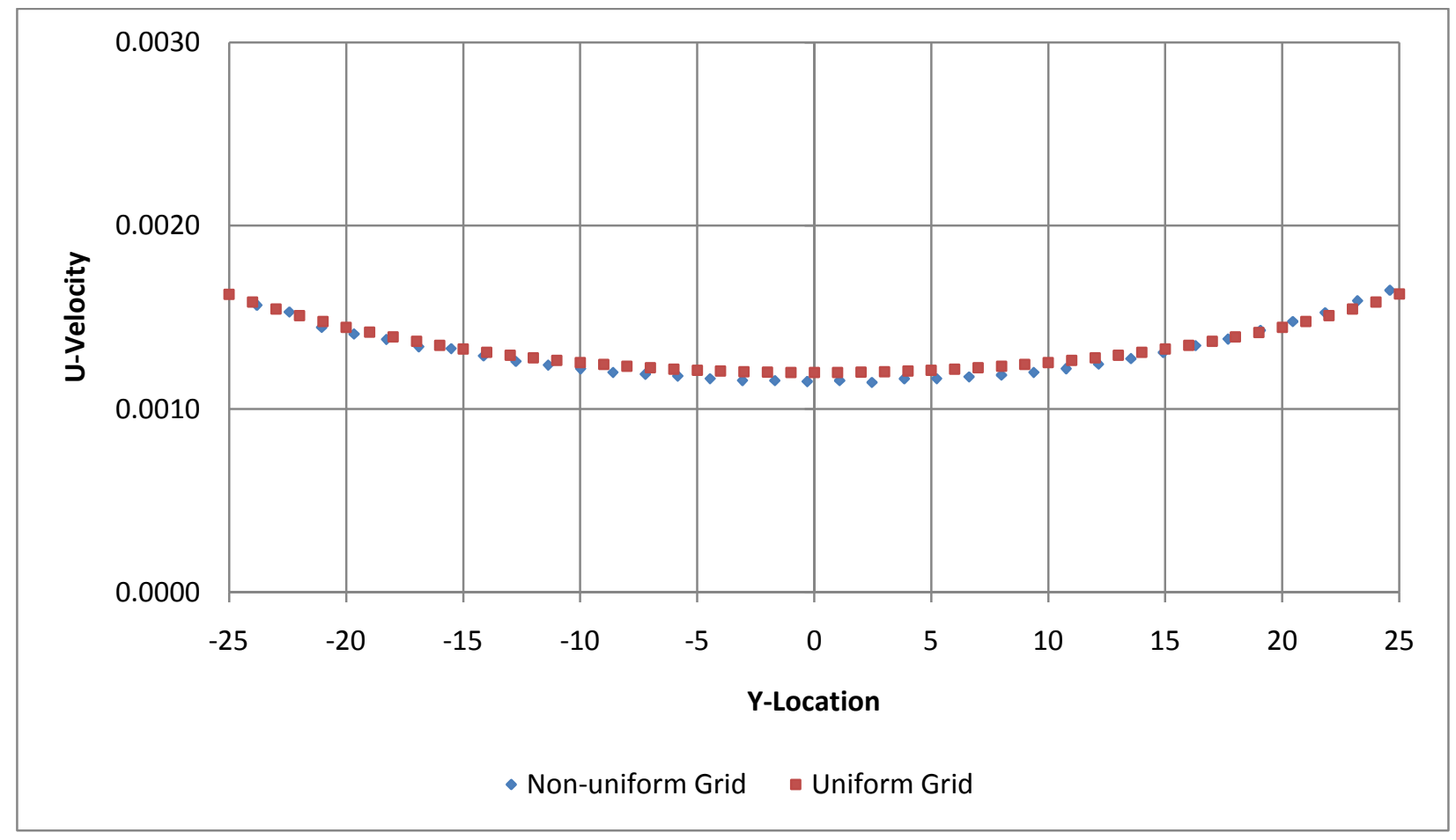

Figure 5.22. Velocity $(u)$ component along line $x=-15$ with $M_{x}=0.5$ and $M_{y}=$ 0.0 at $t=70$. 


\subsubsection{Oblique Mean Flow Case}

Here the PML formulation is validated for a flow in an arbitrary direction. The same initial pulses used in the parallel flow are used, except that the flow has $M_{x}=M_{y}=0.5 \sin (\pi / 4)$ for both the pressure pulse and the acoustic and pressure pulses. The pressure pulse has an acoustic wave located at the origin. For the latter case, the initial center of the acoustic pulse is $\left(x_{a}, y_{a}\right)=(-12.5,12.5)$ and that of the vorticity wave is $\left(x_{b}, y_{b}\right)=(15,15)$. Figure 5.23 shows the density contours for the pressure pulse case. For both grid structures, the waves leave the domain with no reflections. Figures 5.24 and 5.25 show the density along the line $x=0$ at $t=30$ and $t=80$ respectively. From the plots, it can be inferred that the solution obtained from different grid structures are identical for the given identical initial conditions.

The velocity $(u)$ contours are shown in Figure 5.26 . The velocity $(u)$ along the line $x=0$ is shown in Figures 5.27 and 5.28 for $t=30$ and $t=70$ respectively. The solution from the domain with uniform grid matches with that of the non-uniform grid structure.

Figure 5.29 shows the density contours for the acoustic pulse and vorticity pulse case. The density contours along line $x=0$ is shown in Figures 5.30 and 5.31 for $t=10$ and $t=40$ respectively. The solution obtained from the two different grid structures for the given conditions are same.

Figure 5.32 presents the velocity $(u)$ contours where as Figures 5.33 and 5.34 show the velocity $(u)$ along $x=-15$ at $t=40$ and along $x=-20$ at $t=70$ respectively. The contours and the plots show that identical solution is generated despite the 
difference in the grid structures employed in the numerical scheme for a given identical initial conditions. The contours without reflections and the similarity of the solutions demonstrate that a non-rectangular physical domain can be solved in a rectangular computational domain obtained using the equations in the generalized coordinate system. 


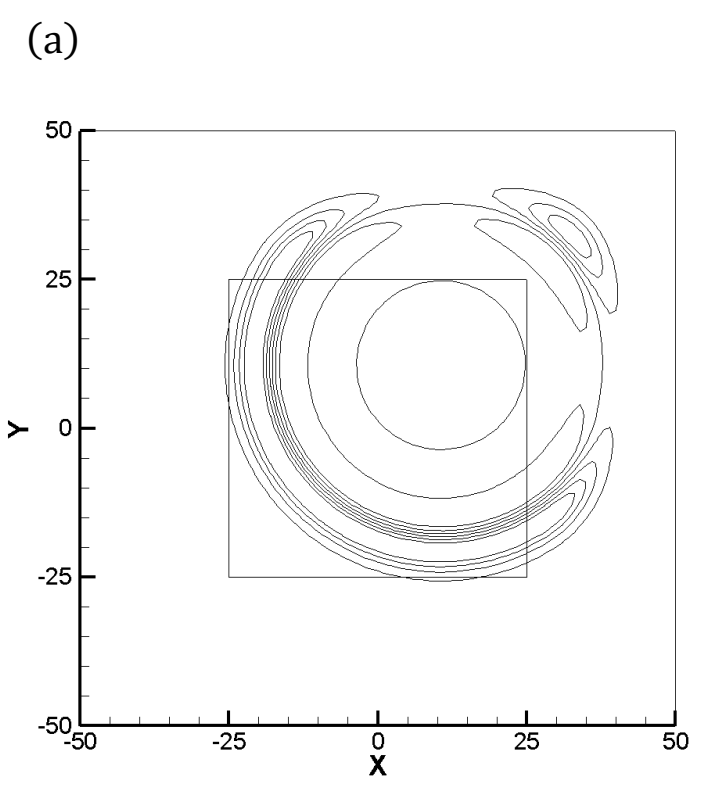

(a)

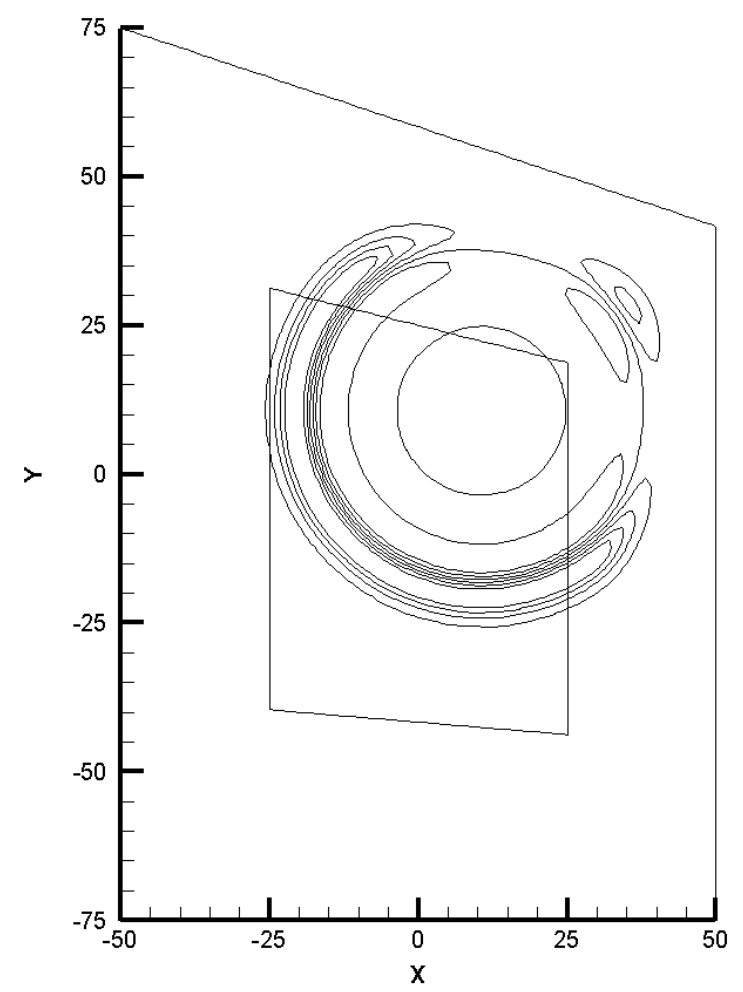

(b)

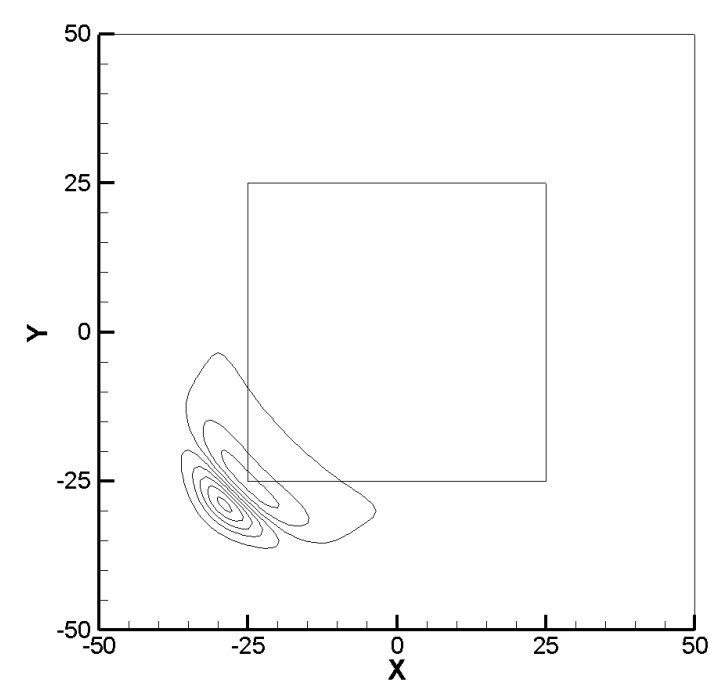

(b)

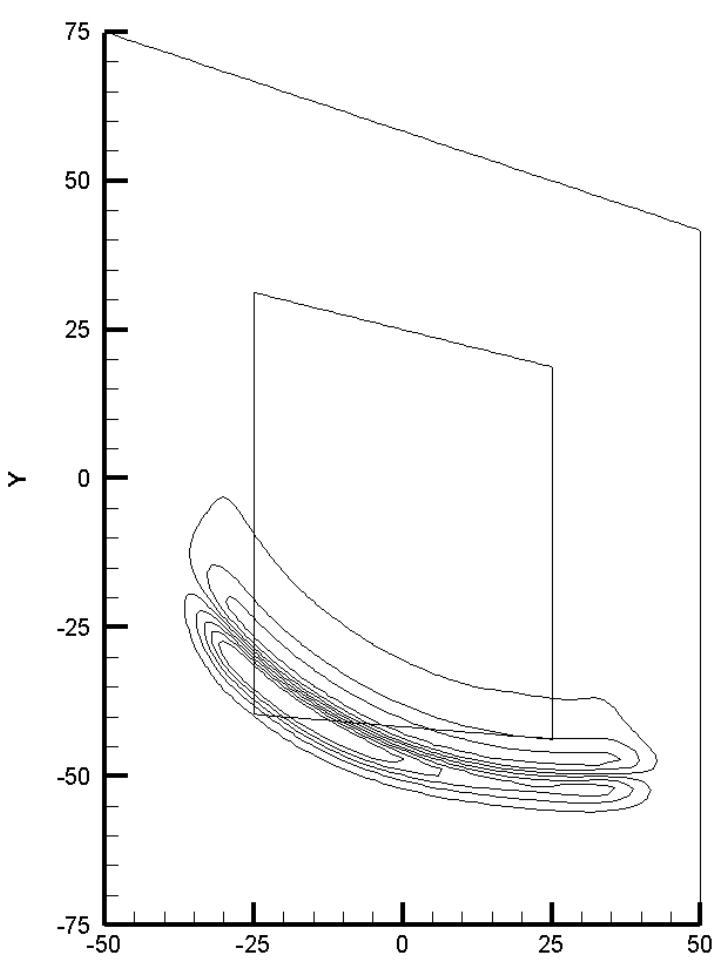

Figure 5.23. Density contours at values of \pm 0.1 and \pm 0.05 with uniform and non-uniform grids, showing acoustic pulse: $M_{x}=0.5 \sin (\pi / 4)$ and $M_{y}=0.5 \sin (\pi / 4)$ at $(a) t=$ 30 and $(b) t=80$. 


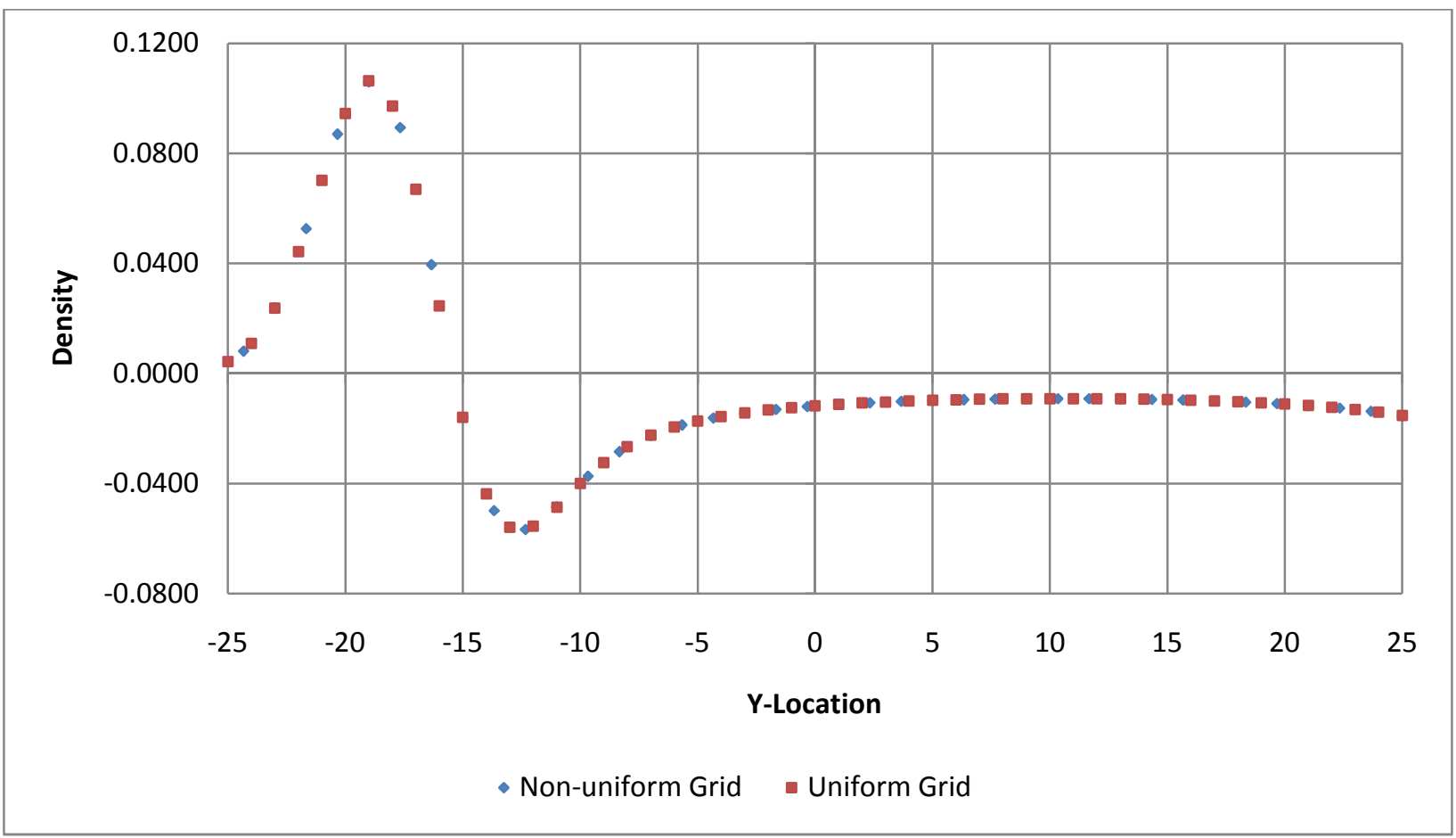

Figure 5.24. Density along line $x=0$ with $M_{x}=0.5$ and $M_{y}=0.5 \sin (\pi / 4)$ at $t=30$.

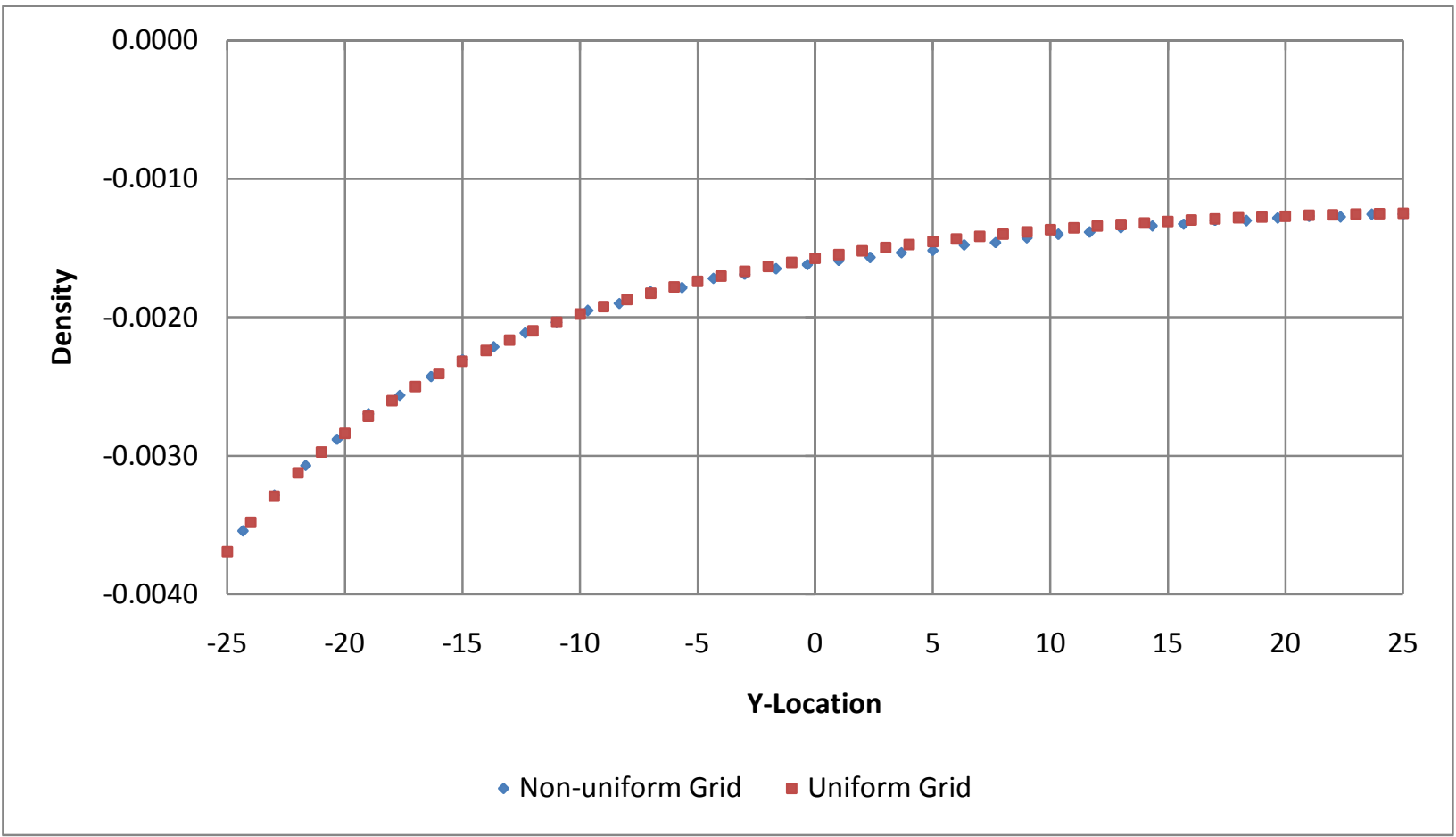

Figure 5.25. Density along line $x=0$ with $M_{x}=0.5 \sin (\pi / 4)$ and $M_{y}=0.5 \sin (\pi /$ 4) at $t=80$. 


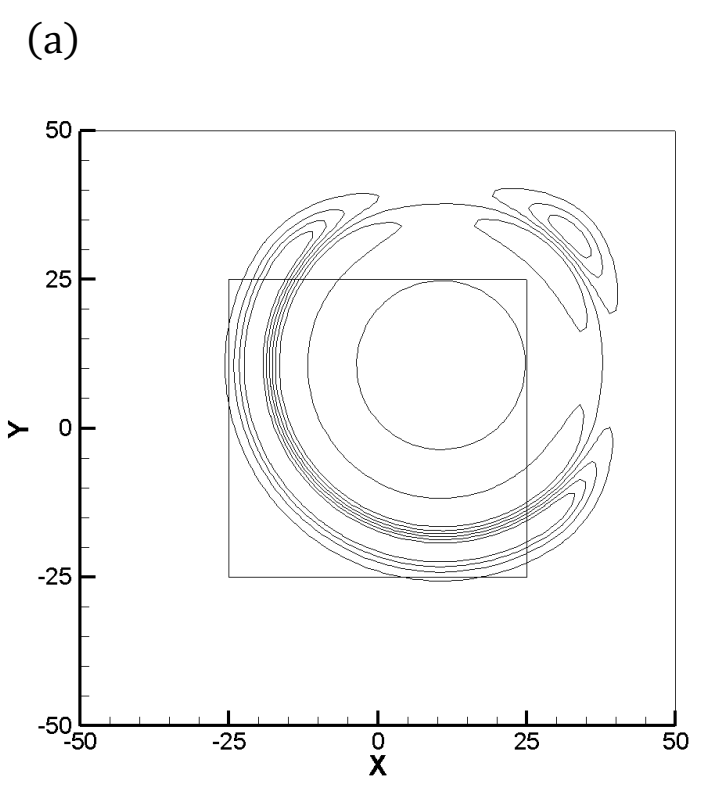

(a)

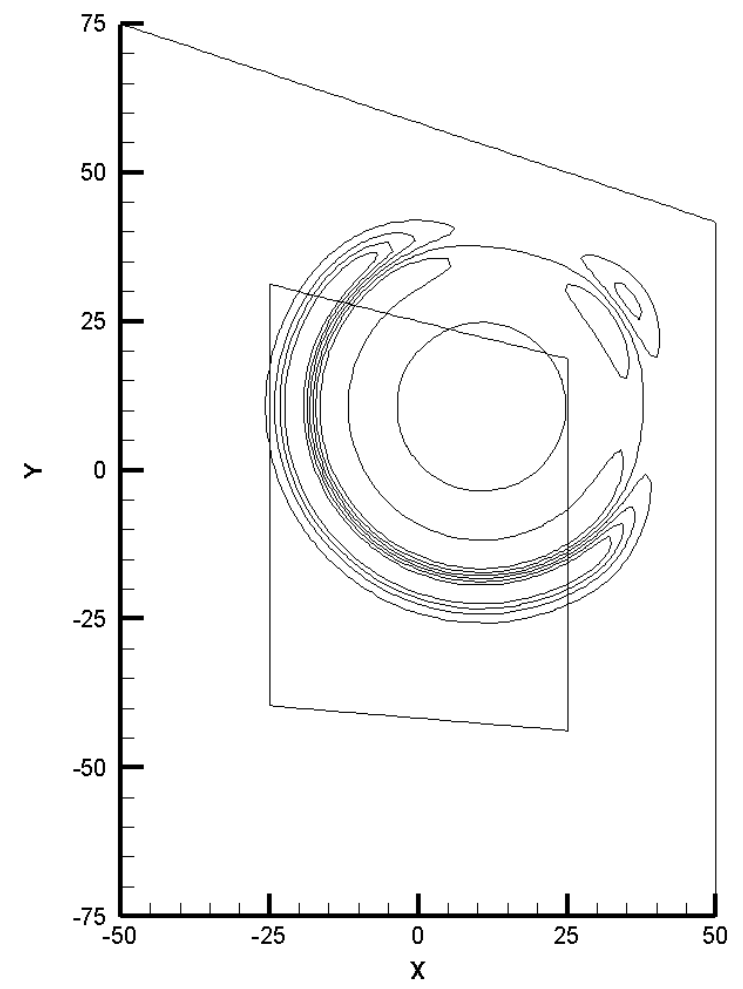

(b)

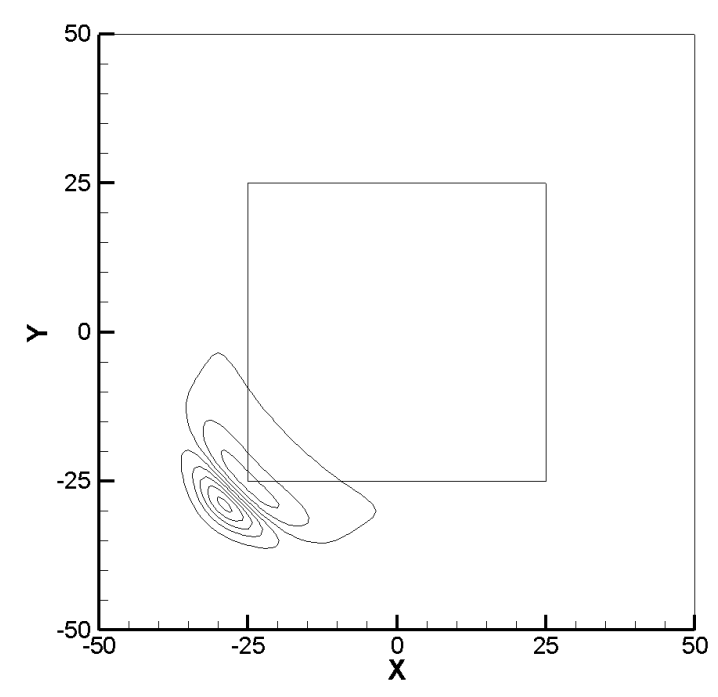

(b)

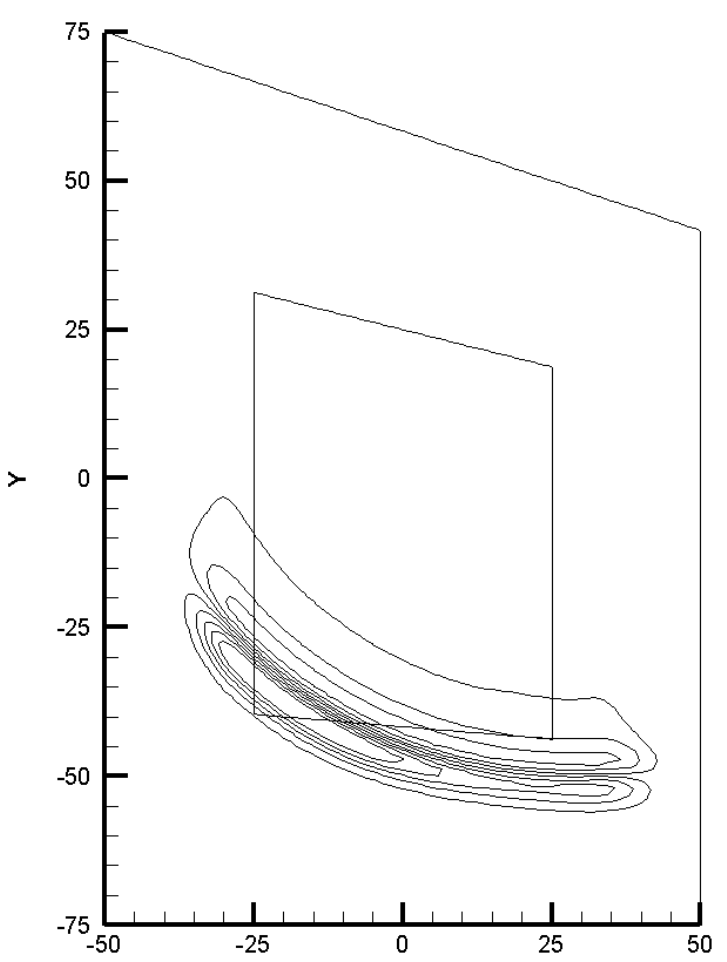

Figure 5.26. Velocity $(u)$ contours at values of \pm 0.1 and \pm 0.05 with uniform and nonuniform grids, showing acoustic pulse: $M_{x}=0.5 \sin (\pi / 4)$ and $M_{y}=0.5 \sin (\pi /$ 4) at (a) $t=30$ and (b) $t=70$. 


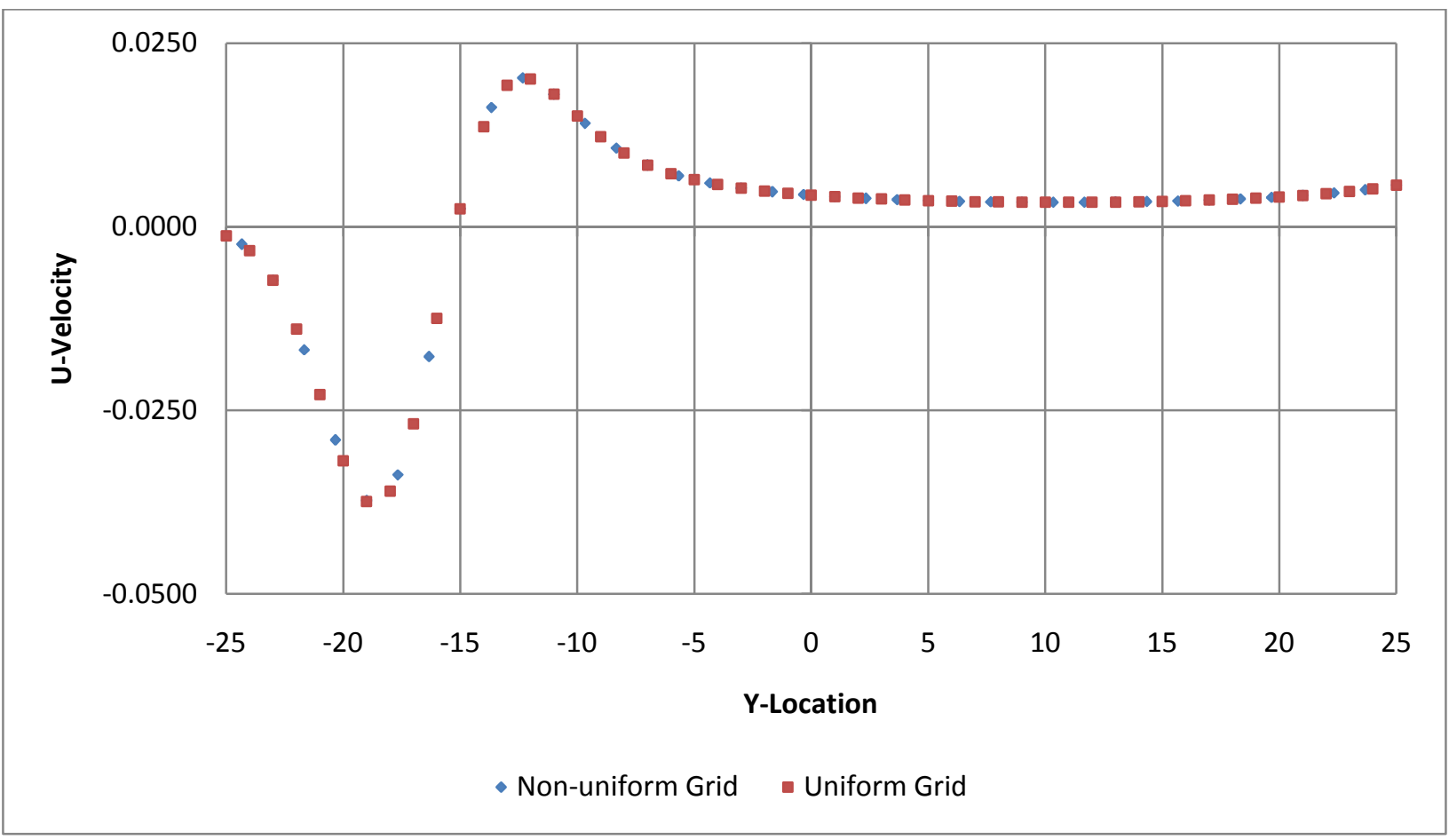

Figure 5.27. Velocity $(u)$ component along line $x=0$ with $M_{x}=0.5 \sin (\pi / 4)$ and $M_{y}=$ $0.5 \sin (\pi / 4)$ at $t=30$.

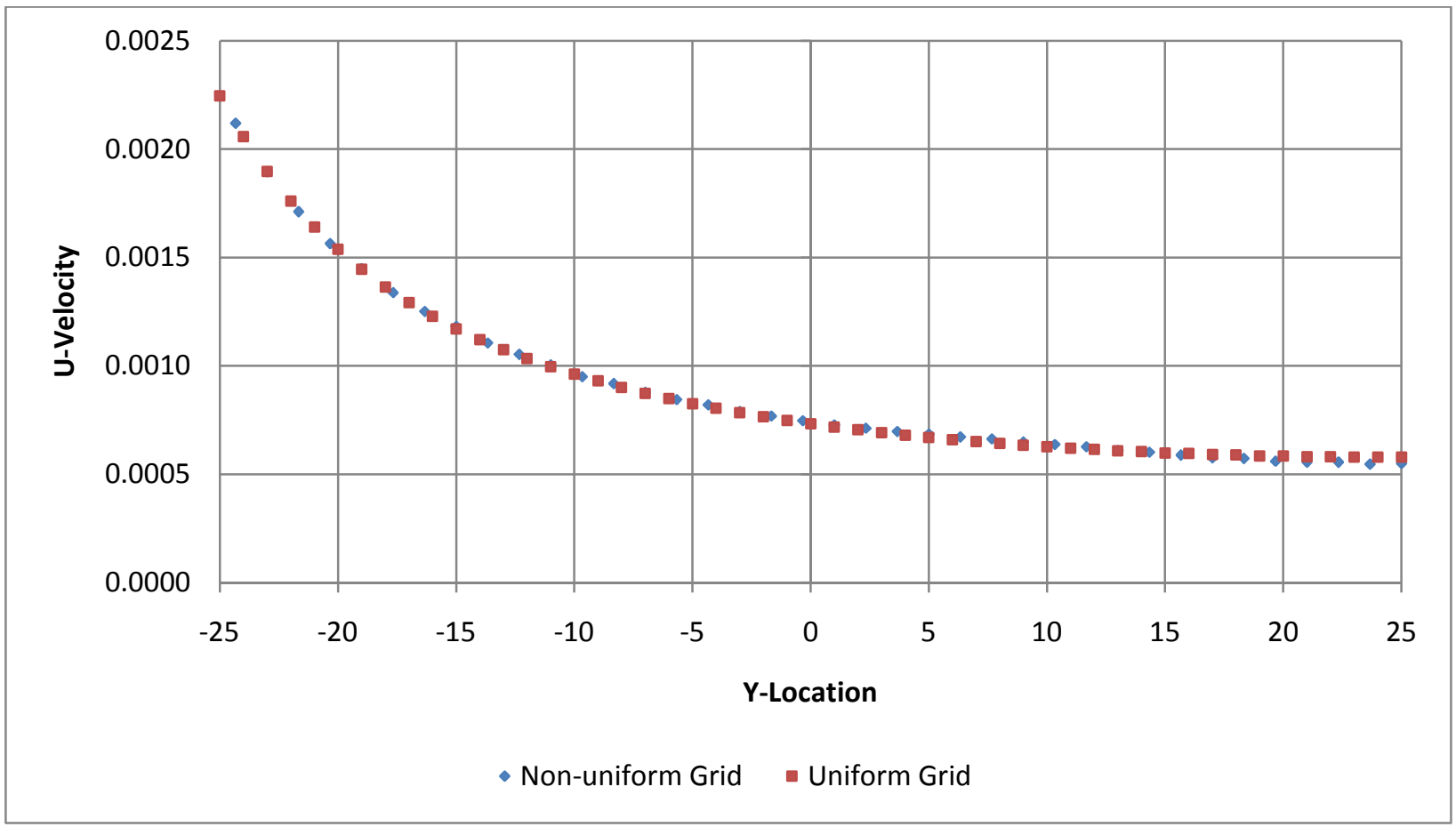

Figure 5.28. Velocity $(u)$ component along line $x=0$ with $M_{x}=0.5 \sin (\pi / 4)$ and $M_{y}=$ $0.5 \sin (\pi / 4)$ at $t=70$. 
(a)

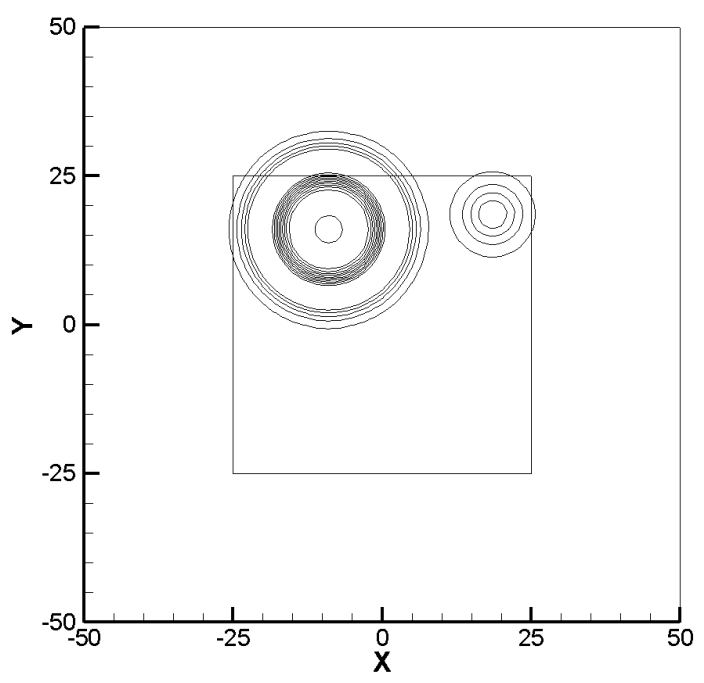

(b)

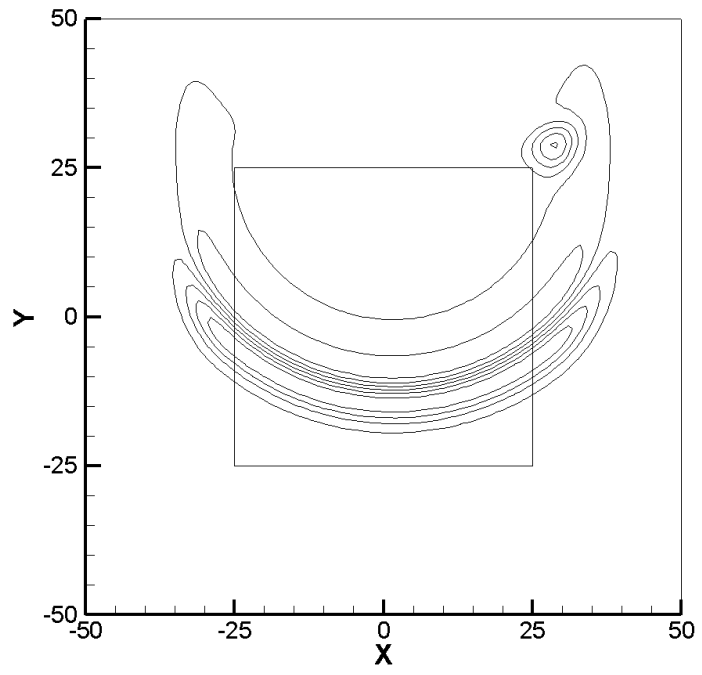

(a)

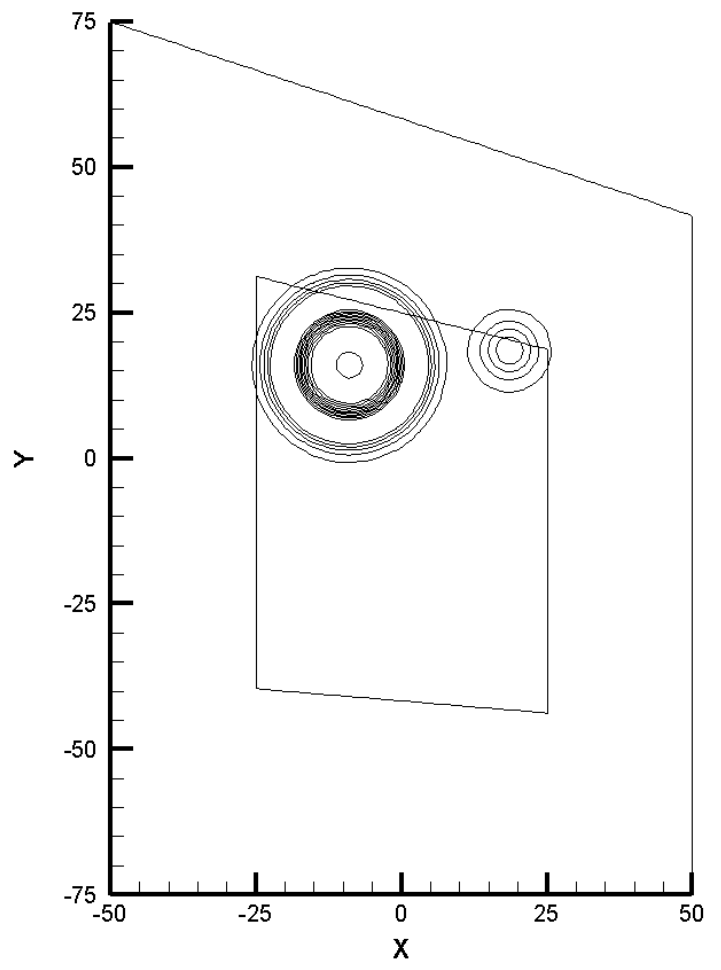

(b)

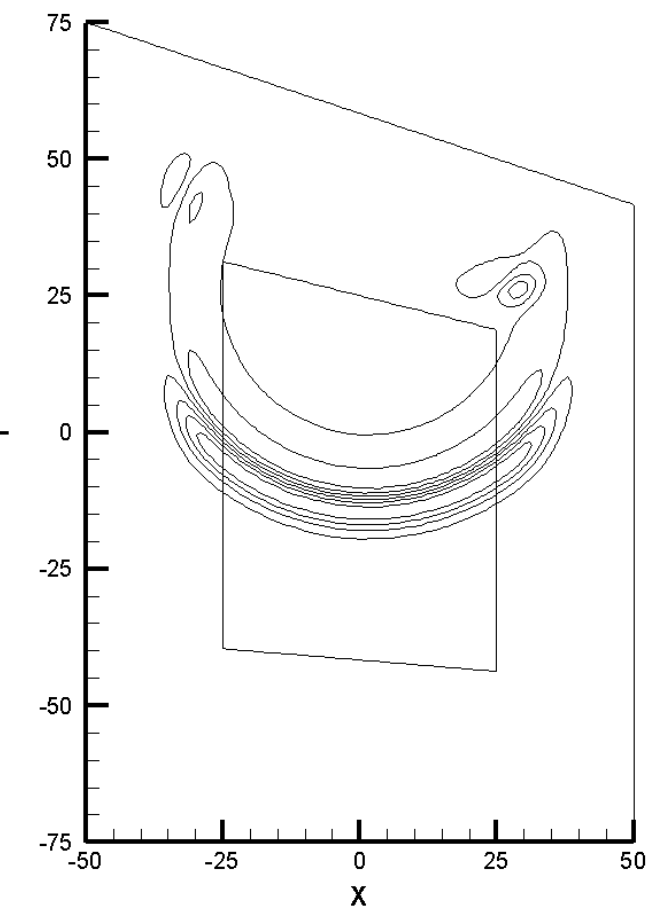

Figure 5.29. Density contours at values of \pm 0.1 and \pm 0.05 with uniform and non-uniform grids, showing acoustic and vorticity pulses: $M_{x}=0.5 \sin (\pi / 4)$ and $M_{y}=0.5 \sin (\pi /$ 4) at (a) $t=10$ and (b) $t=40$. 


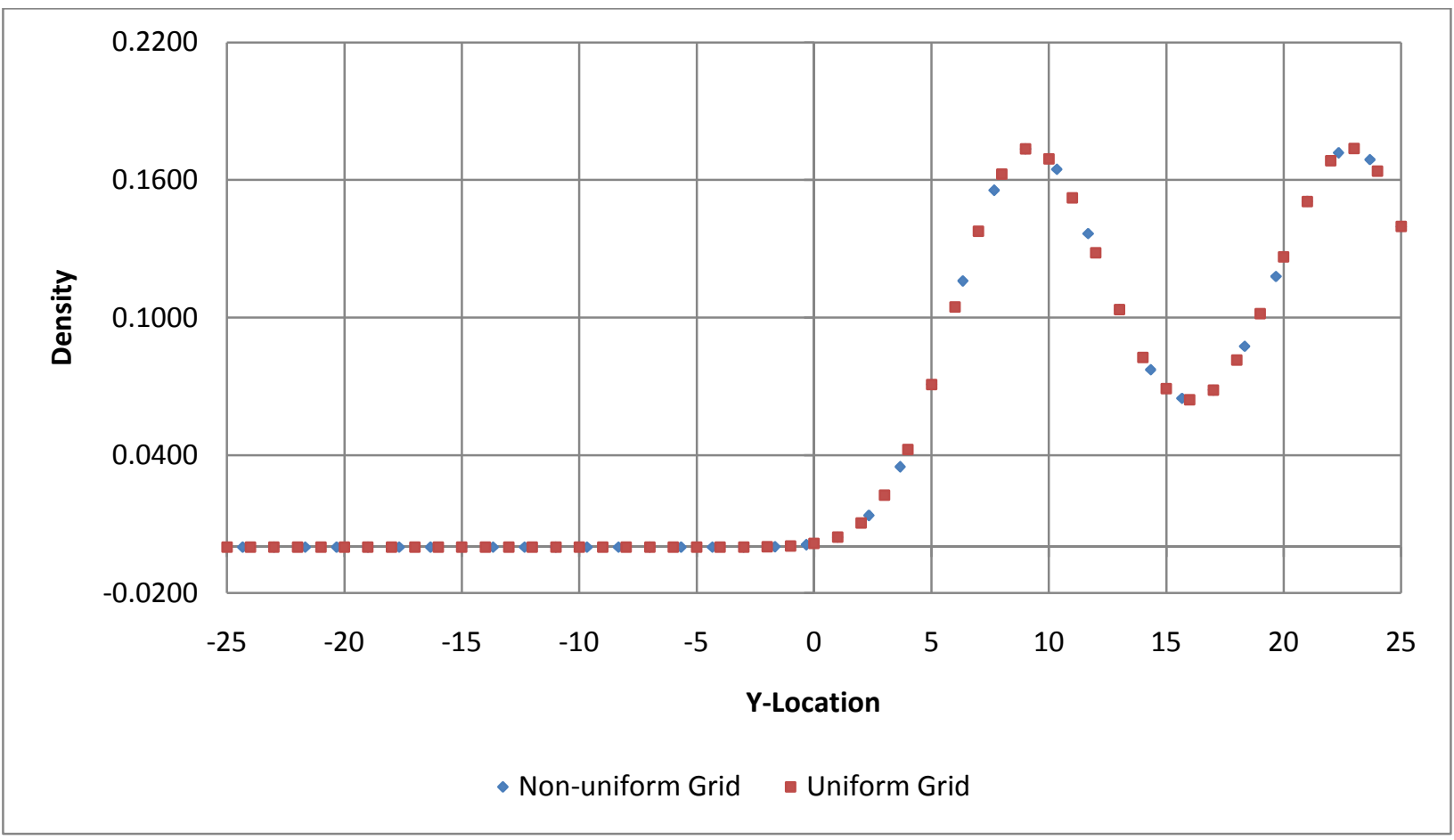

Figure 5.30. Density along line $x=0$ with $M_{x}=0.5 \sin (\pi / 4)$ and $M_{y}=0.5 \sin (\pi /$ 4) at $t=10$.

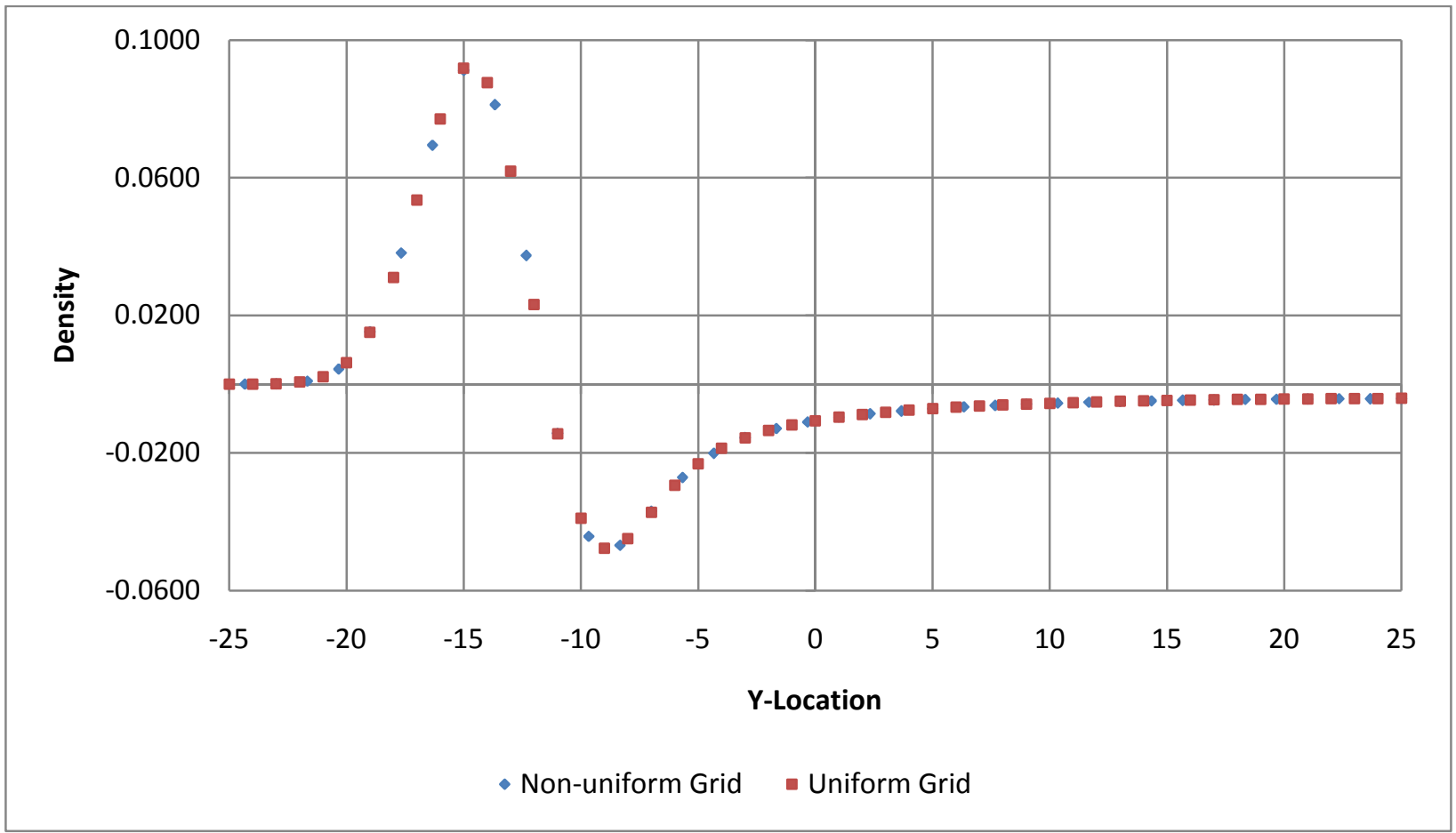

Figure 5.31. Density along line $x=0$ with $M_{x}=0.5 \sin (\pi / 4)$ and $M_{y}=0.5 \sin (\pi /$ 4) at $t=40$. 


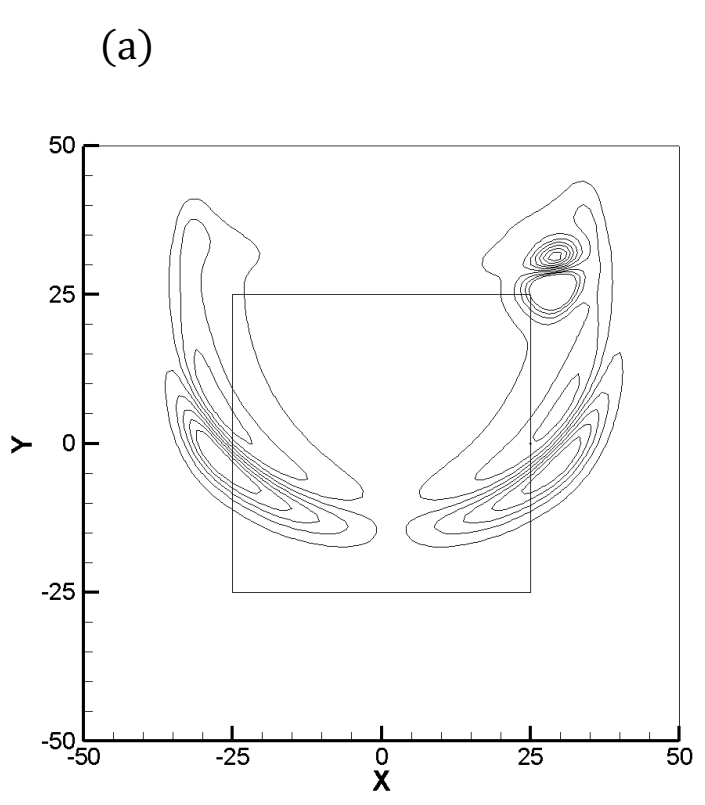

(a)

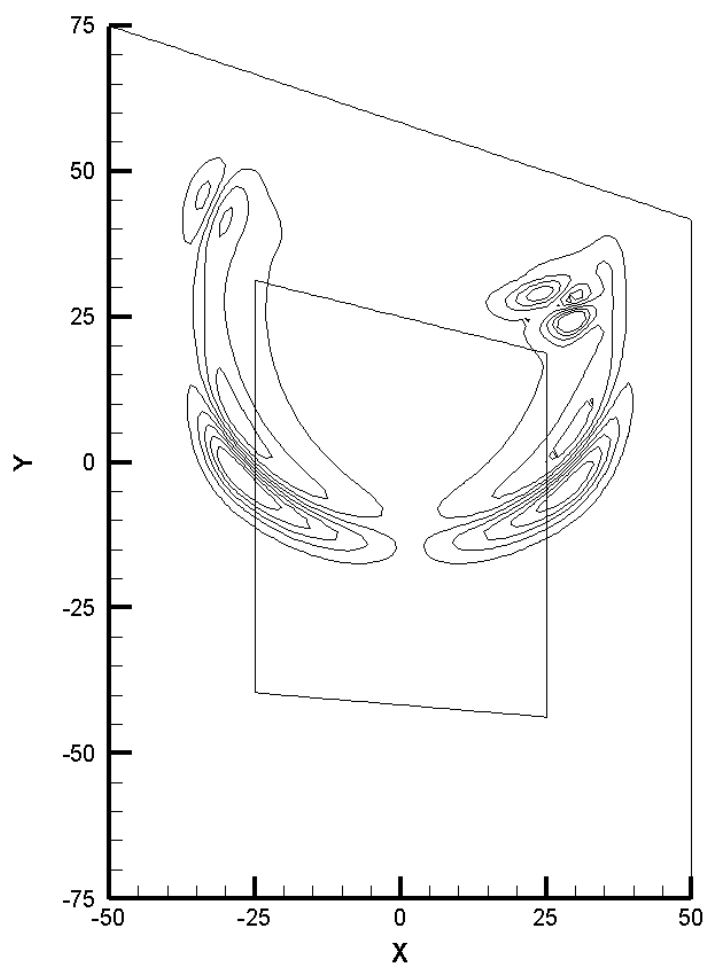

(b)

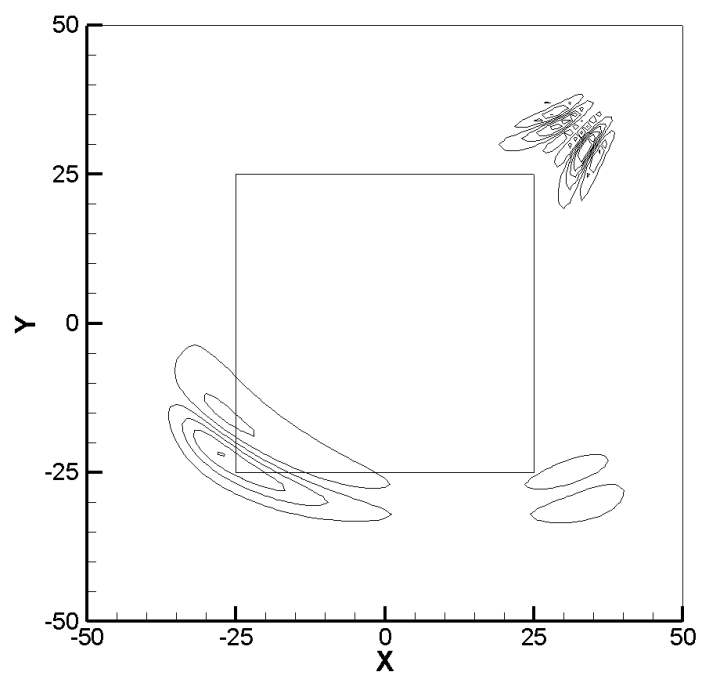

(b)

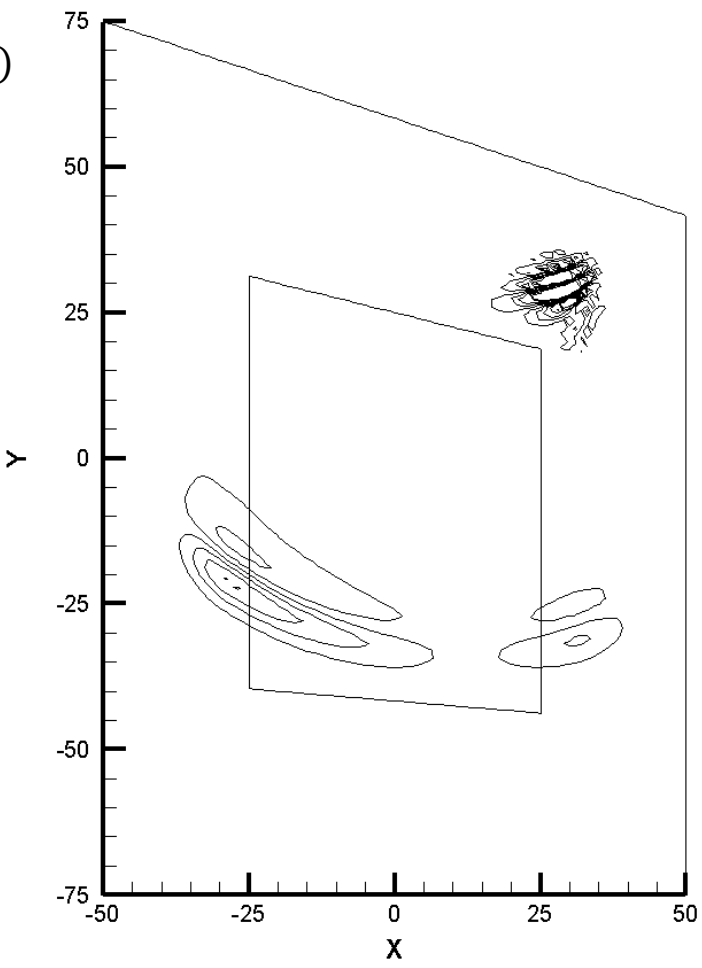

Figure 5.32. Velocity $(u)$ contours at values of \pm 0.05 with uniform and non-uniform grids, showing acoustic and vorticity pulses: $M_{x}=0.5 \sin (\pi / 4)$ and $M_{y}=0.5 \sin (\pi /$ 4) at (a) $t=40$ and (b) $t=70$. 


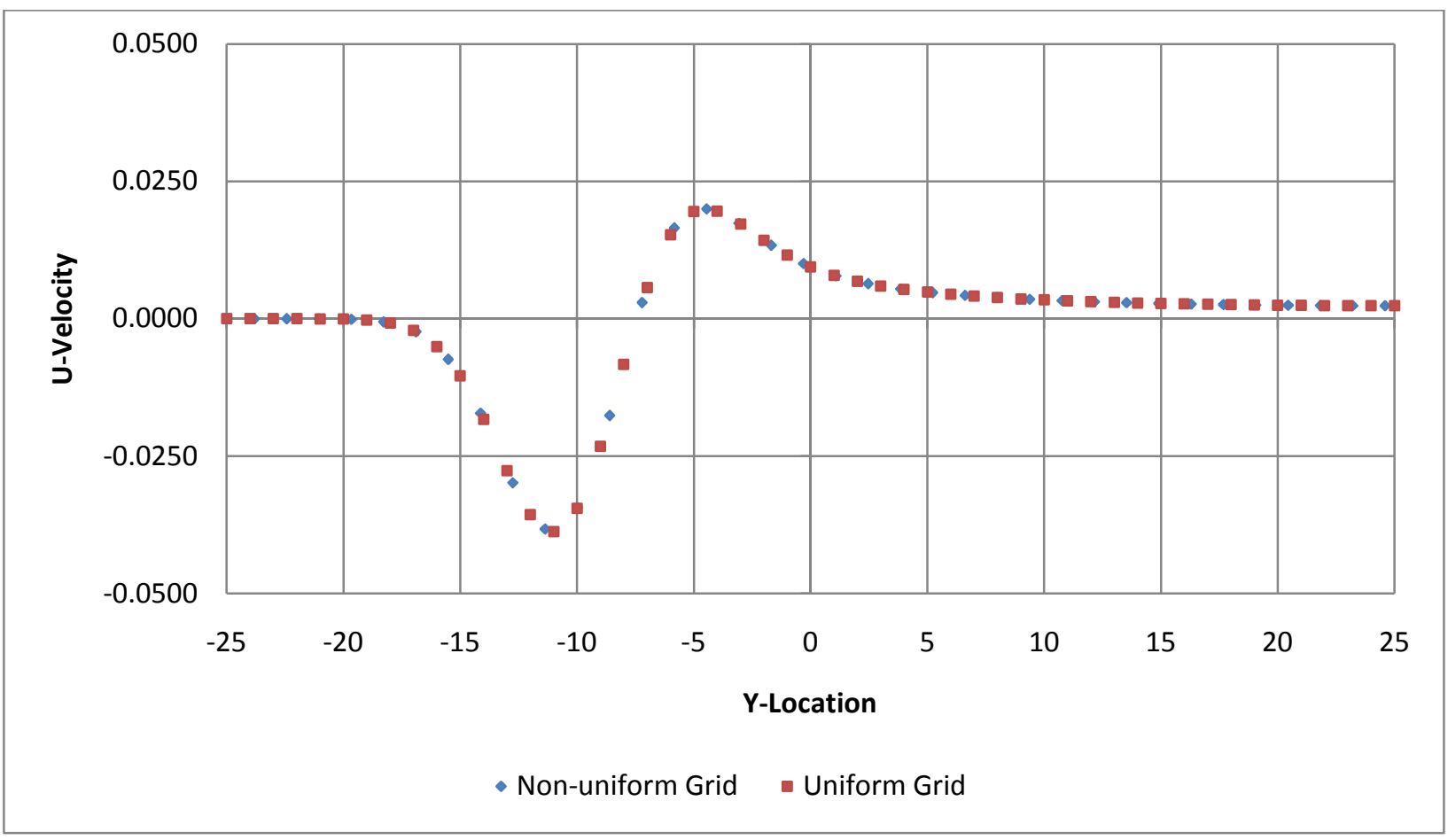

Figure 5.33. Velocity $(u)$ component along line $x=-15$ with $M_{x}=0.5 \sin (\pi /$ 4) and $M_{y}=0.5 \sin (\pi / 4)$ at $t=40$.

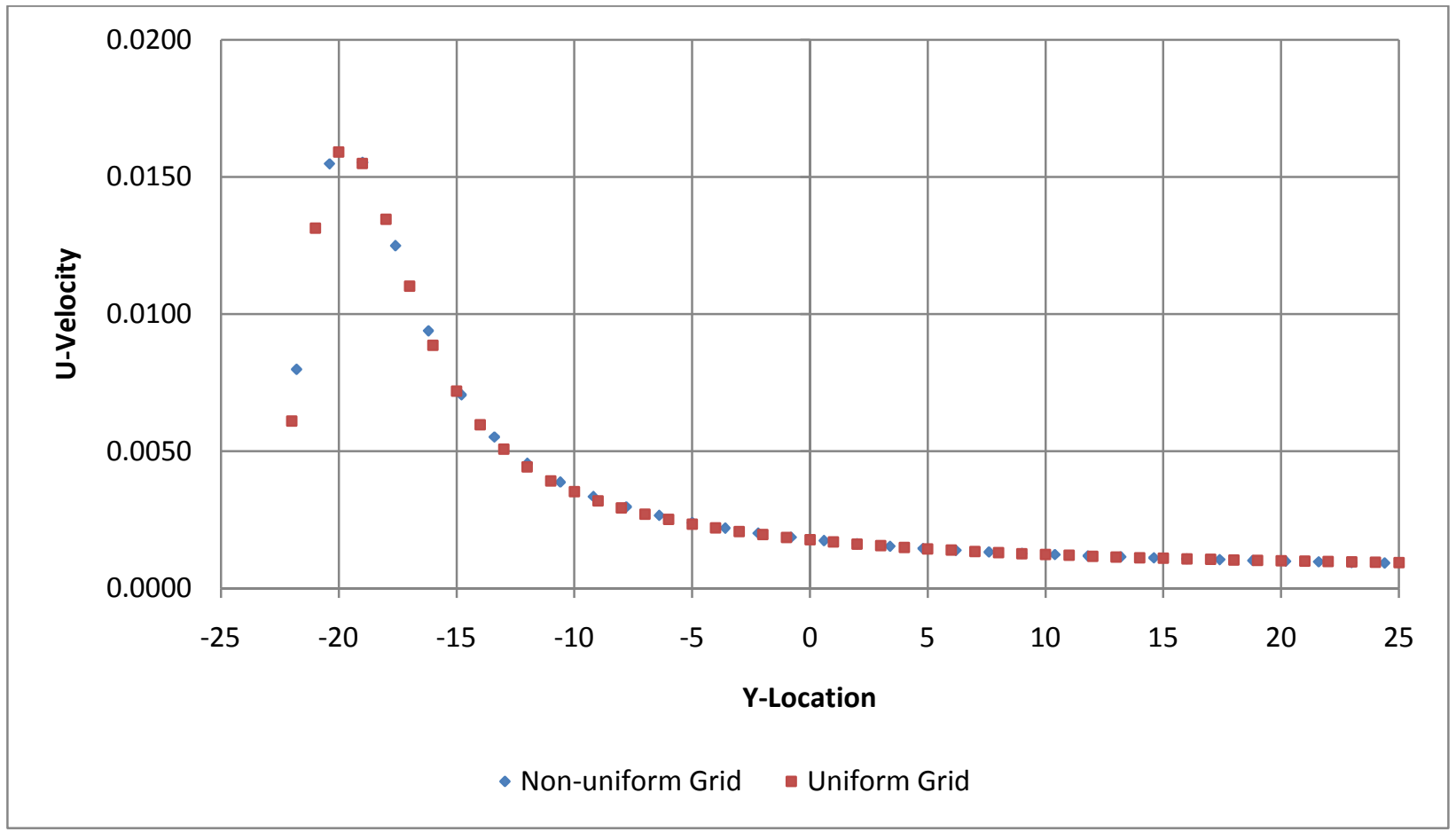

Figure 5.34. Velocity $(u)$ component along line $x=-20$ with $M_{x}=0.5 \sin (\pi /$ 4) and $M_{y}=0.5 \sin (\pi / 4)$ at $t=70$. 


\subsection{Investigation of PML Parameters}

In the previous sections, the PML formulation has been validated both in Cartesian and generalized coordinates. It must be reiterated here that even though the main objective of PML formulation is to develop a reflection-free boundary and accurate solution, the size of the domain also plays a major role in computational fluid dynamics. The larger the domain, the longer will be the computational time, and therefore, the greater will be the cost of analysis. Thus, PML formulation is useful for reducing the domain size and increasing both the efficiency of the analysis and accuracy of the numerical solution. Therefore, from an efficiency point of view, one should know a priori the number of layers to be appended to the interior domain and the amount of damping that needs to be incorporated in the PML domain. The empirical relationship between the damping coefficient and the grid spacing of $\mathrm{Hu}[11]$ mentioned in section 3.2 should be recalled here. However, there is no universal, formulated procedure to estimate these parameters. Therefore, the behavior of the numerical solution with respect to

these parameters, i.e., $\sigma_{\max }, \beta$, and NPML must be investigated. The case from the work of Tam and Auriault [26], as used for the validation of PML in generalized coordinates, was used here for this investigation. However, only the uniform grid for parallel flow, as given in section 5.2.1, was considered. The primitive variable, pressure, was used to analyze the effect of PML parameters on the numerical solution. It must be noted that the values of PML parameters that can help produce accurate solution may vary from one case to another. 


\subsubsection{Effect of PML Layer Thickness}

In order to observe the effect of the number of PML layers, NPML, appended onto the interior domain, the damping coefficients were selected as constant values: $\sigma_{\max }=\beta=2.0$. The NPML used were $5,8,10,15,20$ and 25. Figure 5.35 shows the pressure contours at $t=90$. It can be seen in Figure 5.35(a) that the solution with five PML layers has been contaminated due to reflections. The contamination was reduced as the NPML was increased. The solution with eight PML layers, Figure 5.35(b), is comparatively less spurious than the solution with five PML layers. For NPML of ten upwards, the solution was found to be reflection-free and accurate. It was observed that beyond this value, the NPML had no effect on the solution.

(a) NPML $=5$

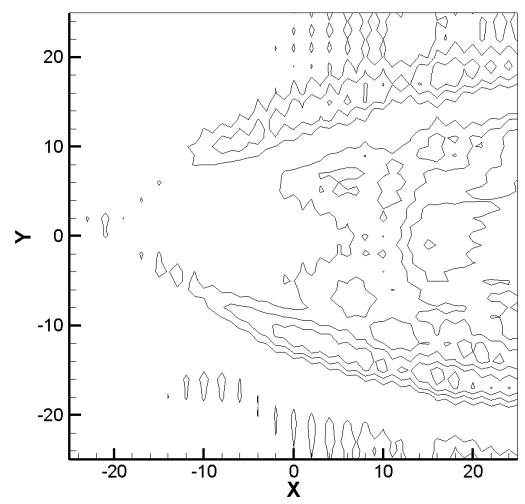

(c) NPML $=10$

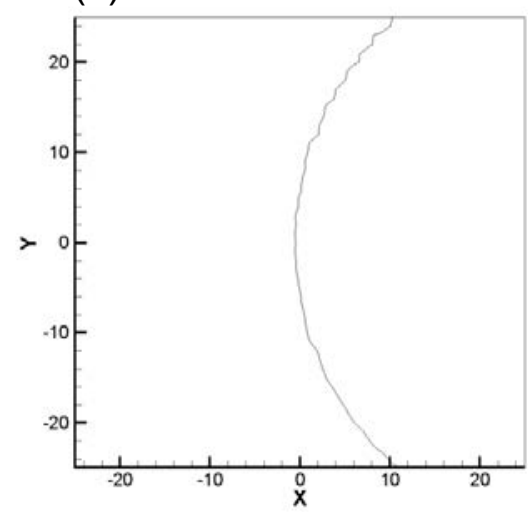

(b) NPML $=8$

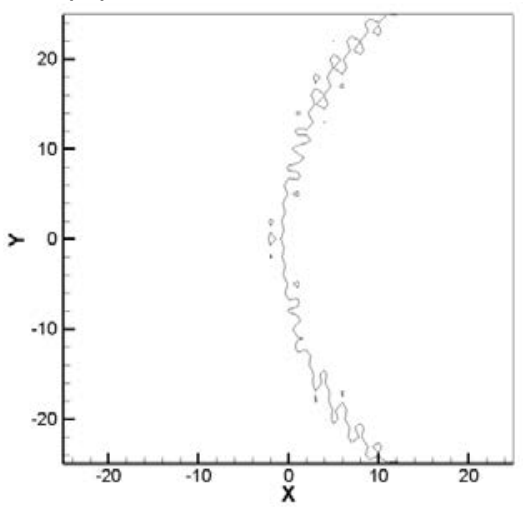

(d) NPML $=15$

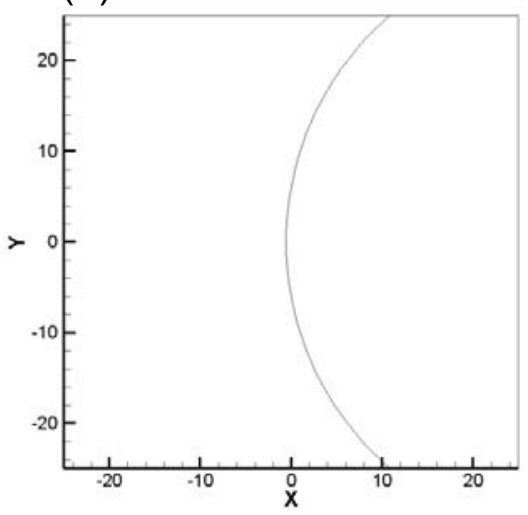


(e) $\mathrm{NPML}=20$

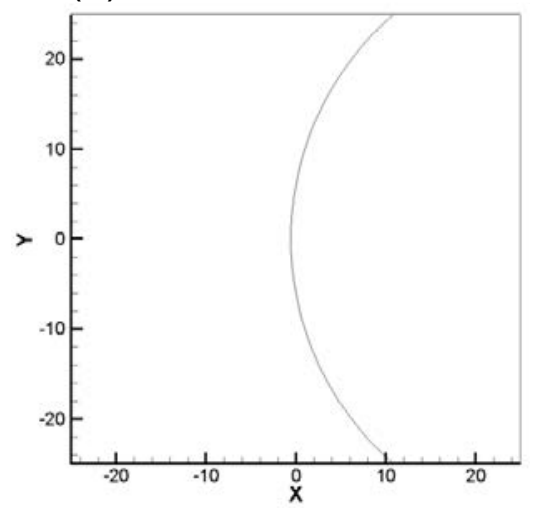

(f) $\mathrm{NPML}=25$

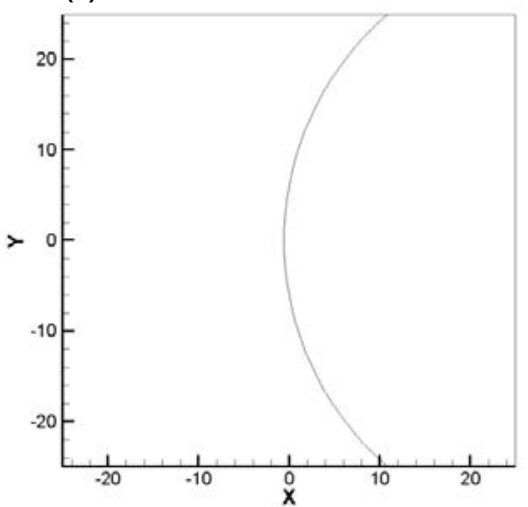

Figure 5.35. Pressure contours at values of \pm 0.001 for various PML thicknesses, $\sigma_{\max }=2.0$, and $\beta=2.0: M_{x}=0.5$ and $M_{y}=0.0$ at $t=90$.

Figure 5.36(a) and (b) shows the pressure along line $y=0$ for various PML thicknesses at $t=50$ and $t=100$, respectively. The oscillations in the solution are quite obvious from the plots and are due to the waves reflected at the boundaries. The contamination was reduced as the NPML was increased. The solution with eight PML layers, Figure 5.36, is comparatively less spurious than the solution with five PML layers. From the plots, it can be further ascertained that, interestingly, after a certain level, no changes in the solution is seen with the increase of NPML. It was observed that solutions with NPML of ten upwards compare well with the reference solution.

Considering NPML of ten for an accurate solution, in this case, a $20 \%$ increase in grid points in each direction provides a reflection-free solution, which does not increase the computational time by a large amount. And this additional computational time can be compromised with the accuracy of the solution obtained with PML. 

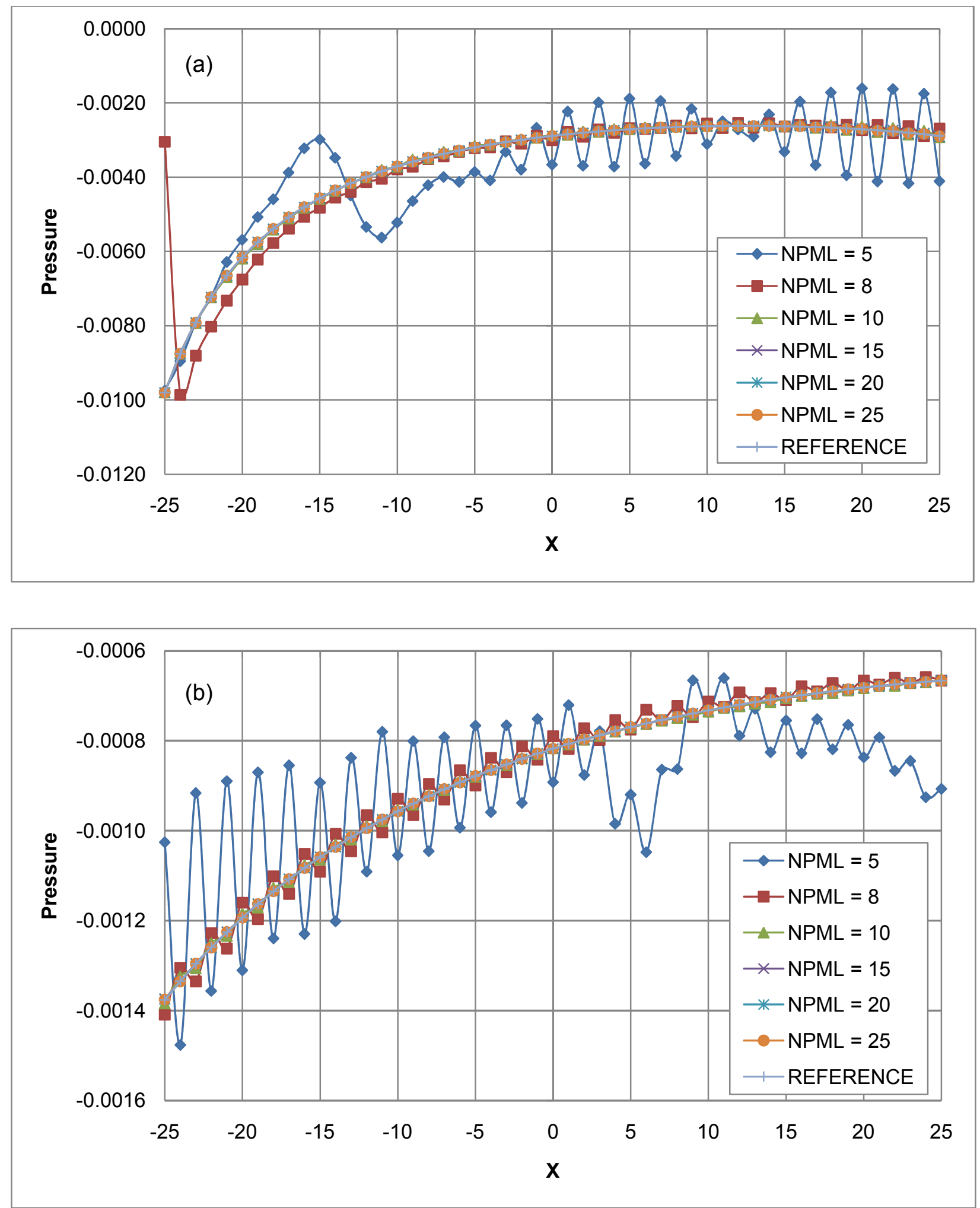

Figure 5.36. Pressure along $y=0$ line with PMLs of various thicknesses, $\sigma_{\max }=2.0$, and $\beta=2.0: M_{x}=0.5$ and $M_{y}=0.0$ at $(a) t=50$ and $(b) t=100$. 


\subsubsection{Effect of Amount of Damping}

To investigate the effect of damping, $\sigma_{\max }$, the amount of damping in the PML, the NPML and $\beta$ were fixed at NPML $=25$ and $\beta=2.0$. The pressure contours showing the solution at $t=100$ is presented in Figure 5.37 for $\sigma_{\max }$ of $0.1,0.5,1.0,1.5,2.0$, and 2.5. The solution behaves in the same way as it did for NPML. The reflections are large, and the solution is highly inaccurate for low values of $\sigma_{\max }$, i.e., for small amount of damping, and the accuracy of the solution increases with an increase in the amount of damping imposed on the PML domain. From $\sigma_{\max }=2.0$ upwards, reflection-free and accurate solutions were attained. For this case, increasing $\sigma_{\max }$ beyond a value of 2 had no effect. It should be noted that changing $\sigma_{\max }$ did not affect the efficiency of the code in terms of computational time. Figures $5.38(a)$ and (b) shows the pressure along line $y=0$ for various values of $\sigma_{\max }$ at $t=50$ and $t=100$, respectively. Although the solutions of different time levels were considered, it can be easily inferred that the observations made from the contour plots were ascertained by the solution plots presented in Figures 5.37(a) and (b). 
(a) $\sigma_{\max }=0.1$

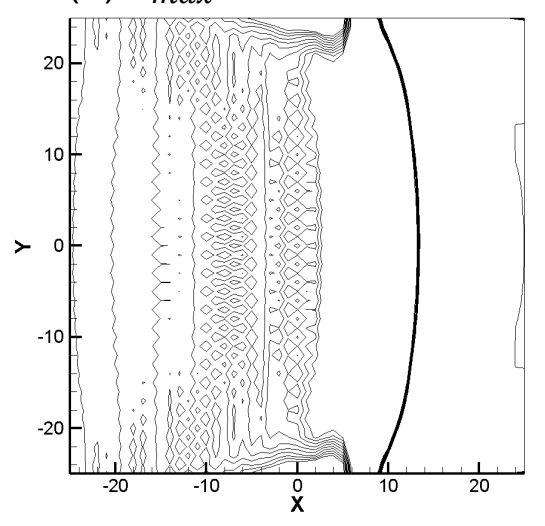

(c) $\sigma_{\max }=1.0$

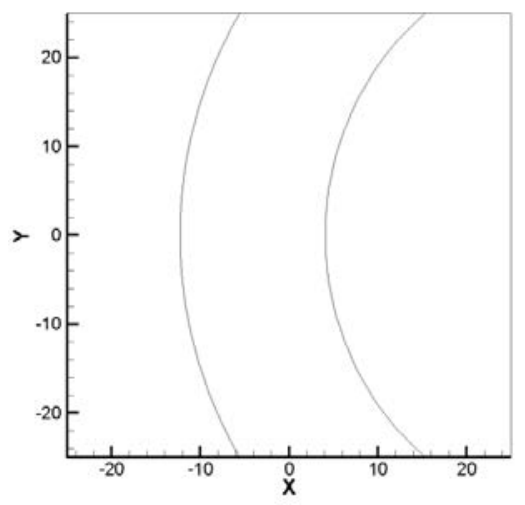

(e) $\sigma_{\max }=2.0$

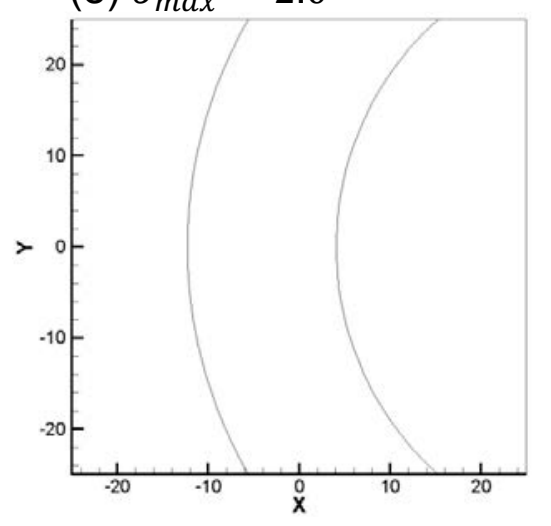

(b) $\sigma_{\max }=0.5$

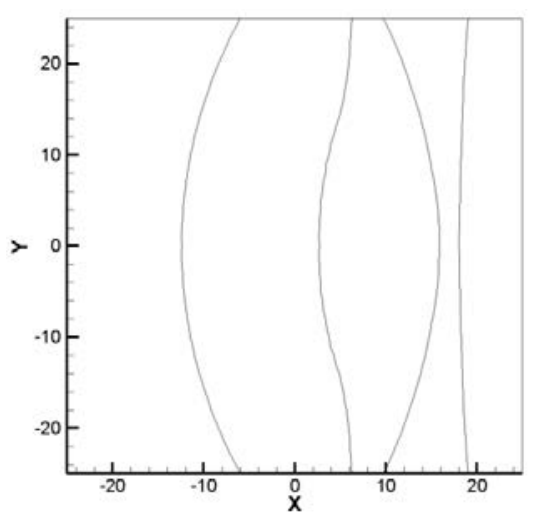

(d) $\sigma_{\max }=1.5$

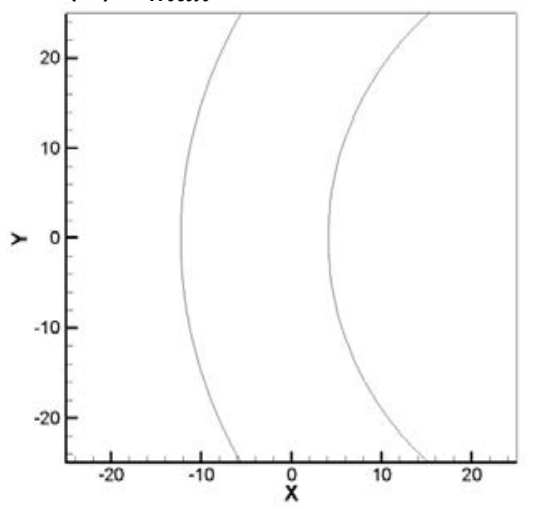

(f) $\sigma_{\max }=2.5$

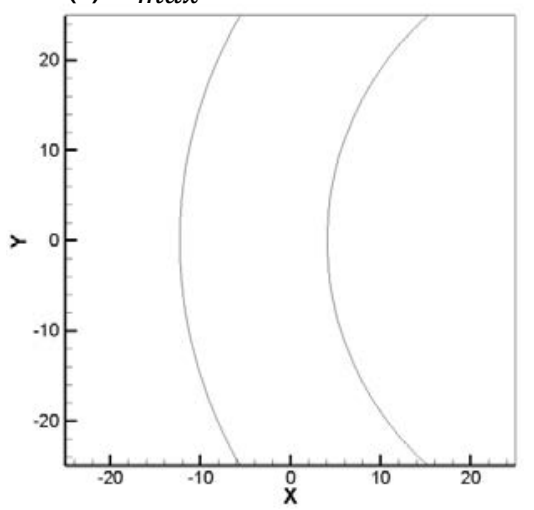

Figure 5.37. Pressure contours at values of \pm 0.001 for various $\sigma_{\max }$ and $\beta=2.0$ : $M_{x}=0.5$ and $M_{y}=0.0$ at $t=100$, with NPML $=25$. 

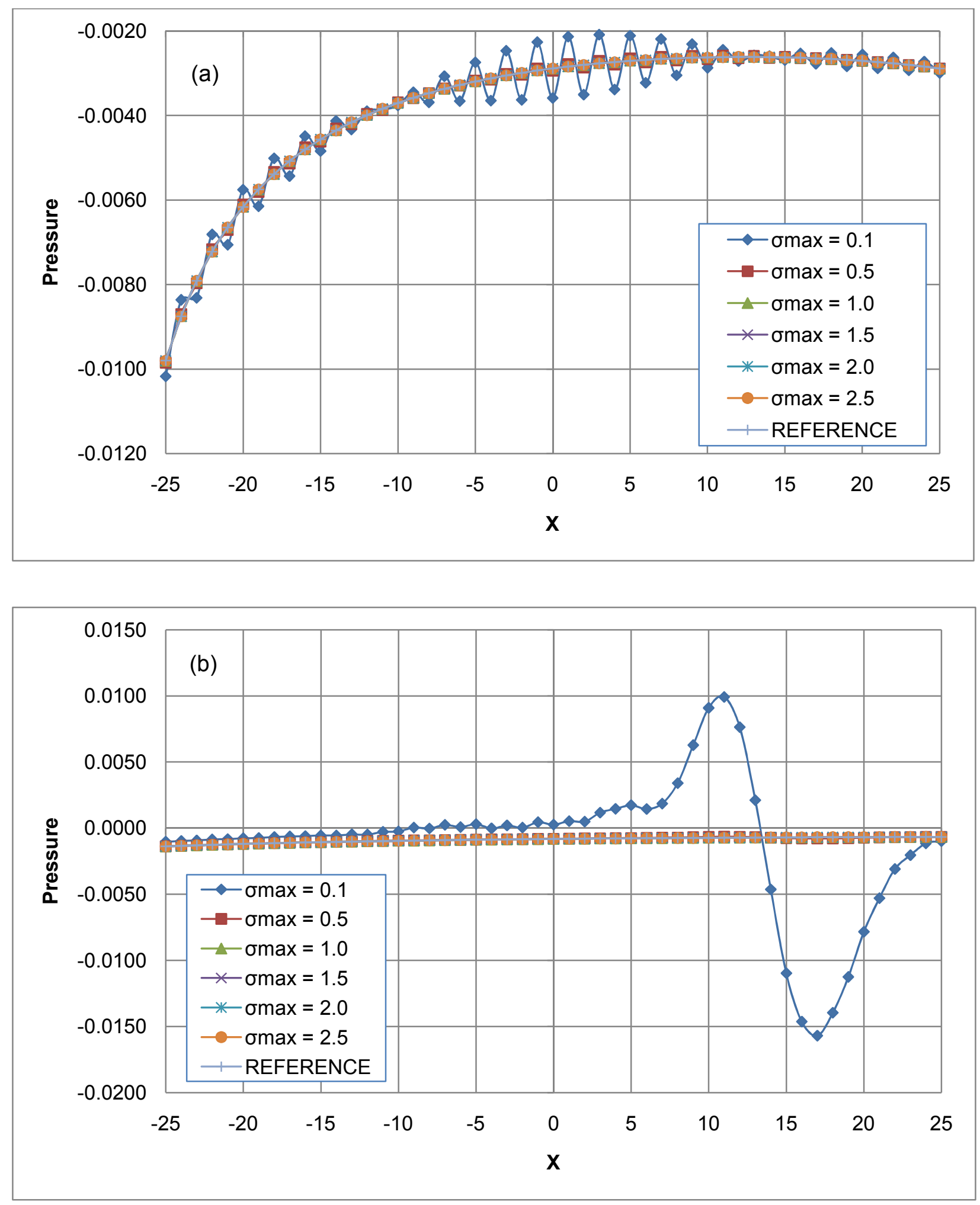

Figure 5.38. Pressure along $y=0$ line with PMLs of various $\sigma_{\max }$ and $\beta=2.0: M_{x}=$ 0.5 and $M_{y}=0.0$ at $(a) t=50$ and $(b) t=100$, with NPML $=25$. 


\subsubsection{Effect of Damping Rate}

Finally, the effect of the damping rate, $\beta$, the rate at which the damping is distributed inside the PML, was investigated. Here, NPML and $\sigma_{\max }$ were selected as 25 and 2.0, respectively. The value of $\beta$ was systematically changed, and computations were performed. A value of $\beta$ greater than one distributed the damping smoothly between the interior domain and the outer boundary of PML domain. The pressure contour plots at the time level $t=50$ is shown in Figure 5.23 for $\beta$ of $1.0,2.0,5.0,10.0,20.0$, and 50.0. From Figure 5.39, it is inferred that unlike NPML and $\sigma_{\max }, \beta$ has a range and within this range leads to an accurate solution. Thus, the rate at which the damping is distributed right from the Euler and PML domain interface towards the PML boundary had a serious impact on the effectiveness of the PML domain and therefore, the numerical solution. It is clearly seen in Figures 5.40(a) and (b), pressure along line $y=0$ at $t=50$ and $t=100$, respectively, that a higher value of $\beta$ results in waves with large oscillations. The solution was improved for values of $\beta$ above 1.0 but was contaminated for $\beta$ above 2.0. For values outside this range, the solution was not accurate. For the present case, an accurate solution was obtained for $\beta=2.0$ and this compares well with the reference solution, as shown in Figures 5.39 and 5.40(a) and (b). 
(a) $\beta=1.0$

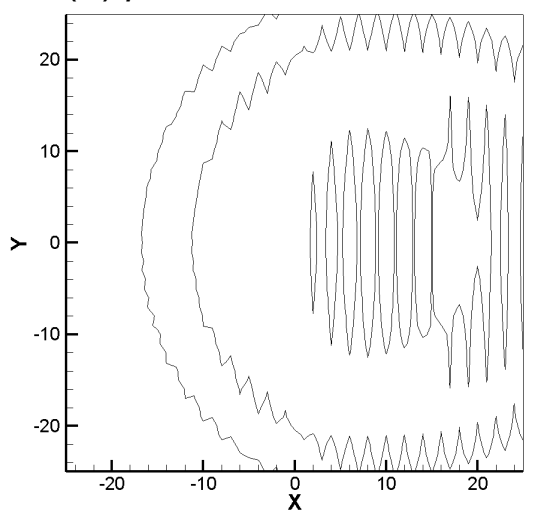

(c) $\beta=5.0$

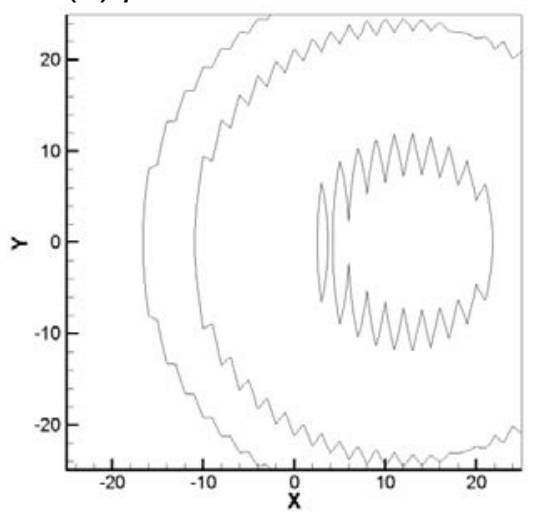

(e) $\beta=20.0$

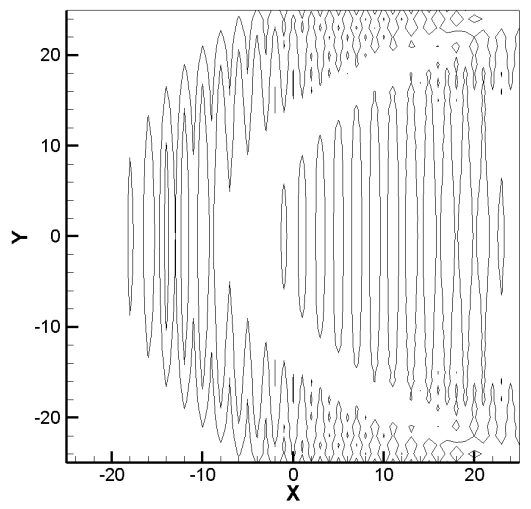

(b) $\beta=2.0$

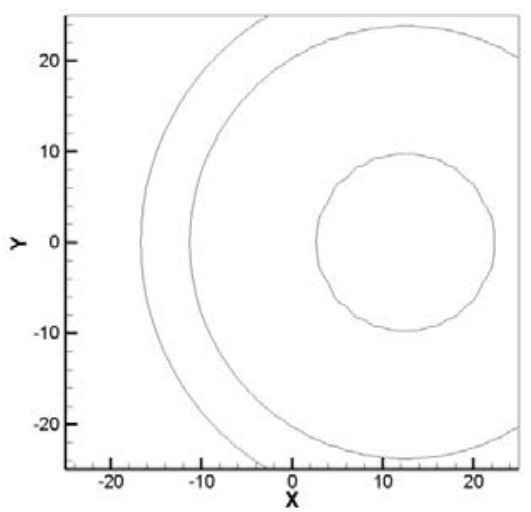

(d) $\beta=10.0$

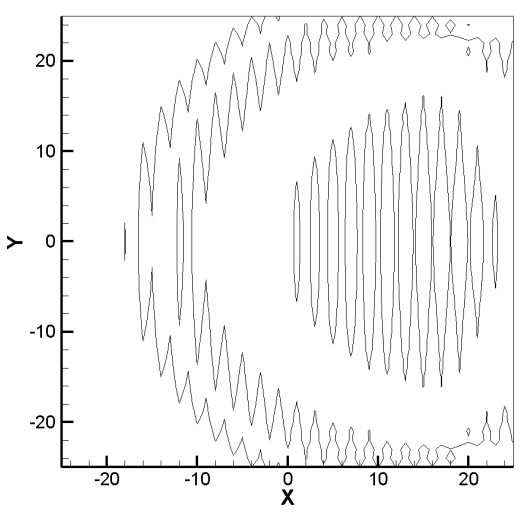

(f) $\beta=50.0$

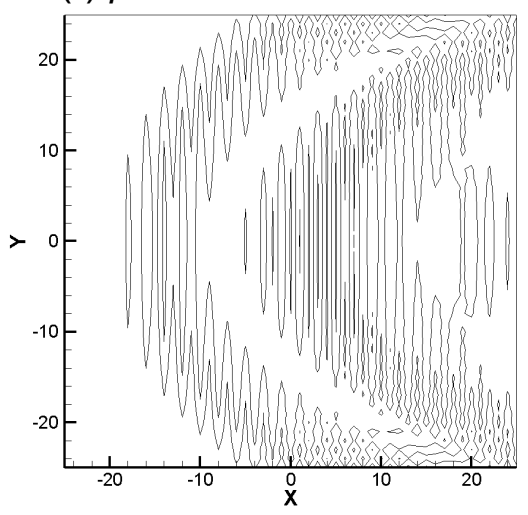

Figure 5.39. Pressure contours at values of \pm 0.005 for various $\beta$ and $\sigma_{\max }=2.0$ : $M_{x}=0.5$ and $M_{y}=0.0$ at $t=50$, with $\mathrm{NPML}=25$. 

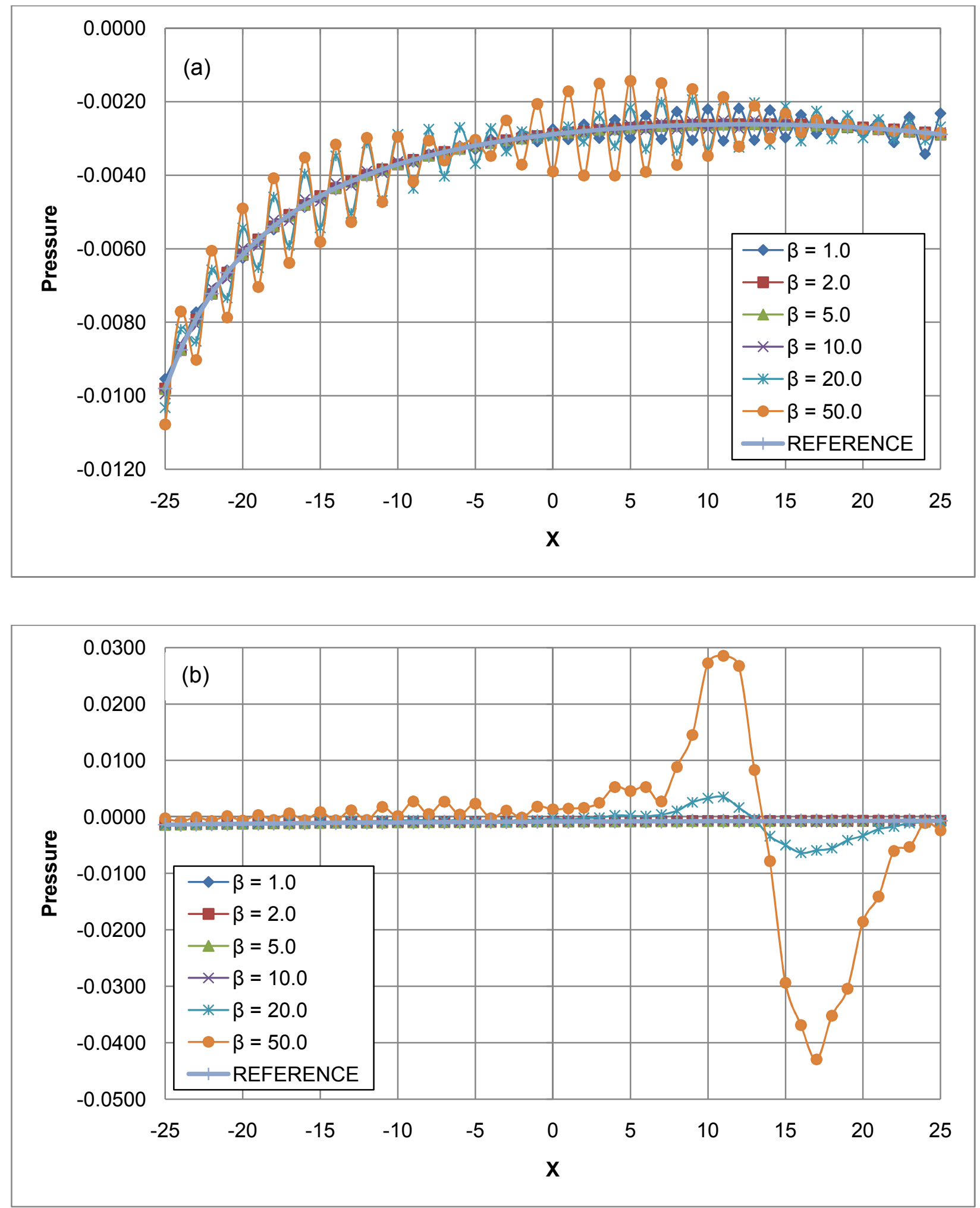

Figure 5.40. Pressure along $y=0$ line with PMLs of various $\beta$ and $\sigma_{\max }=2.0: M_{x}=$ 0.5 and $M_{y}=0.0$ at $(a) t=50$ and $(b) t=100$, with NPML $=25$. 


\section{CHAPTER 6}

\section{DIFFICULTIES ENCOUNTERED IN DEVELOPING PML FOR OBLIQUE MEAN FLOW}

\subsection{Two-Components PML Formulation}

In the previous sections, PML formulation was validated for both parallel flow and oblique mean flow. However, the validity of the formulation should be investigated for different combination of acoustic and vorticity pulses. Initially, the solution produced by the PML formulation for a given acoustic and vorticity pulse combination was considered. The linearized Euler equations support both acoustic and vorticity waves. Therefore, the formulation should be able to yield accurate results, subject to the initial conditions. To investigate this, the case used by Parrish and Hu [19] was selected. Conditions included an acoustic pulse located at the origin and three vorticity pulses positioned at $\left(x_{1}, y_{1}\right)=(25,25),\left(x_{2}, y_{2}\right)=\sqrt{2} / 2(25,25)$, and $\left(x_{3}, y_{3}\right)=(0,25)$. The amount of damping and the rate of damping distributed in the PML domain during the simulation were $\sigma_{\max }=2.0$ and $\beta=3.0$, respectively. The initial conditions were as follows:

$$
\begin{gathered}
\rho=\exp \left[(-\ln 2)\left(\frac{x^{2}+y^{2}}{16}\right)\right]+\sum_{n=1}^{3} \exp \left[(-\ln 2)\left(\frac{\left(x-x_{n}\right)^{2}+\left(y-y_{n}\right)^{2}}{16}\right)\right] \\
p=\exp \left[(-\ln 2)\left(\frac{x^{2}+y^{2}}{16}\right)\right] \\
u=\sum_{n=1}^{3}\left(y-y_{n}\right) \exp \left[(-\ln 2)\left(\frac{\left(x-x_{n}\right)^{2}+\left(y-y_{n}\right)^{2}}{16}\right)\right] \\
v=\sum_{n=1}^{3}\left(x-x_{n}\right) \exp \left[(-\ln 2)\left(\frac{\left(x-x_{n}\right)^{2}+\left(y-y_{n}\right)^{2}}{16}\right)\right]
\end{gathered}
$$


The computational domain was defined by [-60X60], [-60X60] which included ten PML layers added to four open boundaries of the interior domain. The objective here was to investigate the ability of the PML formulation and the unresolved issues associated with PML formulation in general. Before simulation was conducted for an oblique mean flow, it was performed for a flow parallel to the $x$-axis and another simulation for a flow parallel to the $y$-axis, where the parallel flow Mach number was 0.5 .

The density contours showing the acoustic and entropy pulses are shown in Figures 6.1 and 6.2 for a flow parallel to $x$ - axis and $y$-axis, respectively. The initial solution is shown in Figures 6.1(a) and (b), where the acoustic pulse at the origin and the three vorticity pulses can be observed. Figures 6.1(b) through (d) and Figures 6.2(a) through (d) show the waves in the Euler domain at (c) at $t=70, t=100$, and $t=200$, respectively. It can be seen that the waves leave the domain without causing any reflections at the boundary for the parallel flow cases at all the time levels shown. Furthermore, from the contour level values, it can be concluded from Figure 6.1(d) and Figure 6.2(d) that the perturbations decrease gradually as time goes increases. 
(a)

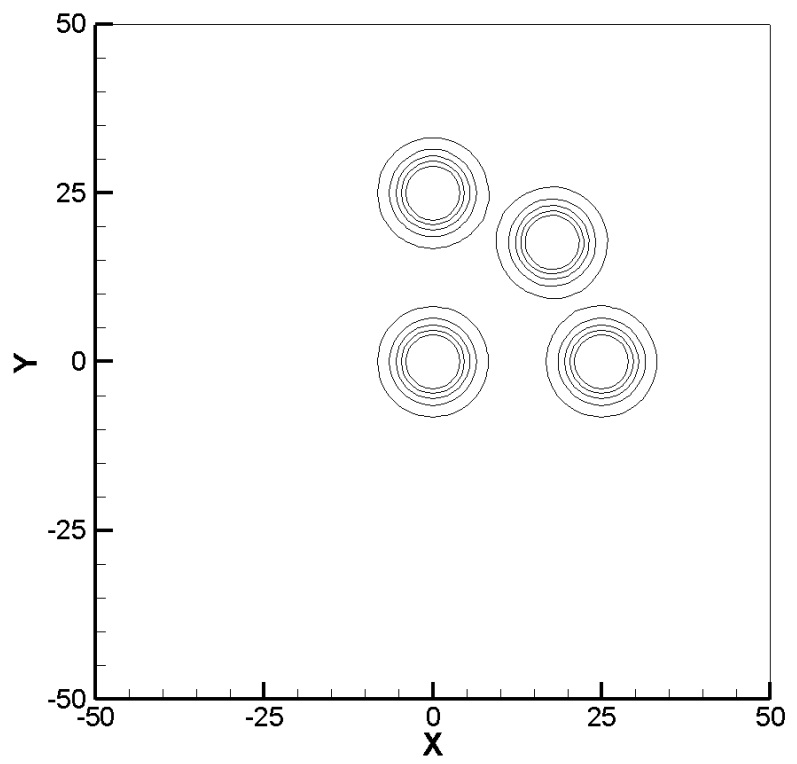

(c)

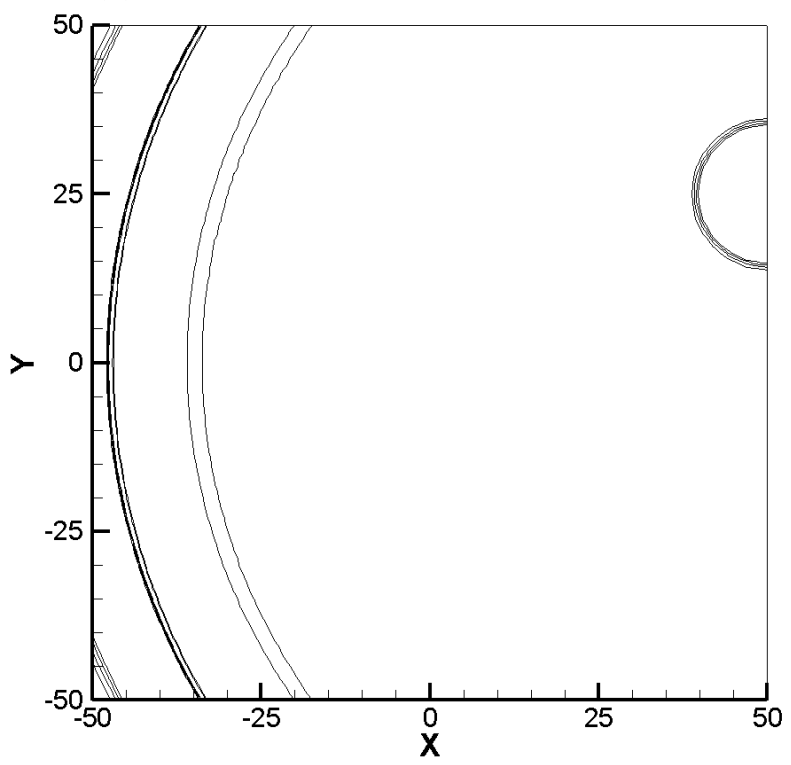

(b)

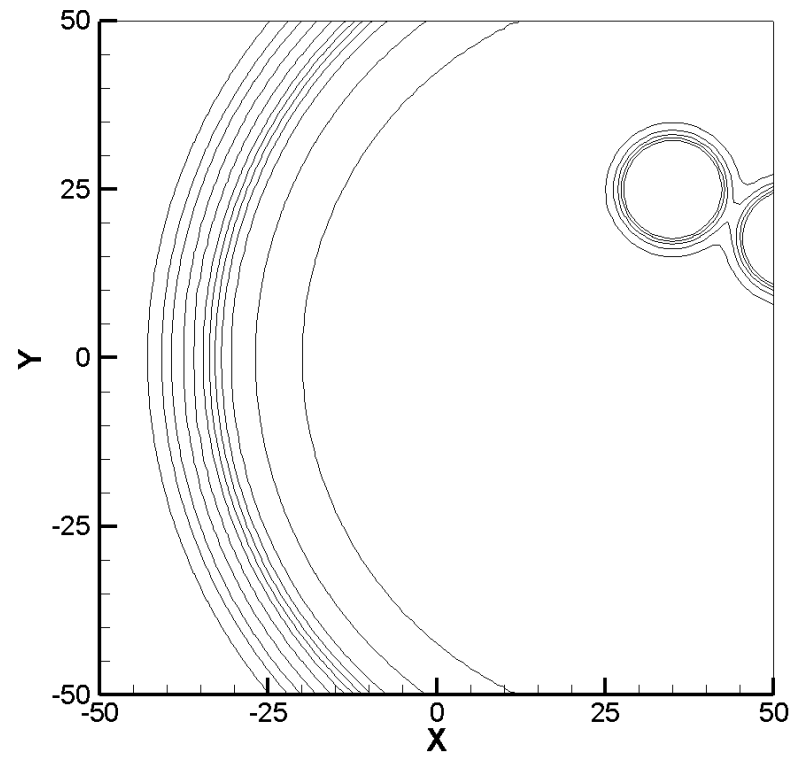

(d)

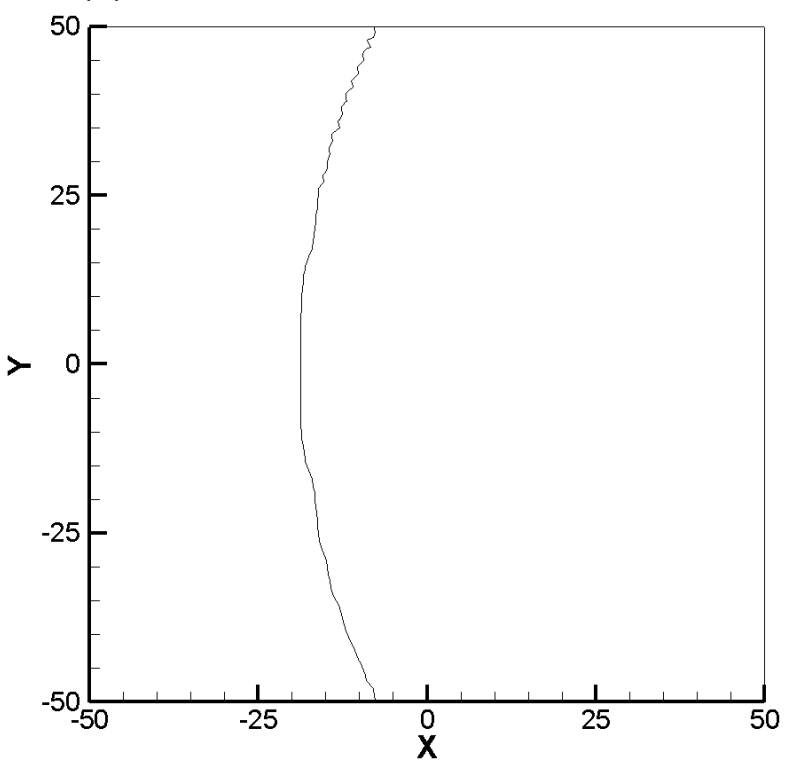

Figure 6.1. Density contours at values of $\pm 0.5, \pm 0.05, \pm 0.01$, and \pm 0.005 , showing acoustic and vorticity pulses: $M_{x}=0.5$ and $M_{y}=0.0$ at (a) $t=0,(b) t=70$, (c) $t=100$, and $(d) t=200$, with NPML $=10, \sigma_{\max }=2.0$, and $\beta=3.0$. 
(a)

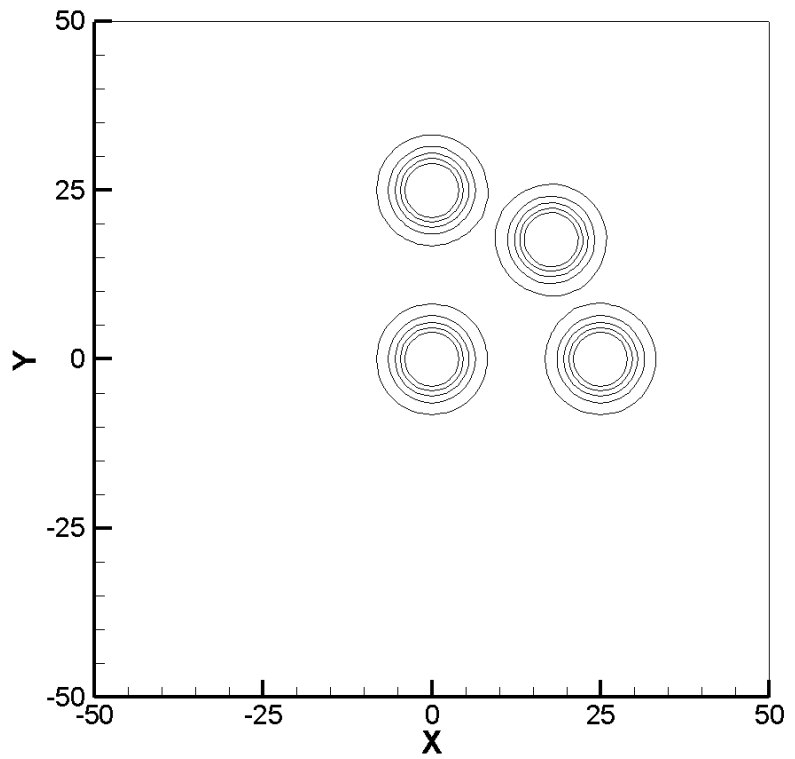

(c)

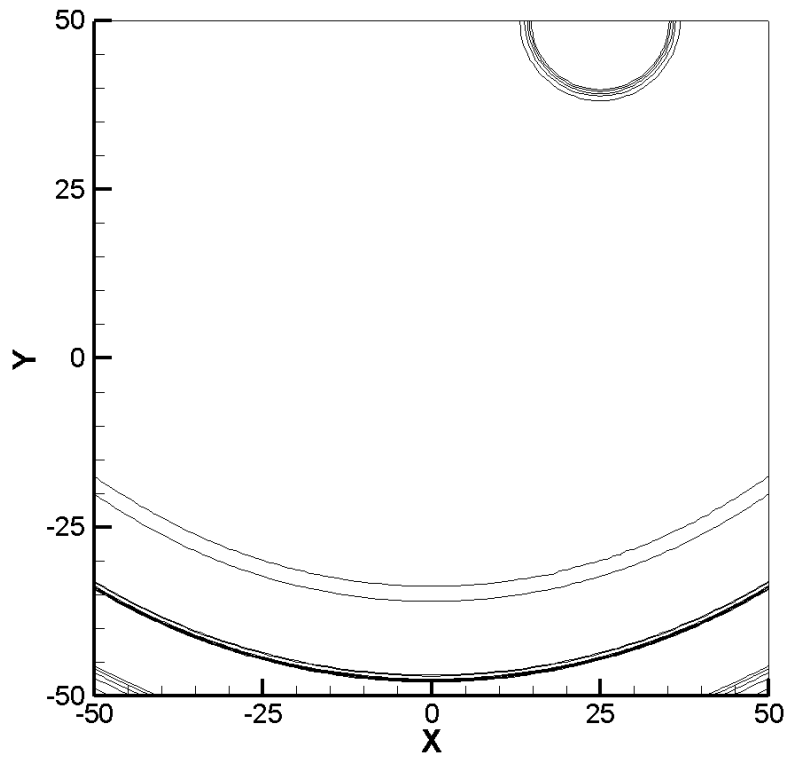

(b)

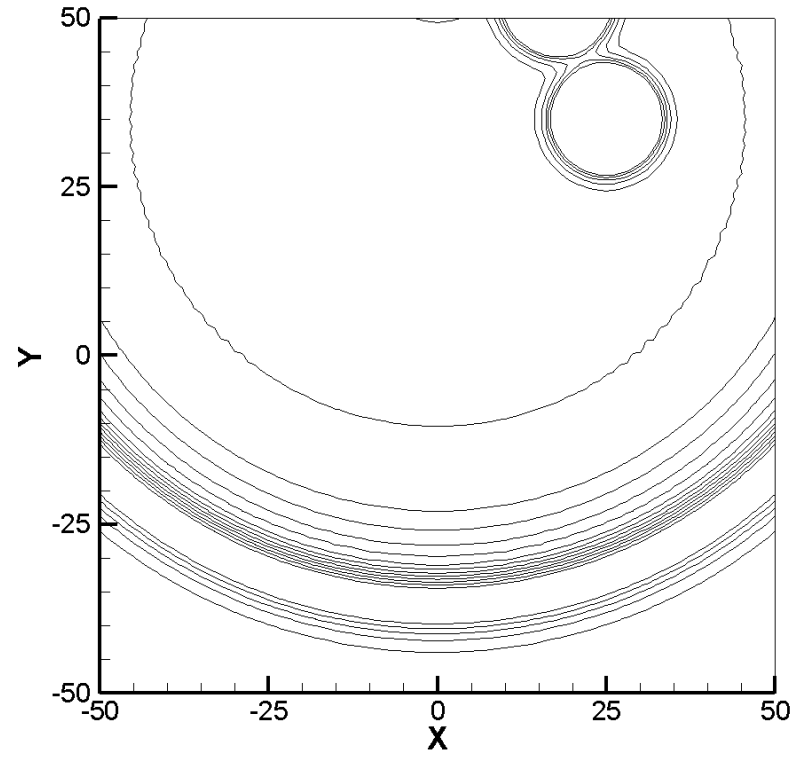

(d)

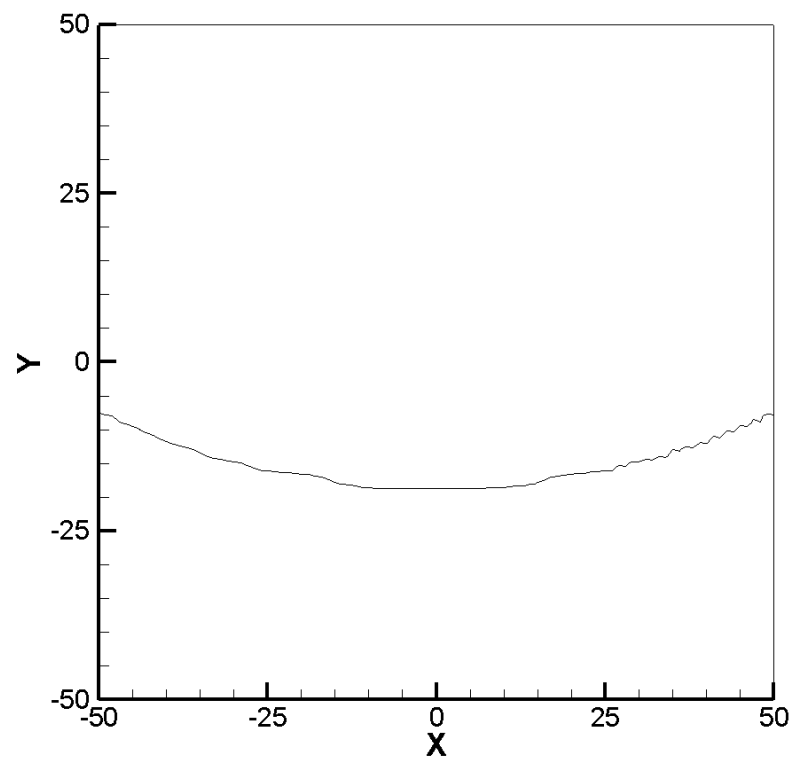

Figure 6.2. Density contours at values of $\pm 0.5, \pm 0.05, \pm 0.01$, and \pm 0.005 , showing acoustic and vorticity pulses: $M_{x}=0.0$ and $M_{y}=0.5$ at $(a) t=0,(b) t=70$, (c) $t=100$, and $(d) t=200$, with NPML $=10, \sigma_{\max }=2.0$, and $\beta=3.0$. 
Now, an oblique flow with the same initial conditions except the flow velocities was considered. In the oblique flow case, $M_{x}=M_{y}=0.5$. The density contours are presented in Figures 6.3(a) through 6.3(d). Indications of reflections coming back into the interior domain can be seen in Figure 6.3(b) at $t=70$. The penalty for this is a contaminated solution, which is quite evident in Figure 6.3(c). The complete toll taken by the reflected waves is seen in Figure 6.3(d), where the solution, at $t=200$, has values in the range of \pm 35.0 which explains undoubtedly that the perturbations did not vanish with time but increased with time, and that this solution is inaccurate and nonphysical.

It was suspected that the insufficient number of PML layers or damping quality of the PML may have caused these reflections. Simulations were performed with various PML thicknesses and a numerous combination of $\sigma_{\max }$ and $\beta$. Even the increase in the number of PML layers with increased damping did not result in an accurate solution. The contamination of the solution here was attributed to the reflections that occurred at the top right corner of the domain toward which the actual flow was defined for this problem.

According to $\mathrm{Hu}[10]$, it was found to be necessary to split $u$ and $v$ into three subcomponents when the mean flow is not parallel to any of the axes. The linearized Euler equations for an oblique mean flow and their corresponding PML equations proposed by Hu will be discussed in the next section. 
(a)

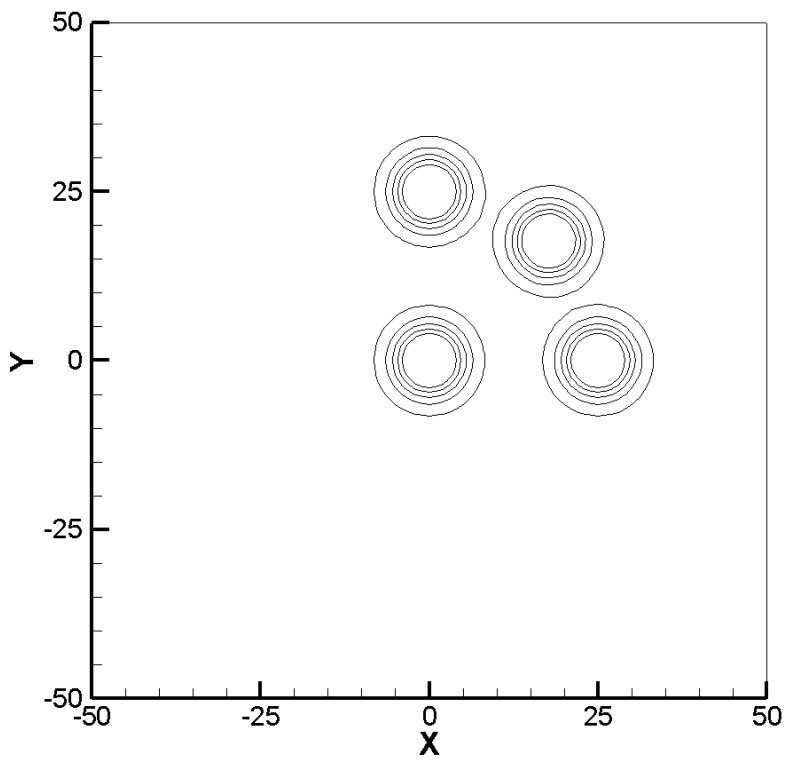

(c)

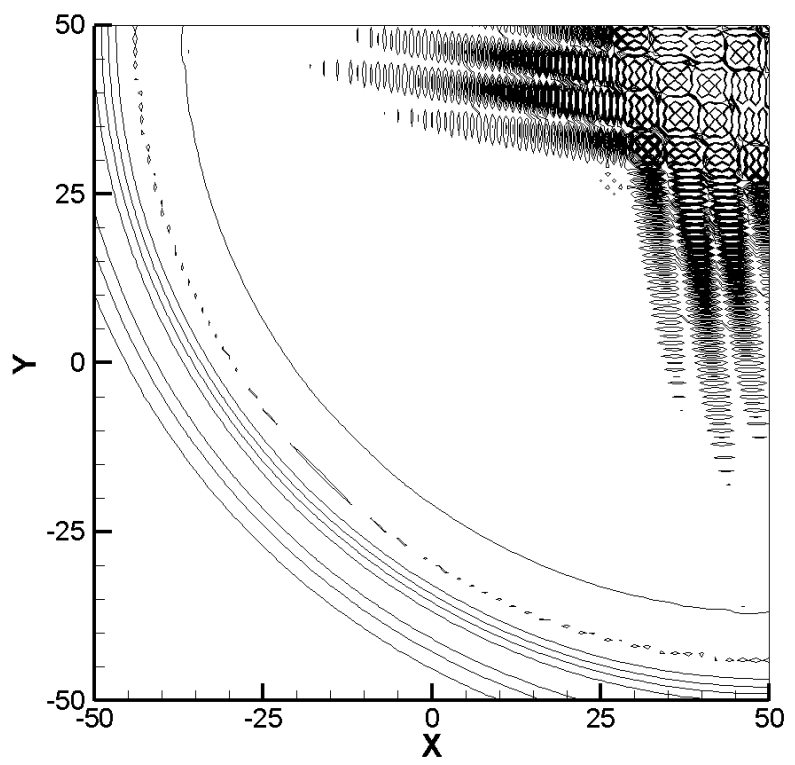

(b)

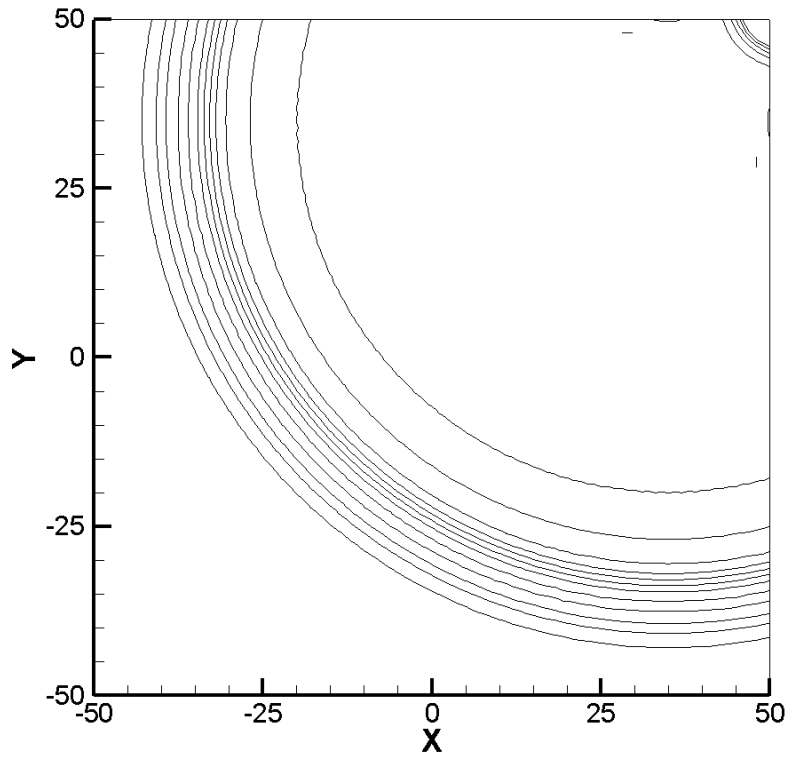

(d)

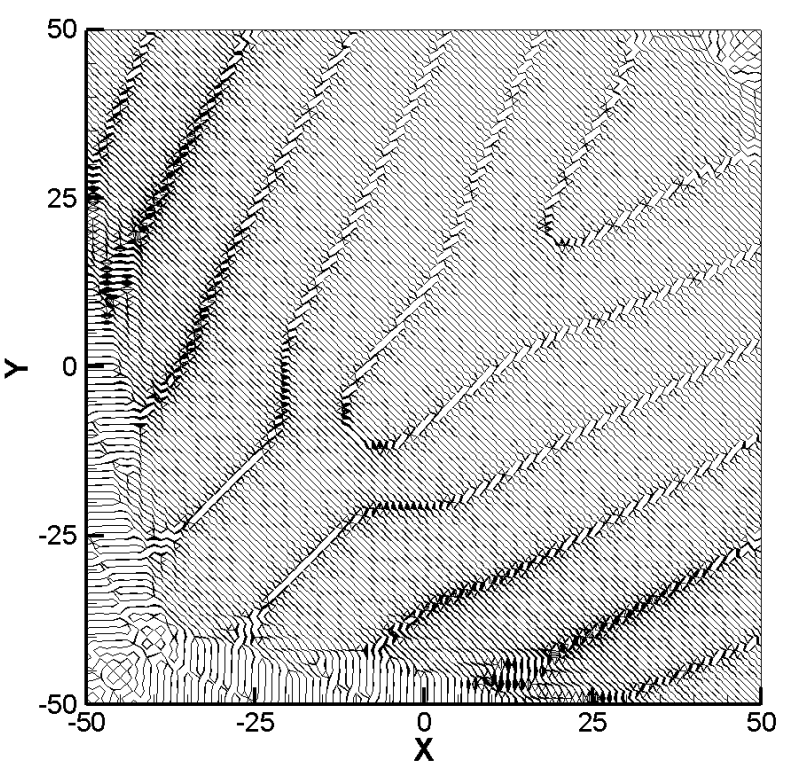

Figure 6.3. Density contours at values of \pm 0.5 and \pm 0.1 , showing acoustic and vorticity pulses: $M_{x}=0.5$ and $M_{y}=0.5$ at $(a) t=0,(b) t=70,(c) t=100$, and (d) $t=200$, with NPML $=10, \sigma_{\max }=2.0$, and $\beta=3.0$ 


\section{CHAPTER 7}

\section{DEVELOPMENT OF PML EQUATIONS USING THREE-COMPONENTS SPLITTING}

\subsection{PML Equations Using Three-Components Splitting}

For a mean flow not parallel to the $x$-axis, the linearized Euler equations are

$$
\begin{gathered}
\frac{\partial u}{\partial t}+M_{x} \frac{\partial u}{\partial x}+M_{y} \frac{\partial u}{\partial y}=-\frac{\partial p}{\partial x} \\
\frac{\partial v}{\partial t}+M_{x} \frac{\partial v}{\partial x}+M_{y} \frac{\partial v}{\partial y}=-\frac{\partial p}{\partial y} \\
\frac{\partial p}{\partial t}+M_{x} \frac{\partial p}{\partial x}+M_{y} \frac{\partial p}{\partial y}=-\left(\frac{\partial u}{\partial x}+\frac{\partial v}{\partial y}\right) \\
\frac{\partial \rho}{\partial t}+M_{x} \frac{\partial \rho}{\partial x}+M_{y} \frac{\partial \rho}{\partial y}=-\left(\frac{\partial u}{\partial x}+\frac{\partial v}{\partial y}\right)
\end{gathered}
$$

where $M_{x}$ and $M_{y}$ are the Mach numbers in the $x$ and $y$ directions, respectively. Hu found that it is necessary to split $u$ and $v$ into three sub-components when the flow is defined in an arbitrary direction [10].

Hu proposed the following PML equations:

$$
\begin{gathered}
\frac{\partial u_{1}}{\partial t}+\sigma_{x} u_{1}=-\frac{\partial\left(p_{1}+p_{2}\right)}{\partial x} \\
\frac{\partial u_{2}}{\partial t}+\sigma_{x} u_{2}=-M_{x} \frac{\partial\left(u_{1}+u_{2}+u_{3}\right)}{\partial x} \\
\frac{\partial u_{3}}{\partial t}+\sigma_{y} u_{3}=-M_{y} \frac{\partial\left(u_{1}+u_{2}+u_{3}\right)}{\partial y}
\end{gathered}
$$




$$
\begin{gathered}
\frac{\partial v_{1}}{\partial t}+\sigma_{y} v_{1}=-\frac{\partial\left(p_{1}+p_{2}\right)}{\partial y} \\
\frac{\partial v_{2}}{\partial t}+\sigma_{x} v_{2}=-M_{x} \frac{\partial\left(v_{1}+v_{2}+v_{3}\right)}{\partial x} \\
\frac{\partial v_{3}}{\partial t}+\sigma_{y} v_{3}=-M_{y} \frac{\partial\left(v_{1}+v_{2}+v_{3}\right)}{\partial y} \\
\frac{\partial p_{1}}{\partial t}+\sigma_{x} p_{1}=-\frac{\partial\left(u_{1}+u_{2}+u_{3}\right)}{\partial x}-M_{x} \frac{\partial\left(p_{1}+p_{2}\right)}{\partial x} \\
\frac{\partial p_{2}}{\partial t}+\sigma_{y} p_{2}=-\frac{\partial\left(v_{1}+v_{2}+v_{3}\right)}{\partial y}-M_{y} \frac{\partial\left(p_{1}+p_{2}\right)}{\partial y} \\
\frac{\partial \rho_{2}}{\partial t}+\sigma_{y} \rho_{2}=-\frac{\partial\left(v_{1}+v_{2}+v_{3}\right)}{\partial y}-M_{y} \frac{\partial\left(\rho_{1}+\rho_{2}\right)}{\partial y} \\
\frac{\partial t}{\partial t} \sigma_{x}=-\frac{\partial\left(u_{1}+u_{2}+u_{3}\right)}{\partial x}-M_{x} \frac{\partial\left(\rho_{1}+\rho_{2}\right)}{\partial x}
\end{gathered}
$$

It must be noted that when there is no mean flow, i.e., $M_{x}=M_{y}=0$, it is not necessary to split $u$ and $v$. This will result in six equations for the PML domain by deleting the second, third, fifth and sixth of the set of PML equations given by equations (7.5) to (7.14). The two-dimensional Euler equations, in Cartesian coordinates, written in the conservative form are

$$
\frac{\partial}{\partial t} Q+\frac{\partial}{\partial x} E+\frac{\partial}{\partial y} F+H=0
$$

where 


$$
Q=\left[\begin{array}{l}
\rho_{1} \\
\rho_{2} \\
u_{1} \\
u_{2} \\
u_{3} \\
v_{1} \\
v_{2} \\
v_{3} \\
p_{1} \\
p_{2}
\end{array}\right] ; E=\left[\begin{array}{l}
M_{x} \rho^{\prime}+u^{\prime} \\
0 \\
p^{\prime} \\
M_{x} u^{\prime} \\
0 \\
0 \\
M_{x} v^{\prime} \\
0 \\
M_{x} p^{\prime}+u^{\prime} \\
0
\end{array}\right] ; \quad F=\left[\begin{array}{l}
0 \\
M_{y} \rho^{\prime}+v^{\prime} \\
0 \\
0 \\
M_{y} u^{\prime} \\
p^{\prime} \\
0 \\
M_{y} v^{\prime} \\
0 \\
M_{y} p^{\prime}+v^{\prime}
\end{array}\right] ; H=\left[\begin{array}{l}
\sigma_{x} \rho_{1} \\
\sigma_{y} \rho_{2} \\
\sigma_{x} u_{1} \\
\sigma_{y} u_{2} \\
\sigma_{y} u_{3} \\
\sigma_{y} v_{1} \\
\sigma_{x} v_{2} \\
\sigma_{y} v_{3} \\
\sigma_{x} p_{1} \\
\sigma_{y} p_{2}
\end{array}\right]
$$

and

$$
\begin{gathered}
\rho^{\prime}=\rho_{1}+\rho_{2} \\
u^{\prime}=u_{1}+u_{2}+u_{3} \\
v^{\prime}=v_{1}+v_{2}+v_{3} \\
p^{\prime}=p_{1}+p_{2}
\end{gathered}
$$

\subsection{Numerical Investigation}

For the numerical testing with PML formulation of three-components splitting, the domain and initial conditions as used in section 6.1 were utilized. Thus, a computational domain of size [-60X60], [-60X60] with $N P M L$ of ten around the four open boundaries of the interior domain was selected. Figures $7.1(\mathrm{a})$ and (b) show the density contours at $t=70$ and $t=100$ for PML formulations with two-components and three-components splitting, respectively. In Figure 7.1(a), the solution appears to be reflection-free at $t=70$ when the computation is performed with three-components splitting. Reflections 
are clearly seen at the top right corner of the solution with two-components splitting. However, at $t=70$, Figure 7.1(b), even though the three-components splitting solution is better than the two-components splitting, the scheme did not prevent the waves from reflecting back into the solution. It can be seen that the three-components splitting works fairly well for small time durations but fails for a simulation with long time durations, such as the two-components splitting formulation.

Three-components splitting provides better results than two-components splitting for only a short time. The reflections in the case of the three-components splitting were less, but reflections were not completely prevented. When the simulation was conducted for a longer period of time, the waves were reflected back into the computational domain and contaminated the solution.

$\mathrm{Hu}$ [19] encountered a similar problem. However, in his case, an increase in the PML layer thickness reduced the error, and the error remained same for the large $t$ simulation. In the present case, the solution continued to diverge, with errors growing. Almost all investigators have experienced this reflection issue in one way or another, which in the literature is termed the corner effect in PML, and those investigators have had their own way of subduing the issue. Some have added damping functions, e.g., Tam [26], to eliminate the oscillations, and some have used numerical filtering, such as the one used by $\mathrm{Hu}$ [11]. Based on the work of Abarbanel et al. [27], Hu reiterated the importance of space-time transformation in his subsequent research papers. Thus, the analysis of the governing Euler equations for the dispersive waves has become an inevitable step in determining the space-time transformation. 
(a) Two - Components Splitting

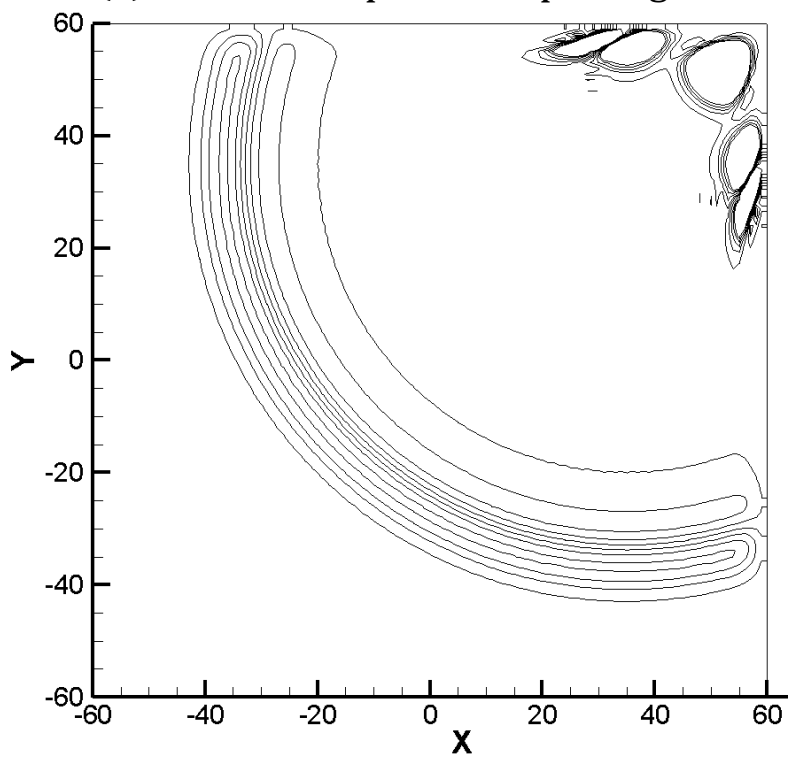

(b) Two - Components Splitting

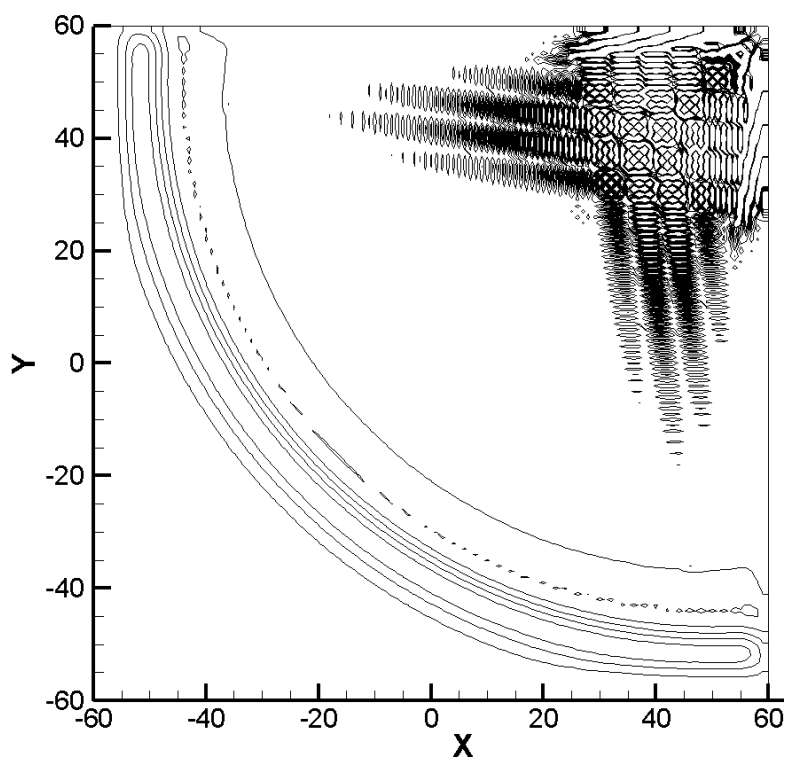

(a) Three - Components Splitting

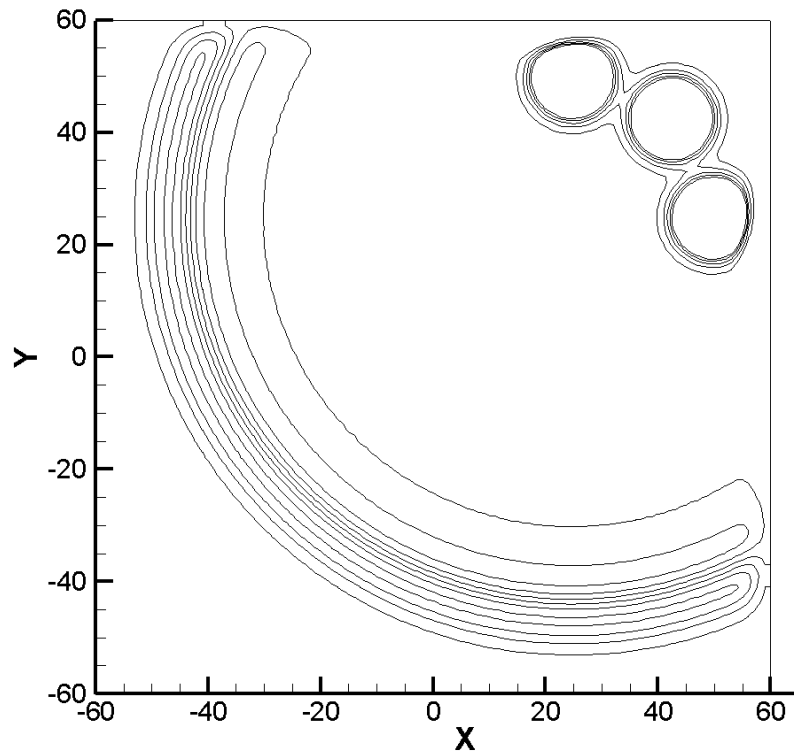

(b) Three - Components Splitting

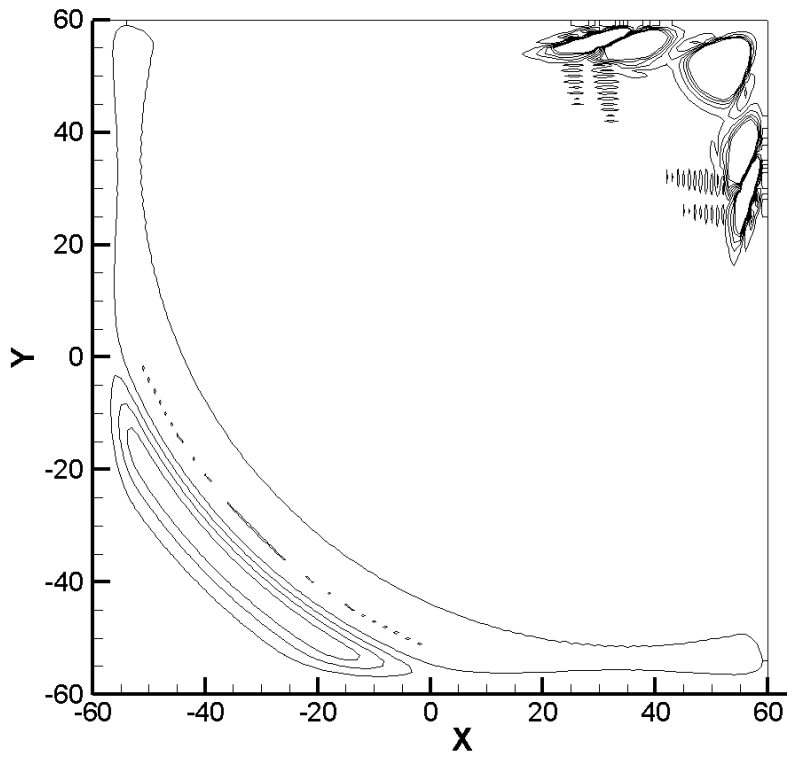

Figure 7.1. Density contours at values of \pm 0.1 , showing acoustic and vorticity pulses for $M_{x}=0.5$ and $M_{y}=0.5$ at $(a) t=70$ and $(b) t=100$, with NPML $=10, \sigma_{\max }=2.0$, and $\beta=3.0$.

This analysis must be carried out before constructing the PML formulation in the time domain. In his PML formulation for Euler equations with a non-uniform mean flow case [14], Hu showed that it is possible to determine the proper space-time transformation for the Euler equations with an arbitrary mean flow a priori, i.e., a unique value of the 
parameter $\beta$ that appears in his transformation equation can be determined through the dispersion analysis. 


\section{CHAPTER 8}

\section{CONCLUSIONS}

Any numerical scheme requires accurate treatment of boundary conditions for accurate results. These results should be obtained with reduced computational effort and cost. There are numerous schemes for treating boundaries. Absorbing boundary conditions being one of the popular areas of research inspired this current effort and began the idea of applying the popular non-reflecting boundary condition called perfectly matched layer along with existing numerical solvers to obtain accurate results with reduced computational cost. The main objective of the present work was to develop and implement a PML-absorbing boundary technique and investigate its effects on the numerical solution. In the process, it was felt necessary to have a clear understanding of the physics behind PML and its development, in order to utilize it in the numerical schemes.

In the present work, the PML equations were constructed in Cartesian coordinates for Euler equations linearized about a uniform mean flow. Since most of the physical problems are defined in an arbitrary shape, a coordinate transformation or mapping was required to solve the problem in the uniform domain. For this reason, PML equations were constructed in generalized coordinates. The formulation was validated for both uniform and non-uniform grid structures. The results obtained by this technique were compared with the simple extrapolation boundary treatment to distinguish the superiority of this kind of absorbing layer technique. It was demonstrated that the application of PML could help simulations with small truncated domains of actual unbounded problems, thus resulting in reduced computational effort. 
Furthermore, investigation of the effect of PML parameters on the solution of the numerical analysis was carried out, since there is no generalized procedure to estimate these parameters a priori. These parameters were approximately determined by using the empirical relation and refined by carrying out simulations where one variable was changed and the others were fixed. It was determined that these parameters may vary from one problem to another.

In the process of investigating the concept of PML, issues related to reflections at the corners in oblique mean flow cases were encountered. Simulations were carried out for uniform flow parallel to axes and flow in an arbitrary direction. PML construction based on both two-components and three-components splitting were carried out to study the corner effect. It was found that the latter scheme performed better than the former one. However, simulations performed for a long period of time revealed that error grew with time, which is undesirable for any numerical scheme. From the literature review, it was found that the reflections that arise from the corner when the mean flow is at an arbitrary direction can be attributed to the dispersive nature of the waves. Furthermore, a proper space-time transformation needs to be obtained using the analysis of dispersive waves supported by the governing equations, thus conserving the dispersion relation.

Although this work did not involve any dispersion analysis, efforts were made to gain knowledge in this area of PML construction. It was observed from the survey that investigators who claim successful PML formulation emphasize an appropriate spacetime transformation based on the dispersion analysis. Nevertheless, simulations carried out for a longer period of time resulted in reflections, and the construction of a perfectly 
matched layer with a reflection-free solution for long time simulations remains to be a challenge. 


\section{REFERENCES}




\section{REFERENCES}

[1] Klaus A. Hoffmann, Steve T. Chiang, "Computational Fluid Dynamics-Volume II" Engineering Education System, 2000.

[2] Dan Givoli, "Review Article - Non-reflecting Boundary Conditions", Journal of Computational Physics 94, 1-29, 1991.

[3] Sanjiva K. Lele, "Computational Aeroacoustics - A Review”, AIAA 97-0018.

[4] Ching Y. Loh, and Philip C. E. Jorgenson, "A Robust Absorbing Boundary Conditions for Compressible Flows", AIAA-2005-4716, 2005.

[5] Christopher K. W. Tam, "Advances in Numerical Boundary Conditions for Computational Aeroacoustics", AIAA-97-1774, 1997.

[6] M. Ehtesham Hayder, Fang Q. Hu, M. Yousuff Hussaini, "Towards Perfectly Absorbing Boundary Conditions For Euler Equations", ICASE Report No. 97-25, May 1997.

[7] Lakshmi S. Nizampatnam, "Investigation of Boundary Conditions for Computational Aeroacoustics", Thesis work for Master of science, Aerospace Engineering, Wichita State University, Kansas, 1999.

[8] Lakshmi S. Nizampatnam, J. Wong, Klaus A. Hoffmann, M. Papadakis, and R. K. Agarwal, "Development of Perfectly Matched Layer Boundary Conditions for Aeroacoustic Applications", AIAA-2000-0223, 2000.

[9] Jean-Pierre Berenger, "A Perfectly Matched Layer for the Absorption of Electromagnetic Waves," Journal of Computational Physics 114, 185-200, 1994.

[10] Fang Q. Hu, "On Absorbing Boundary Conditions for Linearized Euler Equations by a Perfectly Matched Layer", Journal of Computational Physics 129, 201-219, 1996.

[11] Fang Q. Hu, "On Perfectly Matched Layer as an Absorbing Boundary Condition", AIAA 96-1664, 1996.

[12] Fang Q. Hu, "A Stable, Perfectly Matched Layer for Linearized Euler Equations in Unsplit Physical Variables", Journal of Computational Physics 173, 455-480, 2001.

[13] Fang Q. Hu, "On Constructing Stable Perfectly Matched Layers as an Absorbing Boundary Conditions for Euler Equations", AIAA-2002-0227, 2002. 
REFERENCES (continued)

[14] Fang Q. Hu, "On Using Perfectly Matched Layer for the Euler Equations with a Non-Uniform Mean Flow", AIAA-2004-2966, 2004.

[15] Fang Q. Hu, "Absorbing Boundary Conditions", International Journal of Computational Fluid Dynamics, August 2004 Vol. 18 (6), pp. 513-522.

[16] Fang Q. Hu, "A Perfectly Matched Layer Absorbing Boundary Condition for Linearized Euler Equations With a Non-Uniform Mean Flow", Journal of Computational Physics 208, 469-492, 2005.

[17] Fang Q. Hu, X. D. Li, and D. K. Lin, "PML Absorbing Boundary Condition for Non-Linear Aeroacoustics Problems", AIAA-2006-2521, 2006.

[18] Fang Q. Hu, "On the Construction of PML Absorbing Boundary Condition for the Non-Linear Euler Equations", AIAA-2006-798, 2006.

[19] Sarah A. Parrish, and Fang Q. Hu, "Application of PML Absorbing Boundary Condition to Aeroacoustic Problems with an Oblique Mean Flow, AIAA-20073509, 2007.

[20] Fang Q. Hu, X. D. Li, D. K. Lin, “ Absorbing Boundary Conditions for Nonlinear Euler and Navier-Stokes Equations Based on the Perfectly Matched Layer Technique", Journal of Computational Physics 227, Issue 9, 4398-4424, 2008.

[21] D. K. Lin, X.D. Li, and Fang Q. Hu, "PML Absorbing Boundary Conditions for Nonlinear Euler Equations in Primitive Variables", AIAA-2009-0006, 2009.

[22] John W. Goodrich, Thomas Hagstrom, "A Comparison of Two Accurate Boundary Treatments for Computational Aeroacoustics”, AIAA-1997-1585, 1997.

[23] Saul Aberbanel, and David Gottlieb, "A Mathematical Analysis of the PML Method", Journal of Computational Physics 134, Issue 2, 357-363, 1997.

[24] Adib N. Rahmouni, "An Algebraic Method to Develop Well-Posed PML Models: Absorbing Layers, Perfectly Matched Layers, Linearized Euler Equations", Journal of Computational Physics 197, Issue 1, 99-115, 2004.

[25] J. S. Hesthaven, "On the Analysis and Construction of Perfectly Matched Layers for the Linearized Euler Equations", Journal of Computational Physics 142, Issue 1, 129-147, 1998.

[26] Christopher K.W. Tam, and Laurent Auriault, "Perfectly Matched Layer as Absorbing Boundary Condition for the Linearized Euler Equations in Open and Ducted Domains", Journal of Computational Physics 144, 213-234, 1998. 
REFERENCES (continued)

[27] S. Abarbanel, D. Gottlieb, and J. S. Hesthaven, "Well-Posed Perfectly Matched Layers for Advective Acoustics", Journal of Computational Physics 154, Issue 2, 266-283, 1999.

[28] Thomas Hagstrom, and Igor Nazarov, "Absorbing Layers and Radiation Boundary Conditions for Jet Flow Simulations", AIAA-2002-2606, 2002.

[29] Thomas Hagstrom, and Igor Nazarov, "Absorbing Layers and Radiation Boundary Conditions for Shear Flow Calculations”, AIAA-2003-3298, 2003.

[30] Jean-François Dietiker, Klaus A. Hoffmann, and Michael Papadakis, "Development of Three-Dimensional PML Boundary Conditions for Aeroacoustics Applications", AIAA-2001-2790, 2001.

[31] Mirela Caraeni, and Laszlo Fuchs, "Investigation of Nonreflective Boundary conditions for Computational Aeroacoustics", AIAA Journal, Vol. 44, No. 9, September 2006.

[32] Klaus A. Hoffmann, Steve T. Chiang, "computational Fluid Dynamics - Volume I", Engineering Education System, 2000.

[33] J. C. Hardin, J. R. Ristorcelli, and C. K. W. Tam, "ICASE/LaRC Workshop on Benchmark Problems in Computational Aeroacoustics", NASA Conference Publication 3300, May 1995. 


\section{APPENDIX}




\section{APPENDIX}

GOVERNING EQUATIONS IN TERMS OF PERTURBATION VARIABLES

\section{Euler Equation in Cartesian Form}

The governing differential equations in a vector form for the conservation laws for an inviscid flow (Euler equations) in Cartesian coordinate system is

$$
\frac{\partial}{\partial t} Q+\frac{\partial}{\partial x} F+\frac{\partial}{\partial y} G=0
$$

Where the unknown vector $Q$ and inviscid flux vectors are

$$
\begin{gathered}
Q=\left[\begin{array}{c}
\rho \\
\rho u \\
\rho v \\
\rho e_{t}
\end{array}\right] ; F=\left[\begin{array}{c}
\rho u \\
\rho u^{2}+p \\
\rho u v \\
\left(\rho e_{t}+p\right) u
\end{array}\right] ; G=\left[\begin{array}{c}
\rho v \\
\rho v u \\
\rho v^{2}+p \\
\left(\rho e_{t}+p\right) v
\end{array}\right] \\
e_{t}=e_{i}+\frac{1}{2}\left(u^{2}+v^{2}\right) \\
h=e_{i}+\frac{p}{\rho}
\end{gathered}
$$

where $e_{t}, e_{i}$, and $h$ are the total energy, internal energy and enthalpy.

The steady mean flow equations are;

$$
\begin{gathered}
\frac{\partial}{\partial x} \bar{F}+\frac{\partial}{\partial y} \bar{G}=0 \\
\bar{F}=\left[\begin{array}{c}
\bar{\rho} \bar{u} \\
\bar{\rho} \bar{u}^{2}+\bar{p} \\
\bar{\rho}-\bar{v} \\
\left(\bar{\rho} \bar{e}_{t}+\bar{p}\right) \bar{u}
\end{array}\right] ; \bar{G}=\left[\begin{array}{c}
\bar{\rho} \bar{v} \\
\bar{\rho} \bar{v} \\
\bar{\rho}-2 \\
(\bar{\rho}-\bar{p} \\
\left.\overline{e_{t}}+\bar{p}\right) \bar{v}
\end{array}\right]
\end{gathered}
$$




\section{APPENDIX (continued)}

where $\bar{u}, \bar{v}$, are the mean velocities in $x$ and $y$ directions, respectively; $\bar{p}$, and $\bar{\rho}$ are, pressure and density, respectively. The dependent variables of unsteady Euler equations and the steady mean flow equations are non-dimensionalized using the uniform mean flow conditions. The velocities are non-dimensionalized by the speed of sound $a_{0}$, the density by $\bar{\rho}_{0}$ and the pressure by $\bar{\rho}_{0} a_{0}^{2}$, where $\bar{\rho}_{0}$ is the uniform mean density. The non-dimensionalization is carried out as follows;

$$
\begin{gathered}
\rho^{*}=\frac{\rho}{\bar{\rho}_{0}} ; \bar{\rho}^{*}=\frac{\bar{\rho}}{\bar{\rho}_{0}} ; u^{*}=\frac{u}{a_{0}} ; \bar{u}^{*}=\frac{\bar{u}}{a_{0}} ; v^{*}=\frac{v}{a_{0}} ; \bar{v}^{*}=\frac{\bar{v}}{a_{0}} ; \\
t^{*}=\frac{t a_{0}}{L} ; x^{*}=\frac{x}{L} ; y^{*}=\frac{y}{L} ; p^{*}=\frac{p}{\bar{\rho}_{0} a_{0}^{2}} ; \bar{p}^{*}=\frac{\bar{p}}{\bar{\rho}_{0} a_{0}^{2}}
\end{gathered}
$$

The first-order perturbation equations are derived from the assumption;

$$
\text { Actual quantity = Steady mean quantity }+ \text { Time varying acoustic quantity }
$$

i.e.,

$$
\begin{gathered}
u=\bar{u}+u^{\prime} \\
v=\bar{v}+v^{\prime} \\
\rho=\bar{\rho}+\rho^{\prime} \\
p=\bar{p}+p^{\prime}
\end{gathered}
$$

Therefore,

$$
\text { Perturbation quantity = Actual quantity }- \text { Steady mean quantity }
$$

The resulting first-order perturbation equation, obtained from equation (A-1.6), is given by 


\section{APPENDIX (continued)}

$$
\frac{\partial}{\partial t} Q+\frac{\partial}{\partial x} F+\frac{\partial}{\partial y} G=0
$$

Where ' $Q$ ', ' $F$ ', and ' $G$ ' are provided by equations (A-1.8) and (A-1.9).

$$
\begin{aligned}
& Q=\left[\begin{array}{c}
(\rho)-(\bar{\rho}) \\
(\rho u)-(\bar{\rho} \bar{u}) \\
(\rho v)-(\bar{\rho} v) \\
\left(\rho e_{t}\right)-\left(\bar{\rho} \bar{e}_{t}\right)
\end{array}\right] ; \quad F=\left[\begin{array}{c}
(\rho u)-(\overline{\rho u}) \\
\left(\rho u^{2}+p\right)-\left(\overline{\rho u}{ }^{2}+\bar{p}\right) \\
(\rho u v)-(\bar{\rho} \overline{u v}) \\
\left(\left(\rho e_{t}+p\right) u\right)-\left(\left(\bar{\rho}_{t}+\bar{p}\right) \bar{u}\right)
\end{array}\right] \\
& G=\left[\begin{array}{c}
(\rho v)-(\bar{\rho} \bar{v}) \\
(\rho v u)-(\bar{\rho}-\bar{u}) \\
\left(\rho v^{2}+p\right)-\left(\bar{\rho} \bar{v}^{2}+\bar{p}\right) \\
\left(\left(\rho e_{t}+p\right) v\right)-\left(\left(\bar{\rho} \bar{e}_{t}+\bar{p}\right) \bar{v}\right)
\end{array}\right]
\end{aligned}
$$

which can be evaluated as

$$
Q=\left[\begin{array}{c}
\rho^{\prime} \\
(\rho u)^{\Delta} \\
(\rho v)^{\Delta} \\
(\rho e)^{\Delta}
\end{array}\right] ; F=\left[\begin{array}{c}
(\rho u)^{\Delta} \\
\bar{u}\left[2(\rho u)^{\Delta}-\bar{u} \rho^{\prime}\right]+p^{\prime} \\
\bar{v}\left[(\rho u)^{\Delta}-\bar{u} \rho^{\prime}\right]+(\rho v)^{\Delta} \bar{u} \\
\bar{u}\left[(\rho h)^{\Delta}-\bar{h} \rho^{\prime}\right]+(\rho u)^{\Delta} \bar{h}
\end{array}\right] ; G=\left[\begin{array}{c}
(\rho v)^{\Delta} \\
\bar{u}\left[(\rho v)^{\Delta}-\bar{v} \rho^{\prime}\right]+(\rho u)^{\Delta} \bar{v} \\
\bar{v}\left[2(\rho v)^{\Delta}-\bar{v} \rho^{\prime}\right]+p^{\prime} \\
\bar{u}\left[(\rho h)^{\Delta}-\bar{h} \rho^{\prime}\right]+(\rho v)^{\Delta} \bar{h}
\end{array}\right]
$$

The symbol " $\Delta$ " represents the difference between the actual quantity and the steady mean flow quantity. The details of the evaluation of the elements of the flux terms are given in the subsequent paragraphs. The first element of ' $Q$ ' is calculated using (A-1.6) as;

$$
\rho-\bar{\rho}=\rho^{\prime}
$$




\section{APPENDIX (continued)}

The second term is evaluated as;

$$
\begin{aligned}
\rho u-\bar{\rho} \bar{u} & =\left(\bar{\rho}+\rho^{\prime}\right)\left(\bar{u}+u^{\prime}\right)-\bar{\rho} \bar{u} \\
& =\bar{\rho} u+\bar{\rho} u^{\prime}+\rho^{\prime} \bar{u}+\rho^{\prime} u^{\prime}-\bar{\rho} u \\
& =\bar{\rho} u^{\prime}+\rho^{\prime} \bar{u} \\
& =(\rho u)^{\Delta}
\end{aligned}
$$

The perturbation quantities are small and therefore, their product is neglected. Similarly, the third and fourth terms are;

$$
\begin{gathered}
\rho v-\bar{\rho} \bar{v}=\bar{\rho} v^{\prime}+\rho^{\prime} \bar{v}=(\rho v)^{\Delta} \\
\rho e_{t}-\bar{\rho} e_{t}=\frac{p^{\prime}}{\gamma-1}+\bar{\rho} \bar{u} u^{\prime}+\bar{\rho}-v^{\prime}+\frac{1}{2} \rho^{\prime}\left(\bar{u}^{2}+\bar{v}^{2}\right)=(\rho e)^{\Delta}
\end{gathered}
$$

where

$$
p^{\prime}=(\gamma-1)\left\{(\rho e)^{\Delta}-\frac{1}{2}\left[\bar{u}\left(2(\rho u)^{\Delta}-\bar{u} \rho^{\prime}\right)+\bar{v}\left(2(\rho v)^{\Delta}-\bar{v} \rho^{\prime}\right)\right]\right\}
$$

Another expression that may be used in the derivation is;

$$
\rho h-\bar{\rho} \bar{h}=(\rho e)^{\Delta}+p^{\prime}=(\rho h)^{\Delta}
$$

The second element of ' $F$ ' is derived as;

$$
\begin{aligned}
\left(\rho u^{2}+p\right)-\left(\bar{\rho} \bar{u}^{2}+\bar{p}\right)= & {\left[\left(\bar{\rho}+\rho^{\prime}\right)\left(\bar{u}+u^{\prime}\right)^{2}\right]+\left(\bar{p}+p^{\prime}\right)-\bar{\rho} \bar{u}^{2}-\bar{p} } \\
& =\left[\left(\bar{\rho}+\rho^{\prime}\right)\left(\bar{u}^{2}+u^{\prime 2}+2 \bar{u} u^{\prime}\right)\right]+\bar{p}+p^{\prime}-\bar{\rho} \bar{u}^{2}-p_{0} \\
& =\bar{\rho} \bar{u}^{2}+\bar{\rho} u^{\prime 2}+2 \bar{\rho} \bar{u} u^{\prime}+\rho^{\prime} \bar{u}^{2}+\rho^{\prime} u^{\prime 2}+2 \rho^{\prime} \bar{u} u^{\prime}-\bar{\rho} \bar{u}^{2}+p^{\prime} \\
& =\bar{u}\left[2 \bar{\rho} u^{\prime}+2 \rho^{\prime} u^{\prime}+\rho^{\prime} \bar{u}\right]+\bar{\rho} u^{\prime 2}+\rho^{\prime} u^{\prime 2}+p^{\prime}
\end{aligned}
$$




\section{APPENDIX (continued)}

$$
\begin{aligned}
& =\bar{u}\left[2 \bar{\rho} u^{\prime}+\rho^{\prime} \bar{u}+\rho^{\prime} \bar{u}-\rho^{\prime} \bar{u}+2 \rho^{\prime} u^{\prime}\right]+\bar{\rho} u^{\prime 2}+\rho^{\prime} u^{\prime 2}+p^{\prime} \\
& =\bar{u}\left[2\left[\bar{\rho} u^{\prime}+\rho^{\prime} \bar{u}\right]-\rho^{\prime} \bar{u}+2 \rho^{\prime} u^{\prime}\right]+\bar{\rho} u^{\prime 2}+\rho^{\prime} u^{\prime 2}+p^{\prime} \\
& =\bar{u}\left[2(\rho u)^{\Delta}-\bar{u} \rho^{\prime}\right]+2 \rho^{\prime} u^{\prime} \bar{u}+\bar{\rho} u^{\prime 2}+p^{\prime} \\
& =\bar{u}\left[2(\rho u)^{\Delta}-\bar{u} \rho^{\prime}\right]+p^{\prime}
\end{aligned}
$$

where the products of perturbation quantities have been neglected.

Similarly, the remaining terms of ' $F$ ' are determined to be;

$$
\begin{aligned}
& (\rho u v)-(\overline{\rho u v})=\bar{v}\left[(\rho u)^{\Delta}-\bar{u} \rho^{\prime}\right]+(\rho v)^{\Delta} \bar{u} \\
& (\rho u h)-(\overline{\rho u} \bar{h})=\bar{u}\left[(\rho h)^{\Delta}-\bar{h} \rho^{\prime}\right]+(\rho u)^{\Delta} \bar{h}
\end{aligned}
$$

and the elements of ' $G$ ' are estimated in the same fashion.

\section{Continuity Equation for Non-Uniform Mean Flow}

From equations $(A-1.7)$ and $(A-1.8)$, the system of equations governing the flow is written. The Continuity equation is written as;

$$
\frac{\partial \rho}{\partial t}+\frac{\partial(\rho u)}{\partial x}+\frac{\partial(\rho v)}{\partial y}=0
$$

Using equation (A-1.6), the continuity equation in perturbation quantities is thus written as,

$$
\frac{\partial(\rho-\bar{\rho})}{\partial t}+\frac{\partial(\rho u-\bar{\rho} u)}{\partial x}+\frac{\partial(\rho v-\bar{\rho} \bar{v})}{\partial y}=0
$$




\section{APPENDIX (continued)}

In non-dimensional form;

$$
\begin{gathered}
\frac{\partial\left(\rho^{*} \bar{\rho}_{0}-\bar{\rho}^{*} \bar{\rho}_{0}\right)}{\partial\left(\frac{t^{*} L}{a_{0}}\right)}+\frac{\partial\left(\rho^{*} \bar{\rho}_{0} u^{*} a_{0}-\bar{\rho}^{*} \bar{\rho}_{0} \bar{u}^{*} a_{0}\right)}{\partial\left(x^{*} L\right)}+\frac{\partial\left(\rho^{*} \bar{\rho}_{0} v^{*} a_{0}-\bar{\rho}^{*} \bar{\rho}_{0} \bar{v}^{*} a_{0}\right)}{\partial\left(y^{*} L\right)}=0 \\
\frac{\bar{\rho}_{0} a_{0}}{L}\left[\frac{\partial\left(\rho^{*}-\bar{\rho}^{*}\right)}{\partial t^{*}}+\frac{\partial\left(\rho^{*} u^{*}-\bar{\rho}^{*} u-u^{*}\right)}{\partial x^{*}}+\frac{\partial\left(\rho^{*} v^{*}-\bar{\rho}^{*}-*\right)}{\partial y^{*}}\right]=0 \\
\frac{\partial\left(\rho^{*}-\bar{\rho}^{*}\right)}{\partial t^{*}}+\frac{\partial\left(\rho^{*} u^{*}-\bar{\rho}^{*} \bar{u}\right)}{\partial x^{*}}+\frac{\partial\left(\rho^{*} v^{*}-\bar{\rho}^{*} \bar{v}^{*}\right)}{\partial y^{*}}=0
\end{gathered}
$$

Using equation (A-1.6), it can be written that,

$$
\begin{gathered}
\frac{\partial \rho^{\prime}}{\partial t^{*}}+\frac{\partial\left(\bar{\rho}^{*} u^{\prime}+\rho^{\prime^{\prime}} \bar{u}^{*}\right)}{\partial x^{*}}+\frac{\partial\left(\bar{\rho}^{*} v^{\prime}+\rho^{\rho^{-*}} v^{*}\right)}{\partial y^{*}}=0 \\
\frac{\partial \rho^{\prime}}{\partial t^{*}}+\bar{\rho}^{*} \frac{\partial u^{\prime}}{\partial x^{*}}+u^{\prime} \frac{\partial \bar{\rho}^{*}}{\partial x^{*}}+\rho^{\prime} \frac{\partial \bar{u}^{*}}{\partial x^{*}}+\bar{u}^{*} \frac{\partial \rho^{\prime}}{\partial x^{*}}+\bar{\rho}^{*} \frac{\partial v^{\prime}}{\partial y^{*}}+v^{\prime} \frac{\partial \bar{\rho}^{*}}{\partial y^{*}}+\rho^{\prime} \frac{\partial \bar{v}^{*}}{\partial y^{*}}+\bar{v}^{*} \frac{\partial \rho^{\prime}}{\partial y^{*}}=0 \\
\frac{\partial \rho^{\prime}}{\partial t^{*}}+\bar{u}^{*} \frac{\partial \rho^{\prime}}{\partial x^{*}}+\bar{v}^{*} \frac{\partial \rho^{\prime}}{\partial y^{*}}+\bar{\rho}^{*}\left[\frac{\partial u^{\prime}}{\partial x^{*}}+\frac{\partial v^{\prime}}{\partial y^{*}}\right]=-\rho^{\prime}\left[\frac{\partial \bar{u}^{*}}{\partial x^{*}}+\frac{\partial \bar{v}^{*}}{\partial y^{*}}\right]-u^{\prime} \frac{\partial \bar{\rho}^{*}}{\partial x^{*}}-v^{\prime} \frac{\partial \bar{\rho}^{*}}{\partial y^{*}}
\end{gathered}
$$

Equation $(A-1.11)$ is the continuity equation for a non-uniform mean flow.

For a uniform mean flow,

$$
\begin{gathered}
\bar{\rho}=\bar{\rho}_{0} \quad ; \quad \bar{u}=\bar{u}_{0} \quad ; \quad \bar{v}=\bar{v}_{0} \\
\bar{\rho}^{*}=\frac{\bar{\rho}}{\bar{\rho}_{0}}=\frac{\bar{\rho}_{0}}{\bar{\rho}_{0}}=1 \Rightarrow \frac{\partial \bar{\rho}^{*}}{\partial x^{*}}=\frac{\partial \bar{\rho}^{*}}{\partial y^{*}}=0
\end{gathered}
$$




\section{APPENDIX (continued)}

Similarly,

$$
\bar{u}^{*}=\frac{\bar{u}}{a_{0}}=\frac{\bar{u}_{0}}{a_{0}}=M \cos \alpha=M_{x} \Rightarrow \frac{\partial \bar{u}^{*}}{\partial x^{*}}=0
$$

and,

$$
\bar{v}^{*}=\frac{\bar{v}}{a_{0}}=\frac{\bar{v}_{0}}{a_{0}}=M \sin \alpha=M_{y} \Rightarrow \frac{\partial \bar{v}^{*}}{\partial y^{*}}=0
$$

Then, the continuity equation for uniform mean flow is shown to be,

$$
\frac{\partial \rho^{\prime}}{\partial t^{*}}+\bar{u}^{*} \frac{\partial \rho^{\prime}}{\partial x^{*}}+\bar{v}^{*} \frac{\partial \rho^{\prime}}{\partial y^{*}}+\left[\frac{\partial u^{\prime}}{\partial x^{*}}+\frac{\partial v^{\prime}}{\partial y^{*}}\right]=0
$$

\section{Equation for the X-Momentum}

Using equations (A-1.7) and (A-1.8), the momentum equation for the $u$ component for the perturbation case is written as;

$$
\frac{\partial(\rho u-\bar{\rho} \bar{u})}{\partial t}+\frac{\partial\left[\left(\rho u^{2}+p\right)-\left(\bar{\rho}^{2}+\bar{p}\right)\right]}{\partial x}+\frac{\partial[(\rho u v)-(\overline{\rho u v})]}{\partial y}=0
$$

In non-dimensional form;

$$
\begin{aligned}
\frac{\partial(\rho u-\bar{\rho} \bar{u})}{\partial t} & =\frac{\partial\left[\rho^{*} \bar{\rho}_{0} u^{*} a_{0}-\bar{\rho}^{* *} \bar{\rho}_{0} \bar{u}^{*} a_{0}\right]}{\partial\left(t^{*} \frac{L}{a_{0}}\right)} \\
& =\frac{a_{0}^{2} \bar{\rho}_{0}}{L} \frac{\partial}{\partial t^{*}}\left[\rho^{*} u^{*}-\bar{\rho}^{* *} \bar{u}\right] \\
& =\frac{a_{0}^{2} \bar{\rho}_{0}}{L} \frac{\partial}{\partial t^{*}}\left[\left(\bar{\rho}^{*}+\rho^{\prime}\right)\left(\bar{u}^{*}+u^{\prime}\right)-\bar{\rho}^{* *} u\right.
\end{aligned}
$$




\section{APPENDIX (continued)}

$$
\begin{array}{r}
=\frac{a_{0}^{2} \bar{\rho}_{0}}{L} \frac{\partial}{\partial t^{*}}\left[\bar{\rho}^{*} \mathbb{u}^{*}+\bar{\rho}^{*} u^{\prime}+\rho^{\prime} \bar{u}^{*}+\nu^{\prime}\left\langle-\bar{\rho}^{*} u\right.\right. \\
\frac{\partial(\rho u-\bar{\rho} u)}{\partial t}=\frac{a_{0}^{2} \bar{\rho}_{0}}{L}\left[\bar{\rho}^{*} \frac{\partial u^{\prime}}{\partial t^{*}}+u^{\prime} \frac{\partial \bar{\rho}^{*}}{\partial t^{*}}+\rho^{\prime} \frac{\partial \bar{u}^{*}}{\partial t^{*}}+\bar{u}^{*} \frac{\partial \rho^{\prime}}{\partial t^{*}}\right]
\end{array}
$$

The partial derivative with respect to $x$ is;

$$
\begin{aligned}
& \frac{\partial\left[\left(\rho u^{2}+p\right)-\left(\overline{\rho u}{ }^{2}+\bar{p}\right)\right]}{\partial x}= \\
& =\frac{\partial\left[\left(\rho^{*} \bar{\rho}_{0} u^{*^{2}} a_{0}^{2}+p^{*} \bar{\rho}_{0} a_{0}^{2}\right)-\left(\bar{\rho}^{*} \bar{\rho}_{0} \bar{u}^{* 2} a_{0}^{2}+\bar{p}^{*} \bar{\rho}_{0} a_{0}^{2}\right)\right]}{\partial\left(x^{*} L\right)} \\
& =\frac{\bar{\rho}_{0} a_{0}^{2}}{L} \frac{\partial\left[\left(\rho^{*} u^{* 2}+p^{*}\right)-\left(\bar{\rho}^{*-u^{2}}+\bar{p}^{*}\right)\right]}{\partial x^{*}} \\
& =\frac{\bar{\rho}_{0} a_{0}^{2}}{L} \frac{\partial\left[\left(\left(\bar{\rho}^{*}+\rho^{\prime}\right)\left(\bar{u}^{*}+u^{\prime}\right)^{2}+\left(\bar{p}^{*}+p^{\prime}\right)\right)-\left(\bar{\rho}^{*} \bar{u}^{* 2}+\bar{p}^{*}\right)\right]}{\partial x^{*}} \\
& =\frac{\bar{\rho}_{0} a_{0}^{2}}{L} \frac{\partial\left[\left(\left(\bar{\rho}^{*}+\rho^{\prime}\right)\left(\bar{u}^{* 2}+2 \bar{u}^{*} u^{\prime}+u^{\prime 2}\right)+\left(\bar{p}^{*}+p^{\prime}\right)\right)-\left(\bar{\rho}^{*} \bar{u}^{-*^{2}}+\bar{p}^{*}\right)\right]}{\partial x^{*}}
\end{aligned}
$$

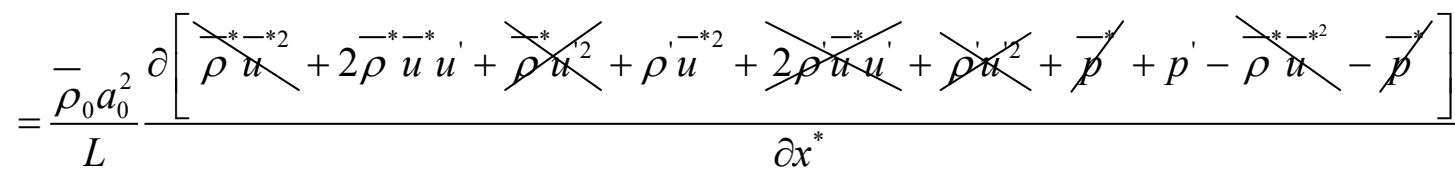

$$
\begin{aligned}
& =\frac{\bar{\rho}_{0} a_{0}^{2}}{L}\left[2 \bar{\rho}^{*} \frac{\partial\left(\bar{u}^{*} u^{\prime}\right)}{\partial x^{*}}+2 \bar{u}^{*} u^{\prime} \frac{\partial \bar{\rho}^{*}}{\partial x^{*}}+2 \rho^{\prime-u^{*}} \frac{\partial \bar{u}^{*}}{\partial x^{*}}+\bar{u}^{* 2} \frac{\partial \rho^{\prime}}{\partial x^{*}}+\frac{\partial p^{\prime}}{\partial x^{*}}\right]
\end{aligned}
$$




\section{APPENDIX (continued)}

$$
\frac{\partial\left[\left(\rho u^{2}+p\right)-\left(\bar{\rho} \bar{u}^{2}+\bar{p}\right)\right]}{\partial x}=\frac{\bar{\rho}_{0} a_{0}^{2}}{L}\left[\begin{array}{l}
2 \bar{\rho}^{*} \bar{u}^{*} \frac{\partial u^{\prime}}{\partial x^{*}}+2 \bar{\rho}^{*} u^{\prime} \frac{\partial \bar{u}^{*}}{\partial x^{*}}+2 \bar{u}^{*} u^{\prime} \frac{\partial \bar{\rho}^{*}}{\partial x^{*}}+ \\
2 \rho^{\prime-\bar{u}^{*}} \frac{\partial \bar{u}^{*}}{\partial x^{*}}+\bar{u}^{*} \frac{\partial \rho^{\prime}}{\partial x^{*}}+\frac{\partial p^{\prime}}{\partial x^{*}}
\end{array}\right]
$$

and the partial derivative with respect to $y$ is;

$$
\begin{aligned}
& \frac{\partial[(\rho u v)-(\overline{\rho u v})]}{\partial y}= \\
& =\frac{\partial\left[\left(\rho^{*} \bar{\rho}_{0} u^{*} a_{0} v^{*} a_{0}\right)-\left(\bar{\rho}^{*} \bar{\rho}_{0} \bar{u}^{*} a_{0} \bar{v}^{*} a_{0}\right)\right]}{\partial\left(y^{*} L\right)} \\
& =\frac{\bar{\rho}_{0} a_{0}^{2}}{L} \frac{\partial\left[\left(\rho^{*} u^{*} v^{*}\right)-\left(\bar{\rho}^{*} \bar{u}^{* *} v\right)\right]}{\partial y^{*}} \\
& =\frac{\bar{\rho}_{0} a_{0}^{2}}{L} \frac{\partial\left[\left(\left(\bar{\rho}^{*}+\rho^{\prime}\right)\left(\bar{u}^{*}+u^{\prime}\right)\left(\bar{v}^{*}+v^{\prime}\right)\right)-\left(\bar{\rho}^{*} \bar{u}^{*} \bar{v}^{*}\right)\right]}{\partial y^{*}} \\
& =\frac{\bar{\rho}_{0} a_{0}^{2}}{L} \frac{\partial\left[\left(\left(\bar{\rho}^{*} \bar{u}^{*}+\bar{\rho}^{*} u^{\prime}+\rho^{\prime \bar{u}^{*}}+\rho^{\prime} u^{\prime}\right)\left(\bar{v}^{*}+v^{\prime}\right)\right)-\left(\bar{\rho}^{*-*} u^{*} v^{*}\right)\right]}{\partial y^{*}}
\end{aligned}
$$

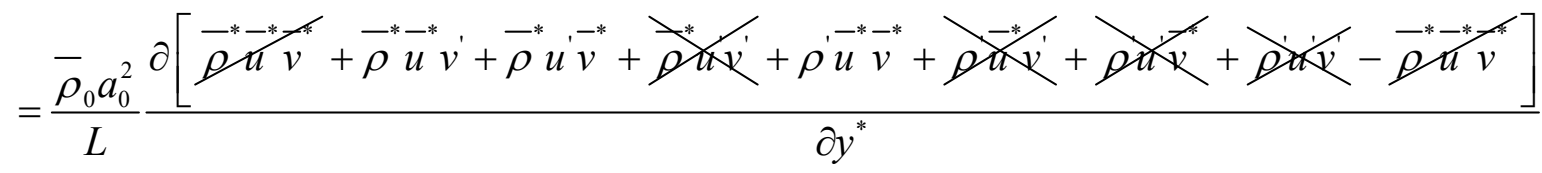

$$
\begin{aligned}
& =\frac{\bar{\rho}_{0} a_{0}^{2}}{L}\left[\bar{\rho}^{*} \frac{\partial\left(\bar{u}^{*} v^{\prime}\right)}{\partial y^{*}}+\bar{u}^{*} v^{\prime} \frac{\partial \bar{\rho}^{*}}{\partial y^{*}}+\bar{\rho}^{*} \frac{\partial\left(u^{-v^{*}}\right)}{\partial y^{*}}+u^{-*} \bar{v}^{*} \frac{\partial \bar{\rho}^{*}}{\partial y^{*}}+\rho^{\prime} \frac{\partial\left(\bar{u}^{*}-^{*}\right)}{\partial y^{*}}+\bar{u}^{*-*} v \frac{\partial \rho^{\prime}}{\partial y^{*}}\right]
\end{aligned}
$$




\section{APPENDIX (continued)}

$$
\frac{\partial[(\rho u v)-(\overline{\rho u v})]}{\partial y}=\frac{\bar{\rho}_{0} a_{0}^{2}}{L}\left[\begin{array}{l}
\bar{\rho}^{*} \bar{u}^{*} \frac{\partial v^{\prime}}{\partial y^{*}}+\bar{\rho}^{*} v^{\prime} \frac{\partial \bar{u}^{*}}{\partial y^{*}}+\bar{u}^{*} v^{\prime} \frac{\partial \bar{\rho}^{*}}{\partial y^{*}}+\bar{\rho}^{*} u^{\prime} \frac{\partial \vec{v}^{*}}{\partial y^{*}}+\bar{\rho}^{*} v^{*} \frac{\partial u^{\prime}}{\partial y^{*}}+ \\
u^{\prime} v^{*} \frac{\partial \bar{\rho}^{*}}{\partial y^{*}}+\rho^{\prime} \bar{u}^{*} \frac{\partial \bar{v}^{*}}{\partial y^{*}}+\rho^{\prime-*} \frac{\partial \bar{u}^{*}}{\partial y^{*}}+\bar{u}^{* *-v^{*}} \frac{\partial \rho^{\prime}}{\partial y^{*}}
\end{array}\right]
$$

Substituting equations (A-1.17) to (A-1.19) in equation (A-1.16),

$$
\begin{aligned}
& \frac{a_{0}^{2} \bar{\rho}_{0}}{L}\left[\bar{\rho}^{*} \frac{\partial u^{\prime}}{\partial t^{*}}+u^{\prime} \frac{\partial \bar{\rho}^{*}}{\partial t^{*}}+\rho^{\prime} \frac{\partial \bar{u}^{*}}{\partial t^{*}}+\bar{u}^{*} \frac{\partial \rho^{\prime}}{\partial t^{*}}\right]+ \\
& \frac{\bar{\rho}_{0} a_{0}^{2}}{L}\left[2 \bar{\rho}^{*} \bar{u}^{*} \frac{\partial u^{\prime}}{\partial x^{*}}+2 \bar{\rho}^{*} u^{\prime} \frac{\partial \bar{u}^{*}}{\partial x^{*}}+2 \bar{u}^{*} u^{\prime} \frac{\partial \bar{\rho}^{*}}{\partial x^{*}}+2 \rho^{\prime} \bar{u}^{*} \frac{\partial \bar{u}^{*}}{\partial x^{*}}+\bar{u}^{*} \frac{\partial \rho^{\prime}}{\partial x^{*}}+\frac{\partial p^{\prime}}{\partial x^{*}}\right]+ \\
& \frac{\bar{\rho}_{0} a_{0}^{2}}{L}\left[\begin{array}{l}
\bar{\rho}^{*} \bar{u}^{*} \frac{\partial v^{\prime}}{\partial y^{*}}+\bar{\rho}^{*} v^{\prime} \frac{\partial \bar{u}^{*}}{\partial y^{*}}+\bar{u}^{*} v^{\prime} \frac{\partial \bar{\rho}^{*}}{\partial y^{*}}+\bar{\rho}^{*} u^{\prime} \frac{\partial \bar{v}^{*}}{\partial y^{*}}+ \\
\bar{\rho}^{*-*} v \frac{\partial u^{\prime}}{\partial y^{*}}+u^{\prime * *} v \frac{\partial \bar{\rho}^{*}}{\partial y^{*}}+\rho^{\prime-*} \frac{\partial \bar{v}^{*}}{\partial y^{*}}+\rho^{\prime-v^{*}} \frac{\partial \bar{u}^{*}}{\partial y^{*}}+\bar{u}^{*}-^{*} \frac{\partial \rho^{\prime}}{\partial y^{*}}
\end{array}\right]=0 \\
& \frac{\partial \bar{\rho}^{*}}{\partial t^{*}}=\frac{\partial \bar{u}^{*}}{\partial t^{*}}=0 \text { for steady mean flow. }
\end{aligned}
$$

Cancelling $\frac{a_{0}^{2} \bar{\rho}_{0}}{L}$ throughout and substituting for $\frac{\partial \rho^{\prime}}{\partial t^{*}}$ from the continuity equation given by equation (A-1.11), equation (A-120) becomes, 


\section{APPENDIX (continued)}

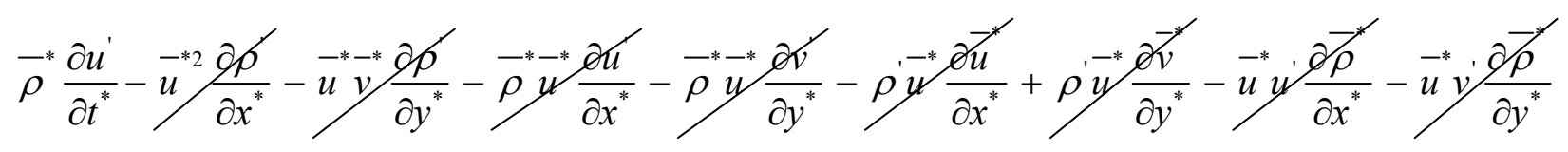

$$
\begin{aligned}
& \not 2 \bar{\rho}^{*-*} u^{*} \frac{\partial u^{\prime}}{\partial x^{*}}+2 \bar{\rho}^{*} u^{\prime} \frac{\partial \bar{u}^{*}}{\partial x^{*}}+\not 2 u^{* *} u^{\prime} \frac{\partial \bar{\rho}^{*}}{\partial x^{*}}+\not 2 \rho^{\prime} u^{* *} \frac{\partial u^{*}}{\partial x^{*}}+\bar{u}^{* 2} \frac{\partial \rho}{\partial x^{*}}+\frac{\partial p^{\prime}}{\partial x^{*}}+
\end{aligned}
$$

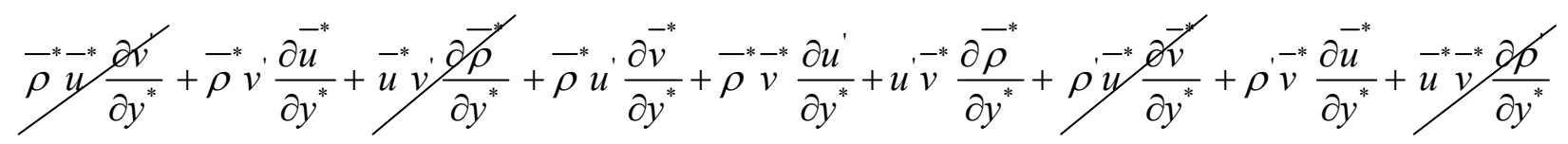

$$
\begin{aligned}
& = \\
& 0
\end{aligned}
$$

Dividing the equation by $\bar{\rho}^{*}$,

$$
\begin{aligned}
& \frac{\partial u^{\prime}}{\partial t^{*}}+\bar{u}^{*} \frac{\partial u^{\prime}}{\partial x^{*}}+2 u^{\prime} \frac{\partial \bar{u}^{*}}{\partial x^{*}}+\frac{\bar{u}^{*}}{\bar{\rho}^{*}} u^{\prime} \frac{\partial \bar{\rho}^{*}}{\partial x^{*}}+\frac{\rho^{\prime}}{\bar{\rho}^{*}} \bar{u}^{*} \frac{\partial \bar{u}^{*}}{\partial x^{*}}+\frac{\partial p}{\partial x^{*}}+v^{\prime} \frac{\partial \bar{u}^{*}}{\partial y^{*}}+u^{\prime} \frac{\partial \bar{v}^{*}}{\partial y^{*}}+ \\
& \bar{v}^{*} \frac{\partial u}{\partial y^{*}}+\frac{u^{\prime}}{\bar{\rho}^{*}} \bar{v}^{*} \frac{\partial \bar{\rho}^{*}}{\partial y^{*}}+\frac{\rho^{\prime}}{\bar{\rho}^{*}} \bar{v}^{*} \frac{\partial \bar{u}^{*}}{\partial y^{*}}=0
\end{aligned}
$$

Rearranging the terms,

$$
\begin{gathered}
\frac{\partial u^{\prime}}{\partial t^{*}}+\bar{u}^{*} \frac{\partial u^{\prime}}{\partial x^{*}}+\bar{v}^{*} \frac{\partial u^{\prime}}{\partial y^{*}}++\frac{1}{\bar{\rho}^{*}} \frac{\partial p^{\prime}}{\partial x^{*}} \\
=-u^{\prime} \frac{\partial \bar{u}^{*}}{\partial x^{*}}-v^{\prime} \frac{\partial \bar{u}^{*}}{\partial y^{*}}-u^{\prime}\left[\frac{\partial \bar{u}^{*}}{\partial x^{*}}+\frac{\partial \bar{v}^{*}}{\partial y^{*}}\right]-\frac{u^{\prime}}{\bar{\rho}^{*}}\left[\bar{u}^{*} \frac{\partial \bar{\rho}^{*}}{\partial x^{*}}+\bar{v}^{*} \frac{\partial \bar{\rho}^{*}}{\partial y^{*}}\right]-\frac{\rho^{\prime}}{\bar{\rho}^{*}}\left[\bar{u}^{*} \frac{\partial \bar{u}^{*}}{\partial x^{*}}+\bar{v}^{*} \frac{\partial \bar{u}^{*}}{\partial y^{*}}\right](\mathrm{A}-1
\end{gathered}
$$




\section{APPENDIX (continued)}

Equation $(\mathrm{A}-1.21)$ is the equation for $\mathrm{X}$-momentum in perturbation quantities. This equation can be further simplified through the non-uniform mean flow pressure term. It is derived by the following approach.

From the steady mean flow equations, the $\mathrm{X}$-momentum equation is;

$$
\frac{\partial(\overline{\rho u}+\bar{p})}{\partial x}+\frac{\partial(\bar{\rho} \overline{u v})}{\partial y}=0
$$

In non-dimensional form;

$$
\begin{aligned}
& \frac{\partial\left(\bar{\rho}^{*} \bar{\rho}_{0} \bar{u}^{* 2} a_{0}^{2}+\bar{p}^{*} \overline{\rho_{0}} a_{0}^{2}\right)}{\partial\left(x^{*} L\right)}+\frac{\partial\left(\bar{\rho}^{*} \bar{\rho}_{0} \bar{u}^{*} a_{0} \bar{v}^{*} a_{0}\right)}{\partial\left(y^{*} L\right)}=0 \\
& \frac{\bar{\rho}_{0} a_{0}^{2}}{L}\left[\frac{\partial\left(\bar{\rho}^{*} u^{* 2}+\bar{p}^{*}\right)}{\partial x^{*}}+\frac{\partial\left(\bar{\rho}^{*} \bar{u}^{*}-^{*}\right)}{\partial y^{*}}\right]=0 \\
& \frac{\bar{\rho}_{0} a_{0}^{2}}{L}\left[2 \bar{\rho}^{*} \bar{u} \frac{\partial \bar{u}^{*}}{\partial x^{*}}+\bar{u}^{* 2} \frac{\partial \bar{\rho}^{*}}{\partial x^{*}}+\frac{\partial \bar{p}^{*}}{\partial x^{*}}+\bar{\rho}^{*} \frac{\partial\left(\bar{u}^{*}-^{*}\right)}{\partial y^{*}}+\bar{u}^{*}-^{*} \frac{\partial \bar{\rho}^{*}}{\partial y^{*}}\right]=0 \\
& 2 \bar{\rho}^{*} \bar{u}^{*} \frac{\partial \vec{u}^{*}}{\partial x^{*}}+\bar{u}^{*} 2 \frac{\partial \bar{\rho}^{*}}{\partial x^{*}}+\frac{\partial \bar{p}^{*}}{\partial x^{*}}+\bar{\rho}^{*} \vec{u} \frac{\partial \vec{v}^{*}}{\partial y^{*}}+\bar{\rho}^{*-*} v \frac{\partial \bar{u}^{*}}{\partial y^{*}}+\bar{u}^{*} v-{ }^{*} \frac{\partial \bar{\rho}^{*}}{\partial y^{*}}=0 \\
& \frac{\partial \bar{p}^{*}}{\partial x^{*}}=-2 \bar{\rho}^{*} \bar{u}^{*} \frac{\partial \bar{u}^{*}}{\partial x^{*}}-\bar{u}^{* 2} \frac{\partial \bar{\rho}^{*}}{\partial x^{*}}-\bar{\rho}^{*} \bar{u}^{*} \frac{\partial \bar{v}^{*}}{\partial y^{*}}-\bar{\rho}^{*}{ }^{*} v \frac{\partial \bar{u}^{*}}{\partial y^{*}}-\bar{u}^{*-*} v \frac{\partial \bar{\rho}^{*}}{\partial y^{*}} \\
& \frac{\partial \vec{p}^{*}}{\partial x^{*}} \frac{\rho^{\prime}}{\bar{\rho}^{* 2}}=-2 \frac{\rho^{\prime}}{\bar{\rho}^{*}} \bar{u}^{*} \frac{\partial \vec{u}^{*}}{\partial x^{*}}-\bar{u}^{* 2} \frac{\rho^{\prime}}{\bar{\rho}^{*}} \frac{\partial \bar{\rho}^{*}}{\partial x^{*}}-\bar{u}^{*} \frac{\rho^{\prime}}{\bar{\rho}^{*}} \frac{\partial \vec{v}^{*}}{\partial y^{*}}-\frac{\rho^{\prime}}{\bar{\rho}^{*}} \bar{v}^{*} \frac{\partial \vec{u}^{*}}{\partial y^{*}}-\bar{u}^{*}-^{*} \frac{\rho}{\bar{\rho}^{*}} \frac{\partial \bar{\rho}^{*}}{\partial y^{*}}
\end{aligned}
$$




\section{APPENDIX (continued)}

$$
\frac{\partial \bar{p}^{*}}{\partial x^{*}} \frac{\rho^{\prime}}{\bar{\rho}^{* 2}}=-\frac{\rho^{\prime}}{\bar{\rho}^{*}}\left[\bar{u}^{*} \frac{\partial \bar{u}^{*}}{\partial x^{*}}+\bar{v}^{*} \frac{\partial \bar{u}^{*}}{\partial y^{*}}\right]-\bar{u}^{*} \frac{\rho^{\prime}}{\bar{\rho}^{*}}\left[\frac{\partial \bar{u}^{*}}{\partial y^{*}}+\frac{\partial \bar{v}^{*}}{\partial y^{*}}\right]-\bar{u}^{*} \frac{\rho^{\prime}}{\bar{\rho}^{* 2}}\left[\frac{\partial \bar{\rho}^{*}}{\partial x^{*}}+\bar{v}^{*} \frac{\partial \bar{\rho}^{*}}{\partial y^{*}}\right]
$$

Now, consider the continuity equation for the steady mean flow.

$$
\frac{\partial(\bar{\rho} \bar{u})}{\partial x}+\frac{\partial(\bar{\rho} \bar{v})}{\partial y}=0
$$

which in non-dimensional form is,

$$
\begin{gathered}
\frac{\partial\left(\bar{\rho}^{*} \bar{\rho}_{0} \bar{u}^{*} a_{0}\right)}{\partial\left(x^{*} L\right)}+\frac{\partial\left(\bar{\rho}^{*} \bar{\rho}_{0} v a_{0}\right)}{\partial\left(y^{*} L\right)}=0 \\
\frac{\bar{\rho}_{0} a_{0}}{L}\left[\frac{\partial\left(\bar{\rho}^{*} \bar{u}^{*}\right)}{\partial x^{*}}+\frac{\partial\left(\bar{\rho}^{*} \bar{v}^{*}\right)}{\partial y^{*}}\right]=0 \\
\bar{\rho}^{*} \frac{\partial \bar{u}^{*}}{\partial x^{*}}+\bar{u}^{*} \frac{\partial \bar{\rho}^{*}}{\partial x^{*}}+\bar{\rho}^{*} \frac{\partial \bar{v}^{*}}{\partial y^{*}}+\bar{v}^{*} \frac{\partial \bar{\rho}^{*}}{\partial y^{*}}=0 \\
\bar{\rho}^{*}\left[\frac{\partial \bar{u}^{*}}{\partial x^{*}}+\frac{\partial \bar{v}^{*}}{\partial y^{*}}\right]+\left[\bar{u}^{*} \frac{\partial \bar{\rho}^{*}}{\partial x^{*}}+\bar{v}^{*} \frac{\partial \bar{\rho}^{*}}{\partial y^{*}}\right]=0 \\
-\frac{1}{\bar{\rho}^{*}}\left[\bar{u}^{*} \frac{\partial \bar{\rho}^{*}}{\partial x^{*}}+\bar{v}^{*} \frac{\partial \bar{\rho}^{*}}{\partial y^{*}}\right]=\left[\frac{\partial \vec{u}^{*}}{\partial x^{*}}+\frac{\partial \vec{v}^{*}}{\partial y^{*}}\right]
\end{gathered}
$$

Substituting equation (A-1.23) in equation (A-1.22), 


\section{APPENDIX (continued)}

$$
\begin{aligned}
& \frac{\partial \bar{p}^{*}}{\partial x^{*}} \frac{\rho^{\prime}}{\bar{\rho}^{* 2}}=-\frac{\rho^{\prime}}{\bar{\rho}^{*}}\left[\vec{u}^{*} \frac{\partial \bar{u}^{*}}{\partial x^{*}}+\bar{v}^{*} \frac{\partial \vec{u}^{*}}{\partial y^{*}}\right]+\vec{u}^{*} \frac{\rho^{\prime}}{\bar{\rho}^{* 2}}\left[\frac{\left.\bar{u}^{*} \frac{\partial \bar{\rho}^{*}}{\partial x^{*}+v} \frac{\partial \rho}{\partial y^{*}}\right]}{\bar{u}^{*} \frac{\rho^{\prime}}{\bar{\rho}^{*}}\left[\frac{\partial \bar{\rho}^{*}}{\partial x^{*}+v} \frac{\partial \rho}{\partial y^{*}}\right]}\right.
\end{aligned}
$$

Substitution of equation (A-1.23) in equation (A-1.21) provides,

$$
\begin{aligned}
& \frac{\partial u^{\prime}}{\partial t^{*}}+\bar{u}^{*} \frac{\partial u^{\prime}}{\partial x^{*}}+\bar{v}^{*} \frac{\partial u^{\prime}}{\partial y^{*}}++\frac{1}{\bar{\rho}^{*}} \frac{\partial p^{\prime}}{\partial x^{*}} \\
& =-u^{\prime} \frac{\partial \vec{u}^{*}}{\partial x^{*}}-v^{\prime} \frac{\partial \vec{u}^{*}}{\partial y^{*}}+\frac{u^{\prime}}{\bar{\rho}^{*}}\left[\bar{u}^{*} \frac{\partial \vec{\rho}^{*}}{\partial x^{*}}+\vec{v} \frac{\partial \rho}{\partial y^{*}}\right] \\
& -\frac{u^{\prime}}{\bar{\rho}^{*}}\left[\vec{u}^{*} \frac{\partial \bar{\rho}^{*}}{\partial x^{*}+v} \frac{\partial \bar{\rho}}{\partial y^{*}}\right]-\frac{\rho^{\prime}}{\bar{\rho}^{*}}\left[\vec{u}^{*} \frac{\partial \bar{u}^{*}}{\partial x^{*}}+\bar{v}^{*} \frac{\partial \bar{u}^{*}}{\partial y^{*}}\right]
\end{aligned}
$$

Utilizing equation ( $\mathrm{A}-1.24)$ in eqaution ( $\mathrm{A}-1.25)$, the equation for $\mathrm{X}$-momentum of steady non-uniform mean flow is obtained:

$$
\frac{\partial u^{\prime}}{\partial t^{*}}+\bar{u}^{*} \frac{\partial u^{\prime}}{\partial x^{*}}+\bar{v}^{*} \frac{\partial u^{\prime}}{\partial y^{*}}+\frac{1}{\bar{\rho}^{*}} \frac{\partial p^{\prime}}{\partial x^{*}}=-u^{\prime} \frac{\partial \bar{u}^{*}}{\partial x^{*}}-v^{\prime} \frac{\partial \bar{u}^{*}}{\partial y^{*}}+\frac{\partial \bar{p}^{*}}{\partial x^{*}} \frac{\rho^{\prime}}{\bar{\rho}^{* 2}}
$$

Equation $(\mathrm{A}-1.26)$ is the $\mathrm{X}$-momentum equation for the non-uniform mean flow in perturbation terms.

From equations (A-1.12) to (A-1.15), the equation for $X$-momentum for uniform flow in terms of perturbation quantities is given as,

$$
\frac{\partial u^{\prime}}{\partial t^{*}}+M_{x} \frac{\partial u^{\prime}}{\partial x^{*}}+M_{y} \frac{\partial u^{\prime}}{\partial y^{*}}+\frac{\partial p^{\prime}}{\partial x^{*}}=0
$$




\section{APPENDIX (continued)}

Following the similar steps, Y-momentum equation of a non-uniform mean flow in terms of perturbation quantities can be obtained and expressed as,

$$
\frac{\partial v^{\prime}}{\partial t^{*}}+\bar{u}^{*} \frac{\partial v^{\prime}}{\partial x^{*}}+\bar{v}^{*} \frac{\partial v^{\prime}}{\partial y^{*}}+\frac{1}{\bar{\rho}^{*}} \frac{\partial p^{\prime}}{\partial y^{*}}=-u^{\prime} \frac{\partial \bar{v}^{*}}{\partial x^{*}}-v^{\prime} \frac{\partial \bar{v}^{*}}{\partial y^{*}}+\frac{\partial \bar{p}^{*}}{\partial y^{*}} \frac{\rho^{\prime}}{\bar{\rho}^{* 2}}
$$

Similarly the uniform mean flow is expressed as,

$$
\frac{\partial v^{\prime}}{\partial t^{*}}+M_{x} \frac{\partial v^{\prime}}{\partial x^{*}}+M_{y} \frac{\partial v^{\prime}}{\partial y^{*}}+\frac{\partial p^{\prime}}{\partial y^{*}}=0
$$

\section{Equation for Energy}

Using equations (A-1.6) and (A-1.7), the Energy equation for the perturbation variables is derived as follows;

$$
\begin{aligned}
& \frac{\partial(\rho e-\bar{\rho} \bar{e})}{\partial t}+ \\
& \frac{\partial[((\rho e+p) u)-((\bar{\rho} \bar{e}+\bar{p}) \bar{u})]}{\partial x}+\frac{\partial[((\rho e+p) v)-((\overline{\rho e}+\bar{p}) \bar{v})]}{\partial y}=0
\end{aligned}
$$




\section{APPENDIX (continued)}

In non-dimensional form;

$$
\begin{aligned}
& \frac{\partial(\rho e-\bar{\rho} e)}{\partial t}=\frac{\partial\left[\rho^{*} \bar{\rho}_{0}\left(\frac{p^{*} \bar{p}_{0} a_{0}^{2}}{\rho^{*} \bar{\not}_{0}(\gamma-1)}+\frac{1}{2}\left(u^{* 2} a_{0}^{2}+v^{* 2} a_{0}^{2}\right)\right)-\bar{\rho}^{*} \bar{\rho}_{0}\left(\frac{\bar{p}^{*} \bar{p}_{0} a_{0}^{2}}{\bar{\rho}^{*} \bar{\not}_{0}(\gamma-1)}+\frac{1}{2}\left(\bar{u}^{* 2} a_{0}^{2}+\bar{v}^{* 2} a_{0}^{2}\right)\right)\right]}{\partial\left(t^{*} \frac{L}{a_{0}}\right)}
\end{aligned}
$$

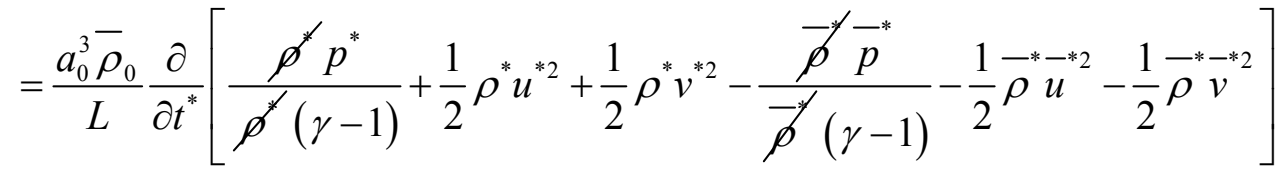

$$
\begin{aligned}
& =\frac{a_{0}^{3} \bar{\rho}_{0}}{L} \frac{\partial}{\partial t^{*}}\left[\begin{array}{l}
\frac{\left(\bar{p}^{*}+p^{\prime}\right)}{(\gamma-1)}+\frac{1}{2}\left(\bar{\rho}^{*}+\rho^{\prime}\right)\left(\bar{u}^{*}+u^{\prime}\right)^{2}+\frac{1}{2}\left(\bar{\rho}^{*}+\rho^{\prime}\right)\left(\bar{v}^{*}+v^{\prime}\right)^{2}- \\
\frac{\bar{p}^{*}}{(\gamma-1)}-\frac{1}{2} \bar{\rho}^{*}-^{* 2}-\frac{1}{2} \bar{\rho}^{*-*^{*}}
\end{array}\right]
\end{aligned}
$$

Expanding equation (A-1.31) and neglecting the product of perturbation quantities for their small values, equation (A-1.31) becomes,

$$
\begin{aligned}
& =\frac{a_{0}^{3} \bar{\rho}_{0}}{L} \frac{\partial}{\partial t^{*}}\left[\frac{p^{\prime}}{\gamma-1}+\bar{\rho}^{*} \bar{u}^{*} u^{\prime}+\frac{1}{2} \rho^{\prime} \bar{u}^{*}+\bar{\rho}^{*} \bar{v}^{*} v^{\prime}+\frac{1}{2} \rho^{\prime-v^{*}}\right] \\
& \frac{\partial(\rho e-\bar{\rho} \bar{e})}{\partial t}=\frac{a_{0}^{3} \bar{\rho}_{0}}{L}\left[\frac{1}{\gamma-1} \frac{\partial p^{\prime}}{\partial t^{*}}+\bar{\rho}^{*} \bar{u}^{*} \frac{\partial u^{\prime}}{\partial t^{*}}+\frac{1}{2} \bar{u}^{*} \frac{\partial \rho^{\prime}}{\partial t^{*}}+\bar{\rho}^{*} \bar{v}^{*} \frac{\partial v^{\prime}}{\partial t^{*}}+\frac{1}{2} \bar{v}^{*} \frac{\partial \rho^{\prime}}{\partial t^{*}}\right]
\end{aligned}
$$




\section{APPENDIX (continued)}

Following similar steps, the $\frac{\partial}{\partial x}$ and $\frac{\partial}{\partial y}$ terms are derived as;

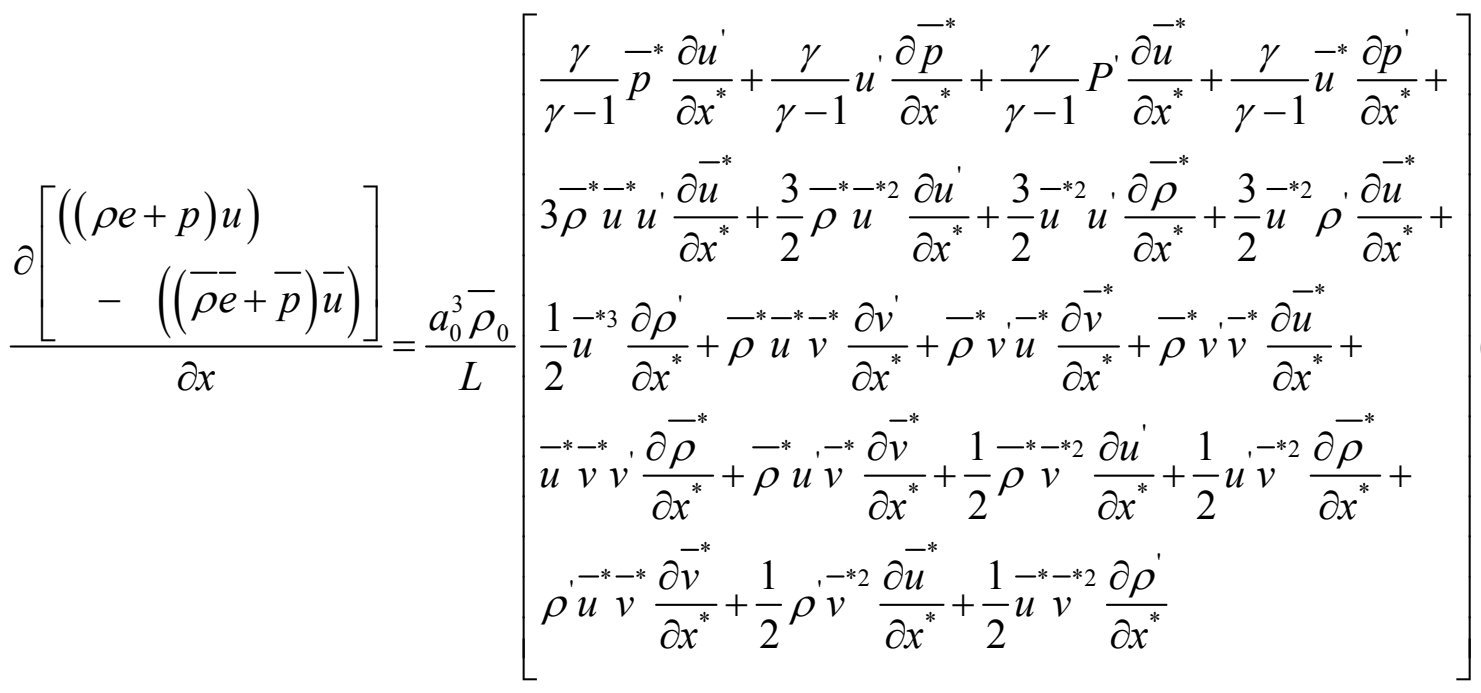

and

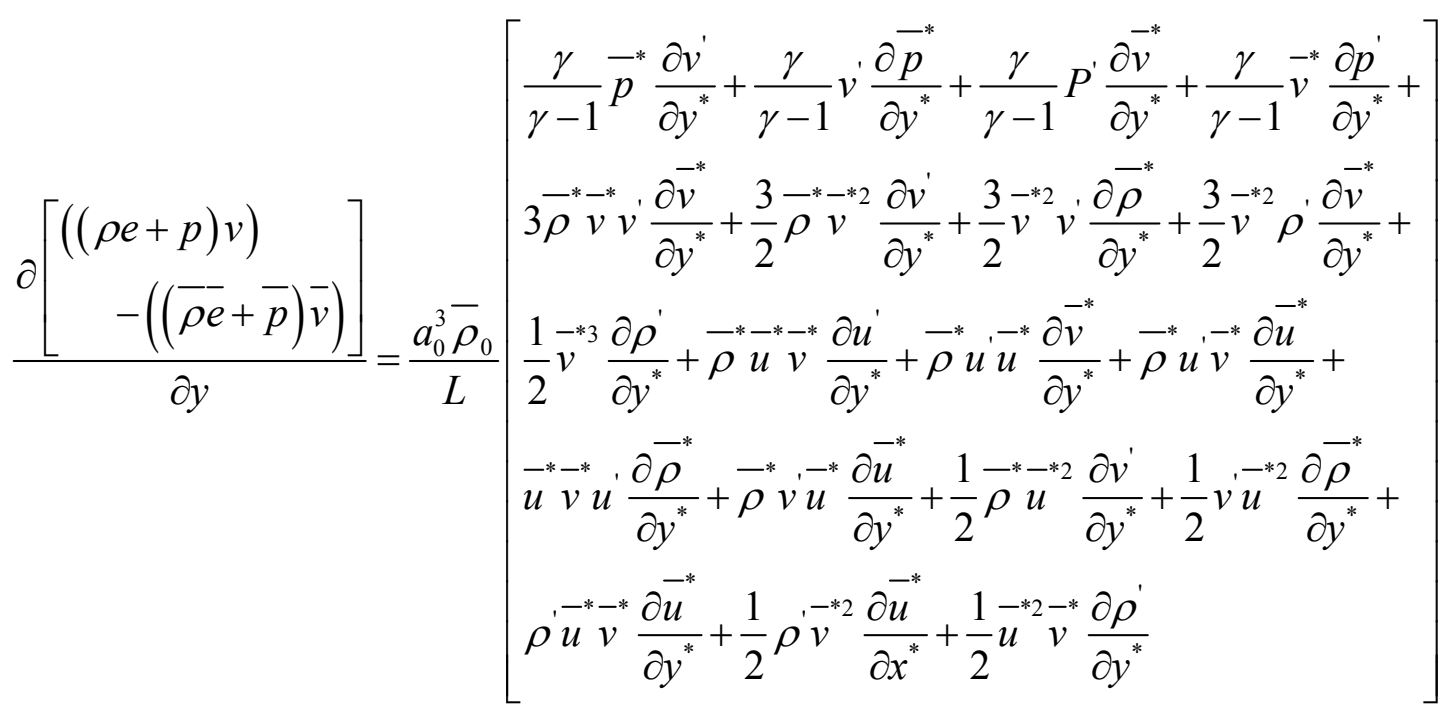




\section{APPENDIX (continued)}

Substitution of equations (A-1.32) to (A-1.34) in Equation (A-1.30) provides,

$$
\begin{aligned}
& \frac{1}{\gamma-1} \frac{\partial p^{\prime}}{\partial t^{*}}-\bar{u}^{*} \frac{\partial p^{\prime}}{\partial x^{*}}-\bar{v}^{*} \frac{\partial p^{\prime}}{\partial y^{*}}+\frac{\gamma}{\gamma-1} \bar{u}^{*} \frac{\partial p^{\prime}}{\partial x^{*}}+\frac{\gamma}{\gamma-1} \bar{v}^{*} \frac{\partial p^{\prime}}{\partial y^{*}}+\frac{\gamma}{\gamma-1} \bar{p}^{*} \frac{\partial u^{\prime}}{\partial x^{*}}+ \\
& \frac{\gamma}{\gamma-1} \bar{p}^{*} \frac{\partial v^{\prime}}{\partial y^{*}}+\frac{\gamma}{\gamma-1} u^{\prime} \frac{\partial \bar{p}^{*}}{\partial x^{*}}+\frac{\gamma}{\gamma-1} v^{\prime} \frac{\partial \bar{p}^{*}}{\partial y^{*}}+\bar{\rho}^{*} \bar{u}^{*} u^{\prime} \frac{\partial \bar{u}^{*}}{\partial x^{*}}+\bar{\rho}^{*}-^{*} u^{\prime} \frac{\partial \vec{u}^{*}}{\partial y^{*}}+ \\
& \bar{\rho}^{*} \bar{u}^{*} v^{\prime} \frac{\partial \bar{v}^{*}}{\partial x^{*}}+\bar{\rho}^{*} \bar{v}^{*} v^{\prime} \frac{\partial \bar{v}^{*}}{\partial y^{*}}+\bar{\rho}^{*} \bar{v}^{*} u^{\prime} \frac{\partial \bar{u}^{*}}{\partial x^{*}}+\bar{\rho}^{*} \bar{v}^{*} u^{\prime} \frac{\partial \vec{u}^{*}}{\partial y^{*}}+\bar{u}^{*}-^{*} v v^{\prime} \frac{\partial \bar{\rho}^{*}}{\partial x^{*}}+ \\
& \bar{v}^{*} \bar{v}^{*} v^{\prime} \frac{\partial \bar{\rho}^{*}}{\partial y^{*}}=0
\end{aligned}
$$

Using equation (A-1.24), it can be written that,

$$
\begin{aligned}
& u^{\prime} \bar{\rho}^{*}\left[\vec{u}^{*} \frac{\partial \vec{u}^{*}}{\partial x^{*}}+\vec{v}^{*} \frac{\partial \vec{u}^{*}}{\partial y^{*}}\right]=u^{\prime} \frac{\partial \vec{p}^{*}}{\partial x^{*}} \\
& v^{\prime} \bar{\rho}^{*}\left[\vec{u}^{*} \frac{\partial \vec{v}^{*}}{\partial x^{*}}+\vec{v}^{*} \frac{\partial \vec{v}^{*}}{\partial y^{*}}\right]=v^{\prime} \frac{\partial \vec{p}^{*}}{\partial y^{*}}
\end{aligned}
$$

and using equation (A-1.23),

$$
\bar{\rho}^{*} \bar{v}^{*} v^{\prime} \frac{\partial \bar{u}^{*}}{\partial x^{*}}+\overline{\rho^{*} v^{*}} v \frac{\partial \vec{v}^{*}}{\partial y^{*}}=\bar{\rho}^{*} \bar{v}^{*} v^{\prime}\left[\frac{\partial \vec{u}^{*}}{\partial x^{*}}+\frac{\partial \vec{v}^{*}}{\partial y^{*}}\right]=\bar{\rho}^{*} \bar{v}^{*} v^{\prime}\left[\frac{1}{\bar{\rho}^{*}}\left(\vec{u}^{*} \frac{\partial \bar{\rho}^{*}}{\partial x^{*}}+\bar{v}^{*} \frac{\partial \bar{\rho}^{*}}{\partial y^{*}}\right)\right]
$$

Substitution of equations (A-1.36) and (A-1.37) in Equation (A-1.35) results in,

$$
\frac{\partial p^{\prime}}{\partial t^{*}}+\bar{u}^{*} \frac{\partial p^{\prime}}{\partial x^{*}}+\bar{v}^{*} \frac{\partial p^{\prime}}{\partial y^{*}}+\gamma \bar{p}^{*}\left(\frac{\partial u^{\prime}}{\partial x^{*}}+\frac{\partial v^{\prime}}{\partial y^{*}}\right)=-u^{\prime} \frac{\partial \bar{p}^{*}}{\partial x^{*}}-v^{\prime} \frac{\partial \bar{p}^{*}}{\partial y^{*}}-\gamma\left(\frac{\partial \bar{u}^{*}}{\partial x^{*}}+\frac{\partial \vec{v}^{*}}{\partial y^{*}}\right) p^{\prime}
$$

Equation (A-1.38) is the Energy equation for non-uniform mean flow in terms of perturbation variables.

As discussed previously, for a uniform mean flow, 


$$
\begin{gathered}
\text { APPENDIX (continued) } \\
\bar{p}=\bar{p}_{0} \quad ; \quad \bar{u}=\bar{u}_{0} \quad ; \quad \bar{v}=\bar{v}_{0} \\
\bar{p}^{*}=\frac{\bar{p}}{\bar{\rho}_{0} a_{0}^{2}}=\frac{\bar{p}_{0}}{\gamma \bar{p}_{0}}=\frac{1}{\gamma} \Rightarrow \frac{\partial \bar{p}^{*}}{\partial x^{*}}=\frac{\partial \bar{p}^{*}}{\partial y^{*}}=0
\end{gathered}
$$

Using Equations (A-1.12) - (A-1.15), the equation of energy for uniform flow is given by,

$$
\frac{\partial p^{\prime}}{\partial t^{*}}+M_{x} \frac{\partial p^{\prime}}{\partial x^{*}}+M_{y} \frac{\partial p^{\prime}}{\partial y^{*}}+\left(\frac{\partial u^{\prime}}{\partial x^{*}}+\frac{\partial v^{\prime}}{\partial y^{*}}\right)=0
$$

Dropping the "*" notation, used to denote the non-dimensionalized quantities, the equations in perturbation quantities for both non-uniform and uniform flow cases are written in the following way.

\section{Non-Uniform Mean Flow Equations}

$$
\begin{gathered}
\frac{\partial u^{\prime}}{\partial t}+\bar{u} \frac{\partial u^{\prime}}{\partial x^{*}}+\bar{v} \frac{\partial u^{\prime}}{\partial y^{*}}+\frac{1}{\bar{\rho}} \frac{\partial p^{\prime}}{\partial x}=-u^{\prime} \frac{\partial \bar{u}}{\partial x}-v^{\prime} \frac{\partial \bar{u}}{\partial y}+\frac{\partial \bar{p}}{\partial x} \frac{\rho^{\prime}}{\rho^{2}} \\
\frac{\partial v^{\prime}}{\partial t}+\bar{u} \frac{\partial v^{\prime}}{\partial x}+\bar{v} \frac{\partial v^{\prime}}{\partial y}+\frac{1}{\bar{\rho}} \frac{\partial p^{\prime}}{\partial y}=-u^{\prime} \frac{\partial \bar{v}}{\partial x}-v^{\prime} \frac{\partial \bar{v}}{\partial y}+\frac{\partial \bar{p}}{\partial y} \frac{\rho^{\prime}}{\bar{\rho}^{2}} \\
\frac{\partial p^{\prime}}{\partial t}+\bar{u} \frac{\partial p^{\prime}}{\partial x}+\bar{v} \frac{\partial p^{\prime}}{\partial y}+\gamma \bar{p}\left(\frac{\partial u^{\prime}}{\partial x}+\frac{\partial v^{\prime}}{\partial y}\right)=-u^{\prime} \frac{\partial \bar{p}}{\partial x}-v^{\prime} \frac{\partial \bar{p}}{\partial y}-\gamma\left(\frac{\partial \bar{u}}{\partial x}+\frac{\partial \bar{v}}{\partial y}\right) p^{\prime} \\
\frac{\partial \rho^{\prime}}{\partial t}+\bar{u} \frac{\partial \rho^{\prime}}{\partial x}+\bar{v} \frac{\partial \rho^{\prime}}{\partial y}+\bar{\rho}\left(\frac{\partial u^{\prime}}{\partial x}+\frac{\partial v^{\prime}}{\partial y}\right)=-\rho^{\prime}\left(\frac{\partial \bar{u}}{\partial x}+\frac{\partial \bar{v}}{\partial y}\right)-u^{\prime} \frac{\partial \bar{\rho}}{\partial x}-v^{\prime} \frac{\partial \bar{\rho}}{\partial y}
\end{gathered}
$$




\section{APPENDIX (continued)}

\section{Uniform Mean Flow Equations}

$$
\begin{gathered}
\frac{\partial u^{\prime}}{\partial t}+M_{x} \frac{\partial u^{\prime}}{\partial x}+M_{y} \frac{\partial u^{\prime}}{\partial y}+\frac{\partial p^{\prime}}{\partial x}=0 \\
\frac{\partial v^{\prime}}{\partial t}+M_{x} \frac{\partial v^{\prime}}{\partial x}+M_{y} \frac{\partial v^{\prime}}{\partial y}+\frac{\partial p^{\prime}}{\partial y}=0 \\
\frac{\partial p^{\prime}}{\partial t}+M_{x} \frac{\partial p^{\prime}}{\partial x}+M_{y} \frac{\partial p^{\prime}}{\partial y}+\left(\frac{\partial u^{\prime}}{\partial x}+\frac{\partial v^{\prime}}{\partial y}\right)=0 \\
\frac{\partial \rho^{\prime}}{\partial t}+M_{x} \frac{\partial \rho^{\prime}}{\partial x}+M_{y} \frac{\partial \rho^{\prime}}{\partial y}+\left(\frac{\partial u^{\prime}}{\partial x}+\frac{\partial v^{\prime}}{\partial y}\right)=0
\end{gathered}
$$

